\author{
Universidade de São Paulo \\ Instituto de Física de São Carlos
}

Thiago Schiavo Mosqueiro

\title{
Transições Ópticas em Heteroestruturas Semicondutoras Zincblende com duas Sub-bandas
}

São Carlos 



\section{Thiago Schiavo Mosqueiro}

\section{Transições Ópticas em Heteroestruturas Semicondutoras Zincblende com duas Sub-bandas}

Dissertação apresentada ao Programa de PósGraduação em Física do Instituto de Física de São Carlos da Universidade de São Paulo, para obtenção do título de mestre em Ciências.

Área de Concentração: Física Básica

Orientador: Prof. Dr. Esmerindo Bernardes

Versão Corrigida

(versão original disponível na Unidade que aloja o Programa)

São Carlos 
AUTORIZO A REPRODUÇÃO E DIVULGAÇÃO TOTAL OU PARCIAL DESTE TRABALHO, POR QUALQUER MEIO CONVENCIONAL OU ELETRÔNICO, PARA FINS DE ESTUDO E PESQUISA, DESDE QUE CITADA A FONTE.

Ficha catalográfica elaborada pelo Serviço de Biblioteca e Informação IFSC/USP

Mosqueiro, Thiago Schiavo

Transições ópticas em heteroestruturas semicondutoras Zincblende com duas sub-bandas. / Thiago Schiavo Mosqueiro; orientador Esmerindo Bernardes - versão corrigida -- São Carlos, 2011.

$160 \mathrm{p}$.

Dissertação (Mestrado - Programa de Pós-Graduação em Física Área de concentração: Física Básica - Instituto de Física de São Carlos da Universidade de São Paulo.

1. Spintrônica. 2. Poços quânticos. 3. Acoplamento spin-órbita. 4. Eletrodinâmica quântica. I. Título. 


\section{AGRADECIMENTOS}

Gostaria de deixar registrado agradecimentos a diversas pessoas. Sem algumas, não haveria dissertação: meus pais, Renato e Vera, minha companheira de longa data, Jaqueline, e meu colega de discussões bem abrangentes, Brenno. Ainda com eterna gratidão, agradeço imensamente ao meu orienador, prof. Esmerindo Bernardes, a quem devo muito de meu amadurecimento e com quem tive a chance de trabalhar e criar ciência.

Garanto, hoje, que sem sociedade não há ciência. E minha sociedade é uma rica variedade de pessoas que me ajudaram em diversos níveis de meu aprendizado: prof. Francisco Alcaraz, prof. Francisco Guimarães, prof. Leonardo Maia, prof. Guilherme Sipahi, prof. Tito José Bonagamba, Claudinei, Cora, Daniele Jacinto, João Bunoro, Jorge Kondo, Gabriel Luchini, Guilherme Prata, Julian Jaimes (e Adriana!), Márcio Cobo, Mariana Odashima, Oigres Daniel, Paulo Eduardo de Faria Jr., Paulo Hisao, Paweł Klimas, Rafael Guolo, Rafael Viegas, Tiago Batalhão, Tiago Campos e William Trevizan. O pessoal do LeGauss, em principal Rodrigo e Tiago Fonseca.

A meus companheiros de grupo, agradeço por valiosas discussões: Felipe, Filipi, Gerson, Marco, Poliana, Jyong Fu e ao prof. Egues. Adiciono aqui stambém as secretárias Cristina, Mariana e Yvone como integrantes de nosso grupo. Agradeço com especial gratidão a Rafael Calsaverini por discussões que me iniciaram neste estudo. Aos professores Marcel Novaes e louri Poussep - integrantes de minha banca - e ao estudante Diego, obrigado por críticas, sugestões e correções.

Ao pessoal da Biblioteca do IFSC e da secretaria da Pós, que sempre me espantam com sua dedicação, também deixo marcados meus agradecimentos: Ana Mara, Maria Cristina, Neusa, Ricardo, Sílvio e Vitor.

Além de minha sociedade local, recebi durante meus anos de dedicação apoio de meu irmão, Kim, de Mônica Lapini (minha madrinha de crisma), de Tárcia Garcia Leal (ex professora), de Margit (ex professora), de Marcela e Rafael (meus afilhados de casamento), do Amadeu (por alimentar minhas ideias em horas avançadas da noite), Angélica e Jorge Saba 
(tia e marido). Além destes, destaco também colegas mais distantes espacialmente: Raphael, Hélio, Getúlio Gaeta.

Não poderiam faltar agradecimentos à sociedade que tornou possível a realização de meus projetos e trabalhos: a comunidade de software livre. Esta dissertação e o trabalho em si foram desenvolvidos com Linux (Kernel 2.6.34.7-61.fc13.x86_64), Fedora 13 - Goddard -, texlive, kile, TeXmacs, Tikz/PGF, gnuplot 4.2 (6), gfortran 4.4 .5 e python 2.6.4.

Finalizo agradecendo minha futura sociedade, os leitores deste trabalho. 
Everyone believes in the (normal) law of errors: the mathematicians, because they think it is an experimental fact; and the experimenters, because they suppose it is a theorem of mathematics.

Gabriel Lippmann, um fotógrafo e ganhador do Prêmio Nobel de Física, em conversa com Henri Poincaré, disponível na obra Calcul des Probabilités (1986), p. 171. 



\section{RESUMO}

MOSQUEIRO, T. S. Transições ópticas em heteroestruturas semicondutoras Zincblende com duas sub-bandas. 2011. 160 p. Dissertação de Mestrado - Instituto de Física de São Carlos, Universidade de São Paulo, São Carlos, 2011.

Apresento neste trabalho uma derivação alternativa da hamiltoniana efetiva para um elétron na banda de condução de uma heteroestrutura semicondutora de rede Zincblende. Partindo do modelo de Kane $8 \times 8$ e da aproximação das funções envelope, esta hamiltoniana efetiva foi obtida com a linearização dos denominadores (dependentes das autoenergias) presentes na equação para a banda de condução, sob a hipótese de que o gap de energia seja muito maior que todas as demais diferenças de energia envolvidas (verdade para a maioria das estruturas Zincblende). A partir de um procedimento introduzido previamente ${ }^{1,3}$, desenvolvi um procedimento mais geral que implementa sistematicamente esta linearização até segunda ordem no inverso do gap de energia e que corrige a normalização do spinor da banda de condução usando as bandas de valência. Este procedimento é idêntico à expansão em série de potência no inverso da velocidade da luz utilizada para se obter aproximações relativísticas da equação de Dirac. Uma vantagem deste procedimento é a arbitrariedade na forma dos potenciais, o que implica na validade da hamiltoniana resultante para poços, fios e pontos quânticos. Evidencio também as consequências de cada termo desta hamiltoniana efetiva para os autoestados eletrônicos em poços retangulares, incluindo termos independentes de spin inéditos (Darwin e interação momento linear-campo elétrico). Estes resultados estão de acordo com os estudos anteriores ${ }^{4}$. A fim de estudar transições ópticas dentro da banda de condução, mostro que o acoplamento mínimo pode ser realizado diretamente na hamiltoniana de Kane se os campos externos variam tão lentamente quanto as funções envelope. Repetindo a linearização dos denominadores de energia, derivo uma hamiltoniana efetiva para a banda de condução com acoplamentos elétron-fótons. Um destes acoplamentos, induzido exclusivamente pela banda de valência, origina transições mediadas pelo spin eletrônico. Estas transições assistidas por 
spin possibilitam mudanças, opticamente induzidas, na orientação do spin eletrônico, um fato que talvez possa ser útil na construção de dispositivos spintrônicos. Por fim, as taxas de transição deste acoplamento apresentam saturação e linhas de máximos e mínimos no espaço recíproco. Espero que estas acoplamentos ópticos possam auxiliar na observação dos efeitos dos acoplamentos spin-órbita intra (Rashba) e intersubbandas.

PAlavras-Chave: Spintrônica. Poços quânticos. Acoplamento spin-órbita. Eletrodinâmica quântica. 


\section{ABSTRACT}

MOSQUEIRO, T. S. Optical transitions in Zincblende semiconductors heterostructures with two sub-bands. 2011. 160 p. Dissertação de Mestrado - Instituto de Física de São Carlos, Universidade de São Paulo, São Carlos, 2011.

In this work, I present an alternative derivation of the conduction band effective hamiltonian for Zincblende semiconductor heterostructures. Starting from the $8 \times 8$ Kane model and envelope function approximation, this effective hamiltonian was obtained by means of a linearization in the eigenenergy-dependent denominators present the conduction band equation, under the hypothesis that the energy gap is bigger than any other energy difference in the system (true for mostly every Zincblende semiconductor - bulk or heterostructure). Based on a previous procedure $^{1,3}$, I have developed a more general procedure that implements sistematicaly (i) this linearization and (ii) renormalizes the conduction band spinor using the valence bands, both (i) and (ii) up to second order in the inverse of the energy gap. This procedure is identical to the expansion in power series of the inverse of the light speed used to derive non-relativistic approximations of the Dirac equation. One advantage of this procedure is the generality of the potentials adopted in our derivation: the same results hold for quantum wells, wires and dots. I show the consequences of each term of this hamiltonian for the electron eigenstates in retangular wells, including novel spin-independent terms (Darwin and linear momentum-electric field couplings). These resulties agree with previous works ${ }^{4}$. In order to study conduction band optical transitions, I show that the minimal substitution can be performed directly in the Kane hamiltonian if the external fields vary slowly (at least, as slowly as the envelope functions). Repeating the linearization of the energy denominators, I derive a new effective hamiltonian, up to second order in the inverse of the energy gap, with electron-photons couplings. One of these couplings, induced exclusively by the valence bands, gives rise to optical transitions mediated by the electron spin. This "spin-assisted" coupling enable optically-induced spin flipps in conduction subband transitions, which can be 
useful in the construction of spintronic devices. Finaly, the spin-assisted transitions rates show saturation and lines of maxima and minima in the reciprocal lattice. I hope that these optical couplings can be of any help in the observation of interesting effects induced by the intra and intersubband spin-orbit coupling.

KEYWORDS: Spintronics. Quantum well. Spin-orbit coupling. Quantum electrodynamics. 


\section{LISTA DE FIGURAS}

1.1 Esquema de um poço quântico. . . . . . . . . . . . . . . . . . . . . p. p. 22

1.2 Foto de um ponto quântico. . . . . . . . . . . . . . . . . . . . . . . . p. 23

1.3 Quebra de degenerescência de spin por Rashba . . . . . . . . . . . p. 26

1.4 Transistor de Efeito de Campo de Spin . . . . . . . . . . . . . . . . . . . p. 27

2.1 Simetria das funções de base para zincblende. . . . . . . . . . . . . . . . . p.37

2.2 Estrutura de bandas de materiais com rede zincblende. . . . . . . . . . . p p.39

2.3 Ilustração da quebra de simetria de translação e funções envelope. . . . . . p.40

2.4 Perfil dos potenciais estruturais do poço quântico. . . . . . . . . . . . p. p6

3.1 Autoconsistência nos cálculos numéricos . . . . . . . . . . . . p. 66

3.2 Soluções autoconsistentes do poço quântico . . . . . . . . . . . . . . . . p. p8

3.3 Detalhes sobre o acoplamento intersub-bandas. . . . . . . . . . . . . p.71

3.4 Estimativas do acoplamento momento-campo elétrico . . . . . . . . . . p.73

3.5 Estimativas para o termo de Darwin . . . . . . . . . . . . . . . p.77

3.6 Quebra de degenerescência via quebra de simetria. . . . . . . . . . p. 80

3.7 Campos magnéticos internos . . . . . . . . . . . . . . . . . . p. 83

3.8 As linhas de campo do campo magnético interno . . . . . . . . . . p. 86

4.1 Polarização em plano e perpendicular . . . . . . . . . . . . . . . p.96

4.2 Ilustração da aproximação de dipolo elétrico. . . . . . . . . . . . . . . . p.97

4.3 Ilustração do comportamento assintótico das taxas de transições. . . . . . p.117

5.1 Frequências características dos poços quânticos com que trabalhamos. . . . p. 122 
5.2 Elementos de matriz do acoplamento assistido por spin. . . . . . . . . p. 128

5.3 Particularidades dos elementos de matriz do acoplamento assistido por spin. p. 129

5.4 Acoplamento assistido por spin variando parâmetros . . . . . . . . . . . p. 130 


\section{LISTA DE TABELAS}

2.1 Funções de bloch na base de momento angular. . . . . . . . . . . . . p p.38

4.1 Comparação dos acoplamentos elétron-fóton. . . . . . . . . . . . p. 95 



\section{LISTA DE SÍMBOLOS}

$:=\quad$ Sinal de definição

c.c. Complexo conjugado

$\otimes \quad$ Produto diádico, direto ou tensorial

$\mathcal{P} \quad$ Parâmetro de Kane

$E_{g} \quad G a p$ do material central do poço quântico

$\Delta_{g} \quad$ Split-off do material central do poço quântico

$\gamma_{1} \quad \frac{2}{E_{g}}+\frac{1}{E_{g}+\Delta_{g}}$

$\gamma_{2} \quad \frac{2}{E_{g}^{2}}+\frac{1}{\left(E_{g}+\Delta_{g}\right)^{2}}$

$\eta_{H} \quad \frac{1}{E_{g}^{2}}-\frac{1}{\left(E_{g}+\Delta_{g}\right)^{2}}$.

$\eta_{e} \quad \frac{\delta_{8}}{E_{g}^{2}}-\frac{\delta_{7}}{\left(E_{g}+\Delta_{g}\right)^{2}}$

$\gamma_{2}^{\prime} \quad \frac{2 \delta_{8}}{E_{g}^{2}}+\frac{\delta_{7}}{\left(E_{g}+\Delta_{g}\right)^{2}}$ 



\section{SUMÁRIO}

1 Introdução $\quad$ p. 21

1.1 Semicondutores e heteroestruturas . . . . . . . . . . . . . p. 21

1.2 Acoplamento spin-órbita e poços quânticos . . . . . . . . . . . . . . . . p. 24

1.2.1 Transistor de Efeito de Campo de Spin . . . . . . . . . . . . . . p. 26

1.3 Dispositivos optoeletrônicos com poços quânticos . . . . . . . . . . . . . . p. 28

1.4 Objetivo e divisão dos tópicos . . . . . . . . . . . . . . . . . . . . . . . p. 29

1.5 Comentários sobre notação . . . . . . . . . . . . . . . . . . p. 30

2 Hamiltoniana Efetiva para Banda de Condução p. 33

2.1 Método k p . . . . . . . . . . . . . . . . . p. . . . . . . . . .

2.2 Modelo de Kane-Bulk . . . . . . . . . . . . . . . . . . p. 36

2.3 Quebra de simetria e as funções envelope . . . . . . . . . . . . . . . . . p.38

2.3.1 Aproximação das funções envelope . . . . . . . . . . . . . . . . . p. p. 41

2.3.2 Equação para as funções envelope . . . . . . . . . . . . . . p. p3

2.4 Hamiltoniana de Kane para heteroestruturas . . . . . . . . . . . . . p. 44

2.5 Álgebra das matrizes $T_{j} \ldots \ldots \ldots \ldots \ldots$. . . . . . . . . . . . . . . . . . . . . . .

2.5.1 Desenvolvimento dos operadores escalares . . . . . . . . . p. 49

2.5.2 Aplicação com ponto quântico . . . . . . . . . . . . . . . . . p. 49

$2.6 E_{g}$ como maior energia envolvida . . . . . . . . . . . . . p. 51

2.7 Equação efetiva para banda de condução . . . . . . . . . . . . . . . p.52 
2.7.1 Normalização apropriada: interpretação probabilística . . . . . . p. 54

2.7.2 Hamiltoniana efetiva . . . . . . . . . . . . . p. 56

2.7.3 Finalizando: representação em $\langle r| \ldots \ldots$. . . . . . . . . . . p. 58

2.8 Objeção de Ando . . . . . . . . . . . . . . . . . . . . . . . . . p.60

2.9 Revisão . . . . . . . . . . . . . . . . . . . . . . . . p. 61

3 Solução para a Banda de Condução $\quad$ p. 63

3.1 Soluções semianalíticas para a equação efetiva . . . . . . . . . . . p. 63

3.1 .1 Sobre $H_{\mathcal{N}}$ e separação de variáveis . . . . . . . . . . . . . . p. 64

3.1 .2 Cálculos numéricos . . . . . . . . . . . . . . . p. 66

3.1 .3 Adição de barreiras . . . . . . . . . . . . . . . . p. 67

3.2 Diagonalização do acoplamento spin-órbita e representação matricial . . p.68

3.3 Acoplamentos intra- e intersub-bandas . . . . . . . . . . . . p. 70

3.4 Poço perfeitamente retangular . . . . . . . . . . . . . . p. 71

3.4.1 Acoplamento momento-campo elétrico . . . . . . . . . . . p. p. 72

3.4.2 Correção estrutural análoga à de Darwin . . . . . . . . . . . . p. 75

3.4.3 Correção do potencial eletrônico análoga à de Darwin . . . . . . . p. p 77

3.5 Relação de dispersão . . . . . . . . . . . . . . . . . . . . . . . p. 78

3.5 .1 Caso simétrico . . . . . . . . . . . . . . . . . . . . . p. 78

3.5.2 Caso geral . . . . . . . . . . . . . . . . . . . . . . . .

3.6 A banda de condução . . . . . . . . . . . . . . . . . . p. 80

3.6 .1 Caso simétrico . . . . . . . . . . . . . . . . . p. 80

3.6 .2 Caso geral ...................... . . . . . 81

3.7 Caso com uma sub-banda . . . . . . . . . . . . . . . . . . . . . p. 82

3.8 Hamiltoniana $4 \times 4$ e os campos magnéticos internos . . . . . . . . p. 83

3.9 Revisão . . . . . . . . . . . . . . . . . . . . . . . . . . . . . . p. 87 
4 Modelagem da Interação Elétron-Fóton $\quad$ p. 89

4.1 Hamiltoniana com interação elétron-fóton . . . . . . . . . . . . . . p.89

4.1.1 Acoplamento elétron-fóton: uma visão de $\mathbf{k} \cdot \mathbf{p} \ldots$. . . . . . . . p. 90

4.2 Quantização do campo eletromagnético . . . . . . . . . . . . . p.91

4.2.1 Os diferentes acoplamentos e ordens de grandezas . . . . . . . . . p. 94

4.2.2 Base com número de fótons . . . . . . . . . . . . . . . p. 95

4.3 Aproximação de dipolo elétrico f . . . . . . . . . . . . . . . . . . . . p.97

4.4 Aproximação das Funções Envelope com Campo de Radiação .......98

4.5 Equação efetiva para banda de condução com campo de radiação . . . . . . p. 100

4.5.1 Ação dos operadores com campo de radiação . . . . . . . . . . . . p. 103

4.5.2 Termos quadráticos no potencial vetor . . . . . . . . . . . . p. 104

4.5 .3 Normalização . . . . . . . . . . . . . . . . . . . . . . . . p. 105

4.6 Interação elétrons-fóton em primeira ordem . . . . . . . . . . . . . p.106

4.7 Interação elétrons-fóton até segunda ordem . . . . . . . . . . . . . p. 107

4.8 Interação dependente do tempo . . . . . . . . . . . . . . . . . . p. 111

4.8.1 Tratamento específico para as transições ópticas . . . . . . . . . . . p. 114

4.8.2 Diagramas de Feynman: interações de um fóton . . . . . . . . . . p. 115

4.8.3 Aproximação de dipolo elétrico e transições indiretas . . . . . . . . p. 116

4.9 Transições Ópticas usando Regra de Ouro . . . . . . . . . . . . . . . . . p.117

4.9 .1 Matriz óptica . . . . . . . . . . . . . . . . . . . . p. 118

4.10 Revisão . . . . . . . . . . . . . . . . . . . . . . . . . p. 120

5 Taxas de Transições $\quad$ p. 121

5.1 Frequências características . . . . . . . . . . . . . . . . . . . . . . p. 121

5.2 Elementos da matriz óptica f . . . . . . . . . . . . . . . . . . p. 122

5.2 .1 Acoplamento usual . . . . . . . . . . . . . . . . . . . . . p. 123

5.2.1.1 Caso simétrico . . . . . . . . . . . . . . . . p. 125 
5.2.2 Acoplamento radiação-momento-campo elétrico . . . . . . . . . . p. 125

5.2.3 Acoplamento assistido por spin . . . . . . . . . . . . . . . p. 126

5.2.4 Aplicação de gates e largura do poço . . . . . . . . . . . . . . . . p. 130

5.3 Revisão . . . . . . . . . . . . . . . . . . . . . . . . . . . . . . . p. 132

6 Conclusões do Trabalho p. 135

REFERÊNCIAS p. 139

APÊNDICE A - Cálculos auxiliares com matrizes T $\quad$ p. 147

A.1 Desenvolvimento de algumas expressões . . . . . . . . . . . . . . . . . . p. 147

A.2 Expressão para a massa efetiva . . . . . . . . . . . . . . . . . . . p. 148

A.3 Hermitianicidade de uma expansão de Taylor . . . . . . . . . . . . . . . . . p. 148

APÊNDICE B - Expansão não relativística da equação de Dirac p. 151

B.1 Equação efetiva de partículas . . . . . . . . . . . . . . . . . . . . p. 151

B.2 Correção da normalização e equação efetiva para partículas . . . . . . . . p. 152

APÊNDICE C - Comparação com Winkler p. 155

APÊNDICE D - Detalhes sobre a diagonalização $\quad$ p. 157

D.1 Hamiltoniana efetiva e a base de estados . . . . . . . . . . . . . . p. 157

D.2 Revisitando Álgebra Linear . . . . . . . . . . . . . . . . . . . . . p. 159

D.3 Solução para a banda de condução . . . . . . . . . . . . . . . . . . . . . p. 160 


\section{CAPÍTULO 1}

\section{Introdução}

Dio, can you hear me? I am lost and so alone

I am asking for your guidance, could you come down from your throne?

Jables, em Tenacious D - Kickapoo, 2007.

Neste capítulo, apresento ao leitor o ambiente deste trabalho. Veremos um pouco sobre o acoplamento spin-órbita, e sua origem na equação de Dirac. Também comento brevemente a presença deste acopalmento em técnicas atuais de desenvolvimento de dispostiviso eletrônicos. Este último revela uma área nova e crescente, a spintrônica. Por fim, podemos encarar este capítulo como uma revisão bibliográfica.

\subsection{Semicondutores e heteroestruturas}

Hoje vivemos a fase da informação: geração, armazenamento, recuperação e tratamento; a atenção de diversos projetos voltam-se ao tratamento da informação ou da otimização dos processos relacionados. Tão interessante quanto isto é o fato de a maioria dos produtos desta sociedade, amante da informação, girar em redor de um único semicondutor razoavelmente abundante: Silício (Si). Não é a toa que alguns antropólogos e sociólogos definem esta época histórica como a Idade do Silício. A manipulação e o armazenamento de informação, a baixo nível (i.e., de hardware), baseiam-se em transistores. Este componente eletrônico é responsável pela grande revolução da eletrônica da década de 1960, além de levar ao prêmio Nobel os físicos William Bradford Shockley, John Bardeen e Walter Houser Brattain. Os primeiros transistores foram fabricados nos anos 50 com Germânio (Ge). No entanto, logo notaram-se diferenças entre Ge e Si que determinaram os rumos da tecnologia moderna: o gap do Si é o dobro do gap do Ge); a corrente reversa do Si é consequentemente menor que a do $\mathrm{Ge}$; a temperatura máxima de operação do Si $\left(150^{\circ} \mathrm{C}\right)$ é maior que a do Ge $\left(100^{\circ} \mathrm{C}\right)$. Além 


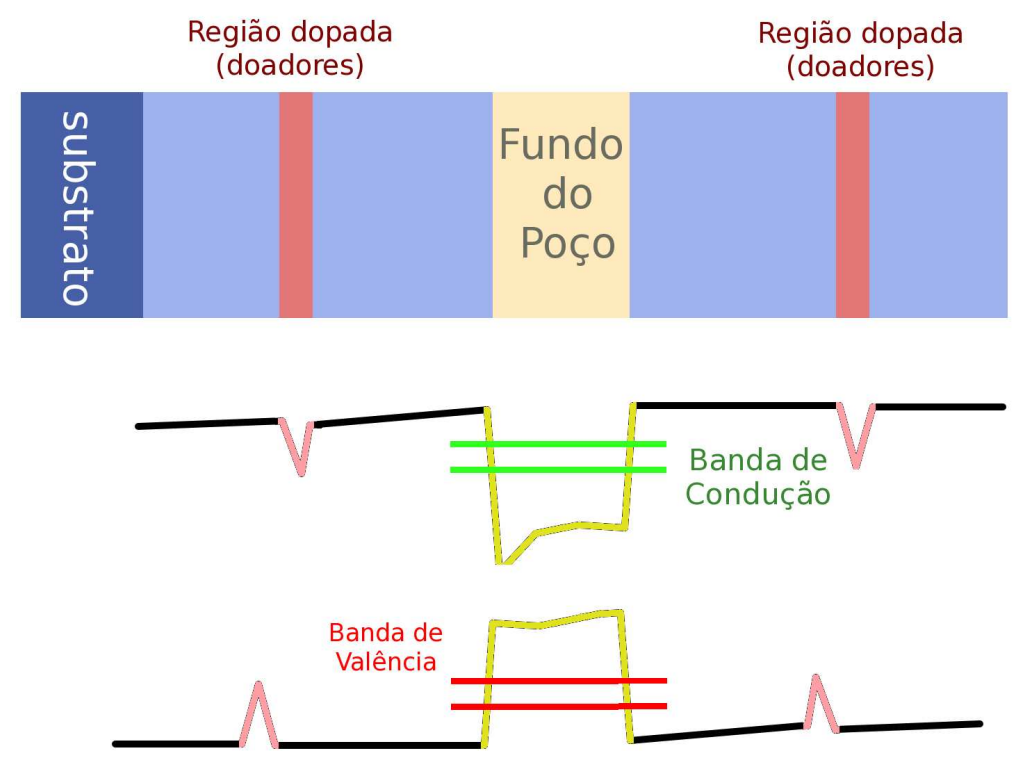

Figura 1.1 - Um poço quântico é a superposição sequencial de três estruturas semicondutoras, sendo que a do meio tem o menor gap. A diferença de gaps pode ser encarado, pictoricamente, como um perfil de potencial de poço quântico. Na figura, o poço é de tipo $I$, i.e., o potencial para as bandas de valência é uma barreira (oposto do potencial da banda de condução).

disso, o Si é oxidável termicamente, o que constitui a base dos processos de fabricação dos circuitos integrados ${ }^{6}$. Mais recentemente, novos materiais, e.g., GaAs, marcam presença em dispositivos mais modernos embora em menor escala principalmente por questões financeiras.

Heteroestruturas são junções de materiais com características diferentes. Em especial, heteroestruturas semicondutoras são exploradas com mais entusiasmo por já se dominarem as técnicas de crescimento de junções formadas por semicondutores. A heteroestrutura mais simples é o poço quântico: uma sequência de três semicondutores, conectados por interfaces paralelamente alinhadas, em que o material de menor gap fica no centro. Efetivamente o potencial experimentado pelos elétrons na banda de condução tem um perfil de poço, como vemos na figura 1.1. Portanto essa junção confina esses elétrons de condução da região do material central, formando níveis de estados ligados. Assim como um poço quântico que estudamos nos livros texto ${ }^{7}$, o número de estados ligados depende, por exemplo, da largura do poço.

Como nas direções paralelas ao plano das junções não há quebra de simetria de translação, tudo se passa como em bulk*. Portanto, o poço quântico confina um gás de elétrons bidimensi-

\footnotetext{
*Palavra amplamente utilizada para referir estruturas puras (do inglês, significa "volume").
} 


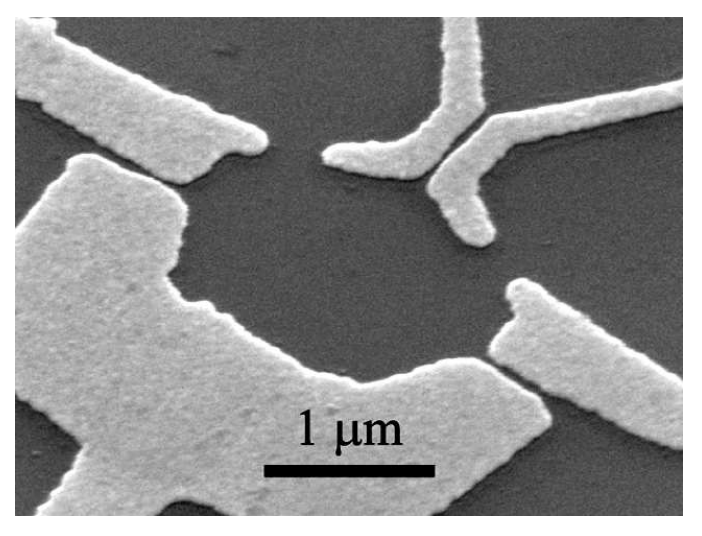

Figura 1.2 - Foto, por microscopia, de um ponto quântico. Foto retirada do site do grupo do prof. Schröenberger (Experimental Mesoscopic Physics), disponível em: $<$ http://pages . unibas.ch/phys-meso/index-M.html>. Acesso em: Novembro de 2010.

onal - do inglês, two-dimensional electron gas (2DEG) - em sua região central. Analogamente, exsitem também o fio quântico (do inglês, quantum wire), que confina um gás unidimensional de elétrons, e o ponto quântico (do inglês, quantum dot), que confina elétrons em todas as direções. Cada uma destas heteroestruturas são estudadas exaustivamentes por suas propriedades interessantes e comerciais. Por exemplo, em 1998, foi proposto o armazenamento de qubits $^{\dagger}$ em pontos quânticos ${ }^{8}$. Além disso, pontos quânticos são utilizados para gerar luz com frequência muito bem controlada ${ }^{10}$ (e dispositivos optopeletrônicos em geral ${ }^{9}$ ). Na figura 1.2 podemos ver uma foto de um ponto quântico.

Como as técnicas de crescimento destas estruturas vêm se aprimorando rapidamente, surgem novas heteroestruturas cada vez mais sofisticadas e puras (sem defeitos). Há, por exemplo, as super-redes, que basicamente são múltiplos poços quânticos unidos sequencialmente. Uma novidade que surgiu nestes últimos anos é o politipismo: super-redes formadas por estruturas com simetrias diferentes, tal como unir zincblende com wurtzita ${ }^{11}$ - duas estruturas separadas por uma transição de fase em muitos casos. Cada uma destas montagens têm vantagens comerciais interessantes, tais como desenvolvimento de materiais optoeletrônicos.

Mais recentemente, têm-se proposto modelos inovadores para transistores baseados no spin eletrônico, dando origem a um dos grandes desafios atuais: a construção de um computador quântico. O computador quântico não deve apenas armazenar confiavelmente dados em qubits, mas também deve implementar portas lógicas sobre as quais se constroem os algoritmos e as tarefas que todo computador deve ser capaz de realizar. Atualmente há diversas propostas e

\footnotetext{
${ }^{\dagger}$ Analogamente aos bits dos computadores atuais, qubits são as unidades fundamentais para armazenar in-
} formação em um (futuro) computador quântico. 
discussões sobre a forma de manipular os qubits (e.g., controle óptico do spin eletrônico ${ }^{12}$ ) e sobre o uso de materiais emergentes (e.g., grafeno ${ }^{13}$ ). Costuma-se chamar de spintrônica ${ }^{\ddagger}$ a área que estuda e tenta implementar estes modelos, ou seja, a ciência do controle do transporte de elétrons com spins bem determinados. A spintrônica ganhou grande notoriedade a partir de experimentos de transporte eletrônico dependente de spin em dispositivos de estado sólido nos anos 80: observação da injeção de elétrons com spin polarizados em metais ferromagnéticos ${ }^{14}$ e a descoberta da magnetoresistência gigante, reportada nos papers de Baibich et al ${ }^{15} \mathrm{e}$ de Binasch et $a^{16}$. Atualmente, a spintrônica move grande parcela da pesquisa básica em semicondutores ${ }^{17}$ por ser um excelente laboratório para novos efeitos físicos.

\subsection{Acoplamento spin-órbita e poços quânticos}

A spintrônica baseia-se no efeito do acoplamento entre o spin eletrônico e seus estados orbitais. Neste trabalho, estamos interessados em estudar junções semicondutoras, focados inteiramente nos elétrons da banda de condução que são aprisionados no poço. Há dois acoplamentos spin-órbita bem conhecidos na literatura para tais elétrons: de Rashba, existente quando há assimetria no perfil do potencial do poço ${ }^{18}$ - quebrando a degenerescência de spin na banda de condução ${ }^{19}$-, e o de Dresselhauss ${ }^{20}$, existente quando não há simetria de inversão na rede cristalina ${ }^{21}$. Vamos a seguir discutir brevemente sobre como surgem estes acoplamentos.

A equação de Dirac, que descreve elétrons em regimes relativísticos, trabalha com quatro componentes - duas a mais que a equação de Schrödinger (com spin) -, que podem ser descritos por quadrivetores ou spinores ${ }^{22}$. No entanto, é possível desvincular estas quatro componentes, separando o quadrivetor em dois spinores de duas componentes. Por meio de expansões em potências de $1 / c$, é possível deduzir equações efetivas para cada um destes spinores. Pode-se mostrar que um dos spinores anula-se em primeira ordem e é muito pequeno em segunda ordem. O outro spinor, no entanto, obedece à equação de Pauli em primeira ordem e, em segunda, ganha três "correções relativísticas". À medida com que expandimos em sucessivas ordens de $1 / c$, obtemos equações análogas à de Schrödinger, mas com "correções relativísticas" 22 (Bjorken e Drell têm uma referência mais atual sobre o assunto $\left.^{23}\right)$. O acoplamento spin-órbita é uma dessas correções em ordem de $1 / c^{2}$. Há duas outras correções relativísticas nesta ordem: o conhecido termo de Darwin e uma correção para a massa eletrônica (termo proporcional a $P^{4}$ ). $\mathrm{O}$ acoplamento spin-órbita pode ser escrito na

\footnotetext{
$\ddagger$ Um tipo de neologismo gerado a partir de eletrônica do transporte de spin. Encara-se como uma nova eletrônica, tal como a fotônica.
} 
forma

$$
H_{s o}=\frac{\hbar}{4 m^{2} c^{2}} \boldsymbol{p} \cdot \boldsymbol{\sigma} \times \nabla V(\boldsymbol{r}),
$$

em que $\boldsymbol{\sigma}=\sigma_{x} \hat{x}+\sigma_{y} \hat{y}+\sigma_{z} \hat{z}$ e $\sigma_{j}$ são as matrizes de Pauli. Sempre que o potencial for central, i.e., $V(\boldsymbol{r})=V(r)$ (simetria esférica), nota-se que

$$
H_{s o}=\frac{1}{2 m^{2} c^{2}} \frac{1}{r} \frac{d V}{d r}(r) \boldsymbol{L} \cdot \boldsymbol{S}
$$

forma mais comumente discutida em referências de Mecânica Quântica não-relativística 7,24,25 e importante para a determinação do espectro de diversos átomos e moléculas.

Na determinação da estrutura de bandas de semicondutores (estruturas homogêneas, ou bulks, ou heteroestruturas), podemos esperar, da adição de um termo como da equação 1.2.1, ao menos um desdobramento das bandas (álgebra simplética). Em semicondutores com assimetria de inversão espacial, que é o caso de estruturas Zincblende ${ }^{26-28}$, pode-se mostrar que este desdobramento decorre do acoplamento de Dresselhaus, também conhecido como acoplamento de Assimetria de Inversão em Bulk (BIA, do inglês bulk inversion asymmetry).

Em poços quânticos, o acoplamento $B I A$ continua presente se os materiais constituintes da junção não tiverem simetria de inversão espacial. A aplicação de uma diferença de potencial nas extremidades do poço é um exemplo de como criar um outro tipo de assimetria: a de inversão estrutural. Vamos definir $\hat{z}$ como a direção perpendicular à interface das junções (eixo de crescimento). Rashba ${ }^{\S}$ (1960 apud Bychkov e Rashba, 1986 ${ }^{28}$ ) mostrou que esta quebra de simetria gera outro acoplamento spin-órbita, conhecido como acoplamento de Assimetria de Inversão Estrutural (SIA, do inglês structural inversion asymmetry) ou acoplamento de Rashba, que para a banda de condução pode ser escrito como

$$
H_{r}=\alpha \boldsymbol{\sigma} \cdot \hat{z} \times \boldsymbol{k}
$$

em que $\alpha$ é a constante de acoplamento de Rashba. Esta constante depende do material e dos campos externos (tanto elétrico, como magnético). Podemos ver diversas características deste acoplamento spin-órbita reunidas na figura 1.3. Na seção 2.7 deduzirei uma equação para a banda de condução que contem naturalmente o acoplamento de Rashba e $\alpha$ será determinado adequadamente.

Recentemente novos estudos, experimentais ${ }^{29-32}$ e teóricos ${ }^{1-3,33}$, dedicam-se aos efeitos spin-órbita quando o poço admite duas sub-bandas. A maioria destes estudos focam-se em

\footnotetext{
$\S$ Rashba, E. Properties of semiconductors with an extremum loop. 1. cyclotron and combinatorial resonance in a magnetic field perpendicular to the plane of the loop. Soviet Physics: solid state, v. 2, n. 6, p. 1109-1122, 1960.
} 
materiais com estrutura Zincblende, mas é notável um crescente interesse em Wurtzita ${ }^{34}$. Observou-se recentemente um acoplamento intersub-bandas (significa entre sub-bandas diferentes), de mesma origem que o acoplamento de Rashba, que também pode ser controlado pela aplicação de campos externos. O valor deste acoplamento tem a mesma ordem de grandeza que o de Rashba. No entanto, há diferenças interessantes, e.g., este acoplamento não se anula em estruturas simétricas ${ }^{1,3}$, gera efeito Hall quântico não nulo ${ }^{2,5}$ e apresenta uma ressonância em poços quânticos duplos ${ }^{4,35}$. Fabian et al escrevem em um tipo de survey ${ }^{17}$ uma seção sobre este acoplamento spin-órbita. Este é o acoplamento em que vamos nos focar ao longo desta dissertação. Além disso, este acoplamento dá origem ao efeito zitterbewegung que ainda está sendo explorado ${ }^{3}$. Movimentos oscilatórios similares vêm sendo alvo de grande interesse, não apenas em poços quânticos mas também em grafenos e nanotubos de carbono ${ }^{37-40}$.

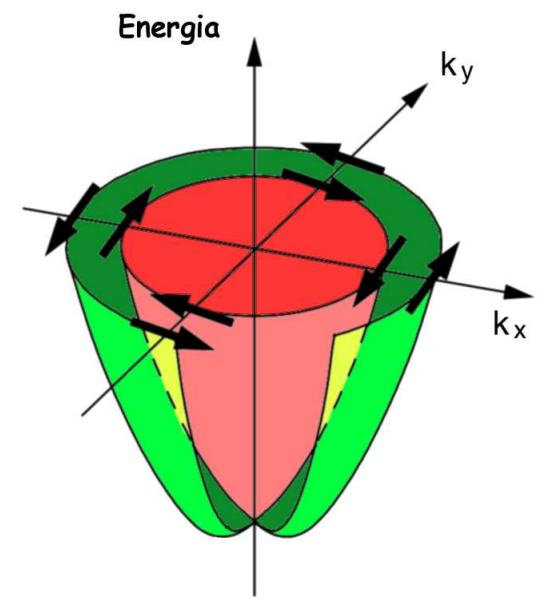

(a) Relação de disperção e polarização

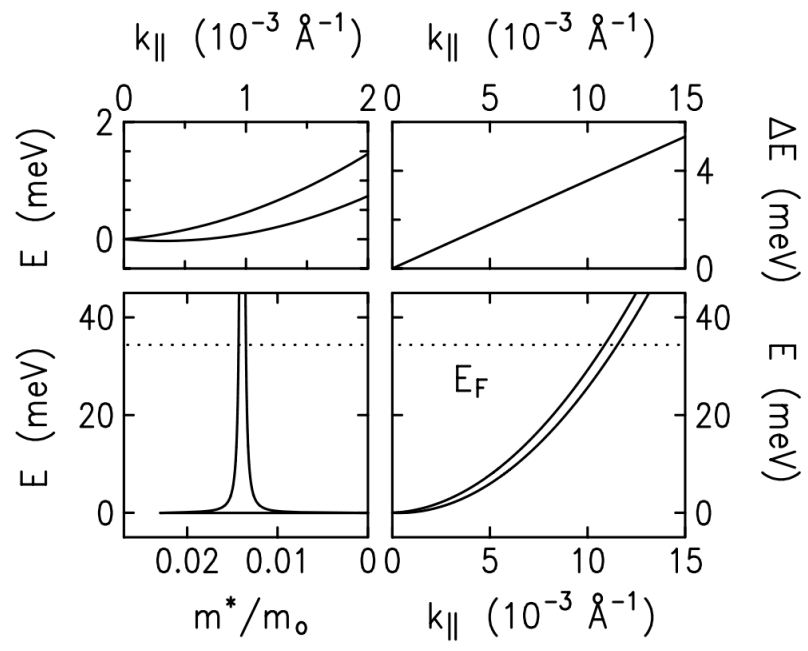

(b) Diversas características da relação de dispersão

Figura 1.3 - Assinaturas da hamiltoniana de Rashba em diversos sistemas: a relação de dispersão abre-se em duas superfícies quase parabólicas devido à quebra de degenerescência de spin (figura 3(a)); a diferença de energias é aproximadamente linear em $k_{\|}$e a massa efetiva de densidade de estados ${ }^{41}$ tem o comportamento mostrado nos gráficos à direita (figura 3(b), retirada da referência do Winkler ${ }^{41}$ ).

\subsubsection{Dispositivo spintrônico: Transistor de Efeito de Campo de Spin}

Um transistorll (usual) é um componente eletrônico - popularizado na década de 50 usado principalmente como amplificador e interruptor de sinais. A facilidade (e o baixo custo)

\footnotetext{
TDo alemão, "movimento oscilatório", assemelha-se a uma oscilação de Rabi ${ }^{36}$.

"Do inglês, Transfer Resistor - resistor de transferência.
} 


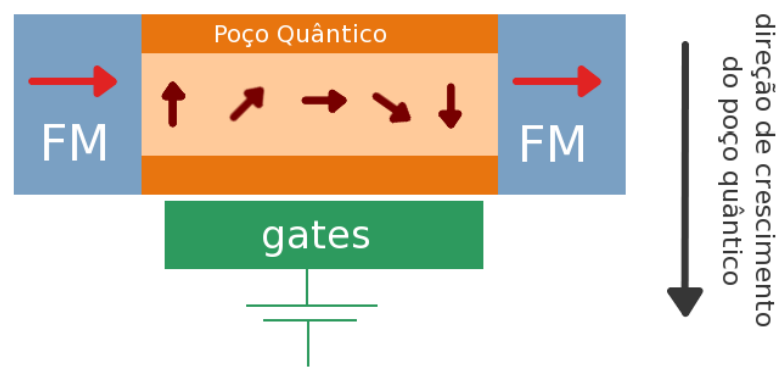

Figura 1.4 - Proposta de Datta e Das ${ }^{42}$ para o Spin-FET. A idéia primordial é controlar o estado final do spin de elétrons injetados dos contatos ferromagnéticos (denotados por FM). Recentemente foi implementada com sucesso ${ }^{43}$.

de produzir transistores se somam à engenhosa forma como conseguimos manipulá-los e criar dispositivos complexos para executar tarefas diversas. Atualmente, não há circuitos integrados (ou mesmo processadores) sem ao menos centenas de transistores**. Um tipo especial de transistor é o Transistor de Efeito de Campo, que permite o controle da condutividade de um de seus canais a partir de um campo elétrico externo.

Em 1990 surgiu a primeira proposta de um Transistor de Efeito de Campo de Spin (ou do inglês, Spin Field Effect Transistor-Spin-FET) ${ }^{42}$. A possibilidade de se controlar a constante de acoplamento de Rashba $(\alpha)$ por campos externos permite o controle do spin de um elétron injetado no 2DEG do poço. Uma vantagem sobre o transistor de campo usual é a possibilidade de detecção e/ou alteração dos estados de spin sem a necessidade de correntes elétricas, o que significa vantagem (de escala, velocidade e tempo) na leitura de dados.

Esquematizamos a idéia original na figura 1.4. A idealização do Spin-FET consistia de dois contatos ferromagnéticos (denotados na figura por FM), um para injetar elétrons no 2DEG e outro para receber estes elétrons de volta. Como $\alpha$ (ver equação 1.2.3) depende do potencial elétrico externo aplicado (gate), podemos controlar o estado final destes elétrons injetados. É fácil ver que ${ }^{1}$, para um poço de comprimento $L$ (distância entre os contatos ferromagnéticos) e para inteiros $n$, então $\alpha$ deve valer $\pi \hbar / 2 n m L$ para que o estado final seja $\uparrow$; para que o estado seja $\downarrow, \alpha=\pi \hbar /(2 n+1) m L$.

O estudo do acoplamento de Rashba, bem como efeitos que possam ocorrer nesse cenário, tornou-se um grande desafio para uma implementação confiável deste dispositivo. O mais interessante é que este dispostivo que apresentei foi recentemente montado com sucesso ${ }^{43} \mathrm{e}$ muitos outros grupos vêm desenvolvendo técnicas e aplicações para este dispositivo ${ }^{44-48}$. A

\footnotetext{
${ }^{* *}$ O Pentium 4, da Intel, trabalha com 55 milhões de transistores CMOS $130 \mathrm{~nm}$.
} 
discrepância de tempo entre a proposta deste dispositivo e sua realização experimental devese a diversos fatores: tempos de coerência de spin, qualidade das estruturas crescidas e das interfaces entre estas estruturas, mecanismos de spin-flip, detecção de portadores polarizados, etc. Por fim, há propostas modificadas e outros dispositivos de spintrônica que motivam outras pesquisas igualmente interessantes, tais como interferômetro de $\operatorname{spin}^{49-51}$, beam-splitters ${ }^{51}$, filtros de spin $^{52}$, memórias baseadas em domínios magnéticos móveis ${ }^{53}$, etc.

\subsection{Dispositivos optoeletrônicos com poços quânticos}

A forma mais utilizada para extrair, de sistemas bem diversificados, informação quantitativamente relevante é interação luz-matéria, e.g., experimentos com fluorescência (determinação de materiais e compostos, química forense, estudo de cadeias de DNA, ... ). Além disso, como comentei há pouco, há muito interesse na implementação de dispositivos baseados em heteroestruturas. Logo de início notaram propriedades interessantes em poços quânticos gerais para construção de detectores ópticos ou para controlar suas propriedades de transporte.

A observação de transições intersub-bandas, dentro da banda de condução, em poços quânticos GaAs/AlGaAs criou a possibilidade de montar novos detectores no infravermeIho ${ }^{54-56}$. Muitos apostam em dispositivos de optoeletrônica usando transições intersub-bandas em poços quânticos devido ao veloz tempo de resposta e à grande força de oscilador ${ }^{56}$. Técnicas de crescimento epitaxial possibilitaram a criação de detectores no infravermelho ${ }^{57} \mathrm{e}$ moduladores ${ }^{58}$ baseados em mecanismos de transições intersub-bandas.

Todos estes estudos, datados da década de 90, são primordialmente baseados em modelos de potenciais infinitos. Dentro do poço, o elétron movimenta-se livremente (como em um 2DEG). Nas paredes do poço, no entanto, a função de onda deve anular-se (quantização fixa nas bordas) e, portanto, fora do poço, a função de onda deve ser nula. Há estruturas que sabemos que isso não ocorre ${ }^{\dagger \dagger}$. Além disso, tratavam-se de modelos de uma única banda. Veremos que implicações têm modelos de uma banda já no capítulo 2. No capítulo 5, veremos quais contribuições outras bandas fornecem à banda de condução.

Atualmente, mecanismos mais sofisticados são criados e apresenta-se muito interesse nesses dispositivos ${ }^{59-61}$. No entanto, muitos destes estudos são para poços quânticos sem dopagem ou para famílias do tipo $I I I$. Há ainda uma grande quantidade de estudos que podemos realizar se modelarmos corretamente a interação elétron-fóton em poços quânticos Zincblende.

$\dagger_{\dagger} \operatorname{lnSb} /$ AllnSb é uma das estruturas em que a função de onda mais "foge" do poço. 


\subsection{Objetivo e divisão dos tópicos}

Primordialmente, o objeivo deste trabalho é modelar a interação entre elétrons de condução e fótons em poços quânticos $I I I-V$ e verificar possíveis efeitos de spintrônica. Me baseio no método $\mathbf{k} \cdot \mathbf{p}$ de oito bandas, a partir do qual é possível deduzir uma equação efetiva para os elétrons de condução. Com este modelo, derivo os acoplamentos elétron-fóton efetivos para a banda de condução levando em conta efeitos das bandas de valência. Todos os cálculos decorrem de expansões em potências do gap do material central, e por esse motivo desenvolvo identidades entre certos operadores que aparecem neste modelo. Ao deduzir a hamiltoniana efetiva estou transformando o modelo de oito bandas (junção do modelo de Kane $8 \times 8$ com as funções envelope) em um modelo efetivo de duas bandas, em que os parâmetros (coeficientes dos acoplamentos, massa efetiva, etc) são descritos em termos das seis bandas que removemos. Obter as interações elétron-fóton efetivas significa não precisar voltar ao modelo $8 \times 8$ para observar transições ópticas nos elétrons de condução.

Mostro algumas soluções da equação efetiva para o caso em que o poço admite duas subbandas (dois estados ligados) e investigo brevemente os elementos de matriz dos acoplamentos elétron-fóton dentro da aproximação de dipolo elétrico. Estudo um destes acoplamentos, que é análogo ao acoplamento elétron-fóton assistido por spin, em mais detalhes por induzir mudança na orientação do spin eletrônico.

O trabalho está dividido como segue:

a) no capítulo 2 deduzimos a equação efetiva para a banda de condução usando uma série de potências do gap do material central;

b) Resolvemos a equação efetiva no capítulo 3 e abordamos alguns dos termos numericamente para verificar brevemente suas contribuições;

c) no capítulo 4, modelamos a interação elétron-fóton para a banda de condução, derivando os acoplamentos elétron-fóton em mesma ordem de aproximação que a equação efetiva do capítulo 2;

d) calculamos no capítulo 5 os elementos de matriz em aproximação de dipolo elétrico das interações elétron-fóton, nos focando em um dos acoplamentos: o assistido por spin;

No capítulo 6 encerramos nossas discussões retomando nossos resultados.

Como muitos dos capítulos apresentam diversos resultados, ao final de cada capítulo incluí uma pequena seção que retoma as principais expressões e raciocínios. Nestes resumos, deixo 
explícito (mesmo que redundante) todos os parâmetros para facilitar futuras aplicações deste texto. Na última página deste capítulo, disponibilizo o "mapa da apresentação" de minha defesa, que pode ajudar um pouco a entender (por uma visão periférica) o caminho que tomaremos ao longo deste trabalho.

\subsection{Comentários sobre notação}

A menos de casos óbvios, denotaremos operadores por letras maiúsculas. Por exemplo, o operador posição $\boldsymbol{R}$ tem como autovalores $\boldsymbol{r}$ - esta notação é a utilizada em boa parte das referências de Mecânica Quântica, em especial na referência adotada na ementa do curso de Mecânica Quântica no IFSC ${ }^{7}$. Manteremos em minúsculo os operadores $k$ nos capítulos 2 e 4 por serem amplamente utilizados desta forma. Manteremos em maiúsculo grandezas que não oferencem nenhuma possibilidade de confusão (parâmetro de kane $\mathcal{P}$ e o potencial $V$ são exemplos de grandezas que não são operadores). 


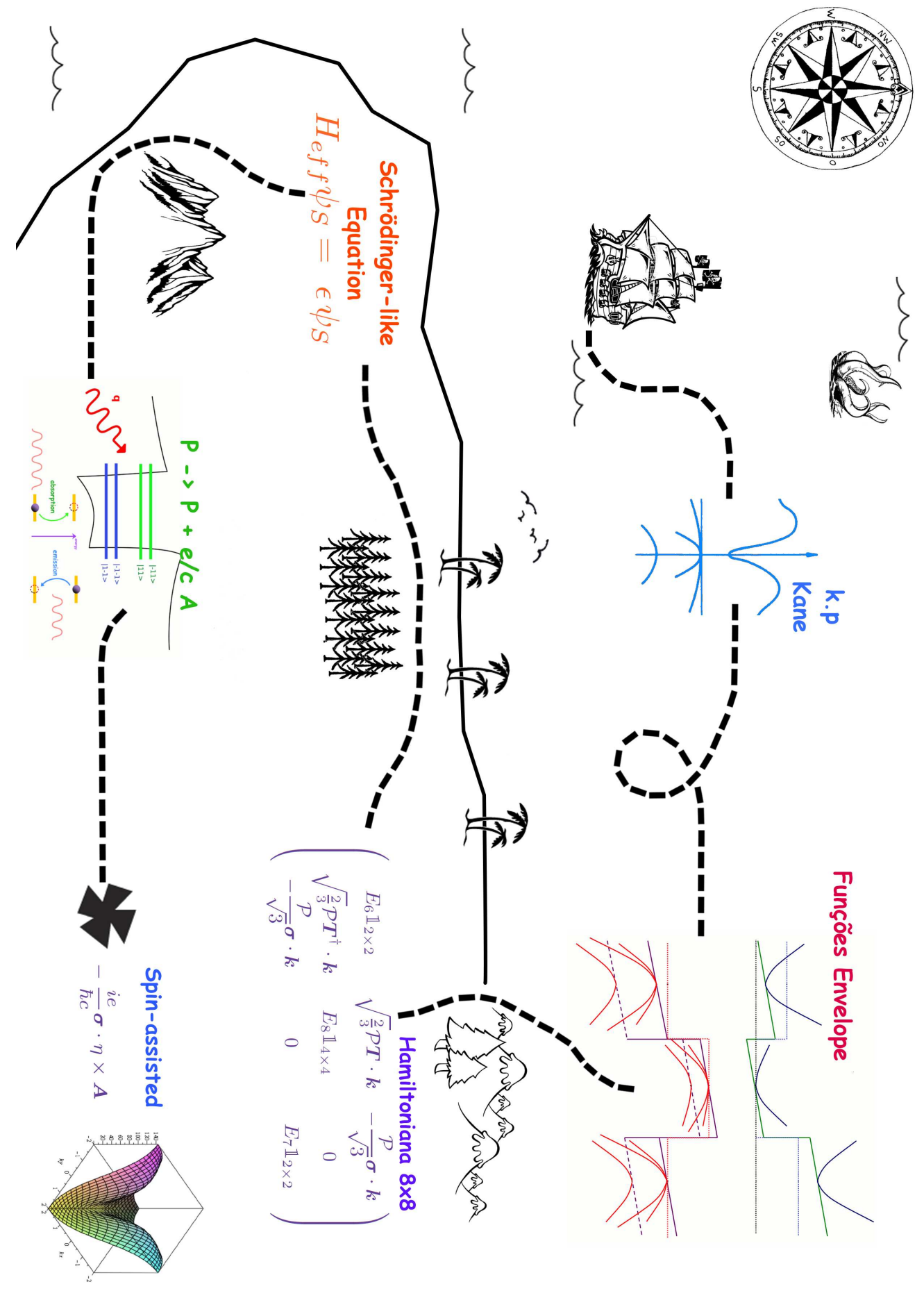




\section{CAPÍTULO 2}

\section{Hamiltoniana Efetiva para Banda de Condução}

I need a tight compadre, who will teach me how to rock My father thinks you are evil, but, man, he can. Jables, em Tenacious D - Kickapoo, 2007.

Vou apresentar um modelo para tratar heteroestruturas com rede Zincblende conhecido como Modelo de Kane de oito bandas (ou simplesmente $8 \times 8$ ). Neste modelo, incluo duas bandas de condução e seis de valência. Mostrarei como é possível desacoplar a banda de condução das bandas de valência utilizando uma técnica baseada em uma linearização, a partir da qual deduzirei, de forma puramente algébrica, uma equação efetiva para a banda de condução. Nesta equação efetiva, veremos as correções para a massa efetiva eletrônica induzidas das bandas de valência, o acoplamento spin-órbita intra e intersub-bandas e termos inéditos independentes de spin.

Para realizar este procedimento e derivar todos os resultados comentados, introduzirei identidades algébricas relacionando os operadores que encontraremos no modelo de Kane. Estas identidades são similares à álgebra que as matrizes de Pauli seguem. O domínio destes operadores é o que garante o sucesso do procedimento.

\subsection{Método $\mathrm{k} \cdot \mathrm{p}$}

Um elétron, de massa de repouso $m$, sob um potencial qualquer é descrito pela equação de $\operatorname{Dirac}^{22}$, que pode ser compreendida como duas equações de autovetores e autovalores matriciais $(2 \times 2)$ acopladas. Desacoplando as equações e as expandindo em ordens de $1 / c$, pode ser mostrado que a solução de uma delas deve se anular ${ }^{22}$. A outra equação (cuja solução não se anula) apresenta diversas correções conforme expandimos nas diferentes ordens de $1 / c$. 
A primeira correção* (de primeira ordem) fornece a famosa interação Zeeman, e é conhecida como equação de Pauli. A segunda correção, da ordem de $1 / c^{2}$, fornece três novos termos: a correção da massa relativística, acoplamento spin-órbita e o termo de Darwin ${ }^{22}$. Esta última hamiltoniana, com apenas o acoplamento spin-órbita, pode ser escrita como

$$
H=\frac{P^{2}}{2 m}+V(\boldsymbol{R})+\frac{\hbar}{4 m^{2} c^{2}} \boldsymbol{\sigma} \times \nabla V(\boldsymbol{R}) \cdot \boldsymbol{P}
$$

em que $\langle\boldsymbol{r}|V(\boldsymbol{R})| \boldsymbol{r}\rangle=V(\boldsymbol{r})$ é o potencial experimentado pelo elétron na posição $\boldsymbol{r}$ e $\boldsymbol{\sigma} \hbar / 2=\left(\sigma_{x}, \sigma_{y}, \sigma_{z}\right) \hbar / 2$ é o operador de spin, com suas componentes sendo as matrizes de Pauli.

Mais especificamente, estamos interessados em estudar um elétron em um sólido cristalino, i.e., sob ação um potencial periódico $V(\boldsymbol{r})$. A periodicidade deste potencial está intimamente ligada com a rede de Bravais ${ }^{62,63}$ deste sólido. A menor região que define a periodicidade do potencial é conhecida como célula unitária. Quando estudamos um sólido cristalino, que tem simetria translacional perfeita ${ }^{62}$, o Teorema de Bloch ${ }^{62,64}$ garante que a função de onda da hamiltoniana 2.1.1, com $V$ periódico na cela unitária, pode ser escrita na forma

$$
\psi_{\boldsymbol{k}}(\boldsymbol{r})=e^{i \boldsymbol{k} \cdot \boldsymbol{r}} u_{\boldsymbol{k}}(\boldsymbol{r})
$$

em que $\boldsymbol{k}$ são vetores na base da rede recíproca ${ }^{62,63}$. As funções $u_{\boldsymbol{k}}(\boldsymbol{r})$, chamadas de funções de célula - conforme a nomenclatura da célula unitária -, herdam a mesma periodicidade de $V(\boldsymbol{r})$. Isto torna $\psi_{\boldsymbol{k}}$ semi-periódica. O conjunto de possíveis vetores $\boldsymbol{k}$ define o que chamamos de espaço recíproco ${ }^{62,64}$.

Lançando mão do teorema 2.1.2, podemos nos perguntar sobre uma hamiltoniana efetiva $H^{\prime}$ tal que

$$
H^{\prime} u_{\boldsymbol{k}}(\boldsymbol{r})=\epsilon(\boldsymbol{k}) u_{\boldsymbol{k}}(\boldsymbol{r})
$$

Para tal, aplico $H$ sobre $\psi_{\boldsymbol{k}}$,

$$
H \psi_{\boldsymbol{k}}(\boldsymbol{r})=\frac{P^{2}}{2 m} \psi_{\boldsymbol{k}}(\boldsymbol{r})+V(\boldsymbol{R}) \psi_{\boldsymbol{k}}(\boldsymbol{r})+\frac{\hbar}{4 m^{2} c^{2}} \boldsymbol{\sigma} \times \nabla V(\boldsymbol{R}) \cdot \boldsymbol{P} \psi_{\boldsymbol{k}}(\boldsymbol{r}) .
$$

Usando a representação de $\boldsymbol{P}$ na base $|\boldsymbol{r}\rangle$,

$$
\begin{aligned}
\boldsymbol{P} \psi_{\boldsymbol{k}}(\boldsymbol{r}) & =-i \hbar \nabla \psi_{\boldsymbol{k}}(\boldsymbol{r})=e^{i \boldsymbol{k} \cdot \boldsymbol{r}}(\hbar \boldsymbol{k}+\boldsymbol{P}) u_{\boldsymbol{k}}(\boldsymbol{r}) \\
P^{2} \psi_{\boldsymbol{k}}(\boldsymbol{r}) & =-\hbar^{2} \nabla^{2} \psi_{\boldsymbol{k}}(\boldsymbol{r})=e^{i \boldsymbol{k} \cdot \boldsymbol{r}}\left(\hbar^{2} k^{2}+P^{2}+2 \boldsymbol{k} \cdot \boldsymbol{P}\right) u_{\boldsymbol{k}}(\boldsymbol{r}) .
\end{aligned}
$$

Assim, a exponencial $e^{i \boldsymbol{k} \cdot \boldsymbol{r}}$ pode ser fatorada em todos os termos da equação 2.1.4. Fatorando

*A correção de ordem zero é simplesmente a equação de Schrödinger sem qualquer alteração 
a exponencial, obtemos a hamiltoniana efetiva

$$
H^{\prime}(\boldsymbol{k})=H_{k p}+W_{k p}(\boldsymbol{k})
$$

em que

$$
H_{k p}=\frac{P^{2}}{2 m}+V(\boldsymbol{R})+\alpha \sigma \times \nabla V(R) \cdot \boldsymbol{P}
$$

e

$$
W_{k p}(\boldsymbol{k})=\frac{\hbar}{m} \boldsymbol{k} \cdot\left\{\boldsymbol{P}+\frac{\hbar}{4 m c^{2}} \boldsymbol{\sigma} \times \nabla V(\boldsymbol{R})\right\} .
$$

Fica claro que $W_{k p}(\mathbf{0})=0$. Uma das opções é então encontrarmos a base que diagonaliza $H_{k p}$ e escrever $W_{k p}$ nesta base. O método $\mathbf{k} \cdot \mathbf{p}$ baseia-se neste princípio: o problema passa a ser visto de forma perturbativa, expandindo em ordens de potência de $k$. O termo $W_{k p}$ dá o nome ao método $\mathbf{k} \cdot \mathbf{p}$, que é frequentemente utilizado para resolver estruturas de bandas tanto de semicondutores ${ }^{64-66}$, como de heteroestruturas semicondutoras ${ }^{41,67}$.

O método $\boldsymbol{k} \cdot \boldsymbol{p}$ descreve as funções de onda num ponto $\boldsymbol{k}$ da zona de Brillouin numa base gerada a partir das funções de onda no ponto $\boldsymbol{k}_{0}$, um ponto qualquer de referência. Como $W_{k p}(\mathbf{0})=0$, escolhi $\boldsymbol{k}_{0}=\mathbf{0}$ como nossa referência, o que me obriga obter de alguma forma as soluções de

$$
H^{\prime}(\mathbf{0})|n \mathbf{0}\rangle=H_{k p}|n \mathbf{0}\rangle=E_{n}(\mathbf{0})|n \mathbf{0}\rangle \text {. }
$$

O que utilizarei, no entanto, são apenas algumas propriedades de simetria destas funções. Supondo que sejam conhecidas as operações de simetria do sólido em questão, podemos é fácil descobrir bases que naturalmente expressam essas simetrias ${ }^{26}$ (isso porque qualquer operação de simetria comuta com a hamiltoniana). Podemos usar a base de $H_{k p}$ para escrever a base de $H^{\prime}$,

$$
|n \boldsymbol{k}\rangle=\sum_{j} C_{j}(\boldsymbol{k}, \mathbf{0})|j, \mathbf{0}\rangle,
$$

em que os coeficientes $C_{j}(\boldsymbol{k}, \mathbf{0})$ são desconhecidos. Aplicando a Hamiltoniana 2.1.6, temos

$$
\begin{gathered}
{\left[H_{k p}+W_{k p}(\boldsymbol{k})\right]|n \boldsymbol{k}\rangle=E_{n}(\boldsymbol{k})|n \boldsymbol{k}\rangle} \\
\sum_{j}\left[H_{k p}+W_{k p}(\boldsymbol{k})\right] C_{j}(\boldsymbol{k}, \mathbf{0})|j, \mathbf{0}\rangle=E_{n}(\boldsymbol{k}) \sum_{j} C_{j}(\boldsymbol{k}, \mathbf{0})|j, \mathbf{0}\rangle .
\end{gathered}
$$

Multiplicando pela esquerda a equação 2.1 .11 por $\langle m|$, chegamos finalmente $a^{1}$

$$
\left[E_{m}(\mathbf{0})-E_{m}(\boldsymbol{k})\right] C_{m}(\boldsymbol{k})+\frac{\hbar}{m} \boldsymbol{k} \cdot \sum_{j}\langle m|\boldsymbol{\pi}| j\rangle C_{j}(\boldsymbol{k})=0
$$


em que defini

$$
\boldsymbol{\pi}:=\boldsymbol{P}+\frac{\hbar}{4 m c^{2}} \boldsymbol{\sigma} \times \nabla V(\boldsymbol{R}) .
$$

Temos em mãos um sistema de equações, cada uma dada por 2.1.12 para diferentes índices $m$. Podemos colocar este sistema na forma matricial e resolvê-lo como um problema de autovalores e autovetores. Os elementos de matriz $\langle m|\boldsymbol{\pi}| j\rangle$ e as energias no ponto $\boldsymbol{\Gamma}$ (i.e., $\boldsymbol{k}=\mathbf{0}$ ) são inseridos manualmente a partir de experimentos realizados com os materiais que desejamos estudar. Por este motivo, chamamos o método $\mathbf{k} \cdot \mathbf{p}$ semiempírico.

\subsection{Modelo de Kane - Bulk}

Como o grupo pontual de simetria do cristal $^{\dagger}$ é conhecido, parte das informações sobre a base que diagonaliza $H^{\prime}(\mathbf{0})$ pode ser obtida a partir de argumentos de simetria ${ }^{27,62}$. Estas informações referem-se à maneira com que estas funções devem transformar-se mediante as operações do grupo de simetria. Na tabela 2.1, listo esta base usando uma notação próxima recorrente em física atômica. As soluções da equação 2.1.9 para um semicondutor de geometria zincblende são bem conhecidas e estudadas ${ }^{1,68}$. Vamos utilizar um conjunto de oito destas soluções para formar uma base com respeito à qual escreveremos uma matriz $8 \times 8$ que representará $H^{\prime}(\boldsymbol{k})$. Estes vetores de base estão listados na tabela 2.1. Como vamos precisar apenas das propriedades de simetria destes vetores, denotamos por $S$ as simetrias esféricas na parte orbital - transforma-se como $f(r)$ - e $X, Y$ e $Z$ as simetriais vetoriais - transformam-se como $x f(r)$. Esta notação é comum em física atômica, em que os orbitais atômicos são as conhecidas funções $s, p_{x}, p_{y}$ e $p_{z}$. A figura 2.1 sintetiza todas estas informações. Apesar de as funções não serem exatamente os orbitais atômicos, suas simetrias são idênticas. Por isso, podemos até argumentar sobre a existência de hibridização $s p^{3}$ nestes semicondutores ${ }^{68}$.

Como aproximação para este tratamento, vamos desprezar o acoplamento spin-órbita no cálculo dos elementos de matriz de $\pi^{\ddagger}$, que então passa a ser igual ao momento linear, i.e., $\boldsymbol{\pi}=\boldsymbol{P}$. Desta identidade, decorrem diversas consequências: $\boldsymbol{\pi}$ compartilha base com a representação irredutível vetorial do grupo pontual do sólido. Por argumentos de teoria de grupos, os únicos elementos de matriz não nulos de $\pi$ são entre estados $|S\rangle$ e $|X\rangle,|Y\rangle$ e $|Z\rangle$. Chamamos de elementos de matriz de Kane os elementos

$$
\mathcal{P}:=-\frac{i \hbar}{m}\left\langle S\left|P_{x}\right| X\right\rangle=-\frac{i \hbar}{m}\left\langle S\left|P_{y}\right| Y\right\rangle=-\frac{i \hbar}{m}\left\langle S\left|P_{z}\right| Z\right\rangle .
$$

†O grupo de simetrial pontual é o grupo com as operações de simetria a menos das translações. O grupo de simetria das redes Zincblende é o grupo $\mathcal{T}_{d}$ ou grupo do tetraedro.

$\ddagger O$ próprio Kane ${ }^{69}$ foi um dos primeiros a desprezar este termo. 


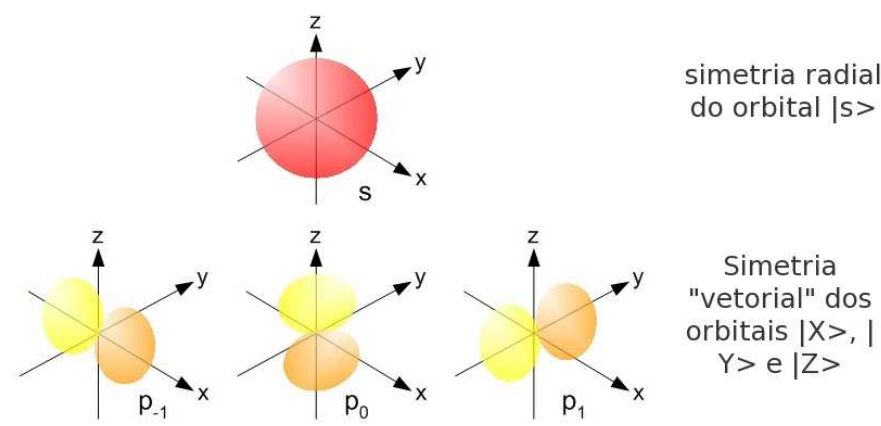

Figura 2.1 - Podemos explorar as simetrias (esférica e vetorial) exibidas pelas funções de onda para anular certos elementos de matriz e diminuir o número de parâmetros do modelo.

Outros elementos de matriz, tais como $\left\langle X\left|P_{z}\right| Z\right\rangle$ ou $\left\langle S\left|P_{y}\right| Z\right\rangle$, são todos nulos (sem qualquer aproximação). Este fato decorre de um teorema de Representação de Grupos bem descrito nas referências de Fazzio e Watari ${ }^{26}$ e Enderlein e Horing ${ }^{68}$. Em geral, $\mathcal{P}$ é um parâmetro extraído de experimentos ${ }^{70}$.

A utilização desta base de oito vetores no método $\mathbf{k} \cdot \mathbf{p}$ e a utilização deste parâmetro empírico dá origem ao que chamamos de modelo padrão de Kane ou modelo de Kane $8 \times 8$. Vamos desprezar alguns acoplamentos dentro das bandas de valência por gerarem efeitos pequenos na banda de condução ${ }^{1,68}$. Colocando a equação 2.1.12 na forma matricial, obtemos de forma explícita

$$
\begin{aligned}
& H=\left[\begin{array}{cccccccc}
E_{6} & 0 & -\frac{1}{\sqrt{2}} \mathcal{P} k_{+} & \sqrt{\frac{2}{3}} \mathcal{P} k_{z} & \frac{1}{\sqrt{6}} \mathcal{P} k_{-} & 0 & -\frac{1}{\sqrt{3}} \mathcal{P} k_{z} & -\frac{1}{\sqrt{3}} \mathcal{P} k_{-} \\
0 & E_{6} & 0 & -\frac{1}{\sqrt{6}} \mathcal{P} k_{+} & \sqrt{\frac{2}{3}} \mathcal{P} k_{z} & \frac{1}{\sqrt{2}} \mathcal{P} k_{-} & -\frac{1}{\sqrt{3}} \mathcal{P} k_{+} & \frac{1}{\sqrt{3}} \mathcal{P} k_{z} \\
-\frac{1}{\sqrt{2}} \mathcal{P} k_{-} & 0 & E_{8} & 0 & 0 & 0 & 0 & 0 \\
\sqrt{\frac{2}{3}} \mathcal{P} k_{z} & -\frac{1}{\sqrt{6}} \mathcal{P} k_{-} & 0 & E_{8} & 0 & 0 & 0 & 0 \\
\frac{1}{\sqrt{6}} \mathcal{P} k_{+} & \sqrt{\frac{2}{3}} \mathcal{P} k_{z} & 0 & 0 & E_{8} & 0 & 0 & 0 \\
0 & \frac{1}{\sqrt{2}} \mathcal{P} k_{+} & 0 & 0 & 0 & E_{8} & 0 & 0 \\
-\frac{1}{\sqrt{3}} \mathcal{P} k_{z} & -\frac{1}{\sqrt{3}} \mathcal{P} k_{-} & 0 & 0 & 0 & 0 & E_{7} & 0 \\
-\frac{1}{\sqrt{3}} \mathcal{P} k_{+} & \frac{1}{\sqrt{3}} \mathcal{P} k_{z} & 0 & 0 & 0 & 0 & 0 & E_{7}
\end{array}\right],
\end{aligned}
$$

em que a diagonal é descrita por

$$
\begin{gathered}
E_{6}=V+\frac{\hbar^{2} k^{2}}{2 m}, \\
E_{8}=V-E_{g}+\frac{\hbar^{2} k^{2}}{2 m}, \\
E_{7}=V-E_{g}-\Delta_{g}+\frac{\hbar^{2} k^{2}}{2 m},
\end{gathered}
$$

e utilizei implicitamente a definição $k_{ \pm}:=k_{x}+i k_{y}$. Como soluções para este modelo, é 
Tabela 2.1 - Tabela com os autovetores no ponto $\boldsymbol{\Gamma}: \boldsymbol{k}=\mathbf{0}$ da zona de Brillouin, soluções da equação 2.1.9. Utilizei esta base para escrever a hamiltoniana de Kane (equação 2.2.2). Escolhi uma base que já diagonaliza automaticamente o acoplamnento spin-órbita: a base do momento angular. A coluna "pontos" evidencia a simetria das funções de Bloch ${ }^{68}$.

\begin{tabular}{|c|c|c|c|}
\hline$j$ & Ponto & $|J, M\rangle$ & $\left|u_{j}\right\rangle$ \\
\hline 1 & $\overline{\Gamma_{6}}$ & $|1 / 2,1 / 2\rangle$ & $i|S \uparrow\rangle$ \\
\hline 2 & $\Gamma_{6}$ & $|1 / 2,-1 / 2\rangle$ & $i|S \downarrow\rangle$ \\
\hline 3 & $\Gamma_{8}$ & $|3 / 2,3 / 2\rangle$ & $\frac{|X \uparrow\rangle+i|Y \uparrow\rangle}{\sqrt{2}}$ \\
\hline 4 & $\Gamma_{8}$ & $|3 / 2,1 / 2\rangle$ & $\sqrt{\frac{2}{3}}|Z \uparrow\rangle-\frac{|X \downarrow\rangle+i|Y \downarrow\rangle}{\sqrt{6}}$ \\
\hline 5 & $\Gamma_{8}$ & $|3 / 2,-1 / 2\rangle$ & $\sqrt{\frac{2}{3}}|Z \downarrow\rangle+\frac{|X \uparrow\rangle-i|Y \uparrow\rangle}{\sqrt{6}}$ \\
\hline 6 & $\Gamma_{8}$ & $|3 / 2,-3 / 2\rangle$ & $\frac{|X \downarrow\rangle-i|Y \downarrow\rangle}{\sqrt{2}}$ \\
\hline 7 & $\Gamma_{7}$ & $|1 / 2,1 / 2\rangle$ & $\frac{-|X \downarrow\rangle-i|Y \downarrow\rangle-|Z \uparrow\rangle}{\sqrt{3}}$ \\
\hline 8 & $\Gamma_{7}$ & $|1 / 2,-1 / 2\rangle$ & $\frac{|Z \downarrow\rangle-|X \uparrow\rangle+i|Y \uparrow\rangle}{\sqrt{3}}$ \\
\hline
\end{tabular}

possível obter ${ }^{68}$ a estrutura de bandas destes materiais. Na figura 2.2 vemos a estrutura de bandas tipicamente observada em semicondutores com estrutura Zincblende. Atualmente, estes parâmetros são fornecidos por meio de fittings experimentais ${ }^{70}$. Destaco principalmente o gap fundamental, $E_{g}$, e o split-off ${ }^{20}, \Delta_{g}$. São exemplos de materiais com tal estrutura de bandas: GaAs (Arseneto de Gálio), InSb (Antimoneto de Índio) e ZnS (Sulfeto de Zinco). Estes exemplos apresentam altos valores para o gap $E_{g}: 1.519 \mathrm{eV}$ para o $\mathrm{GaAs}$ e $0.235 \mathrm{eV}$ para o InSb. Estas energias são centenas de vezes maiores do que as outras escalas de energia envolvidas nestes sistemas.

\subsection{Quebra de simetria e as funções envelope}

A quebra da simetria de translação nos impede, por exemplo, de utilizar o teorema de Bloch. Materiais usualmente apresentam assimetrias naturais, como impurezas. Estes "defeitos", muitos relacionados ao crescimento do material, podem ser tratados de forma especial (e.g., como perturbações). Para um poço quântico (ou qualquer junção semicondutora), no entanto, a quebra de simetria deve-se às diferentes estruturas de banda. A cada material diferente, o elétron experimentará potenciais diferentes (gaps em pontos diferentes, parâmetros de rede, massas efetivas, tudo diferente). Em nosso caso de interesse, todos os materiais têm 


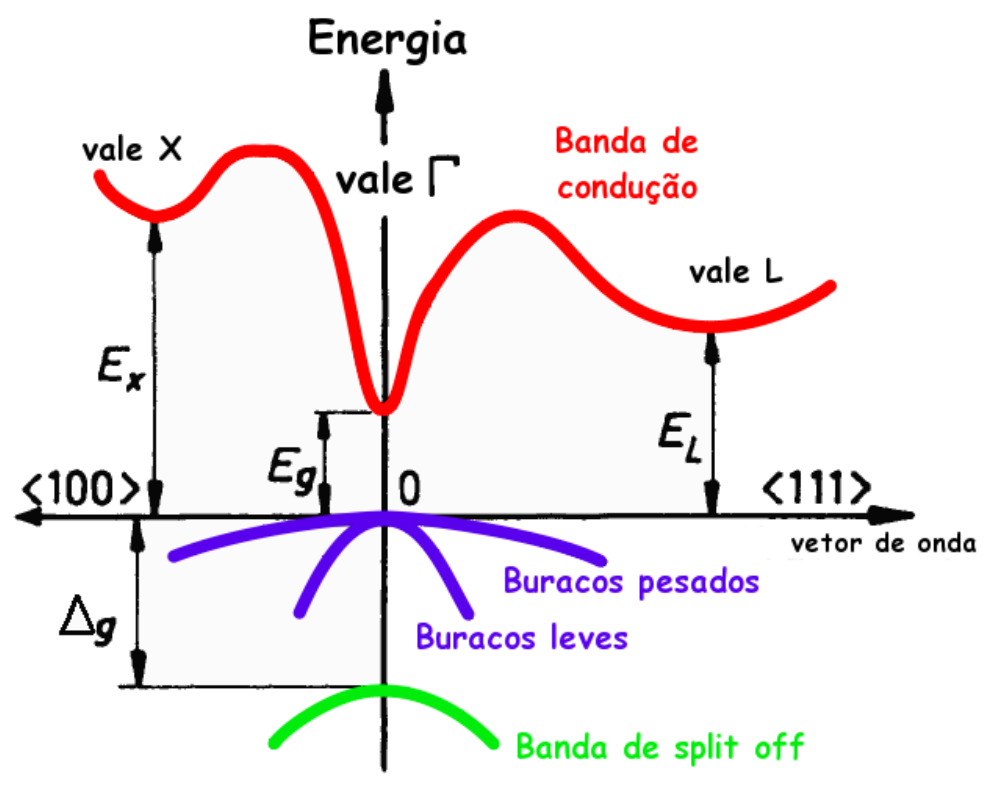

Figura 2.2 - Estrutura de bandas típica para estruturas Zincblende (bulk). O máximo da banda de valência e o mínimo da banda de valência, distantes de $E_{g}$, ocorrem em $\Gamma=\mathbf{0}$ ( $g a p$ direto). Devido à assimetria de inversão da rede, há um split off entre as bandas de valência ${ }^{20}$. Figura traduzida e colorida com base no famoso banco de dados NSM ${ }^{71}$.

gap direto ${ }^{\S}$.

Na interface entre um meio e outro a rede modifica-se - tanto o parâmetro de rede como as espécies químicas envolvidas nos sítios modificam-se. As funções de onda de elétrons no bulk são como gaussianas muito bem localizadas em cada sítio. Dentro de um pequeno volume, existem milhares e milhares de átomos. Podemos visualizar isso na figura 2.3: as pequenas gaussianas que se repetem ao longo de cada estrutura da junção representam as funções de onda de elétrons em bulk. Se cada parte da junção semicondutora estivesse separada, estas seriam as funções de ondas em cada um das estruturas. Como existe uma interface de contato entre elas, impondo limites a cada estrutura, então podemos pensar que as gaussianas ligadas a certa estrutura se anulam fora da sua própria região. Na figura 2.3, associamos cada uma das cores das regiões do poço quântico às pequenas gaussianas: amarelas quando se originam dos sítios do material central (que é o fundo do poço quântico) e vermelhas quando são dos sítios que formam os espaçadores.

Um elétron livre nessa estrutura composta experimenta então o potencial de um poço quântico (como desenhado em preto na figura 2.3). Este perfil de potencial, que chamaremos de potencial estrutural, define uma região que minimiza a energia do elétron, o que gera a possibilidade de confinamento. Um elétron confinado neste poço passa a ocupar apenas os

$\S$ mínimo da banda de condução e o máximo da banda de valência ocorrem em $\boldsymbol{\Gamma}=\mathbf{0}$. 


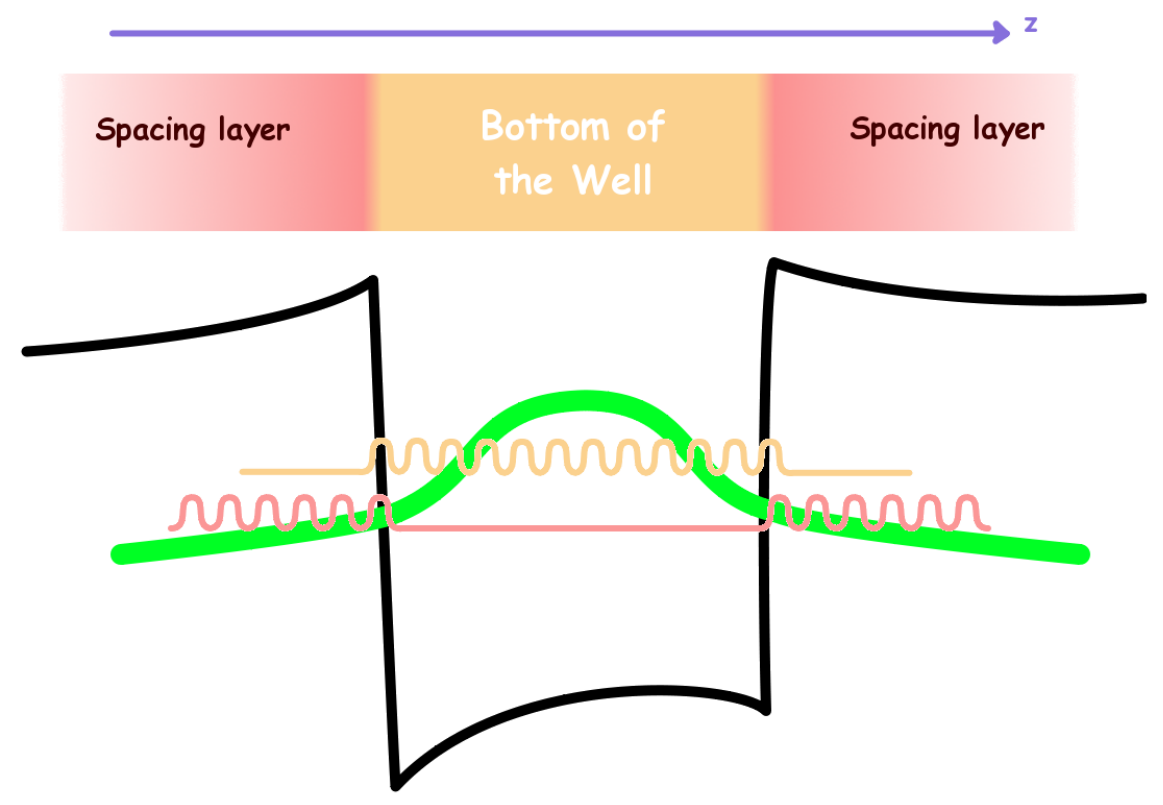

Figura 2.3 - Um poço quântico quebra a simetria de translação na sua direção de crescimento $(z$ na figura). As funções envelope, indicadas em verde, são funções que variam bem mais lentamente que as funções de onda de bulk (representadas pelas pequenas gaussianas vermelhas e amarelas) de qualquer um dos materiais constituintes.

sítios da estrutura central. Ou seja, a função de onda do elétron passa a ser as gaussianas da estrutura central recoberta por um envelope que anula-se fora do poço. Este "envelope" varia muito lentamente se comparado à frequência com que as pequenas gaussianas se repetem. Os estados ligados do poço quântico são bons candidatos a representar estes envelopes.

Toda essa discussão sugere um caminho para nosso estudo: propor uma base formada pelo produto das funções de Bloch do caso bulk e de funções que variam lentamente. Essas funções serão chamadas de funções envelope e mostrarei a qual equação diferencial parcial tais funções devem satisfazer. Veremos a seguir que de fato estas funções envelope são as soluções do poço quântico.

Gostaríamos de resolver

$$
H|\Psi\rangle=\left[H_{0}+\phi(\boldsymbol{R})\right]|\Psi\rangle=E|\Psi\rangle,
$$

em que $\phi(\boldsymbol{R})$ fará o papel do potencial que varia muito lentamente. Veremos logo mais como podemos fazer esta afirmação tornar-se um pouco mais concreta. Conhecemos uma base $\{|j\rangle, j \in \mathbb{N}\}$ tal que

$$
H_{0}|j\rangle=\epsilon_{j}|j\rangle .
$$

Além disso, para simplificar ao máximo, esta base será ortonormal, i.e., $\langle j \mid p\rangle=\delta_{j, p}$. 
Vou usar como função de onda

$$
|\Psi\rangle=\sum_{n} F_{n}(\boldsymbol{R})|n\rangle
$$

em que $F_{n}(\boldsymbol{R})$ a função que gostaríamos de descobrir. Trabalharei apenas com heteroestruturas cujos materiais têm os mesmos grupos de simetria e com funções de onda similares. Como os elétrons devem confinar-se na região central, utilizarei $|j\rangle$ como sendo as funções de onda de bulk do material central. Da equação 2.3.1, temos

$$
H|\Psi\rangle=\sum_{n}\left[H_{0}+\phi(\boldsymbol{R})\right] F_{n}(\boldsymbol{R})|n\rangle
$$

A seguir vou propor uma aproximação que torna o uso desta base uma vantagem significativa.

\subsubsection{Aproximação das funções envelope}

Para construir representações matriciais nesta nova base de funções envelope precisaremos calcular elementos de matriz dos operadores com que trabalharemos. Isso significa calcular integrais com as funções envelope. O domínio destas integrais é todo o espaço, englobando o poço quântico na escala de algumas dezenas de nanometros, ou os sítios atômicos na escala de alguns poucos ångstrons. Neste momento introduzirei uma aproximação que reflete o tamanho do poço quântico e o aprisionamento dos elétrons neste poço.

Primeiramente, vamos verificar se esta nova base é ortogonal, i.e., vejemos os produtos escalares

$$
\left\langle j\left|F_{j}^{\dagger}(\boldsymbol{R}) F_{m}(\boldsymbol{R})\right| m\right\rangle=\int d^{3} r u_{j}^{*}(\boldsymbol{r}) f_{j}^{*}(\boldsymbol{r}) f_{m}(\boldsymbol{r}) u_{m}(\boldsymbol{r}) .
$$

Como $u_{j}$ e $u_{m}$ são funções de cela, têm mesma periodicidade da rede direta. Portanto, podemos escrevê-las como

$$
u_{j}^{*}(\boldsymbol{r}) u_{m}(\boldsymbol{r})=\sum_{\boldsymbol{k} \in \mathcal{R}} g(\boldsymbol{k}) e^{i \boldsymbol{k} \cdot \boldsymbol{r}},
$$

em que $\mathcal{R}$ é o espaço recíproco. Assim, a integral de 2.3.5 torna-se

$$
\left\langle j\left|F_{j}^{\dagger}(\boldsymbol{R}) F_{m}(\boldsymbol{R})\right| m\right\rangle=\sum_{\boldsymbol{k} \in \mathcal{R}} g(\boldsymbol{k}) \int d^{3} r e^{i \boldsymbol{k} \cdot \boldsymbol{r}} f_{j}^{*}(\boldsymbol{r}) f_{m}(\boldsymbol{r}) .
$$

O espaço recíproco sempre está ligado ao espaço direto $\mathcal{D}$ - é o conjunto dos vetores que formam todas as possíveis translações que mantém o sólido invariante. Qualquer vetor $\boldsymbol{r}_{d}$ neste conjunto satisfaz $e^{i \boldsymbol{k} \cdot\left(\boldsymbol{r}_{d}+\boldsymbol{r}\right)}=e^{i \boldsymbol{k} \cdot \boldsymbol{r}}$. Portanto, podemos limitar o domínio de integração 
apenas na célula unitária $\Omega$,

$$
\left\langle j\left|F_{j}^{\dagger}(\boldsymbol{R}) F_{m}(\boldsymbol{R})\right| m\right\rangle=\sum_{\boldsymbol{k} \in \mathcal{R}} g(\boldsymbol{k}) \sum_{\boldsymbol{r}_{d} \in \mathcal{D}} \int_{\Omega} d^{3} r e^{i \boldsymbol{k} \cdot \boldsymbol{r}} f_{j}^{*}\left(\boldsymbol{r}+\boldsymbol{r}_{d}\right) f_{m}\left(\boldsymbol{r}+\boldsymbol{r}_{d}\right) .
$$

Podemos expandir parte do integrando em séries de Taylor para funções de mais de uma variável,

$$
f_{j}^{*}\left(\boldsymbol{r}+\boldsymbol{r}_{d}\right) f_{m}\left(\boldsymbol{r}+\boldsymbol{r}_{d}\right)=f_{j}^{*}\left(\boldsymbol{r}_{d}\right) f_{m}\left(\boldsymbol{r}_{d}\right)+\boldsymbol{r} \cdot \nabla\left(f_{j}^{*}\left[\boldsymbol{r}_{d}\right] f_{m}\left(\boldsymbol{r}_{d}\right)\right)+\mathcal{O}\left(r^{2}\right)
$$

Substituindo esta expansão na equação 2.3.8, obtemos

$$
\begin{array}{r}
\left\langle j\left|F_{j}^{\dagger}(\boldsymbol{R}) F_{m}(\boldsymbol{R})\right| m\right\rangle=\sum_{\boldsymbol{k} \in \mathcal{R}} g(\boldsymbol{k}) \sum_{\boldsymbol{r}_{d} \in \mathcal{D}} \int_{\Omega} d^{3} r e^{i \boldsymbol{k} \cdot \boldsymbol{r}}\left[f_{j}^{*}\left(\boldsymbol{r}_{d}\right) f_{m}\left(\boldsymbol{r}_{d}\right)+\right. \\
\left.\boldsymbol{r} \cdot \nabla\left(f_{j}^{*}\left(\boldsymbol{r}_{d}\right) f_{m}\left(\boldsymbol{r}_{d}\right)\right)+\mathcal{O}\left(r^{2}\right)\right]
\end{array}
$$

Da equação 2.3.6, podemos reconstruir $u_{j}^{*}(\boldsymbol{r}) u_{m}(\boldsymbol{r})$,

$$
\begin{aligned}
\left\langle j\left|F_{j}^{\dagger}(\boldsymbol{R}) F_{m}(\boldsymbol{R})\right| m\right\rangle= & \sum_{\boldsymbol{r}_{d} \in \mathcal{D}} f_{j}^{*}\left(\boldsymbol{r}_{d}\right) f_{m}\left(\boldsymbol{r}_{d}\right) \int_{\Omega} d^{3} r u_{j}^{*}(\boldsymbol{r}) u_{m}(\boldsymbol{r})+ \\
& \sum_{\boldsymbol{r}_{d} \in \mathcal{D}} \nabla\left(f_{j}^{*}\left(\boldsymbol{r}_{d}\right) f_{m}\left(\boldsymbol{r}_{d}\right)\right) \cdot \int_{\Omega} d^{3} r u_{j}^{*}(\boldsymbol{r}) u_{m}(\boldsymbol{r}) \boldsymbol{r}+\mathcal{O}\left(r^{3}\right)
\end{aligned}
$$

Nesta última equação, fica evidente que, se mantermos até primeira ordem em $r$, então a nova base será ortogonal, pois

$$
\int_{\Omega} d^{3} r u_{j}^{*}(\boldsymbol{r}) u_{m}(\boldsymbol{r})=\delta_{j, m}
$$

por suposição. Como a célula unitária tem dimensões tipicamente de alguns nanometros, a soma nos vetores da rede direta pode ser aproximada por uma integral, e dessa forma chegamos finalmente a

$$
\left\langle j\left|F_{j}^{\dagger}(\boldsymbol{R}) F_{m}(\boldsymbol{R})\right| m\right\rangle \approx \delta_{j, m} \int \frac{d^{3} r}{\Omega} f_{j}^{*}(\boldsymbol{r}) f_{m}(\boldsymbol{r}) .
$$

A segunda linha da equação 2.3.11 representa uma estimativa do erro cometido em mais alta ordem,

$$
\Delta:=\int \frac{d^{3} r}{\Omega} \nabla\left[f_{j}^{*}\left(\boldsymbol{r}^{\prime}\right) f_{m}\left(\boldsymbol{r}^{\prime}\right)\right] \cdot \int_{\Omega} d^{3} r u_{j}^{*}(\boldsymbol{r}) u_{m}(\boldsymbol{r}) \boldsymbol{r} .
$$

A quantidade que estamos deixando de lado, $\Delta$, é proporcional às derivadas das funções envelope. Note também que é inversamente proporcional ao volume da célula unitária. Assim, fundamentalmente estamos desprezando variações de primeira ordem nas funções envelope. No capítulo 3.2 estaremos trabalhando com resultados numéricos com os quais é possível 
testar esta hipótese.

E se estivéssemos calculando o elemento de matriz de uma função qualquer, digamos $\Phi(\boldsymbol{r})$, que varie bem lentamente. Neste caso, seguindo os mesmos passos, valerá

$$
\left\langle j\left|F_{j}^{\dagger}(\boldsymbol{R}) \Phi(\boldsymbol{R}) F_{m}(\boldsymbol{R})\right| m\right\rangle \approx \delta_{j, m} \int \frac{d^{3} r}{\Omega} f_{j}^{*}(\boldsymbol{r}) \Phi(\boldsymbol{r}) f_{m}(\boldsymbol{r})+\Delta^{\prime},
$$

em que

$$
\Delta^{\prime}:=\int \frac{d^{3} r}{\Omega} \nabla\left(f_{j}^{*}\left(\boldsymbol{r}^{\prime}\right) \Phi(\boldsymbol{r}) f_{m}\left(\boldsymbol{r}^{\prime}\right)\right) \cdot \int_{\Omega} d^{3} r u_{j}^{*}(\boldsymbol{r}) u_{m}(\boldsymbol{r}) \boldsymbol{r}
$$

é uma estimativa, de mais alta ordem, do erro cometido.

O que chamo de aproximação das funções envelope é a aproximação descrita na equação 2.3.15. Em geral, separarei integrais que envolvem as funções de Bloch e as funções envelope em duas integrais distintas. Isso cria a possibilidade de calcularmos elementos de matriz usando apenas as funções envelopes.

Voltarei a este formalismo na seção 4.4 para estudar o que ocorre na inclusão de campos eletromagnéticos. Por isso, é importante compreendermos este caso, que é bem mais simples e extremamente ilustrativo.

\subsubsection{Equação para as funções envelope}

Com a ajuda da aproximação discutida na seção anterior, podemos calcular elementos de matriz nesta nova base. Com a equação 2.3.4, podemos calcular os elementos de matriz de $H$ e, em seguida, tentar resolver a equação de Schrödinger nesta base nova. Multiplicando 2.3.4 pela esquerda por $\langle j| F_{j}^{\dagger}(\boldsymbol{R})$, obtemos

$$
\begin{aligned}
\left\langle j\left|F_{j}^{\dagger}(\boldsymbol{R}) H\right| \Psi\right\rangle & =\sum_{n}\left\langle j\left|F_{j}^{\dagger}(\boldsymbol{R})\left[H_{0}+\phi(\boldsymbol{R})\right] F_{n}(\boldsymbol{R})\right| n\right\rangle \\
& =\sum_{n}\left[\left\langle j\left|F_{j}^{\dagger}(\boldsymbol{R}) H_{0} F_{n}(\boldsymbol{R})\right| n\right\rangle+\left\langle j\left|F_{j}^{\dagger}(\boldsymbol{r}) \phi(\boldsymbol{R}) F_{n}(\boldsymbol{R})\right| n\right\rangle\right]
\end{aligned}
$$

A parte que envolve o potencial $\phi(r)$ separa-se da forma como já vimos na equação 2.3.15. Como sabemos como $H_{0}$ age sobre os vetores $|j\rangle$, então podemos comutar $H_{0}$ com $F_{n}(R)$ para ganhar alguma vantagem,

$$
\left\langle j\left|F_{j}^{\dagger}(\boldsymbol{R}) H_{0} F_{n}(\boldsymbol{R})\right| n\right\rangle=\left\langle j\left|F_{j}^{\dagger}(\boldsymbol{R}) F_{n}(\boldsymbol{R}) H_{0}\right| n\right\rangle+\left\langle j\left|F_{j}^{\dagger}(\boldsymbol{R})\left[H_{0}, F_{n}(\boldsymbol{R})\right]\right| n\right\rangle .
$$


Como $F_{n}(R)$ é uma função do operador posição $(R)$, então

$$
\left[H_{0}, F_{n}(\boldsymbol{R})\right]=\frac{1}{2 m}\left[P^{2}, F_{n}(\boldsymbol{R})\right]=-\frac{\hbar^{2}}{2 m} \nabla^{2} F_{n}(\boldsymbol{R})-i \frac{\hbar}{m} \nabla F_{n}(\boldsymbol{R}) \cdot \boldsymbol{P} .
$$

Substituindo o comutador que desenvolvemos na equação 2.3.19 na equação 2.3.18,

$$
\begin{aligned}
&\left\langle j\left|F_{j}^{\dagger}(\boldsymbol{R}) H_{0} F_{n}(\boldsymbol{R})\right| n\right\rangle=\epsilon_{n}\left\langle j\left|F_{j}^{\dagger}(\boldsymbol{R}) F_{n}(\boldsymbol{R})\right| n\right\rangle- \\
& \frac{\hbar^{2}}{2 m}\left\langle j\left|F_{j}^{\dagger}(\boldsymbol{R}) \nabla^{2} F_{n}(\boldsymbol{R})\right| n\right\rangle-i \frac{\hbar}{m}\left\langle j\left|F_{j}^{\dagger}(\boldsymbol{R}) \nabla F_{n}(\boldsymbol{R}) \cdot \boldsymbol{P}\right| n\right\rangle .
\end{aligned}
$$

Usando a aproximação 2.3.13 na equação 2.3.20, obtemos finalmente

$$
\begin{aligned}
& \sum_{n}\left\langle j\left|F_{j}^{\dagger}(\boldsymbol{R}) H F_{n}(\boldsymbol{R})\right| n\right\rangle= \\
& \sum_{n}\left[\int \frac{d^{3} r}{\Omega} \delta_{j, n}\left\{\epsilon_{n} f_{j}^{*}(\boldsymbol{r}) f_{n}(\boldsymbol{r})-\frac{\hbar^{2}}{2 m} f_{j}^{*}(\boldsymbol{r}) \nabla^{2} f_{n}(\boldsymbol{r})\right\}-\frac{\hbar}{m} f_{j}^{*}(\boldsymbol{r}) \nabla f_{n}(\boldsymbol{r}) \cdot\langle j|\boldsymbol{P}| n\rangle\right] \\
& =\sum_{n} \delta_{j, n} \int \frac{d^{3} r}{\Omega}(E-\phi(\boldsymbol{r})) f_{j}^{*}(\boldsymbol{r}) f_{n}(\boldsymbol{r}) .
\end{aligned}
$$

Esta última equação já define, por si só, uma equação para determinarmos as funções envelope. Note que trata-se de um problema de autovalores, assim como o problema original, mas com alguns termos diferentes. Para finalizar e explicitar o que quero mostrar, vou reescrever 2.3.21 na forma

$$
\begin{aligned}
\sum_{n}\left[\int \frac { d ^ { 3 } r } { \Omega } \delta _ { j , n } \left\{\left(\epsilon_{n}+\phi(\boldsymbol{r})-E\right) f_{j}^{*}(\boldsymbol{r}) f_{n}(\boldsymbol{r})-\right.\right. \\
\left.\left.\frac{\hbar^{2}}{2 m} f_{j}^{*}(\boldsymbol{r}) \nabla^{2} f_{n}(\boldsymbol{r})\right\}-\frac{\hbar}{m} f_{j}^{*}(\boldsymbol{r}) \nabla f_{n}(\boldsymbol{r}) \cdot\langle j|\boldsymbol{P}| n\rangle\right]=0 .
\end{aligned}
$$

Esta última equação pode ser mapeada na equação 2.1.11 do método $\mathrm{k} \cdot \mathrm{p}$ se identificarmos $k \rightarrow-i \nabla$. Assim, podemos representar a equação 2.3.22 matricialmente em uma base com 8 vetores. Esta representação é idêntica à representação matricial do modelo $\mathbf{k} \cdot \mathbf{p}$ para bulk.

\subsection{Hamiltoniana de Kane para heteroestruturas}

Como vimos nas seções precedentes, é simples aplicar o método das funções envelope para descrever uma heteroestrutura, e poucas modificações serão feitas a partir do modelo de Bulk (de volta, seção 2.2). Por fim, a matriz de Kane, equação 2.2.2, continuará válida se 
redefinirmos as diagonais da matriz por

$$
\begin{gathered}
E_{6}=V+h_{6}+\frac{\hbar^{2} k^{2}}{2 m}, \\
E_{8}=V-h_{8}-E_{g}+\frac{\hbar^{2} k^{2}}{2 m}, \\
E_{7}=V-h_{7}-E_{g}-\Delta_{g}+\frac{\hbar^{2} k^{2}}{2 m} .
\end{gathered}
$$

Além disso, utilizarei implicitamente a definição de $V$, o potential de Hartree. A figura 2.4 evidencia os potenciais $E_{j}$. Para esta construção, usamos $h_{j}=\delta_{j} h_{w}$, em que $h_{w}$ é um perfil de poço quântico com uma unidade de profundidade e $\delta_{j}$ são constantes positivas que dão a profundidade do poço. Ao ajustar o sinal e o valor absoluto destes parâmetros, podemos trabalhar com poços de tipo $I I$ e $I I I$. No entanto, trabalharei apenas com poços do tipo $I$, em que $\delta_{j}>0$.

Vou definir $h_{w}$ usando a função $\mathfrak{H}(z)$ degrau de Heaviside,

$$
h_{w}(z):=1-\mathfrak{H}\left(z+a_{0}\right)+\mathfrak{H}\left(z-a_{0}\right)
$$

Há diversas vantagens (para um tratamento analítico) em usar estas funções. Explorarei algumas de suas propriedades no capítulo 3.

Ainda da figura 2.4 é possível relacionar as grandezas $\delta_{7}$ e $\delta_{8}$ com os gaps dos materiais. Por considerações puramente geométricas,

$$
\begin{gathered}
\delta_{8}=E_{w}-E_{g}-\delta_{6} \\
\delta_{7}=E_{w}+\Delta_{w}-E_{g}-\Delta_{g}-\delta_{6}=E_{w}-E_{g}+\Delta_{w}-\Delta_{g}-\delta_{6} .
\end{gathered}
$$

Como vamos nos focar na banda de condução, é interessante descrever todos os parâmetros em termos de $\delta_{6}$.

A Hamiltoniana de Kane, explicitamente na equação 2.2.2, pode ser reescrita como

$$
H=\left(\begin{array}{ccc}
E_{6} \mathbb{1}_{2 \times 2} & \sqrt{\frac{2}{3}} \mathcal{P} \boldsymbol{T} \cdot \boldsymbol{k} & -\frac{\mathcal{P}}{\sqrt{3}} \boldsymbol{\sigma} \cdot \boldsymbol{k} \\
\sqrt{\frac{2}{3}} \mathcal{P} \boldsymbol{T}^{\dagger} \cdot \boldsymbol{k} & E_{8} \mathbb{1}_{4 \times 4} & 0 \\
-\frac{\mathcal{P}}{\sqrt{3}} \boldsymbol{\sigma} \cdot \boldsymbol{k} & 0 & E_{7} \mathbb{1}_{2 \times 2}
\end{array}\right),
$$




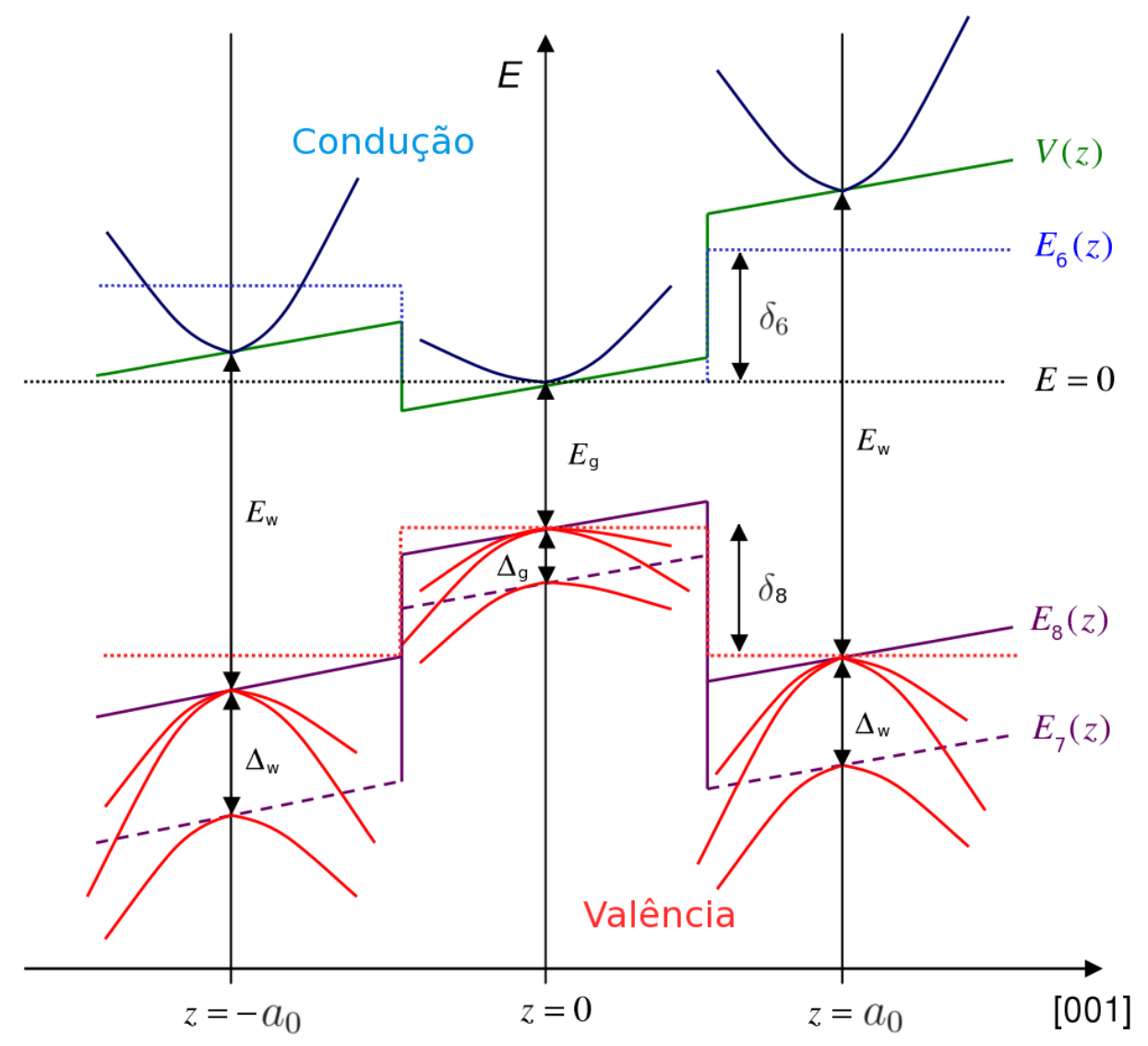

Figura 2.4 - Perfil dos potenciais e seus parâmetros. Trabalharei apenas com poços quânticos do tipo $l$, em que os perfis de potencial não se cruzam e os das bandas de valência formam barreiras. À esquerda, vemos a estrutura de bandas, nas proximidades de $\boldsymbol{\Gamma}$, para o material central. Os perfis $E_{6}, E_{7}$ e $E_{8}$ estão esquematizados de acordo com as distâncias entre os mínimos e máximos das bandas de condução e valência, respectivamente.

em que $\sigma$ é um vetor de matrizes de Pauli e definimos ${ }^{\top}$ as três matrizes

$$
\begin{aligned}
& T_{x}=\frac{1}{2}\left[\begin{array}{cccc}
-\sqrt{3} & 0 & 1 & 0 \\
0 & -1 & 0 & \sqrt{3}
\end{array}\right], \\
& T_{y}=\frac{-i}{2}\left[\begin{array}{cccc}
\sqrt{3} & 0 & -1 & 0 \\
0 & 1 & 0 & \sqrt{3}
\end{array}\right], \\
& T_{z}=\left[\begin{array}{llll}
0 & 1 & 0 & 0 \\
0 & 0 & 1 & 0
\end{array}\right] .
\end{aligned}
$$

A equação de Schrödinger torna-se

$$
H \Psi=\epsilon \Psi
$$

IHá diferenças entre algumas referências. Definimos desta forma para facilitar algumas contas presentes na seção 2.5 . 
em que defini o vetor $\Psi=(\psi, \phi, \chi)^{T}$. Esta separação é claramente a mesma divisão que fizemos no caso Bulk, chamando $\psi$ de banda de condução (duas componentes) e chamando $\phi$ (quatro componentes) e $\chi$ (duas componentes) de banda de valência. Notavelmente é possível escrever, usando as equações 2.4 .5 e 2.4.7, três novas equações diferenciais acopladas,

$$
\begin{gathered}
\sqrt{\frac{2}{3}} \mathcal{P} \boldsymbol{T} \cdot \boldsymbol{k} \phi-\frac{P}{\sqrt{3}} \boldsymbol{\sigma} \cdot \boldsymbol{k} \chi=\left(\epsilon-E_{6}\right) \psi, \\
\sqrt{\frac{2}{3}} \mathcal{P} \boldsymbol{T}^{\dagger} \cdot \boldsymbol{k} \psi=\left(\epsilon-E_{8}\right) \phi \\
-\frac{\mathcal{P}}{\sqrt{3}} \boldsymbol{\sigma} \cdot \boldsymbol{k} \psi=\left(\epsilon-E_{7}\right) \chi
\end{gathered}
$$

Como estamos verdadeiramente interessados em $\psi$, isolei $\phi$ e $\chi$ nas equações $2.4 .8 \mathrm{~b}$ e 2.4.8c,

$$
\begin{gathered}
\phi=\frac{1}{\epsilon-E_{8}} \sqrt{\frac{2}{3}} \mathcal{P} \boldsymbol{T}^{\dagger} \cdot \boldsymbol{k} \psi, \\
\chi=\frac{-1}{\epsilon-E_{7}} \frac{\mathcal{P}}{\sqrt{3}} \boldsymbol{\sigma} \cdot \boldsymbol{k} \psi .
\end{gathered}
$$

Substituindo as equação $2.4 .9 \mathrm{a}$ e $2.4 .9 \mathrm{~b}$ na equação $2.4 .8 \mathrm{a}$, obtemos

$$
\frac{2 \mathcal{P}^{2}}{3} \boldsymbol{T} \cdot \boldsymbol{k} \frac{1}{\epsilon-E_{8}} \boldsymbol{T}^{\dagger} \cdot \boldsymbol{k} \psi+\frac{\mathcal{P}^{2}}{3} \boldsymbol{\sigma} \cdot \boldsymbol{k} \frac{1}{\epsilon-E_{7}} \boldsymbol{\sigma} \cdot \boldsymbol{k} \psi=\left(\epsilon-E_{6}\right) \psi
$$

que é uma equação efetiva e desacoplada para a banda de condução. Como $\epsilon$, o autovalor da equação 2.4.7, está presente em ambos os lados da equação 2.4.10, ou seja, não se trata de uma equação de autovalores e autovetores

Entretanto, podemos obter equações que lembram a equação de Schrödinger se assumirmos que $E_{g}$ é a maior energia envolvida e realizarmos expansões em potências de $1 / E_{g}$. Antes, precisamos conhecer um pouco melhor as matrizes dadas pelas equações 2.4.6. Só então seremos capazes de manipular estas expressões algebricamente e é a esta tarefa que nos dedicaremos até o final deste capítulo. Algo bem similar ocorre com a equação de Dirac: podemos separar as quatro componentes em dois grupos de duas, obtendo no entanto hamiltonianas efetivas que dependem das energias (o apêndice B mostra este procedimento).

\section{5 Álgebra das matrizes $T_{j}$}

Como comentei anteriormente, as três matrizes introduzidas nas equações 2.4.6 apresentam caracterísiticas interessantes, características estas comparáveis às matrizes de Pauli. Vou utilizar estas características para desenvolver um pouco mais a equação efetiva 2.4.10.

Primeiramente, podemos notar que o produto entre estas matrizes obedece as duas se- 
guintes regras:

$$
\begin{gathered}
T_{j} T_{j}^{\dagger}=\mathbb{1}_{2 \times 2} . \\
T_{j} T_{k}^{\dagger}=-\frac{i}{2} \sum_{p=1}^{3} \epsilon_{j k p} \sigma_{p}, \quad j \neq k .
\end{gathered}
$$

Ou seja, é verdade que

$$
T_{j} T_{k}^{\dagger}+T_{k} T_{j}^{\dagger}=0
$$

Podemos definir um tipo de anticomutação entre estas matrizes (acertando, é claro, a transposição e a conjugação).

Suponha dois operadores $\boldsymbol{A}$ e $\boldsymbol{B}$, tal que $\left[A_{j}, B_{k}\right] \neq 0 \forall j, k$, então

$$
\begin{aligned}
& \boldsymbol{T} \cdot \boldsymbol{A} \boldsymbol{T} \cdot \boldsymbol{B}=\sum_{j=1}^{3} A_{j} B_{j} T_{j} T_{j}^{\dagger}+\sum_{j \neq k} A_{j} B_{k} T_{j} T_{k}^{\dagger}= \\
& \mathbb{1}_{2 \times 2} \sum_{j=1}^{3} A_{j} B_{j}-\frac{i}{2} \sum_{j \neq k} A_{j} B_{k} \sum_{p=1}^{3} \epsilon_{j k p} \sigma_{p}=\mathbb{1}_{2 \times 2} \boldsymbol{A} \cdot \boldsymbol{B}-\frac{i}{2} \sum_{j k p} \epsilon_{j k p} A_{j} B_{k} \sigma_{p} .
\end{aligned}
$$

Identificando na equação 2.5.4 um produto vetorial, vou reescrevê-la de forma mais compacta:

$$
\boldsymbol{T} \cdot \boldsymbol{A T} \cdot \boldsymbol{B}=\mathbb{1}_{2 \times 2} \boldsymbol{A} \cdot \boldsymbol{B}-\frac{i}{2} \boldsymbol{\sigma} \cdot \boldsymbol{A} \times \boldsymbol{B} .
$$

No caso em que $\left[A_{j}, B_{k}\right]=0$, então

$$
\begin{aligned}
\sum_{j \neq k} A_{j} B_{k} T_{j} T_{k}^{\dagger} & =\frac{1}{2}\left[\sum_{j \neq k} A_{j} B_{k} T_{j} T_{k}^{\dagger}+\sum_{j \neq k} A_{k} B_{j} T_{k} T_{j}^{\dagger}\right] \\
= & \frac{1}{2}\left[\sum_{j \neq k} A_{j} B_{k} T_{j} T_{k}^{\dagger}+\sum_{j \neq k} A_{j} B_{k} T_{k} T_{j}^{\dagger}\right]=\sum_{j \neq k} A_{j} B_{k} \frac{\left[T_{j} T_{k}^{\dagger}+T_{k} T_{j}^{\dagger}\right]}{2}=0 .
\end{aligned}
$$

Portanto, das equações 2.5 .4 e 2.5 .6 obtemos

$$
\boldsymbol{T} \cdot \boldsymbol{A T} \cdot \boldsymbol{B}=\mathbb{1}_{2 \times 2} \boldsymbol{A} \cdot \boldsymbol{B}
$$

Note que a equação 2.5.7 vale sempre que $\left[A_{j}, B_{k}\right]=0 \forall j, k$. Por exemplo, se $\boldsymbol{A}=\boldsymbol{B}$, então esta condição vale e obtemos o resultado

$$
\boldsymbol{T} \cdot \boldsymbol{A T} \cdot \boldsymbol{A}=A^{2}
$$

A equação 2.5 .8 será muito importante para os desenvolvimentos seguintes, tal como a equação 2.5.5. Note que o resultado 2.5 .5 é o caso mais geral, e as equações 2.5 .8 e 2.5 .7 podem ser deduzidas a partir do cálculo explícito de $\boldsymbol{A} \times \boldsymbol{B}$, que, no caso em que $\left[A_{j}, B_{k}\right]=0$, anula-se. 
O resultado 2.5 .5 é muito similar ao conhecidíssimo resultado das matrizes de Pauli,

$$
\boldsymbol{\sigma} \cdot \boldsymbol{A} \boldsymbol{\sigma} \cdot \boldsymbol{B}=\boldsymbol{A} \cdot \boldsymbol{B}+i \boldsymbol{\sigma} \cdot \boldsymbol{A} \times \boldsymbol{B}
$$

Todas estas propriedades serão altamente aproveitadas.

\subsubsection{Desenvolvimento dos operadores escalares}

O grande interesse é trabalhar com operadores do tipo $\boldsymbol{T} \cdot \boldsymbol{k}$, em que $k_{j}$ é representado por uma derivada da $j$-ésima coordenada. Gostaríamos de saber então como age o operador $\boldsymbol{T} \cdot \boldsymbol{k} \boldsymbol{T}^{\dagger} \cdot \boldsymbol{k}$. Para isso, tome $f$ como uma função teste, então, usando a equação 2.5.8,

$$
\boldsymbol{T} \cdot \boldsymbol{k} \boldsymbol{T}^{\dagger} \cdot \boldsymbol{k} f=k^{2} f
$$

Se, no entanto, temos $\boldsymbol{T} \cdot \boldsymbol{k} g \boldsymbol{T}^{\dagger} \cdot \boldsymbol{k}$ com $g$ sendo um operador escalar tal que $\left[k_{j}, g\right] \neq 0$, então, pela regra do produto (de derivadas),

$$
\boldsymbol{T} \cdot \boldsymbol{k} g \boldsymbol{T}^{\dagger} \cdot \boldsymbol{k} f=g \boldsymbol{T} \cdot \boldsymbol{k} \boldsymbol{T}^{\dagger} \cdot \boldsymbol{k} f+\boldsymbol{T} \cdot(\boldsymbol{k} g) \boldsymbol{T}^{\dagger} \cdot \boldsymbol{k} f
$$

em que $(\boldsymbol{k} g)$ significa que $\boldsymbol{k}$ atua em $g$ e não no que procede, ou seja, $(\boldsymbol{k} g) \rightarrow-i \nabla g$. Sendo assim,

$$
g \boldsymbol{T} \cdot \boldsymbol{k} \boldsymbol{T}^{\dagger} \cdot \boldsymbol{k} f+\boldsymbol{T} \cdot(\boldsymbol{k} g) \boldsymbol{T}^{\dagger} \cdot \boldsymbol{k} f=g k^{2} f+(\boldsymbol{k} g) \cdot \boldsymbol{k} f-\frac{i}{2} \boldsymbol{\sigma} \cdot(\boldsymbol{k} g) \times \boldsymbol{k} f .
$$

Assim, obtemos

$$
\boldsymbol{T} \cdot \boldsymbol{k} g \boldsymbol{T}^{\dagger} \cdot \boldsymbol{k} f=\left[g k^{2}+(\boldsymbol{k} g) \cdot \boldsymbol{k}-\frac{i}{2} \boldsymbol{\sigma} \cdot(\boldsymbol{k} g) \times \boldsymbol{k}\right] f .
$$

A equação 2.5 .13 fornece a ação de $\boldsymbol{T} \cdot \boldsymbol{k} g \boldsymbol{T}^{\dagger} \cdot \boldsymbol{k}$ sobre qualquer função.

\subsubsection{Aplicação com ponto quântico}

Além do poço quântico, comentamos no capítulo 1 a existência de outras formas de confinamentos, como o fio quântico ou o ponto quântico. Neste último caso, podemos usar $E_{6}=E_{8}=E_{7}=V$, em que o potencial $V$ depende de $\boldsymbol{r}$, i.e., das três dimensões ${ }^{72}$. Vou deduzir então uma equação efetiva para a banda de condução sem aproximações, apenas usando a álgebra apresentada nas seções precedentes. Servirá muito bem para compreendermos, na prática, como usar o resultado 2.5.13.

Da equação 2.4.10, usaremos, no que segue, 2.5.13 para manipular as energias nos deno- 
minadores usando a relação 2.5.5. Primeiramente,

$$
\begin{gathered}
\boldsymbol{\sigma} \cdot \boldsymbol{k} \frac{1}{\epsilon-V-E_{g}-\Delta_{g}} \boldsymbol{\sigma} \cdot \boldsymbol{k}=\boldsymbol{\sigma} \cdot\left(\boldsymbol{k} \frac{1}{\epsilon-V-E_{g}-\Delta_{g}}\right) \boldsymbol{\sigma} \cdot \boldsymbol{k}+\frac{1}{\epsilon-V-E_{g}-\Delta_{g}} \boldsymbol{\sigma} \cdot \boldsymbol{k} \boldsymbol{\sigma} \cdot \boldsymbol{k} \\
=\boldsymbol{\sigma} \cdot\left(\boldsymbol{k} \frac{1}{\epsilon-V-E_{g}-\Delta_{g}}\right) \boldsymbol{\sigma} \cdot \boldsymbol{k}+\frac{1}{\epsilon-V-E_{g}-\Delta_{g}} k^{2} \\
=\left(\boldsymbol{k} \frac{1}{\epsilon-V-E_{g}-\Delta_{g}}\right) \cdot \boldsymbol{k}+i \boldsymbol{\sigma} \cdot\left(\boldsymbol{k} \frac{1}{\epsilon-V-E_{g}-\Delta_{g}}\right) \times \boldsymbol{k}+\frac{1}{\epsilon-V-E_{g}-\Delta_{g}} k^{2} .
\end{gathered}
$$

Como só $V$ não comuta com $\boldsymbol{k}$, temos

$$
\left(\boldsymbol{k} \frac{1}{\epsilon-V-E_{g}-\Delta_{g}}\right)=\frac{1}{\left(\epsilon-V-E_{g}-\Delta_{g}\right)^{2}}(\boldsymbol{k} V) .
$$

Desta forma, a equação 2.5.14 torna-se

$$
\begin{aligned}
\boldsymbol{\sigma} \cdot \boldsymbol{k} \frac{1}{\epsilon-V-E_{g}-\Delta_{g}} \boldsymbol{\sigma} \cdot \boldsymbol{k}=\frac{1}{\left(\epsilon-V-E_{g}-\Delta_{g}\right)^{2}}(\boldsymbol{k} V) \cdot \boldsymbol{k}+ \\
\frac{i}{\left(\epsilon-V-E_{g}-\Delta_{g}\right)^{2}} \boldsymbol{\sigma} \cdot(\boldsymbol{k} V) \times \boldsymbol{k}+\frac{1}{\epsilon-V-E_{g}-\Delta_{g}} k^{2} .
\end{aligned}
$$

Similarmente, usando a equação 2.5.5,

$$
\begin{aligned}
\boldsymbol{T} \cdot \boldsymbol{k} & \frac{2}{\epsilon-V-E_{g}} \boldsymbol{T}^{\dagger} \cdot \boldsymbol{k}=\boldsymbol{T} \cdot\left(\boldsymbol{k} \frac{2}{\epsilon-V-E_{g}}\right) \boldsymbol{T}^{\dagger} \cdot \boldsymbol{k}+\frac{2}{\epsilon-V-E_{g}} \boldsymbol{T} \cdot \boldsymbol{k} \boldsymbol{T}^{\dagger} \cdot \boldsymbol{k} \\
& =\frac{2}{\left(\epsilon-V-E_{g}\right)^{2}}(\boldsymbol{k} V) \cdot \boldsymbol{k}-\frac{i}{\left(\epsilon-V-E_{g}\right)^{2}} \boldsymbol{\sigma} \cdot(\boldsymbol{k} V) \times \boldsymbol{k}+\frac{2}{\epsilon-V-E_{g}} k^{2} .
\end{aligned}
$$

Reunindo os termos, obtemos por fim a equação

$$
\begin{aligned}
& \frac{\mathcal{P}^{2}}{3}\left[\left\{\frac{2}{\epsilon-V-E_{g}}+\frac{1}{\epsilon-V-E_{g}-\Delta_{g}}\right\} k^{2}+\right. \\
& \qquad\left\{\frac{2}{\left(\epsilon-V-E_{g}\right)^{2}}+\frac{1}{\left(\epsilon-V-E_{g}-\Delta_{g}\right)^{2}}\right\}(\boldsymbol{k} V) \cdot \boldsymbol{k} \\
& \left.\quad-i\left\{\frac{1}{\left(\epsilon-V-E_{g}\right)^{2}}-\frac{1}{\left(\epsilon-V-E_{g}-\Delta_{g}\right)^{2}}\right\} \boldsymbol{\sigma} \cdot(\boldsymbol{k} V) \times \boldsymbol{k}\right] \psi=(\epsilon-V) \psi
\end{aligned}
$$

Esta equação é utilizada para pontos quânticos ${ }^{72}$ e não apresenta aproximações, ao preço da presença de $\epsilon$ na hamiltoniana efetiva, o que dificulta enormemente o problema.

As três diferentes contribuições do lado esquerdo da equação 2.5.18, advindas das bandas de valência, merecem comentários: a primeira é como uma renormalização da massa efetiva; a segunda é um acoplamento momento-campo elétrico, já que envolve o produto de $\boldsymbol{k}$ e da derivada do potencial $\boldsymbol{k} V$, que pode ser compreendido como um campo elétrico gerado por 
$V$; o último é um acoplamento spin-órbita. Note que não há nenhum termo similar ao termo de Darwin (proporcional à segunda derivada do potencial $V$ ) na equação 2.5.18. Isso porque o termo de Darwin é uma contribuição que ocorre da correção da normalização de $\psi$ devido às bandas de valência. Todos estes termos aparecem a partir da seção 2.7.1.

\section{$2.6 E_{g}$ como maior energia envolvida}

Os denominadores de energia que aparecem na equação 2.4 .10 são complicados: o ideal é trabalhar com operadores linearizados. Por isso vou expandi-los em ordens de potências de $1 / E_{g}$. Uma das frações, até segunda ordem em $1 / E_{g}$, pode ser escrita como

$$
\begin{aligned}
\frac{1}{\epsilon-V+h_{8}+E_{g}-\frac{\hbar^{2} k^{2}}{2 m}}=\frac{1}{E_{g}} \frac{1}{1+\frac{\epsilon-V+h_{8}-\frac{\hbar^{2} k^{2}}{2 m}}{E_{g}}}= \\
\frac{1}{E_{g}}\left\{1-\frac{\epsilon-V+h_{8}-\frac{\hbar^{2} k^{2}}{2 m}}{E_{g}}+\mathcal{O}\left(\frac{1}{E_{g}^{3}}\right)\right\},
\end{aligned}
$$

Podemos expandir a outra fração de forma análoga,

$$
\begin{aligned}
\frac{1}{\epsilon-V+h_{7}+E_{g}+\Delta_{g}-\frac{\hbar^{2} k^{2}}{2 m}}= & \frac{1}{E_{g}+\Delta_{g}} \frac{1}{1+\frac{\epsilon-V+h_{7}-\frac{\hbar^{2} k^{2}}{2 m}}{E_{g}+\Delta_{g}}}= \\
& \frac{1}{E_{g}+\Delta_{g}}\left\{1-\frac{\epsilon-V+h_{7}-\frac{\hbar^{2} k^{2}}{2 m}}{E_{g}+\Delta_{g}}+\mathcal{O}\left(\frac{1}{E_{g}^{3}}\right)\right\} .
\end{aligned}
$$

Podemos aplicar estas aproximações nas equações 2.4.9a e 2.4.9b para obtermos

$$
\begin{gathered}
\phi=\sqrt{\frac{2}{3}} \frac{P}{E_{g}}\left(1-\frac{\epsilon-V}{E_{g}}+\frac{\hbar^{2} k^{2}}{2 m E_{g}}\right) \boldsymbol{T}^{\dagger} \cdot \boldsymbol{k} \psi+\mathcal{O}\left(\frac{1}{E_{g}^{3}}\right) \\
\chi=-\frac{1}{\sqrt{3}} \frac{P}{E_{g}+\Delta_{g}}\left(1-\frac{\epsilon-V}{E_{g}+\Delta_{g}}+\frac{\hbar^{2} k^{2}}{2 m\left(E_{g}+\Delta_{g}\right)}\right) \boldsymbol{\sigma} \cdot \boldsymbol{k} \psi+\mathcal{O}\left(\frac{1}{E_{g}^{3}}\right),
\end{gathered}
$$

que determinam a banda de valência até segunda ordem em $1 / E_{g}$.

Alguém poderia levantar uma questão de fato muito séria: é lícito expandir uma hamiltoniana em Taylor e truncar em qualquer ordem sem problemas? Podemos mostrar que a hermiticidade é mantida de termo a termo em uma expansão de Taylor (veja o apêndice A.3), o que significa que o procedimento que faremos a seguir é completamente "seguro". 


\subsection{Equação efetiva para banda de condução}

Utilizando os resultados da seção 2.5 e as aproximações da seção 2.6 em conjunto com a equação 2.4.10, desenvolverei uma hamiltoniana efetiva para a banda de condução. Primeiramente, trabalharei um pouco melhor a equação 2.4.10. Usando as equações 2.6.3a e 2.6.3b, obtemos para $\psi$ a seguinte equação:

$$
\begin{aligned}
\frac{2 \mathcal{P}^{2}}{3 E_{g}} \boldsymbol{T} \cdot \boldsymbol{k}\left(1-\frac{\epsilon-V+h_{8}}{E_{g}}+\frac{\hbar^{2} k^{2}}{2 m E_{g}}\right) \boldsymbol{T}^{\dagger} \cdot \boldsymbol{k} \psi+ \\
\frac{\mathcal{P}^{2}}{3\left(E_{g}+\Delta_{g}\right)} \boldsymbol{\sigma} \cdot \boldsymbol{k}\left(1-\frac{\epsilon-V+h_{7}}{E_{g}+\Delta_{g}}+\frac{\hbar^{2} k^{2}}{2 m\left(E_{g}+\Delta_{g}\right)}\right) \boldsymbol{\sigma} \cdot \boldsymbol{k} \psi= \\
\quad\left(\epsilon-h_{6}-V-\frac{\hbar^{2} k^{2}}{2 m}\right) \psi
\end{aligned}
$$

Vou separar esta expressão enorme em duas contribuições que analisaremos separadamente,

$$
\begin{aligned}
\frac{2 \mathcal{P}^{2}}{3 E_{g}}\left[\boldsymbol{T} \cdot \boldsymbol{k}\left(1+\frac{\hbar^{2} k^{2}}{2 m E_{g}}\right) \boldsymbol{T}^{\dagger} \cdot \boldsymbol{k}-\boldsymbol{T} \cdot \boldsymbol{k} \frac{\epsilon-V+h_{8}}{E_{g}} \boldsymbol{T}^{\dagger} \cdot \boldsymbol{k}\right] \psi+ \\
\frac{\mathcal{P}^{2}}{3\left(E_{g}+\Delta_{g}\right)}\left[\boldsymbol{\sigma} \cdot \boldsymbol{k}\left(1+\frac{\hbar^{2} k^{2}}{2 m\left(E_{g}+\Delta_{g}\right)}\right) \boldsymbol{\sigma} \cdot \boldsymbol{k}-\boldsymbol{\sigma} \cdot \boldsymbol{k} \frac{\epsilon-V+h_{7}}{E_{g}+\Delta_{g}} \boldsymbol{\sigma} \cdot \boldsymbol{k}\right] \psi \\
=\left(\epsilon-h_{6}-V-\frac{\hbar^{2} k^{2}}{2 m}\right) \psi
\end{aligned}
$$

A equação 2.7.2 apresenta muitos e muitos termos. Vamos dividir para conquistar: como $\left[k_{j}, k_{p}\right]=0$, então os termos $\propto k_{z}^{2}$ podem ser facilmente trabalhados usando o resultado 2.5.10,

$$
\begin{array}{r}
\frac{\mathcal{P}^{2}}{3}\left[\boldsymbol{T} \cdot \boldsymbol{k} \frac{2}{E_{g}}\left(1+\frac{\hbar^{2} k^{2}}{2 m E_{g}}\right) \boldsymbol{T}^{\dagger} \cdot \boldsymbol{k}+\frac{1}{\left(E_{g}+\Delta_{g}\right)} \boldsymbol{\sigma} \cdot \boldsymbol{k}\left(1+\frac{\hbar^{2} k^{2}}{2 m\left(E_{g}+\Delta_{g}\right)}\right) \boldsymbol{\sigma} \cdot \boldsymbol{k}\right] \psi= \\
\frac{\mathcal{P}^{2}}{3}\left[\frac{2}{E_{g}}\left(1+\frac{\hbar^{2} k^{2}}{2 m E_{g}}\right) \boldsymbol{T} \cdot \boldsymbol{k} \boldsymbol{T}^{\dagger} \cdot \boldsymbol{k}+\frac{1}{\left(E_{g}+\Delta_{g}\right)}\left(1+\frac{\hbar^{2} k^{2}}{2 m\left(E_{g}+\Delta_{g}\right)}\right) \boldsymbol{\sigma} \cdot \boldsymbol{k} \boldsymbol{\sigma} \cdot \boldsymbol{k}\right] \psi= \\
\frac{\mathcal{P}^{2}}{3}\left[\frac{2}{E_{g}}\left(1+\frac{\hbar^{2} k^{2}}{2 m E_{g}}\right)+\frac{1}{\left(E_{g}+\Delta_{g}\right)}\left(1+\frac{\hbar^{2} k^{2}}{2 m\left(E_{g}+\Delta_{g}\right)}\right)\right] k^{2} \psi
\end{array}
$$

Assim, a equação efetiva para a banda de condução em primeira ordem de $1 / E_{g}$, de uma forma compacta, pode ser escrita como

$$
\frac{\mathcal{P}^{2}}{3}\left[\gamma_{1}+\gamma_{2} \frac{\hbar^{2} k^{2}}{2 m}\right] k^{2} \psi=(\epsilon-V) \psi,
$$

em que defini

$$
\gamma_{1}=\frac{2}{E_{g}}+\frac{1}{E_{g}+\Delta_{g}}
$$


e

$$
\gamma_{2}=\frac{2}{E_{g}^{2}}+\frac{1}{\left(E_{g}+\Delta_{g}\right)^{2}} .
$$

Estes dois fatores se mostram frequentes ao longo da dissertação toda (assim como em trabaIhos anteriores ${ }^{4}$ ). A equação 2.7.4 apresenta um termo proporcional a $P^{4}=\hbar^{4} k^{4}$, que pode ser interpretado como uma correção análoga à correção da massa relativística na equação de Dirac. Mais adiante, veremos outros termos (como o de Darwin e acoplamento spin-órbita) surgirem naturalmente, muito embora este termo proporcional a $P^{4}$ será cancelado.

Os próximos termos na equação 2.7.2 envolvem cálculos que já investigamos na seção 2.5. Vamos primeiramente estudar os termos com $\epsilon-V$ e depois, separadamente, os termos que envolvem o potencial estrutural $\left(h_{i}\right)$. Separei desta forma porque estas contribuições serão responsáveis por termos de natureza muito diferente. A equação 2.5 .13 mostra que

$$
\begin{aligned}
{\left[\frac{2}{E_{g}^{2}} \boldsymbol{T} \cdot \boldsymbol{k}(\epsilon-V) \boldsymbol{T}^{\dagger} \cdot \boldsymbol{k}+\right.} & \left.\frac{1}{\left(E_{g}+\Delta_{g}\right)^{2}} \boldsymbol{\sigma} \cdot \boldsymbol{k}(\epsilon-V) \boldsymbol{\sigma} \cdot \boldsymbol{k}\right] \psi= \\
& {\left[\gamma_{2} \epsilon k^{2}+2 \gamma_{2} V k^{2}+2 \gamma_{2}(\boldsymbol{k} V) \cdot \boldsymbol{k}-i \eta_{H} \boldsymbol{\sigma} \cdot(\boldsymbol{k} V) \times \boldsymbol{k}\right] \psi, }
\end{aligned}
$$

em que podemos identificar novamente 2.7 .6 e defini

$$
\eta_{H}=\frac{1}{E_{g}^{2}}-\frac{1}{\left(E_{g}+\Delta_{g}\right)^{2}} .
$$

Esta nomenclatura ficará clara mais tarde, mas podemos adiantar que este termo é responsável por parte do acoplamento spin-órbita.

Por fim, vamos tratar os termos que envolvem os perfis $h_{i}$, e utilizando exatamente os mesmos cálculos (para uma complementação, veja o apêndice A) podemos mostrar que

$$
\begin{array}{r}
\frac{2 \mathcal{P}^{2}}{3 E_{g}^{2}} \boldsymbol{T} \cdot \boldsymbol{k} h_{8} \boldsymbol{T}^{\dagger} \cdot \boldsymbol{k} \psi+\frac{\mathcal{P}^{2}}{3\left(E_{g}+\Delta_{g}\right)^{2}} \boldsymbol{\sigma} \cdot \boldsymbol{k} h_{7} \boldsymbol{\sigma} \cdot \boldsymbol{k} \psi= \\
\frac{\mathcal{P}^{2}}{3}\left[\gamma_{2}^{\prime} h_{w} k^{2}+\gamma_{2}^{\prime}\left(\boldsymbol{k} h_{w}\right) \cdot \boldsymbol{k}-i \eta_{e} \boldsymbol{\sigma} \cdot\left(\boldsymbol{k} h_{w}\right) \times \boldsymbol{k}\right] \psi,
\end{array}
$$

em que defini mais duas constantes análogas às definições 2.7.6 e 2.7.8:

$$
\gamma_{2}^{\prime}:=\frac{2 \delta_{8}}{E_{g}^{2}}+\frac{\delta_{7}}{\left(E_{g}+\Delta_{g}\right)^{2}}
$$

e

$$
\eta_{e}:=\frac{\delta_{8}}{E_{g}^{2}}-\frac{\delta_{7}}{\left(E_{g}+\Delta_{g}\right)^{2}} .
$$

Utilizar $\eta_{e}$ segue da mesma razão por que nomeamos $\eta_{H}$. Desta vez, estes fatores não dependem apenas de constantes dos materiais, mas também de parâmetros do poço $\left(\delta_{j}\right.$ são as 
alturas dos poços e barreiras, reveja a figura 2.4). A equação efetiva para a banda de condução $\psi$ se forma com a reunião das equações $2.7 .4,2.7 .7$ e 2.7.9.

Apesar de as equações para as bandas de condução e valência estarem desacopladas, ainda existe um vínculo, escondido de fato, que faz com que precisemos resolver as bandas de valência: a densidade de probabilidade. A densidade de probabilidade de encontrarmos o elétron em alguma posição do espaço é dada pelo produto $\Psi^{\dagger} \Psi$ calculado nesta posição. No entanto, $\Psi^{\dagger} \Psi=\psi^{\dagger} \psi+\phi^{\dagger} \phi+\chi^{\dagger} \chi$. Isso significa que $\psi$ fornece informações incompletas sobre o sistema, mesmo com sua equação estando desacoplada das bandas de valência. $\mathrm{O}$ que faremos a seguir é resolver este problema propondo um novo spinor de duas componentes, mas cuja densidade de probabilidade é a mesma que $\Psi$.

Escrever a equação efetiva neste momento é um tanto quanto desnecessário, pois veremos logo mais que muitos dos termos que calculamos serão cancelados na eliminação deste vínculo.

\subsubsection{Normalização apropriada: interpretação probabilística}

Gostaríamos de dar a $\psi$ característica de função de onda, isto é, gostaríamos que $|\psi|^{2}$ pudesse ser entendido como uma função densidade de probabilidade. A densidade de probabilidade de fato é

$$
|\Psi|^{2}=|\psi|^{2}+|\phi|^{2}+|\chi|^{2}
$$

Substituindo as equações 2.6.3a e 2.6.3b nesta expressão para $|\Psi|^{2}$, obtemos

$$
|\Psi|^{2}=|\psi|^{2}+\frac{2 \mathcal{P}^{2}}{3 E_{g}^{2}}\left|\boldsymbol{T}^{\dagger} \cdot \boldsymbol{k} \psi\right|^{2}+\frac{\mathcal{P}^{2}}{3\left(E_{g}+\Delta_{g}\right)^{2}}|\boldsymbol{\sigma} \cdot \boldsymbol{k} \psi|^{2}+\mathcal{O}\left(\frac{1}{E_{g}^{4}}\right) .
$$

Ou seja, a probabilidade de encontrarmos o elétron na região centrada em $\boldsymbol{r}$, cujo volume é $d^{3} r$, é descrita por $|\Psi(\boldsymbol{r})|^{2} d^{3} r$.

Suponha um novo spinor (de duas componentes) que satisfaça, quaisquer que sejam os domínios de integração,

$$
\int d^{3} r \psi_{S}^{\dagger} \psi_{S}=\int d^{3} r \Psi^{\dagger} \Psi
$$

Se este novo espinor realmente existir, então podemos pensar na possibilidade de expressá-lo como $\psi_{S}=L \psi$, de forma que

$$
\int d^{3} r \psi_{S}^{\dagger} \psi_{S}=\int d^{3} r\left(\psi^{\dagger} L^{\dagger}\right)(L \psi)=\int d^{3} r \Psi^{\dagger} \Psi
$$

Assim, $\psi_{S}=L \psi$ faz o papel da banda de condução renormalizada. Como os domínios de integrações na equação 2.7 .15 não importam, $\psi_{S}$ representa uma função de onda diferente 
mas que reproduz com exatidão a mesma densidade de probabilidade de $\Psi$. Note, então, que $\psi_{S}$ não é a função de onda do elétron, mas certamente é suficiente para calcularmos quaisquer observáveis com respeito à banda de condução. Usando a equação 2.7.13, podemos determinar $L$ explicitamente,

$$
\begin{gathered}
\int d^{3} r \psi_{S}^{\dagger} \psi_{S}=\int d^{3} r\left\{|\psi|^{2}+\frac{2 \mathcal{P}^{2}}{3 E_{g}^{2}}\left|\boldsymbol{T}^{\dagger} \cdot \boldsymbol{k} \psi\right|^{2}+\frac{\mathcal{P}^{2}}{3\left(E_{g}+\Delta_{g}\right)^{2}}|\boldsymbol{\sigma} \cdot \boldsymbol{k} \psi|^{2}\right\} \\
=\int d^{3} r\left\{|\psi|^{2}+\frac{2 \mathcal{P}^{2}}{3 E_{g}^{2}} \psi^{\dagger} \boldsymbol{T} \cdot \boldsymbol{k} \boldsymbol{T}^{\dagger} \cdot \boldsymbol{k} \psi+\frac{\mathcal{P}^{2}}{3\left(E_{g}+\Delta_{g}\right)^{2}} \psi^{\dagger} \boldsymbol{\sigma} \cdot \boldsymbol{k} \boldsymbol{\sigma} \cdot \boldsymbol{k} \psi\right\}
\end{gathered}
$$

Como as representações de $\boldsymbol{T} \cdot \boldsymbol{k} \boldsymbol{T}^{\dagger} \cdot \boldsymbol{k}$ e $\boldsymbol{\sigma} \cdot \boldsymbol{k} \boldsymbol{\sigma} \cdot \boldsymbol{k}$ são reais, podemos usar a representação 2.5.10 na equação 2.7.16 e simetrizá-las. Obtemos com isso

$$
\int d^{3} r \psi_{S}^{\dagger} \psi_{S}=\int d^{3} r\left\{\psi^{\dagger} \psi+\frac{2 \mathcal{P}^{2}}{3 E_{g}^{2}}\left(\frac{\psi^{\dagger} k^{2} \psi+\left(k^{2} \psi^{\dagger}\right) \psi}{2}\right)+\frac{\mathcal{P}^{2}}{3\left(E_{g}+\Delta_{g}\right)^{2}}\left(\frac{\psi^{\dagger} k^{2} \psi+\left(k^{2} \psi^{\dagger}\right) \psi}{2}\right)\right\}
$$

ou, em termos de $\gamma_{2}$ definido em 2.7.6,

$$
\int d^{3} r \psi_{S}^{\dagger} \psi_{S}=\int d^{3} r\left\{\psi^{\dagger} \psi+\frac{\mathcal{P}^{2}}{3} \gamma_{2}\left(\frac{\psi^{\dagger} k^{2} \psi+\left(k^{2} \psi^{\dagger}\right) \psi}{2}\right)\right\}
$$

A pergunta que queremos responder é "que espinor $\psi_{S}$ podemos definir para que 2.7.17 seja obedecida?" É até quase natural propor

$$
\psi_{S}=\left[1+\frac{\mathcal{P}^{2}}{6} \gamma_{2} k^{2}\right] \psi
$$

definindo assim a operação $L$. Podemos facilmente verificar que a equação 2.7.17 é satisfeita, pois

$$
\psi^{\dagger}\left[1+\frac{\mathcal{P}^{2}}{6} \gamma_{2} k^{2}\right] \times\left[1+\frac{\mathcal{P}^{2}}{6} \gamma_{2} k^{2}\right] \psi=\psi^{\dagger} \psi+\frac{\mathcal{P}^{2}}{3} \gamma_{2}\left(\frac{\psi^{\dagger} k^{2} \psi+\left(k^{2} \psi^{\dagger}\right) \psi}{2}\right)+\mathcal{O}\left(\frac{1}{E_{g}^{2}}\right) .
$$

Note por fim que $L$ é uma transformação não-unitária e por isso os módulos de $\psi$ e $\psi_{S}$ são diferentes.

Como gostaríamos de determinar $\psi_{S}$ em vez de $\psi$, seria mais interessante conhecer $\psi=$ $L^{-1} \psi_{S}$ e substitui-lo na equação 2.7.9. Para isso, basta inverter $L$ dado pela equação 2.7.18,

$$
\frac{1}{1+\frac{\mathcal{P}^{2}}{6} \gamma_{2} k^{2}}=1-\frac{\mathcal{P}^{2}}{6} \gamma_{2} k^{2}+\mathcal{O}\left(\frac{1}{E_{g}^{4}}\right) \text {. }
$$

Portanto, invertendo a equação 2.7.18, obtemos

$$
\psi=\left[1-\frac{\mathcal{P}^{2}}{6} \gamma_{2} k^{2}\right] \psi_{S},
$$


ou seja, $\psi$ é descrito em termos de $\psi_{S}$ mudando apenas um sinal.

\subsubsection{Hamiltoniana efetiva}

Vou a seguir derivar uma hamiltoniana efetiva para a banda de condução apropriadamente normalizada, $\psi_{S}=L \psi$. Ao somar as equações 2.7.4, 2.7.7 e 2.7.9, obtemos uma equação como

$$
\mathcal{H}_{s n} \psi=\left(\epsilon-h_{6}-V-\frac{\hbar^{2} k^{2}}{2 m}\right) \psi
$$

em que

$$
\begin{aligned}
\mathcal{H}_{s n}=\gamma_{2}^{\prime} h_{w} k^{2}+\gamma_{2}^{\prime}\left(\boldsymbol{k} h_{w}\right) \cdot \boldsymbol{k}+i \eta_{e} \boldsymbol{\sigma} \cdot\left(\boldsymbol{k} h_{w}\right) \times \boldsymbol{k}+\gamma_{2} \epsilon k^{2}+2 \gamma_{2} V k^{2}+ \\
\quad 2 \gamma_{2}(\boldsymbol{k} V) \cdot \boldsymbol{k}-\eta_{H} \boldsymbol{\sigma} \cdot(\boldsymbol{k} V) \times \boldsymbol{k}+\gamma_{1} k^{2}+\gamma_{2} \frac{\hbar^{2}}{2 m} k^{4}
\end{aligned}
$$

é a hamiltoniana efetiva "sem renormalização". Não fatorei diversos dos termos para explicitar cancelamentos que ocorrerão a seguir.

Como $\psi=L^{-1} \psi_{S}$, vou realizar o seguinte cálculo

$$
L^{-1} \mathcal{H}_{s n} L^{-1} \psi_{S}=L^{-1}\left(\epsilon-h_{6}-V-\frac{\hbar^{2} k^{2}}{2 m}\right) L^{-1} \psi_{S}
$$

Primeiramente, note que, usando a equação 2.7.20,

$$
L^{-1} \mathcal{H}_{s n} L^{-1}=\left[1-\frac{\mathcal{P}^{2}}{3} \gamma_{2} k^{2}\right] \mathcal{H}_{s n}\left[1-\frac{\mathcal{P}^{2}}{3} \gamma_{2} k^{2}\right]=\mathcal{H}_{s n}+\mathcal{O}\left(\frac{1}{E_{g}^{3}}\right)
$$

o que significa que o lado esquerdo da equação 2.7.23 não se altera. Vamos então analisar seu lado direito, que certamente gerará termos diferentes:

$$
\begin{aligned}
& {\left[1-\frac{\mathcal{P}^{2}}{3} \gamma_{2} k^{2}\right]\left(\epsilon-h_{6}-V-\frac{\hbar^{2} k^{2}}{2 m}\right)\left[1-\frac{\mathcal{P}^{2}}{3} \gamma_{2} k^{2}\right] \psi_{S}=} \\
& \left(\epsilon-h_{6}-V-\frac{\hbar^{2} k^{2}}{2 m}+\gamma_{2} \frac{\hbar^{2} k^{4}}{2 m}-2 \frac{\mathcal{P}^{2}}{6} \gamma_{2} \epsilon k^{2}+\right. \\
& \left.\frac{\mathcal{P}^{2}}{6} \gamma_{2}\left[2\left(V+h_{6}\right) k^{2}+\left(k^{2}\left[V+h_{6}\right]\right)+2\left(\boldsymbol{k}\left[V+h_{6}\right]\right) \cdot \boldsymbol{k}\right]\right) \psi_{S}+\mathcal{O}\left(\frac{1}{E_{g}^{4}}\right) .
\end{aligned}
$$

Se substituirmos 2.7.25 no lado direito da equação 2.7.23, podemos ver o cancelamento de diversos termos (basta comparar com a equação 2.7.22). Dentre os termos, vemos o cancelamento do termo $\mathcal{P}^{2} \gamma_{2} k^{2} \epsilon / 3$, o que aniquila qualquer dependência da hamiltoniana pela energia. Além disso, $\gamma_{2} \hbar^{2} k^{2} k_{z}^{2} / 2 m$ também se anula fantasticamente, não gerando nenhuma contribuição análoga à correção da massa relativística (que ocorre na equação de Dirac). 
Reunindo por fim todos os termos, obtemos (veja a seção A.1 para alguns detalhes adicionais)

$$
H_{\mathrm{eff}} \psi_{S}=\epsilon \psi_{S}
$$

com a hamiltoniana efetiva $H_{\text {eff }}$ dada por (mantendo todos os parâmetros explícitos)

$$
\begin{aligned}
H_{\mathrm{eff}}= & \frac{\hbar^{2}}{2 M} k^{2}+h_{6}+V-\frac{\mathcal{P}^{2}}{6}\left[\frac{2}{E_{g}^{2}}+\frac{1}{\left(E_{g}+\Delta_{g}\right)^{2}}\right] k^{2}\left(h_{6}+V\right) \\
& -\frac{\mathcal{P}^{2}}{3} i \boldsymbol{\sigma} \cdot\left\{\left[\frac{1}{E_{g}^{2}}-\frac{1}{\left(E_{g}+\Delta_{g}\right)^{2}}\right](\boldsymbol{k} V)-\left[\frac{\delta_{8}}{E_{g}^{2}}-\frac{\delta_{7}}{\left(E_{g}+\Delta_{g}\right)^{2}}\right]\left(\boldsymbol{k} h_{w}\right)\right\} \times \boldsymbol{k} \\
& -\frac{\mathcal{P}^{2}}{3}\left[\frac{2 \delta_{8}}{E_{g}^{2}}+\frac{\delta_{7}}{\left(E_{g}+\Delta_{g}\right)^{2}}+\gamma_{2} \delta_{6}\right]\left(\boldsymbol{k} h_{w}\right) \cdot \boldsymbol{k}
\end{aligned}
$$

Note que a energia cinética é dada em termos de uma massa efetiva $M$ que depende de $z$ implicitamente dada por,

$$
\frac{\hbar^{2}}{2 M}=\frac{\hbar^{2}}{2 m}+\frac{\mathcal{P}^{2}}{3}\left(\frac{2}{E_{g}}+\frac{1}{E_{g}+\Delta_{g}}\right)-\frac{\mathcal{P}^{2}}{3}\left[\frac{2 \delta_{8}}{E_{g}^{2}}+\frac{\delta_{7}}{\left(E_{g}+\Delta_{g}\right)^{2}}+\gamma_{2} \delta_{6}\right] h_{w} .
$$

No apêndice A.2 mostro uma outra forma de expressar esta massa efetiva. Esta massa $M$ depende da posição, tendo um pequeno salto dentro do poço - o que seria natural de se esperar: conforme o elétron passa de um material para outro, sua massa efetiva, numa visão de teoria de bandas $\|$, muda. Se $h_{w}$ for um potencial sem suavização, então a massa modifica-se abruptamente nas interface dos materiais, e mantém-se constante dentro dos mesmos materiais. Apesar de ser um pulo abrupto, devemos lembrar que o tamanho deste salto é pequeno e perceptível apenas em segunda ordem de $1 / E_{g}$.

É importante frisar que esta hamiltoniana efetiva difere da encontrada em outras referências ${ }^{1,3,4 * *}$. Isso porque, nestas referências, os autores preocuparam exclusivamente com o acoplamento spin-órbita. Por isso, nestas referências, os autores expandiram até segunda ordem em $1 / E_{g}$ apenas os termos não diagonais da equação efetiva para a banda de condução. Todas as correções que diferem daquelas referências são diagonais. Em suma, portanto, os trabalhos concordam perfeitamente. Mostrarei ainda adiante que as diferenças introduzidas neste trabalho podem ser incorporadas facilmente nestes outros trabalhos precedentes.

O termo proporcional a $k^{2}\left(h_{6}+V\right)$ é conhecido como termo de Darwin, já que lembra o o termo de Darwin da expansão não relativística da equação de Dirac. Um fato interessante

"Cada material, se isolado, tem sua própria estrutura de bandas. A massa efetiva em certo ponto da rede recíproca depende da curvatura da banda neste ponto.

**Principalmente porque este trabalho foi baseado na dissertação de mestrado de (e conversas particulares com) Rafael Calsaverini ${ }^{1}$ (além dos artigos citados no texto). 
é que esta correção leva em conta apenas $h_{6}$ e $V$, i.e., é uma contribuição exclusivamente da banda de condução, deixando de lado os perfis experimentados pelas bandas de valência. $O$ termo proporcional ao produto $\boldsymbol{k} V \cdot \boldsymbol{k}$, como comentado mais cedo, é um tipo de acoplamento entre o campo elétrico externo e o momento linear.

Apesar de parecer que este resultado não tem nada a ver mais com a equação de Dirac, principalmente por causa do acoplamento momento - campo elétrico, no apêndice $\mathrm{B}$ discuto o porquê dessas diferenças e mostramos que mesmo este termo emerge da equação de Dirac se considerarmos potenciais diferentes para as partículas e para as antipartículas.

Se compararmos com famoso proceedings de Roland Winkler ${ }^{41}$, podemos ver algumas aparentes diferenças, discutidas no apêndice C. As equações não são exatamente as mesmas - a menos talvez de um problema de digitação -, muito embora os métodos sejam diferentes.

\subsubsection{Finalizando: representação em $\langle r|$}

Vou, a seguir, calcular a representação de 2.7 .27 na base do operador $\boldsymbol{R},\langle\boldsymbol{r}|=\langle x y z|$. Nesta base,

$$
\langle\boldsymbol{r}| V(Z)=V(z)\langle\boldsymbol{r}| \quad \text { e } \quad\langle\boldsymbol{r}| \boldsymbol{k}=-i \boldsymbol{\nabla}\langle\boldsymbol{r}|
$$

Quando aplicamos estes operadores a uma função que depende exclusivamente de $z$, representaremos a ação como

$$
(\boldsymbol{k} V) \rightarrow-i \boldsymbol{\nabla} V(z)=\left(0,0,-i \frac{d V}{d z}(z)\right)
$$

Alguns dos operadores $k$ não atuam sobre os potenciais (estrutural ou de interação), mas sim sobre a função de onda. Neste caso, como a base é $e^{i \boldsymbol{k}_{\|} \cdot \boldsymbol{r}} f(z)$, então como resultado temos $k_{x}$ e $k_{y}$ como números e $k_{z} \mapsto-i d / d z$. Veremos, logo mais, que apenas um único termo (o da energia cinética) fornecerá uma contribuição com todas as componentes de $\boldsymbol{k}$ sobre a função de onda. Portanto, a representação correta para o acoplamento momento-campo elétrico é

$$
\left(\boldsymbol{k} h_{w}\right) \cdot \boldsymbol{k} \rightarrow-\frac{d h_{w}}{d z}(z) \frac{d}{d z}
$$

e para o termo de Darwin é

$$
k^{2}\left(h_{6}+V\right) \rightarrow-\nabla^{2}\left(h_{6}(z)+V(z)\right)=-\frac{d^{2} h_{6}}{d z^{2}}(z)-\frac{d^{2} V}{d z^{2}}(z) .
$$

Talvez o mais curioso seja o caso do acoplamento spin-órbita: a parte da equação efetiva 2.7.27 proporcional a $\boldsymbol{\sigma}$. Chamaremos este termo de $H_{s o}$. Os coeficientes, contribuintes do termo de acoplamento spin-órbita, provém de duas fontes distintas: do potencial de interação (veja 
a a equação 2.7.8), e do potencial estrutural (veja equação 2.7.11). Desta forma, podemos escrever a contribuição deste acoplamento como

$$
H_{s o}:=-i \frac{\mathcal{P}^{2}}{3} \boldsymbol{\sigma} \cdot\left[\eta_{H} \boldsymbol{k} V-\eta_{e} \boldsymbol{k} h_{w}\right] \times \boldsymbol{k} .
$$

Note no entanto que

$$
\eta_{H} \boldsymbol{k} V-\eta_{e} \boldsymbol{k} h_{w} \rightarrow-i\left(0,0, \eta_{H} \frac{d V}{d z}(z)-\eta_{e} \frac{d h_{w}}{d z}(z)\right)
$$

Usando a ciclicidade do produto triplo,

$$
\boldsymbol{\sigma} \cdot\left[\eta_{H} \boldsymbol{k} V-\eta_{e} \boldsymbol{k} h_{w}\right] \times \boldsymbol{k}=\left[\eta_{H} \boldsymbol{k} V-\eta_{e} \boldsymbol{k} h_{w}\right] \cdot \boldsymbol{k} \times \boldsymbol{\sigma} .
$$

Como, de 2.7.34, $\left[\eta_{H} \boldsymbol{k} V-\eta_{e} \boldsymbol{k} h_{w}\right]$ tem apenas componentes em $\hat{z}$, então

$$
\hat{z} \cdot \boldsymbol{k} \times \boldsymbol{\sigma}=\hat{z} \cdot\left(k_{y} \sigma_{z}-k_{z} \sigma_{y}, k_{z} \sigma_{x}-k_{x} \sigma_{z}, k_{x} \sigma_{y}-k_{y} \sigma_{x}\right) .
$$

Assim a contribuição 2.7.33 finalmente toma a forma

$$
H_{s o}=-\eta(z)\left\{k_{x} \sigma_{y}-k_{y} \sigma_{x}\right\}
$$

que é a forma usual que gera o acoplamento inter e intrasub-bandas ${ }^{4}$. Para tal definimos a função

$$
\eta(z)=\frac{\mathcal{P}^{2}}{3}\left\{\eta_{H} \frac{d V}{d z}(z)-\eta_{e} \frac{d h_{w}}{d z}(z)\right\},
$$

que dará origem ao que chamamos de acoplamentos inter e intrasub-bandas. A parte matricial da equação 2.7.37 pode ser escrita explicitamente como

$$
k_{x} \sigma_{y}-k_{y} \sigma_{x}=\left(\begin{array}{cc}
0 & -i k_{-} \\
i k_{+} & 0
\end{array}\right)
$$

Tratarei a diagonalização deste termo, que é o único não diagonal na atual base de spin, na seção 3.2 .

Desta forma, a hamiltoniana efetiva, escrita na representação $\langle\boldsymbol{r}|$, pode ser escrita como

$$
\begin{aligned}
H_{\mathrm{eff}}=-\frac{\hbar^{2}}{2 M} \nabla^{2}+h_{6}+V+ & \frac{\mathcal{P}^{2}}{6} \gamma_{2}\left\{\frac{d^{2} h_{6}}{d z^{2}}(z)+\frac{d^{2} V}{d z^{2}}(z)\right\} \\
& -\frac{\mathcal{P}^{2}}{3}\left[\gamma_{2}^{\prime}+\gamma_{2} \delta_{6}\right] \frac{d h_{w}}{d z}(z) \frac{d}{d z}-\eta(z)\left[k_{x} \sigma_{y}-k_{y} \sigma_{x}\right] .
\end{aligned}
$$

Esta hamiltoniana será central para o que segue neste trabalho. No capítulo seguinte, mostrarei uma solução semianalítica de 2.7.40 que leva em conta numericamente a interação entre 
elétrons dentro da aproximação de Hartree. No capítulo 4, vou derivar uma nova equação efetiva que conta com a presença dos campos elétrico e magnético. Vou, então, modelar a interação elétron-fóton dentro da banda de condução. Tanto o termo de Darwin, como o termo proporcional a $\boldsymbol{k} h_{w} \cdot \boldsymbol{k}$ (sem analogia com a equação de Dirac) serão tratados analiticamente na seção 3.4 .

\subsection{Objeção de Ando}

Darr, Kotthays e Ando ${ }^{\dagger \dagger}$ (1977 apud Calsaverini $2007^{1}$ ) publicaram uma objeção muito interessante quanto à existência do acoplamento spin-órbita para a banda de condução. Atualmente, esta objeção leva o nome de "Objeção de Ando". A priori, a equação 2.7.27 mostra claramente que existe um acoplamento spin-órbita, no entanto a objeção de Ando traz à tona um detalhe que gostaria de comentar.

Uma explicação comum para o acoplamento de Rashba, e de fato um tanto quanto imprevista, apresenta a equação 1.2.1 como acoplamento spin-órbita para a banda de condução, utilizando o potencial $V(\boldsymbol{R})$ como o potencial confinador (ou, especificamente, o poço $h_{6}$ ). Como vemos acima, a diferença $\Delta E$ na energia gerado pelo acoplamento de Rashba pode ser medido usando um estado qualquer $|\psi\rangle$ como

$$
\Delta E \propto\left\langle\psi\left|\frac{d V}{d z}\right| \psi\right\rangle
$$

No entanto, para qualquer estado ligado o valor esperado da força $\frac{d V}{d z}$ generalizada é nulo, se $V$ for o potencial confinador (ou ligante). Portanto, $\Delta E$ deveria ser nulo.

No entanto, como vimos ao longo deste capítulo, o acoplamento spin-órbita não é gerado por $h_{6}$, mas sim dos perfis de potencial das bandas de valência, que não são os potenciais confinadores. É sempre importante citar esta objeção para valorizar ainda mais a inexistência de $h_{6}$ no termo de acoplamento spin-órbita da equação 2.7 .27 , fato que aliás pode passar despercebido. Atualmente, podemos ver este problema discutido em algumas referências 1,73,74. O acoplamento de Rashba é um acoplamento que ocorre quando o poço admite apenas um estado ligado, o que chamamos de sub-banda. Note que esta argumentação não vale apenas para o acoplamento intrasub-bandas (Rashba), mas também vale para o acoplamento intersubbandas (caso em que o poço tem mais de uma sub-banda, que mostraremos no capítulo 3).

†'DARR, A.; KOTTHAYS, J.; ANDO, T. In: FUMI, F. G. (Ed.). In: INTERNATIONAL CONFERENCE ON THE PHYSICS OF SEMICONDUCTORS, 13., Rome, Italy, 1976. Amsterdã: North Holland, 1977. p. 774-778. 


\subsection{Revisão}

Deduzi identidades algébricas para os operadres que aparecem na hamiltoniana,

$$
T_{x}^{\dagger}=\frac{1}{2}\left[\begin{array}{cc}
-\sqrt{3} & 0 \\
0 & -1 \\
1 & 0 \\
0 & \sqrt{3}
\end{array}\right], \quad T_{y}^{\dagger}=\frac{i}{2}\left[\begin{array}{cc}
\sqrt{3} & 0 \\
0 & 1 \\
-1 & 0 \\
0 & \sqrt{3}
\end{array}\right], \quad T_{z}^{\dagger}=\left[\begin{array}{ll}
0 & 0 \\
1 & 0 \\
0 & 1 \\
0 & 0
\end{array}\right] .
$$

As regras podem ser todas sintetizadas na equação

$$
\boldsymbol{T} \cdot \boldsymbol{A T} \cdot \boldsymbol{B}=\mathbb{1}_{2 \times 2} \boldsymbol{A} \cdot \boldsymbol{B}-\frac{i}{2} \boldsymbol{\sigma} \cdot \boldsymbol{A} \times \boldsymbol{B} .
$$

De posse desta identidade, deduz a equação efetiva para a banda de condução:

$$
H_{\mathrm{eff}}=\frac{\hbar^{2}}{2 M} k^{2}+h_{6}+V+V_{\text {so }}+V_{d}
$$

em que defini

$$
\begin{aligned}
\frac{\hbar^{2}}{2 M} & =\frac{\hbar^{2}}{2 m}+\frac{\mathcal{P}^{2}}{3} \gamma_{1}+\frac{\mathcal{P}^{2}}{3}\left[\gamma_{2}^{\prime}+\gamma_{2} \delta_{6}\right] h_{w}, \\
V_{d} & =-\frac{\mathcal{P}^{2}}{6} \gamma_{2} k^{2}\left(h_{6}+V\right)-\frac{\mathcal{P}^{2}}{3}\left[\gamma_{2}^{\prime}+\gamma_{2} \delta_{6}\right]\left(\boldsymbol{k} h_{w}\right) \cdot \boldsymbol{k}, \\
V_{s o} & =-\frac{\mathcal{P}^{2}}{3} i \boldsymbol{\sigma} \cdot\left\{\eta_{H}(\boldsymbol{k} V)-\eta_{e}\left(\boldsymbol{k} h_{w}\right)\right\} \times \boldsymbol{k} .
\end{aligned}
$$

Defini as constantes

$$
\begin{aligned}
\gamma_{1} & =\frac{2}{E_{g}}+\frac{1}{E_{g}+\Delta_{g}}, \\
\gamma_{2} & =\frac{2}{E_{g}^{2}}+\frac{1}{\left(E_{g}+\Delta_{g}\right)^{2}}, \\
\gamma_{2}^{\prime} & =\frac{2 \delta_{8}}{E_{g}^{2}}+\frac{\delta_{7}}{\left(E_{g}+\Delta_{g}\right)^{2}}, \\
\eta_{H} & =\frac{1}{E_{g}^{2}}-\frac{1}{\left(E_{g}+\Delta_{g}\right)^{2}}, \\
\eta_{e} & =\frac{\delta_{8}}{E_{g}^{2}}-\frac{\delta_{7}}{\left(E_{g}+\Delta_{g}\right)^{2}} .
\end{aligned}
$$




\section{CAPÍTULO 3}

\section{Solução para a Banda de Condução}

Rock is not the devils work, it's magical and rad I'll never rock as long as I am stuck here with my dad Jables, em Tenacious D - Kickapoo, 2007.

Neste capítulo, eu gostaria de dedicar nossa atenção às soluções da equação efetiva deduzida no capítulo 2 para o caso de duas sub-bandas. Separarei as contribuições de ordem zero em $1 / E_{g}$ e as resolveremos autoconsistentemente. Além disso, comentarei brevemente sobre as soluções para uma sub-banda e sobre um tema atual e muito interessante: a interpretação do acoplamento spin-órbita como um campo magnético interno.

\subsection{Soluções semianalíticas para a equação efetiva}

Vamos reescrever a hamiltoniana dada em 2.7.40 como

$$
H_{\mathrm{eff}}=\left[\frac{\hbar^{2} k^{2}}{2 m}+h_{6}+V+\alpha_{D} k^{2}\left(\delta_{6} h_{w}+V\right)+\alpha_{s}\left(k_{z} h_{w}\right) \cdot k_{z}\right] \mathbb{1}_{2 x 2}-\eta(z)\left[k_{x} \sigma_{y}-k_{y} \sigma_{x}\right],
$$

em que defino as constantes

$$
\begin{aligned}
\alpha_{D} & :=-\frac{\mathcal{P}^{2}}{6}\left[\frac{2}{E_{g}^{2}}+\frac{1}{\left(E_{g}+\Delta_{g}\right)^{2}}\right], \\
\alpha_{M} & :=-\frac{\mathcal{P}^{2}}{3}\left[\gamma_{2}^{\prime}+\gamma_{2} \delta_{6}\right]
\end{aligned}
$$

e utilizei a definição de $\eta(z)$ da equação 2.7.38. Nesta hamiltoniana efetiva, existem termos de ordem zero em $1 / E_{g}$ e termos de segunda ordem. Por questões práticas, vou também aproximar a massa efetiva, utilizando apenas sua contribuição de primeira ordem em $1 / E_{g}$, 
i.e.,

$$
\frac{\hbar^{2}}{2 M}=\frac{\hbar^{2}}{2 m}+\frac{2}{E_{g}}+\frac{1}{E_{g}+\Delta_{g}}+\mathcal{O}\left(\frac{1}{E_{g}^{2}}\right) .
$$

Assim elimino a dependência em $z$ da massa efetiva, o que deve gerar efeitos desprezíveis para as soluções. A hamiltoniana $H_{\text {eff }}$ pode ser dividida em duas partes usando como discernimento a ordem em $1 / E_{g}$ : trataremos numericamente a primeira ordem,

$$
H_{\mathcal{N}}=-\frac{\hbar^{2}}{2 M} k^{2}+h_{6}+V(z),
$$

que é diagonal no spin e certamente contém os termos mais expressivos; e analiticamente os termos de segunda ordem,

$$
H_{\mathcal{A}}=\alpha_{s}\left(k_{z} h_{w}\right) \cdot k_{z}+\alpha_{D} k^{2}\left(h_{6}+V\right)+\eta(z)\left[k_{x} \sigma_{y}-k_{y} \sigma_{x}\right] .
$$

Como os termos de ordem zero são mais expressivos, utilizarei os resultados obtidos de $H_{\mathcal{N}}$ como ponto de partida. Por esse motivo chamamos este tratamento de semianalítico.

A hamiltoniana $H_{\mathcal{N}}$ é praticamente uma hamiltoniana usual de poços quânticos ${ }^{7}$. A única diferença com o que conhecemos dos livros textos é o potencial de interação $V$, que usarei como a interação elétron-elétron de Coulomb. Usarei, por simplicidade, a aproximação de Hartree, que leva em conta apenas o termo de correlação da interação de Coulomb ${ }^{1}$. O perfil de potencial $h_{6}$ dita, por exemplo, o número de estados ligados aceitos neste poço. No entanto, $h_{6}$ não é escolhido arbitrariamente: depende da concentração de doadores na região central, gap dos materiais, split off, etc. Existe um estudo gigantesco que resume informações sobre os materiais que constituem a estrutura a ser analisada em alguns poucos parâmetros (potencial químico, potencial estrutural, massa efetiva, etc.). Na literatura, podemos encontrar estes parâmetros para inúmeras estruturas, como no review de Vurgaftman et al ${ }^{70}$. Vou me restringir ao caso em que os parâmetros são tais que só há dois estados ligados. Cada estado ligado do poço quântico chamaremos de sub-bandas.

\subsubsection{Sobre $H_{\mathcal{N}}$ e separação de variáveis}

Notavelmente, a direção de crescimento do poço (a saber, $z$ ) é privilegiada pela hamiltoniana $H_{\mathcal{N}}$. A única operação em $x$ e $y$ de $H_{\mathcal{N}}$ fica no termo cinético, i.e.,

$$
H_{\mathcal{N}}=-\frac{\hbar^{2}}{2 M} k_{\|}^{2}-\frac{\hbar^{2}}{2 M} k_{z}^{2}+h_{6}+V(z),
$$


em que $\boldsymbol{k}_{\|}$é a projeção $\boldsymbol{k}$ no plano paralelo às interfaces, $\boldsymbol{k}_{\|}=\left(k_{x}, k_{y}\right)$. Chamando a solução desta hamiltoniana de $f(\boldsymbol{r})$, então podemos separá-la automaticamente no produto

$$
f(\boldsymbol{r})=e^{\boldsymbol{k}_{\|} \cdot \boldsymbol{r}} u(z) .
$$

Neste caso, $u(z)$ é a solução da parte em $z$ e a exponencial $e^{\boldsymbol{k}_{\|} \cdot \boldsymbol{r}}$ é uma das soluções possíveis para a parte trivial dependente de $x$ e $y$. Das condições de contorno aplicadas a $f(\boldsymbol{r})$, ficam definidos os possíveis valores de $k_{x}$ e $k_{y}$. Por fim, $u(z)$ deve ser uma função localizada na região central do poço, o que indica o confinamento do elétron*.

Ao escrever esta solução, estou admitindo que os elétrons estão confinados numa região da direção $z$, mas comportam-se como elétrons livres no plano $x-y$. Dizemos então que estes elétrons formam um gás bidimensional (como comentei no capítulo 1, um 2DEG). Este 2DEG tem seu espectro discretizado por condições de contorno em $x$ e $y$ que podem ser aplicadas sobre as funções de onda: origina-se o espaço recíproco (bidimensional) deste gás. Cada ponto deste espaço recíproco é um estado eletrônico possível.

No que segue, chamo de $u_{0}$ e $u_{1}$ as duas primeiras soluções de $H_{\mathcal{N}}$ obtidas pelos cálculos numéricos, e respectivamente suas energias $\epsilon_{0}$ e $\epsilon_{1}$. Como estas duas funções são linearmente independentes $^{\dagger}$, podemos criar a base $\left\{\left|u_{0} \uparrow\right\rangle,\left|u_{0} \downarrow\right\rangle,\left|u_{1} \uparrow\right\rangle,\left|u_{1} \downarrow\right\rangle\right\}$, em que

$$
\left|u_{j} \mu\right\rangle:=\left|u_{j}\right\rangle \otimes|\mu\rangle
$$

com os estados $|\mu\rangle$ sendo os estados de spin autovetores de $\sigma_{z}$, e definimos

$$
\left|u_{j}\right\rangle:=\int d z u_{j}(z)|z\rangle
$$

Para um operador qualquer $\hat{K}=(\hat{F} \otimes \mathbb{1})(\mathbb{1} \otimes \hat{G})$, ou seja, $\hat{F}$ só opera no subespaço da base de $\boldsymbol{R}$ e $\hat{G}$ só opera no subespaço de spin, vale

$$
\left\langle u_{j} \mu|\hat{K}| u_{p} \nu\right\rangle=\left\langle u_{j}|\hat{F}| u_{p}\right\rangle\langle\mu|\hat{G}| \nu\rangle
$$

A seguir, identifico os operadores $\hat{K}$ presentes na hamiltoniana e calculo seus elementos de matriz nesta base recém introduzida.

*Soluções não confinadas são possibilidades também, mas não nos interessam.

${ }^{\dagger}$ Autofunções com autovalores diferentes de um operador hermitiano. 


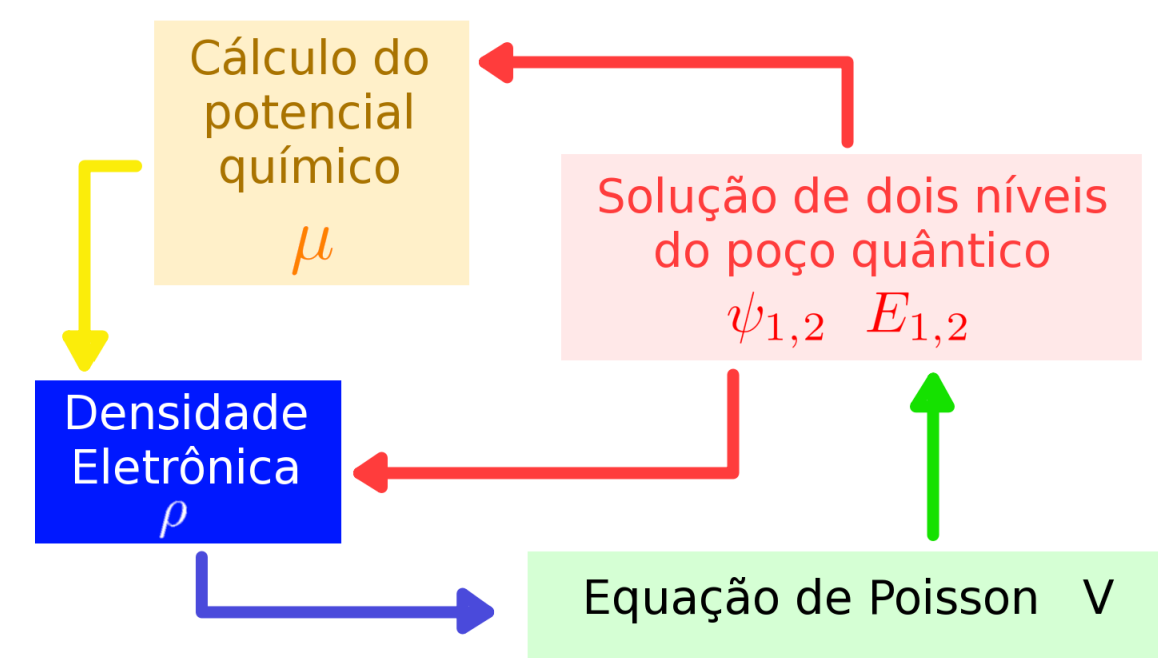

Figura 3.1 - A autoconsistência é gerada pela necessidade de se conhecer a distribuição eletrônica $\rho$, dada pelas soluções $\psi_{1,2}$ e $E_{1,2}$, e o potencial químico $\mu$ para calcular o potencial eletrônico $V$. A condição inicial é $V=0$, a partir da qual calculamos $\psi_{1,2}$ e $E_{1,2}$. Calculamos com isso o potencial químico $\mu$ e uma densidade $\rho$ preliminar. Assim, podemos resolver a equação de Poisson para determinar $V$ e podemos também recalcular $\mu$. Com este novo potencial $V$, podemos calcular novas soluções $\psi_{1,2}$ e $E_{1,2}$. Com isso fica evidente a autoconsistência entre as variáveis. Os sentidos das flechas indicam "quem fornece informação para quem".

\subsubsection{Cálculos numéricos}

As soluções de $\mathcal{H}_{\mathcal{N}}$ dependem do potencial $V$ de interação entre elétrons. Por outro lado, a equação de Poisson fornece este potencial somente se conhecermos a densidade de cargas, $\rho(\boldsymbol{r})$, em todo o espaço. Já a densidade depende das funções de onda resultantes de $\mathcal{H}_{\mathcal{N}}$ e do potencial químico. Claramente, estas variáveis estão ligadas num círculo vicioso, que chamamos de autoconsistência: para conhecermos o potencial de interação precisamos conhecer as posições dos elétrons (ou ao menos sua distribuição de probabilidades), e para conhecermos as posições precisamos conhecer o potencial de interação. A figura 3.1 resume estas informações e as relações de dependências entre estas variáveis.

Meu algoritmo realiza os seguintes passos: $(i)$ imponho $V=0$ e resolvo os dois primeiros níveis (autovalores e suas respectivas autofunções) de $\mathcal{H}_{\mathcal{N}}$; (ii) utilizando estas soluções, calculo o potencial químico; (iii) as soluções de $\mathcal{H}_{\mathcal{N}}$ e o potencial químico fornecem a densidade de carga; (iv) incluo a densidade na equação de Poisson para obtermos o potencial de interação; ( $v$ ) volto a resolver $\mathcal{H}_{\mathcal{N}}$ com a nova estimativa de $V$. Repetindo o ciclo $i \rightarrow i i \rightarrow i i i \rightarrow i v \rightarrow$ $v$, analiso as diferenças, entre uma iteração e outra, nas energias e nas funções de onda. O 
algoritmo interrompe-se quando estas diferenças são imperceptíveis em precisão dupla $a^{\ddagger}$. Este mesmo procedimento foi implementado em detalhes na dissertação de Rafael Calsaverini ${ }^{1}$ e os resultados destes cálculos foram exaustivamente explorados para diferentes materiais.

Desenvolvi um programa, envolvendo rotinas de Fortran 90, Maple e scripts Shell, que resolve os dois primeiros níveis de $H_{\mathcal{N}}$ e suas funções de onda usando o procedimento autoconsistente que acabo de detalhar. Para resolver a equação diferencial $H_{\mathcal{N}} u_{j}(z)=\epsilon_{j} u_{j}(z)$ utilizo o método Numerov de sexta ordem ${ }^{75}$ baseado na implementação algébrica de Bernardes ${ }^{76}$. A equação de Poisson foi resolvida utilizando um método Numerov adaptado também de sexta ordem $^{77,78}$.

Na figura 3.2 mostro algumas soluções de $u_{0}$ e $u_{1}$ para um poço formado por $A l \operatorname{In} S b / \operatorname{In} S b$. Tendo uma idéia melhor do como são as funções de base, podemos progredir com mais confiança. Esta estrutura possui o maior $\Delta_{g}$ e o menor $E_{g}$ entre as ligas binárias semicondutoras do grupo $I I I-V^{70}$, e por isso seu acoplamento spin-órbita deve ser o mais intenso ${ }^{4}$.

\subsubsection{Adição de barreiras}

A adição de barreiras não será tratada nas seções seguintes por ser trivial a generalização. Os três perfis de potencial com que trabalhamos são $h_{6}$ (banda de condução), $h_{7}$ e $h_{8}$ (ambos das bandas de valência). Estes três potenciais são descritos unificadamente usando o perfil unitário $h_{w}$, da equação 2.4 .2 , como

$$
h_{j}(z)=\delta_{j} h_{w}(z)
$$

Assim, para os nossos materiais, $\delta_{6}>0$ e $\delta_{7}, \delta_{8}<0$. Para adicionarmos barreiras, podemos redefinir estes potenciais da seguinte forma:

$$
h_{j}(z)=\delta_{j} h_{w}(z)+\delta_{j}^{b} h_{w}^{b}(z),
$$

em que $\delta_{j}^{b}$ são as alturas das barreiras e

$$
h_{w}^{b}(z):=-1+\mathfrak{H}\left(z+a_{b}\right)-\mathfrak{H}\left(z-a_{b}\right) .
$$

Neste perfil $h_{w}^{b}$ de barreira usamos $a_{b}$ como sendo a posição em que a barreira começa, análogo ao parâmetro $a_{0}$. Podemos ver na figura 3.2(d) o que ocorre quando adicionamos barreiras ao poço. Isto equivale a adicionar mais camadas de materiais diferentes.

Todos os cálculos que seguem neste capítulo podem ser estendidos para o caso com

$\ddagger$ Oito casas decimais em ponto flutuante. 


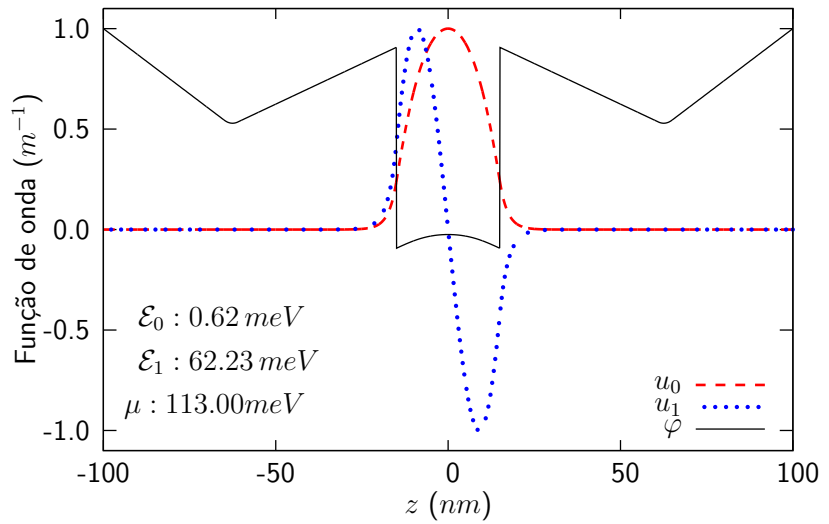

(a) $A l_{0.4} I n_{0.6} S b$ simétrico

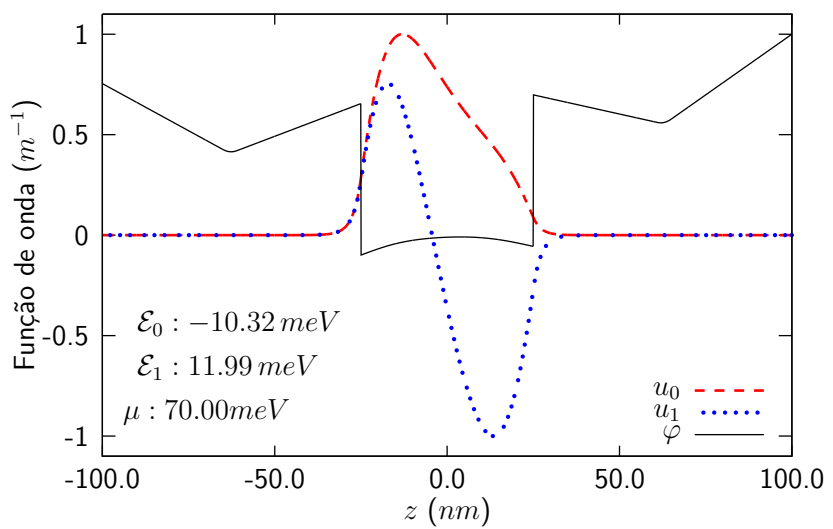

(c) $A l_{0.4} I n_{0.6} S b$ assimétrico

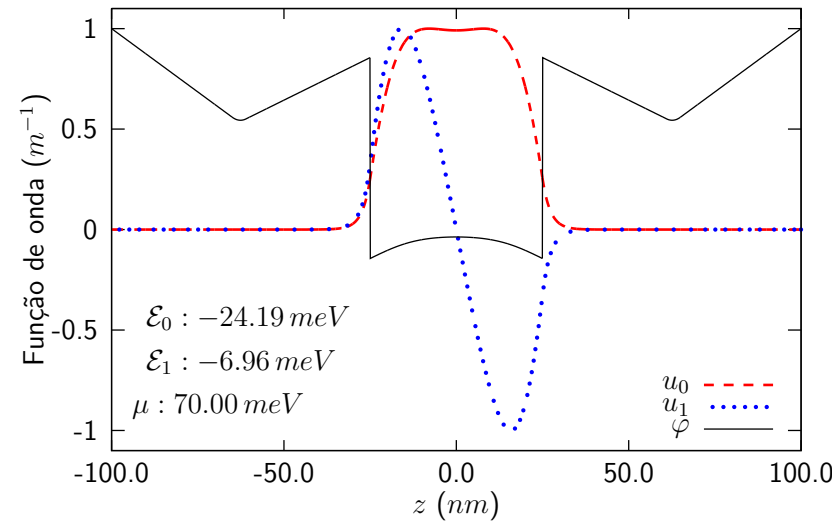

(b) $\mathrm{Al}_{0.4} \operatorname{In}_{0.6} \mathrm{Sb}$ simétrico com poço largo

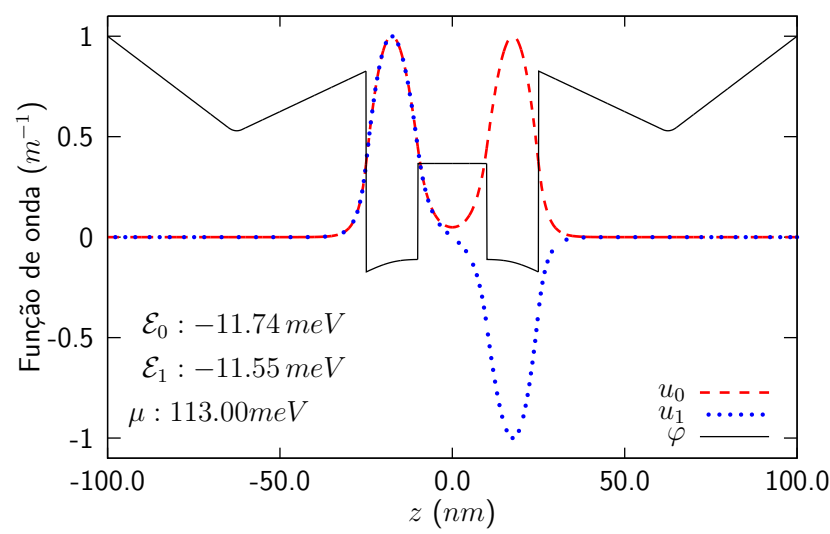

(d) $\mathrm{Al}_{0.4} I n_{0.6} \mathrm{Sb}$ com barreira

Figura 3.2 - Soluções desta abordagem para as bandas de condução para a estrutura $A l_{0.4} I n_{0.6} S b$. Mantive o potencial químico fixo em todas estas simulações, com a densidade de doadores de $3 \times 10^{18} \mathrm{~cm}^{-2}$ e à temperatura $0.3 \mathrm{~K}$. A região dos dopantes tem largura fixa de $4 \mathrm{~nm}$. 3(b) Dois níveis para um poço de $30 \mathrm{~nm}$ de largura e potencial químico fixo em $113 \mathrm{meV}$; 3(a) os mesmos dois níveis para um poço de $50 \mathrm{~nm}$ e potencial químico fixo em $70 \mathrm{meV}$.

múltiplas barreiras de forma trivial.

\subsection{Diagonalização do acoplamento spin-órbita e repre- sentação matricial}

O operador $k_{x} \sigma_{y}-k_{y} \sigma_{x}$ é o único termo não diagonal (em spin) na hamiltoniana 3.1.1. A base deste operador certamente mantém os outros operadores da hamiltoniana diagonais. Esta base, portanto, desacopla as componentes de spin, o que ajudaria imensamente a obter as autoenergias e as funções de onda do poço quântico. Na forma matricial, usando a base 
de $\sigma_{z}$, este operador fica

$$
k_{x} \sigma_{y}-k_{y} \sigma_{x}=\left(\begin{array}{cc}
0 & -i k_{-} \\
i k_{+} & 0
\end{array}\right) .
$$

Diagonalizar a matriz dada em 3.2.1 é simples: a matriz de transformação é

$$
\mathcal{U}:=\frac{1}{\sqrt{2}}\left(\begin{array}{cc}
i e^{i \theta} & 1 \\
-i e^{i \theta} & 1
\end{array}\right)
$$

e os autovetores são

$$
| \pm\rangle=\frac{\mp i e^{-i \theta}|\uparrow\rangle+|\downarrow\rangle}{\sqrt{2}} .
$$

Cada autovetor $| \pm\rangle$ está ligado ao autovalor \pm 1 . Diagonalizando o termo de spin-órbita,

$$
\mathcal{U}\left(k_{x} \sigma_{y}-k_{y} \sigma_{x}\right) \mathcal{U}^{-1}=\left(\begin{array}{cc}
k_{\|} & 0 \\
0 & -k_{\text {॥ }}
\end{array}\right) .
$$

Se definirmos $\left|u_{j} \pm\right\rangle:=\left|u_{j}\right\rangle \otimes| \pm\rangle$, é extremamente natural pensar na base

$$
\left\{\left|u_{0}+\right\rangle,\left|u_{1}+\right\rangle,\left|u_{0}-\right\rangle,\left|u_{1}-\right\rangle\right\}
$$

formada pelas autofunções de $H_{\mathcal{N}}$ e pelos autovetores de $k_{x} \sigma_{y}-k_{y} \sigma_{x}$. A ordem em que escrevi a base serve para diagonalizar por blocos a hamiltoniana efetiva, como ficará explícito já na seção seguinte.

Fora o acoplamento spin-órbita, os outros termos são todos diagonais. Portanto, são todos invariantes segundo $\mathcal{U} \cdot \mathcal{U}^{-1}$. A representação matricial de $H_{\mathcal{N}}$, cujas soluções chamamos de $u_{0}$ e $u_{1}$ e seus respectivos autovalores de $\epsilon_{0}$ e $\epsilon_{1}$, nesta base é trivial:

$$
\left\langle u_{j} \mu\left|H_{\mathcal{N}}\right| u_{p} \nu\right\rangle=\delta_{j, p} \delta_{\mu, \nu} \epsilon_{j}
$$

Assim, a matriz $H_{n 4 \times 4}$ que representa $H_{\mathcal{N}}$ nesta nova base é

$$
H_{n 4 \times 4}=\left(\begin{array}{cccc}
\epsilon_{0} & 0 & 0 & 0 \\
0 & \epsilon_{1} & 0 & 0 \\
0 & 0 & \epsilon_{0} & 0 \\
0 & 0 & 0 & \epsilon_{1}
\end{array}\right) .
$$

Passarei a trabalhar com a representação matricial de cada termo da hamiltoniana para, no final dos cálculos, diagonalizar simultaneamente todos os termos de $H_{\text {eff }}$ (representada por $H_{\text {eff } 4 \times 4}$ ). Este procedimento toma como hipótese que a base de $H_{\mathcal{N}}$ é suficiente para representar $H_{\text {eff }}$, o que pode ser bem razoável frente ao fato de que todos os termos presentes em $H_{\text {eff }}$ que não estão em $H_{\mathcal{N}}$ são de segunda ordem em $1 / E_{g}$. 


\subsection{Acoplamentos intra- e intersub-bandas}

Com a diagonalização proposta na seção 3.2, todos os termos da hamiltoniana são diagonais em spin, incluindo $H_{s o}$ (definido na equação 2.7.37). A seguir, calcularei os elementos de matriz do acoplamento spin-órbita na base $\left\{\left|u_{0}+\right\rangle,\left|u_{1}+\right\rangle,\left|u_{0}-\right\rangle,\left|u_{1}-\right\rangle\right\}$.

Tomando os elementos de matriz de $H_{s o}$,

$$
\left\langle u_{j} \mu\left|H_{s o}\right| u_{p} \nu\right\rangle=\left\langle u_{j} \mu\left|\eta(z)\left\{k_{x} \sigma_{y}-k_{y} \sigma_{x}\right\}\right| u_{p} \nu\right\rangle
$$

Note que $H_{s o}$ é um caso do produto 3.1.10, em que dividirei os operadores da seguinte forma:

$$
H_{s o}=\eta(z) k_{x} \otimes \sigma_{y}-\eta(z) k_{y} \otimes \sigma_{x}
$$

Dessa forma,

$$
\begin{gathered}
\left\langle u_{j} \mu\left|H_{s o}\right| u_{p} \nu\right\rangle=\left\langle u_{j} \mu\left|\eta(z) k_{x} \otimes \sigma_{y}\right| u_{p} \nu\right\rangle-\left\langle u_{j} \mu\left|\eta(z) k_{y} \otimes \sigma_{x}\right| u_{p} \nu\right\rangle \\
\therefore\left\langle u_{j} \mu\left|H_{s o}\right| u_{p} \nu\right\rangle=\left\langle u_{j}|\eta(z)| u_{p}\right\rangle\left\langle\mu\left|k_{x} \sigma_{y}-k_{y} \sigma_{x}\right| \nu\right\rangle .
\end{gathered}
$$

Os acoplamentos spin-órbita são representados por uma matriz $\eta$, cujos elementos são

$$
\eta_{j p}=\left\langle u_{j}|\eta(z)| u_{p}\right\rangle
$$

Quando $j=p$ (elementos da diagonal), então o elemento de matriz refere-se à mesma autofunção de $H_{\mathcal{N}}$ e, portanto, entre mesmas sub-bandas. Por isso, este acoplamento é conhecido como acoplamento intrasub-bandas. No caso de apenas uma sub-banda, este é o único acoplamento spin-órbita que existe. Foi estudado pela primeira vez por Rashba, e por este nome é vastamente conhecido na literatura. Além desse nome, por este acoplamento ser gerado pela assimetria no potencial estrutural (induzido por campos externos), também costuma-se chamar $\eta_{j j}$ de acoplamento por assimetria de inversão estrutural - ou SIA (como comentamos, do inglês structral inversion asymetry).

Quando $j \neq p$ (elementos na diagonal secundária), $\eta_{j p}$ acopla duas diferentes sub-bandas, e por isso é conhecido como acoplamento intersub-bandas. Este foi o tema principal dos trabalhos $^{3,4}$ e gerou recente interesse principalmente por gerar novos efeitos interessantes (zitterbewegung não usual, ressonância em poços quânticos duplos ${ }^{35}$ e efeito Hall de spin não nulo $^{2,5}$ ) e por ainda não haver muitos experimentos com duas sub-bandas. Da hermitianicidade de $H_{s o}, \eta_{01}=\eta_{10}$. Na figura 3.3, expus algumas estimativas destes acoplamentos novamente para $\operatorname{InSb} / A l I n S b$. 


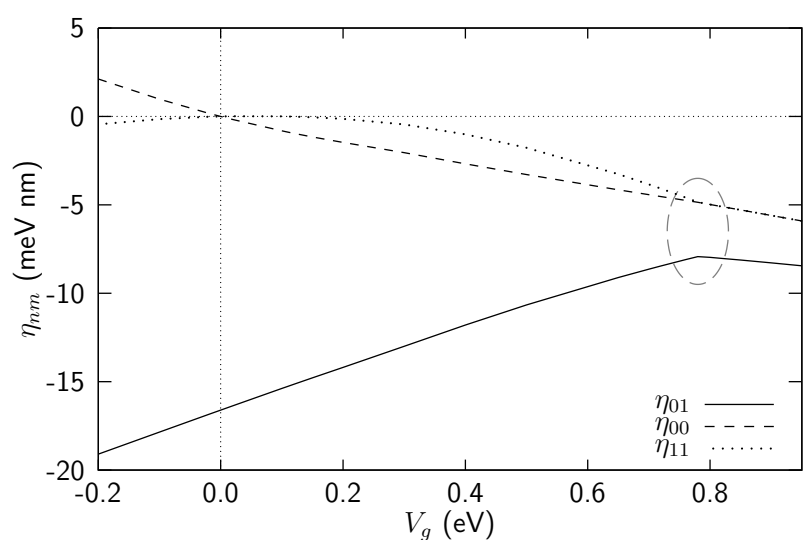

(a) $\eta_{n m}$ em função dos gates.

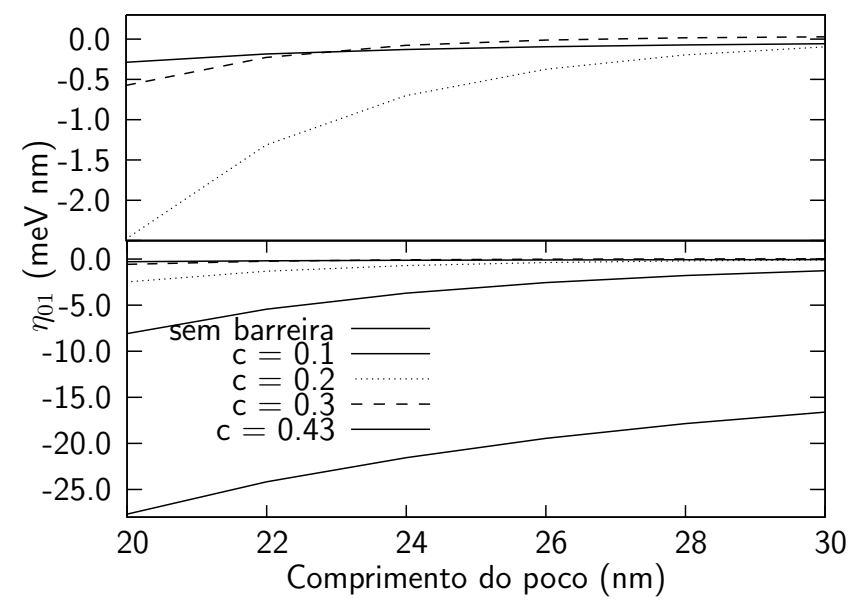

(b) Acoplamento intersub-banda com barreiras

Figura 3.3 - Relação entre o novo acoplamento spin-órbita e a largura do poço para diversas concentrações de impurezas na barreira central (inclusive, sem barreira). As diferentes concentrações, marcadas com $c$, foram $10 \%, 20 \%, 30 \%$ e $43 \%$. Como As paredes do poço quântico são formadas pela mesma substância da barreira, mas com concentração $38 \%$, a barreira está acima do nível do poço. Todos os outros parâmetros foram mantidos os mesmos, e o gate foi fixado nulo. Relação entre os acoplamentos spin-órbita e as tensões aplicadas nos gates para a estrutura $A l_{0.38} I n_{0.62} S b$, com potencial químico $70 \mathrm{eV}$ e poço de $30 \mathrm{~nm}$.

Podemos representar matricialmente os elementos da equação 3.3.3 como

$$
H_{s o 4 \times 4}=\left(\begin{array}{cccc}
\eta_{00} k_{\|} & \eta_{01} k_{\|} & 0 & 0 \\
\eta_{01} k_{\|} & \eta_{00} k_{\|} & 0 & 0 \\
0 & 0 & -\eta_{00} k_{\|} & -\eta_{01} k_{\|} \\
0 & 0 & -\eta_{01} k_{\|} & -\eta_{00} k_{\|}
\end{array}\right) .
$$

Fantástico, acabamos com uma hamiltoniana bloco-diagonalizada. O importante será verificar se os outros termos de $H_{\mathcal{A}}$ destruirão este formato extremamente confortável.

\subsection{Poço perfeitamente retangular}

Se o poço for perfeitamente retangular, i.e., com interfaces abruptas entre cada camada semicondutora, então fica bem mais simples derivar algumas expressões analíticas para o acoplamento momento-campo elétrico e o termo de Darwin. A equação 2.4.2 descreve muito bem o perfil de um poço abrupto usando funções de Heaviside. O perfil de potencial $h_{w}$ depende de funções de Heaviside, e os termos na hamiltoniana dependem de derivadas de perfil. Lembrando que

$$
\frac{d \mathfrak{H}}{d z}(z)=\delta(z),
$$


substituirei $h_{w}$ nas expressões do acoplamento momento-campo elétrico e do termo de Darwin e os representarei matricialmente.

\subsubsection{Acoplamento momento-campo elétrico}

Utilizarei a sigla MEC (de Momento-Campo Elétrico) para fazer referências a este acoplamento. O primeiro termo na equação 3.1 .5 pode ser reescrito como

$$
H_{M E C}:=-\alpha_{M} \frac{d h_{w}}{d z}(z) \frac{d}{d z}
$$

Usando a definição 2.4.2,

$$
\frac{d h_{w}}{d z}(z)=\frac{d}{d z}\left\{1+\mathfrak{H}\left(z-a_{0}\right)-\mathfrak{H}\left(z+a_{0}\right)\right\}=\delta\left(z-a_{0}\right)-\delta\left(z+a_{0}\right) .
$$

Com isso, obtemos

$$
H_{M E C}=-\alpha_{M}\left[\delta\left(z-a_{0}\right)-\delta\left(z+a_{0}\right)\right] \frac{d}{d z} .
$$

Utilizando a base proposta na seção 3.2, os elementos de matriz de 3.4 .4 ficam

$$
\begin{gathered}
\left\langle n \mu\left|H_{M E C}\right| m \nu\right\rangle=\left\langle n\left|H_{M E C}\right| m\right\rangle \delta_{\mu, \nu}=-\alpha_{M} \delta_{\mu, \nu} \int d z\langle n \mid z\rangle\left[\delta\left(z-a_{0}\right)-\delta\left(z+a_{0}\right)\right] \frac{d}{d z}\langle z \mid m\rangle \\
-\alpha_{M} \delta_{\mu, \nu} \int d z u_{n}(z)\left[\delta\left(z-a_{0}\right)-\delta\left(z+a_{0}\right)\right] \frac{d u_{m}}{d z}(z)= \\
H_{M E C}^{n m}:=\left\langle n \mu\left|H_{M E C}\right| m \nu\right\rangle=-\alpha_{M} \delta_{\mu, \nu}\left[u_{n}\left(a_{0}\right) \frac{d u_{m}}{d z}\left(a_{0}\right)-u_{n}\left(-a_{0}\right) \frac{d u_{m}}{d z}\left(-a_{0}\right)\right]
\end{gathered}
$$

Como $H_{M E C}^{n m}$ é real, então $H_{M E C}^{01}=H_{M E C}^{10}$. Note que estes elementos de matriz dependem fortemente da assimetria do poço (gates aplicados). Para estimar os valores de $H_{s}^{m n}$ numericamente, farei uso de meus programas (comentados na seção 3.1.2). Na figura 3.4 mostro a dependência deste elemento de matriz tanto com o gate como com a largura do poço. Nos gráficos que apresento na figura 3.4, o acoplamento momento-campo elétrico intersub-bandas é desprezível perto do acoplamento intersub-bandas, $H_{M E C}^{01} / \eta_{01} \approx 0.1$ nos melhores casos. Já os acoplamentos intrasub-bandas do momento-campo elétrico apresentam valores apreciáveis.

Como a aplicação de gates altera as funções $u_{j}$, os elementos $H_{s}^{n m}$ dependem do gate (comprovado na figura 3.4(a)). Se o gate é nulo (caso simétrico), então as soluções do poço quântico têm paridade definida ${ }^{79}: u_{0}$ é uma função par e $u_{1}$, ímpar. Disso segue que $d u_{0} / d z$ é uma função ímpar e $d u_{1} / d z$, par. Assim,

$$
u_{0}\left(-a_{0}\right)=u_{0}\left(a_{0}\right) \quad \text { e } \quad u_{1}\left(-a_{0}\right)=-u_{1}\left(a_{0}\right)
$$




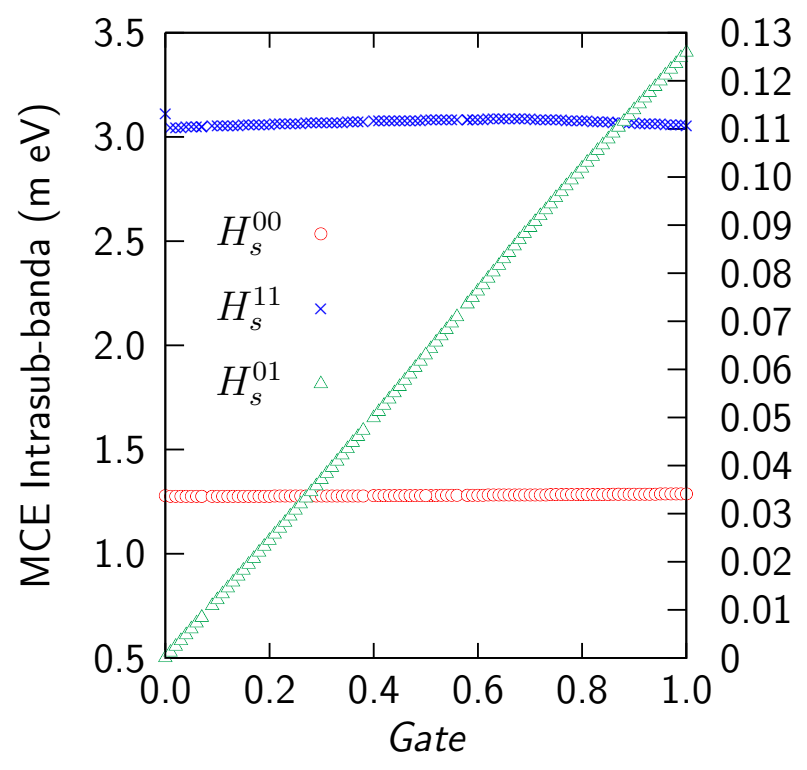

(a) Dependência com os gates

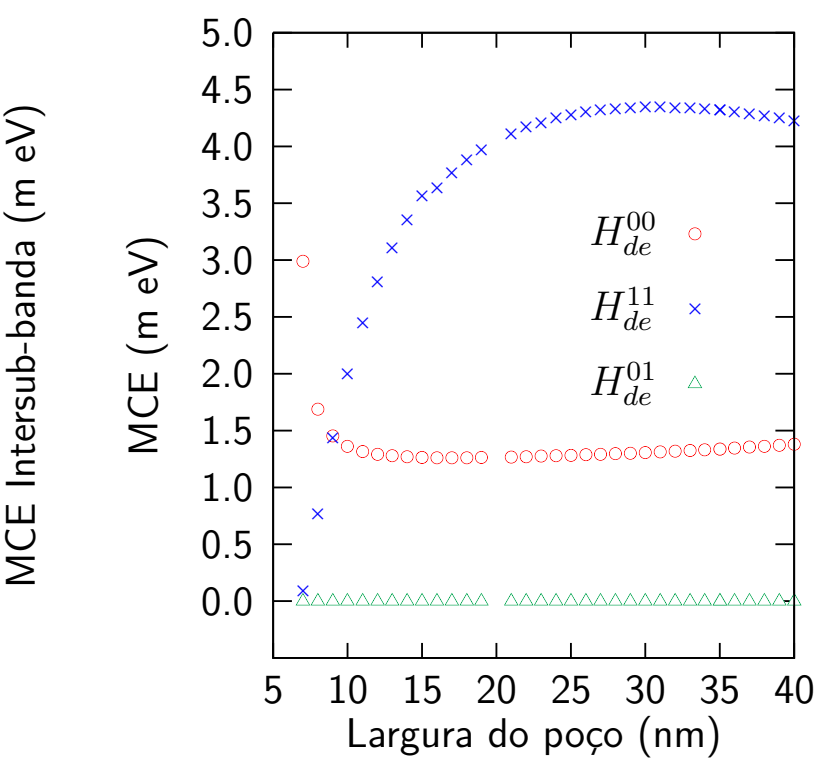

(b) Largura do poço (caso simétrico)

Figura 3.4 - Dependência do elemento de matriz do termo do acoplamento Momento-Campo Elétrico (MCR nos gráficos) tanto com o gate aplicado como com a largura do poço quântico. Podemos notar que os acoplamentos intrasub-bandas praticamente não variam com os gates, enquanto que o acoplamento intersub-bandas varia linearmente. Já com a largura do poço, vemos que os acoplamentos intersub-bandas é altamente controlável. Podemos também ver o desaparecimento do acoplamento intersub-bandas no caso simétrico.

e, consequentemente,

$$
\frac{d u_{0}}{d z}\left(-a_{0}\right)=-\frac{d u_{0}}{d z}\left(a_{0}\right) \quad \text { e } \quad \frac{d u_{1}}{d z}\left(-a_{0}\right)=\frac{d u_{1}}{d z}\left(a_{0}\right) .
$$

Portanto, no caso simétrico vale

$$
\begin{aligned}
& H_{M E C}^{00} \propto u_{0}\left(a_{0}\right) \frac{d u_{0}}{d z}\left(a_{0}\right)-u_{0}\left(a_{0}\right)\left(-\frac{d u_{0}}{d z}\left(a_{0}\right)\right)=2 u_{0}\left(a_{0}\right) \frac{d u_{0}}{d z}\left(a_{0}\right), \\
& H_{M E C}^{11} \propto u_{1}\left(a_{0}\right) \frac{d u_{1}}{d z}\left(a_{0}\right)-\left(-u_{1}\left(a_{0}\right)\right) \frac{d u_{1}}{d z}\left(a_{0}\right)=2 u_{1}\left(a_{0}\right) \frac{d u_{1}}{d z}\left(a_{0}\right) \\
& H_{M E C}^{01} \propto u_{0}\left(a_{0}\right) \frac{d u_{1}}{d z}\left(a_{0}\right)-\left(-u_{0}\left(a_{0}\right)\right)\left(-\frac{d u_{1}}{d z}\left(a_{1}\right)\right)=0=H_{M E C}^{10}
\end{aligned}
$$

Os elementos $H_{M E C}^{01}$ e $H_{M E C}^{01}$ são nulos para poços simétricos, caso em que emergiu o estudo do acoplamento intersub-bandas ${ }^{3}$. Estes dois elementos de matriz são calculados entre duas sub-bandas diferentes, por isso é um tipo de acoplamento intersub-banda. Os dois elementos da diagonal, que podem ser chamados de intrasub-bandas, não se anulam mesmo quando não há gates aplicados. Este comportamento é similar, embora invertido, ao acoplamento spinórbita inter e intrasubbandas.

Podemos representar esta nova contribuição matricialmente, na base $\{|0+\rangle,|1+\rangle,|0-\rangle,|1-\rangle\}$ 
como

$$
H_{s 4 \times 4}=\left(\begin{array}{cccc}
H_{M E C}^{00} & H_{M E C}^{01} & 0 & 0 \\
H_{M E C}^{01} & H_{M E C}^{11} & 0 & 0 \\
0 & 0 & H_{M E C}^{00} & H_{M E C}^{01} \\
0 & 0 & H_{M E C}^{01} & H_{M E C}^{11}
\end{array}\right) .
$$

Novamente, a matriz já está na forma bloco diagonal. Se reunirmos esta representação matricial e $H_{s o 4 \times 4}$, da equação 3.3.5, podemos interpretar a ação de $H_{M E C}^{10}$ de forma interessante,

$$
\begin{aligned}
& H_{M E C 4 \times 4}+H_{s o 4 \times 4}= \\
& \left.\qquad \begin{array}{cccc}
\epsilon_{0}-\eta_{00} k_{\|}+H_{s}^{00} & -\eta_{01}\left(k_{\|}-\frac{H_{s}^{01}}{\eta_{01}}\right) & 0 & 0 \\
-\eta_{01}\left(k_{\|}-\frac{H_{s}^{01}}{\eta_{01}}\right) & \epsilon_{1}-\eta_{00} k_{\|}+H_{s}^{11} & 0 & 0 \\
0 & 0 & \epsilon_{0}+\eta_{00} k_{\|}+H_{s}^{00} & -\eta_{01}\left(k_{\|}-\frac{H_{M E C}^{01}}{\eta_{01}}\right) \\
0 & 0 & -\eta_{01}\left(k_{\|}-\frac{H_{M E C}^{01}}{\eta_{01}}\right) & \epsilon_{1}+\eta_{00} k_{\|}+H_{M E C}^{11}
\end{array}\right) .
\end{aligned}
$$

A unidade de $H_{M E C}^{01} / \eta$ é igual a $m^{-1}$, ou seja, os termos não diagonais são como deslocamentos entre os vetors de onda $k_{\| 1}$ que acompanham o acoplamento intersub-banda dos que acompanham o intrasub-banda. Naturalmente, no caso simétrico este deslocamento é nulo. Além disso, como observado na figura 3.4, $H_{M E C}^{01} / \eta$ é muito pequeno e portanto não deve gerar grandes diferenças. Por fim, reunindo estas duas contribuições com a diagonal 3.2.6 (isto é, deixando apenas Darwin de fora), obtemos

$$
\begin{aligned}
& H_{n 4 \times 4}+H_{s o 4 \times 4}+H_{M E C 4 \times 4}= \\
& \left(\begin{array}{cccc}
\tilde{\epsilon}_{0}+\eta_{00} k_{\| 1} & \eta_{01}\left(k_{\|}-\frac{H_{M E C}^{01}}{\eta}\right) & 0 & 0 \\
\eta_{01}\left(k_{\|}-\frac{H_{M E C}^{01}}{\eta}\right) & \tilde{\epsilon}_{1}+\eta_{00} k_{\|} & 0 & 0 \\
0 & 0 & \tilde{\epsilon}_{0}-\eta_{00} k_{\| 1} & -\eta_{01}\left(k_{\|}-\frac{H_{M E C}^{01}}{\eta}\right) \\
0 & 0 & -\eta_{01}\left(k_{\|}-\frac{H_{M E C}^{01}}{\eta}\right) & \tilde{\epsilon}_{1}-\eta_{00} k_{\|}
\end{array}\right),
\end{aligned}
$$

em que defini implicitamente

$$
\tilde{\epsilon}_{j}=\epsilon_{j}+H_{M E C}^{j j} .
$$

Como o que interessa de fato são diferenças de energia,

$$
\Delta E:=\tilde{\epsilon}_{1}-\tilde{\epsilon}_{0}=\epsilon_{1}-\epsilon_{2}+H_{M E C}^{11}-H_{M E C}^{00}
$$

Portanto, os termos diagonais podem ser compreendidos como deslocamentos adicionais nas 
energias das soluções de $H_{\mathcal{N}}$. Estes, em contraponto com $H_{M E C}^{01} / \eta$, não são desprezíveis e podem gerar efeitos mais visíveis

\subsubsection{Correção estrutural análoga à de Darwin}

O termo de Darwin leva em conta a contribuição de dois potenciais completamente distintos: o estrutural $h_{w}$ e a interação entre elétrons $V$. Investigarei separadamente cada uma destas duas contribuições. Nesta seção, dedicarei nossa atenção à contribuição devido ao potencial estrutural, que pode ser reescrito na base $|z\rangle$ como

$$
H_{d e}:=\alpha_{D} \delta_{6} \frac{d^{2} h_{w}}{d z^{2}}(z)
$$

com $\alpha_{D}$ definido em 3.1.2a. Vale lembrar ao leitor que as constantes $\alpha_{D}$ e $\alpha_{M E C}$ carregam sinais negativos explícitos. Calculando explicitamente, obtemos

$$
\begin{gathered}
H_{d e}=\alpha_{D} \delta_{6} \frac{d}{d z}\left[\frac{d \mathfrak{H}}{d z}\left(z-a_{0}\right)-\frac{d \mathfrak{H}}{d z}\left(z+a_{0}\right)\right]=\alpha_{D} \delta_{6}\left[\frac{d \delta}{d z}\left(z-a_{0}\right)-\frac{d \delta}{d z}\left(z+a_{0}\right)\right] \\
=\delta_{6} \alpha_{D}\left\{\delta^{\prime}\left(z-a_{0}\right)-\delta^{\prime}\left(z+a_{0}\right)\right\}
\end{gathered}
$$

em que $\delta^{\prime}(z)$ é a primeira derivada da distribuição de Dirac. Esta distribuição $\delta^{\prime}$ segue regras baseadas nas da distribuição de Dirac. Para uma função teste $f(z)$ com suporte finito, é possível mostrar que ${ }^{\S}$

$$
\int \delta^{\prime}(z-x) f(z) d z=-f^{\prime}(x) .
$$

Com isso, da mesma forma como calculamos os elementos de matriz de $H_{M E C}$, calcularei os elementos de matriz de $H_{d e}$, que serão divididos em intra e intersub-bandas. Como $H_{D}$ em si é diagonal para spin, podemos usar desde já a base $\{|0+\rangle,|1+\rangle,|0-\rangle,|1-\rangle\}$. Assim,

$$
\begin{aligned}
& H_{d e}^{n m}:=\left\langle n \mu\left|H_{d e}\right| m \nu\right\rangle= \\
& \alpha_{D} \delta_{6}\left[\int d z \phi_{n}(z) \phi_{m}(z) \delta^{\prime}\left(z-a_{0}\right)-\int d z \phi_{n}(z) \phi_{m}(z) \delta^{\prime}\left(z+a_{0}\right)\right] .
\end{aligned}
$$

\footnotetext{
§Propriedades da Delta de Dirac só podem ser derivadas com conhecimentos mais específicos de distribuições. Esta propriedade no entanto pode ser compreendida por uma integração por partes. Sugiro a leitura das discussões de Boykin ${ }^{80}$ para uma implementação autoconsistente deste termo.
} 
Definindo $g(z):=\phi_{n}(z) \phi_{m}(z)$, e então

$$
\begin{gathered}
H_{d e}^{n m}=\alpha_{D} \delta_{6}\left[\int d z g(z) \delta^{\prime}\left(z-a_{0}\right)-\int d z g(z) \delta^{\prime}\left(z+a_{0}\right)\right]=-\alpha_{D} \delta_{6}\left[\frac{d g}{d z}\left(a_{0}\right)-\frac{d g}{d z}\left(-a_{0}\right)\right]= \\
-\delta_{6}\left[\phi_{n}\left(a_{0}\right) \frac{d \phi_{m}}{d z}\left(a_{0}\right)+\phi_{m}\left(a_{0}\right) \frac{d \phi_{n}}{d z}\left(a_{0}\right)-\right. \\
\left.\phi_{n}\left(-a_{0}\right) \frac{d \phi_{m}}{d z}\left(-a_{0}\right)-\phi_{m}\left(-a_{0}\right) \frac{d \phi_{n}}{d z}\left(-a_{0}\right)\right]
\end{gathered}
$$

Exponho estimativas para estes elementos de matriz, de forma similar aos cálculos para o acoplamento momento-campo elétrico, na figura 3.4.2. Novamente, o acoplamento intersubbandas parece desprezível se comparado ao intersub-bandas de spin-órbita, $H_{d e}^{01} / \eta_{01} \approx 0.3$ no melhor dos casos. No entanto, os acoplamentos intrasub-bandas são ainda maiores.

A figura 3.5(a) mostra uma certa dependência desta correção de Darwin com os gates aplicados, similar ao que observamos para o acoplamento momento-campo elétrico. No caso simétrico,

$$
\begin{aligned}
H_{d e}^{00} & \propto 4 \phi_{0}\left(a_{0}\right) \frac{d \phi_{0}}{d z}\left(a_{0}\right), \\
H_{d e}^{11} & \propto 4 \phi_{1}\left(a_{0}\right) \frac{d \phi_{1}}{d z}\left(a_{0}\right), \\
H_{d e}^{01} & =0=H_{d e}^{10} .
\end{aligned}
$$

Exatamente o mesmo que observamos para o momento-campo elétrico. Além disso, os gráficos da figura 5(b) são bem parecidos com 4(b).

Em termos matriciais, não há grandes novidades: $H_{d e}$ soma-se à $H_{S}$. Assim, podemos escrever finalmente

$$
H_{4 \times 4}=\left(\begin{array}{cccc}
\tilde{\epsilon}_{0}+\eta_{00} k_{\|} & \eta_{01}\left(k_{\|}+k_{0}\right) & 0 & 0 \\
\eta_{01}\left(k_{\|}+k_{0}\right) & \tilde{\epsilon}_{1}+\eta_{00} k_{\|} & 0 & 0 \\
0 & 0 & \tilde{\epsilon}_{0}-\eta_{00} k_{\|} & -\eta_{01}\left(k_{\|}+k_{0}\right) \\
0 & 0 & -\eta_{01}\left(k_{\|}+k_{0}\right) & \tilde{\epsilon}_{1}-\eta_{00} k_{\|}
\end{array}\right),
$$

em que definimos

$$
k_{0}=\frac{H_{d e}^{01}+H_{M E C}^{01}}{\eta}
$$

e

$$
\widetilde{\epsilon_{j}}=\epsilon_{j}+H_{d e}^{j j}+H_{M E C}^{j j} \cdot
$$

Novamente a matriz pode ser facilmente diagonalizada.

Por fim, note que $k_{0}=0$ no caso simétrico, já que tanto o termo de Darwin, como o 


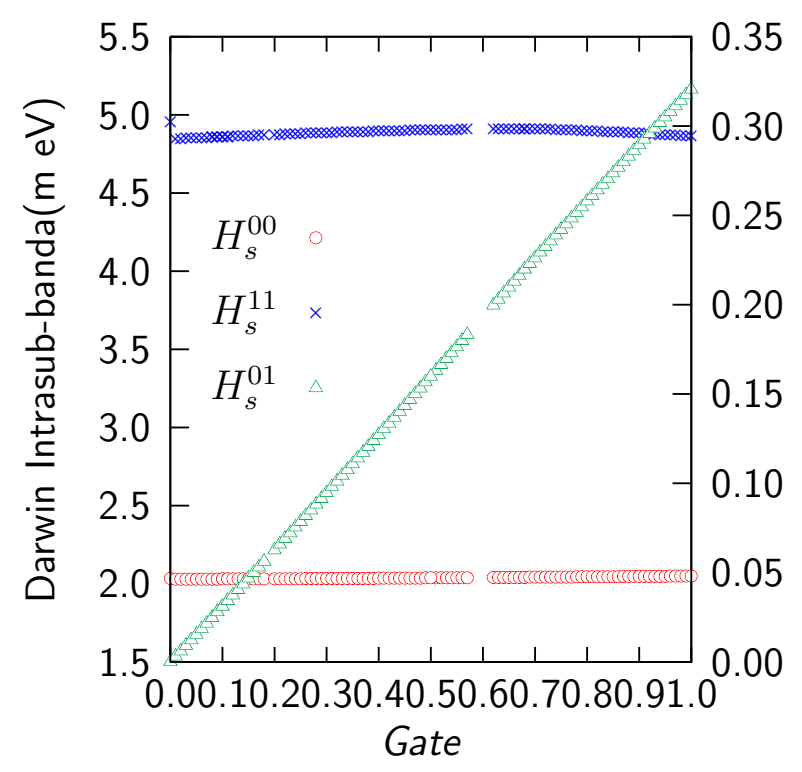

(a) Dependência com os gates

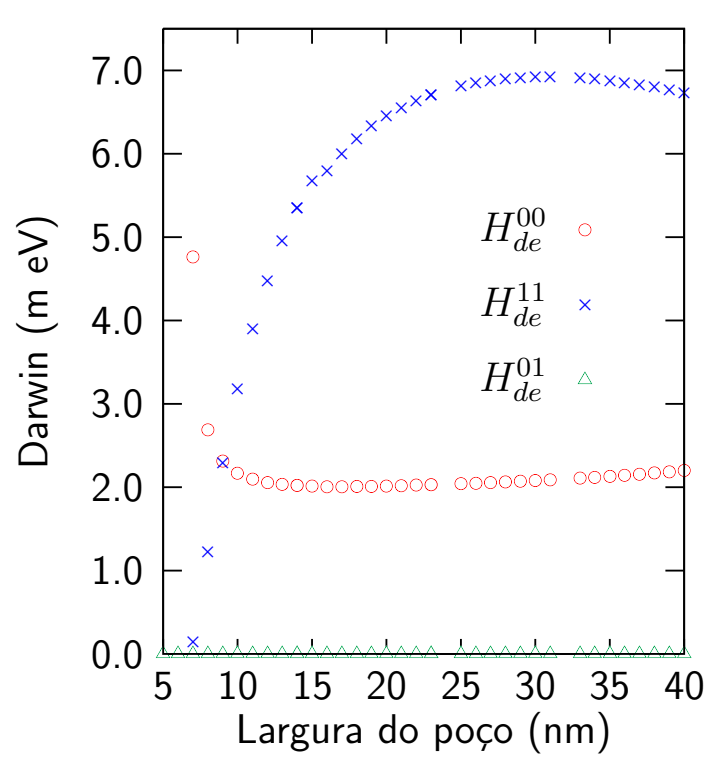

(b) Largura do poço (caso simétrico)

Figura 3.5 - Dependência do termo de Darwin tanto com o gate aplicado como com a largura do poço quântico. Fica evidente que as dependências são idênticas às que observamos para o acoplamento momento-campo elétrico, na figura 3.4. O elemento de matriz intersubbandas varia linearmente com os gates, e os intrasub-bandas não variam. Já com a largura do poço os elementos intrasub-bandas variam de forma considerável.

acoplamento momento-campo elétrico intersub-bandas são nulos. O mais significativo em deixar de lado estas contribuições independentes de spin está na abertura dos níveis em $\boldsymbol{k}_{\|}=$ $\mathbf{0}$, que depende dos elementos intrasub-bandas do momento-campo elétrico e do termo de Darwin.

\subsubsection{Correção do potencial eletrônico análoga à de Darwin}

Da equação de Poisson,

$$
\frac{d^{2} V}{d z^{2}}(z)=\frac{4 \pi e^{2}}{\kappa}\left[\rho_{e}(z)+\rho_{d}(z)\right],
$$

em que $\rho_{e}(z)$ é a distribuição eletrônica e $\rho_{d}$ é a distribuição de doadores ionizados. Por outro lado, podemos escrever

$$
\rho_{e}(z)=-2 e \sum_{j \boldsymbol{k}_{\|}} \frac{\left|u_{j}(z)\right|^{2}}{1+e^{\beta\left(\epsilon_{j}+\hbar^{2} k_{\|}^{2} / 2 m-\mu\right)}},
$$


em que $\mu$ é o potencial químico. O elemento de matriz deste termo fica

$$
H_{d V}^{n m}:=\left\langle n \mu\left|\frac{d^{2} V}{d z^{2}}\right| m \nu\right\rangle=\frac{4 \pi e^{2}}{\kappa}\left\langle n \mu\left|\rho_{e}\right| m \nu\right\rangle=\frac{4 \pi e^{2}}{\kappa} \delta_{\mu, \nu}\left\langle n\left|\rho_{e}\right| m\right\rangle,
$$

já usando $\left\langle n \mu\left|\rho_{d}\right| m \nu\right\rangle=0$ por $\rho_{d}(z)$ ser praticamente nulo nas regiões onde as funções de onda não são. Minhas rotinas numéricas já fornecem este elemento de matriz e deixamos como outro parâmetro advindo da simulação. Note que $H_{d V}^{n m}$ contribui de forma bem parecida a $H_{d e}^{n m}$. Para inclui-lo, é suficiente trocar $H_{d e}^{n m}$ por $H_{d e}^{n m}+H_{d V}^{n m}$.

No caso de poços simétricos, $u_{0}$ e $u_{1}$ têm paridades definidas. Portanto, como o valor absoluto de uma função ímpar é par, $\left\langle n\left|\rho_{e}\right| m\right\rangle=\delta_{m, n}\left\langle n\left|\rho_{e}\right| n\right\rangle$. Assim, $H_{d V}^{n m}$ segue o mesmo tipo de simplificação que $H_{d e}^{n m}$ no caso simétrico.

\subsection{Relação de dispersão}

Como montamos ao longo deste capítulo uma representação matricial para $H_{\text {eff }}$, podemos calcular seus autovalores para obter as relações de dispersões de cada um dos autoestados do poço. A seguir mostrarei estas soluções em separado para o caso de poços simétricos e assimétricos.

\subsubsection{Caso simétrico}

Por caso simétrico entenda uma estrutura sem a aplicação de gates externos. Isto anula diversas contribuições que calculei nas seções anteriores, e a Hamiltoniana final fica com a forma

$$
H_{4 \times 4}=\left(\begin{array}{cccc}
\tilde{\epsilon}_{0} & \eta_{01} k_{\|} & 0 & 0 \\
\eta_{01} k_{\|} & \tilde{\epsilon}_{1} & 0 & 0 \\
0 & 0 & \tilde{\epsilon}_{0} & -\eta_{01} k_{\|} \\
0 & 0 & -\eta_{01} k_{\|} & \tilde{\epsilon}_{1}
\end{array}\right) \text {, }
$$

com

$$
\widetilde{\epsilon_{j}}=\epsilon_{j}+H_{d e}^{j j}+H_{s}^{j j}
$$

Vale lembrar que eu já substitui $k_{0}=0$ por se tratar do caso simétrico. Há naturalmente quatro autovalores, dos quais dois referem-se exclusivamente ao primeiro bloco $2 \times 2$ e os outros dois aos segundo bloco. Isso separa $H_{4 \times 4}$ em dois subespaços distinos. Estes subespaços serão identificados por $\lambda_{1} \in\{1,-1\}$ - da ordenação da base, a diferença entre os blocos está no spin e, por isso, $\lambda_{1}$ pode ser encarado como número quântico de spin. Os autovalores $E\left(\boldsymbol{k}_{\| 1}, \lambda_{1}, \lambda_{2}\right)$ 
de $H_{4 \times 4}$ são dois a dois degenerados (um para cada subespaço),

$$
E\left(\boldsymbol{k}_{\|}, \lambda_{1}, \lambda_{2}\right)=\widetilde{\epsilon}_{+}+\lambda_{2} \Omega(k)
$$

em que defini

$$
\begin{aligned}
\widetilde{\epsilon}_{ \pm} & :=\frac{\widetilde{\epsilon_{1}} \pm \widetilde{\epsilon_{0}}}{2} \\
4 \Omega^{2}(k) & :=4 \widetilde{\epsilon}_{-}^{2}+4 \eta_{01}^{2}\left(k_{\text {॥ }}-k_{0}\right)^{2}
\end{aligned}
$$

A forma da relação de dispersão não é modificada, se comparada à de trabalhos anteriores ${ }^{3}$. Como $\lambda_{1}$ seleciona o subespaço de spin, então os estados são degenerados em spin. Esta degenerescência em spin só é quebrada com a introdução do acoplamento intrasub-bandas, i.e., quebra de simetria de inversão no potencial estrutural.

\subsubsection{Caso geral}

Quando há assimetria de inversão no potencial estrutural, algumas contribuições nulas do caso simétrico vêm a tona. Por isso, podemos entender o caso assimétrico como um caso mais geral: anulando-se estes termos extras, reobtemos o caso simétrico. A hamiltoniana $H_{4 \times 4}$ pode ser escrita como

$$
H_{4 \times 4}=\left(\begin{array}{cccc}
\tilde{\epsilon}_{0}+\eta_{00} k_{\|} & \eta_{01}\left(k_{\|}-k_{0}\right) & 0 & 0 \\
\eta_{01}\left(k_{\|}-k_{0}\right) & \tilde{\epsilon}_{1}+\eta_{11} k_{\|} & 0 & 0 \\
0 & 0 & \tilde{\epsilon}_{0}-\eta_{00} k_{\|} & -\eta_{01}\left(k_{\|}-k_{0}\right) \\
0 & 0 & -\eta_{01}\left(k_{\|}-k_{0}\right) & \tilde{\epsilon}_{1}-\eta_{11} k_{\|}
\end{array}\right),
$$

e seus autovalores são

$$
E\left(\boldsymbol{k}_{\|}, \lambda_{1}, \lambda_{2}\right)=\widetilde{\epsilon}_{+}-\lambda_{1} \alpha_{+} k_{\|}+\lambda_{2} \Omega_{\lambda_{1}}\left(k_{\| 1}\right),
$$

em que defini implicitamente

$$
\begin{aligned}
\alpha_{ \pm} & :=\frac{\eta_{11}-\eta_{00}}{2} \\
\Omega_{\lambda_{1}}^{2}(k) & :=\left(2 \widetilde{\epsilon}_{-}+\lambda_{1} \alpha_{-} k_{\|}\right)^{2}+\eta_{01}^{2}\left(k_{\text {॥ }}-k_{0}\right)^{2} .
\end{aligned}
$$

Note que a energia depende de $\lambda_{1}$, ou seja, a assimetria quebra a degenerescência por spin. A figura 3.6 mostra pictoricamente este desdobramento. 


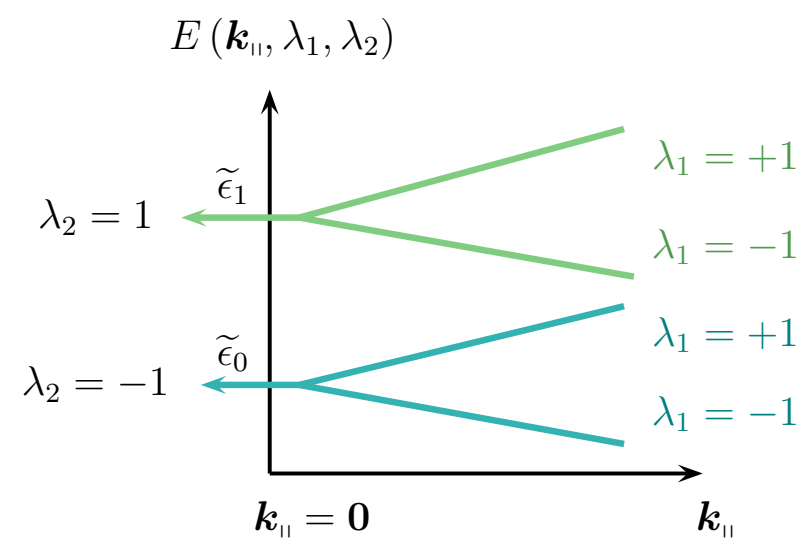

Figura 3.6 - Desdobramento pictórico dos níveis de energia próximo ao ponto $\Gamma$. O número quântico $\lambda_{2}$ seleciona a sub-banda, que se afastam por energias da ordem de centésimos ou décimos de elétron-volts. O número quântico $\lambda_{1}$ abre os níveis, com uma diferença de energias de alguns décimos de milésimos de elétron-volts, e que definimos na equação 3.5.8. No caso simétrico, os níveis separados por $\lambda_{1}$ são degenerados.

O famoso splitting de Rashba é observado na quebra de degenerescência de spin:

$$
\Delta_{\lambda_{2}}\left(\boldsymbol{k}_{\|}\right)=E\left(\boldsymbol{k}_{\|}, 1, \lambda_{2}\right)-E\left(\boldsymbol{k}_{\|},-1, \lambda_{2}\right)
$$

é a diferença de energia para a sub-banda $\lambda_{2}$. Para diversos materiais, estas aberturas são da ordem de décimos de milésimos de eletron-volts ${ }^{73}$. Esta é a diferença entre os níveis desdobrados na figura 3.6.

\subsection{A banda de condução}

A seguir mostrarei os autoestados associados às relações de dispersão calculadas nesta última seção. Vale lembrar que a banda de condução é o spinor $\left(\psi_{1}, \psi_{2}\right)^{T}$ na base $\{|\uparrow\rangle,|\downarrow\rangle\}$. Todos estes resultados foram apresentados em dois congressos internacionais ${ }^{81,82}$.

\subsubsection{Caso simétrico}

A equação efetiva para a banda de condução, no caso simétrico, pode ser escrita matricialmente como

$$
H_{4 \times 4}\left|\lambda_{1} \lambda_{2}\right\rangle=E\left(\boldsymbol{k}_{\| 1}, \lambda_{1}, \lambda_{2}\right)\left|\lambda_{1} \lambda_{2}\right\rangle
$$

em que $H_{4 \times 4}$ é dada pela equação 3.5.1 e $\left|\lambda_{1} \lambda_{2}\right\rangle$ é um vetor desconhecido de quatro componentes. Portanto, a equação 3.6.1 é um sistema de quatro equações lineares. Para calcular os 
autovetores, resolvi este sistema linear e obtive

$$
\left|\lambda_{1} \lambda_{2}\right\rangle=\left(\frac{\eta_{01} k_{\|}}{\Omega(k)+\lambda_{2} \widetilde{\epsilon}_{-}}\left|u_{0}\right\rangle+\left|u_{1}\right\rangle\right) \otimes\left|\lambda_{1}\right\rangle .
$$

Apesar de $u_{0}$ e $u_{1}$ serem funções com paridade definida, as soluções $\left\langle z \mid \lambda_{1} \lambda_{2}\right\rangle$ não têm paridade definida. É possível mostrar, de forma bem simples, que

$$
\left\langle\lambda_{1}^{\prime} \lambda_{2}^{\prime} \mid \lambda_{1} \lambda_{2}\right\rangle=\delta_{\lambda_{1}, \lambda_{1}^{\prime}} \delta_{\lambda_{2}, \lambda_{2}^{\prime}}
$$

como se esperaria de soluções de um operador hermitiano. Para termos um resultado mais completo, incluirei na hamiltoniana os acoplamentos spin-órbita intrasub-bandas e os intersubbandas do termo de Darwin e o acoplamento momento-campo elétrico.

\subsubsection{Caso geral}

No caso geral, em que os acoplamentos intersub-bandas $\eta_{j j}$ não são nulos, a hamiltoniana é dada por 3.5.5. Analogamente, obtive como autovetores

$$
\left|\lambda_{1} \lambda_{2}\right\rangle=\left(\frac{\eta_{01}\left(k_{\| 1}-k_{0}\right)}{\lambda_{2} \Omega_{\lambda_{1}}\left(k_{\|}\right)+\left(\widetilde{\epsilon}_{-}+\lambda_{1} \alpha_{-}\right)}\left|u_{0}\right\rangle+\left|u_{1}\right\rangle\right) \otimes\left|\lambda_{1}\right\rangle .
$$

Não é muito difícil mostrar que, para $\eta_{00}=\eta_{11}=0$ e $H_{d e}^{00}=H_{d e}^{11}=0$, a solução apresentada na equação 3.6.5 iguala-se à da equação 3.6.2, ou seja, o caso simétrico é um caso particular do caso assimétrico.

Originalmente, nós buscávamos as funções envelope da banda de condução. Os autovetores 3.6.4 não são estas funções, mas estão certamente ligados às suas projeções em $\langle\boldsymbol{r}|$. Calculando a projeção dos autovetores 3.6.4 na base do operador posição, obtemos

$$
\left\langle z \mid \lambda_{1} \lambda_{2}\right\rangle=\left(\frac{\eta_{01}\left(k-k_{0}\right)}{\lambda_{2} \Omega_{\lambda_{1}}(k)+\left(\widetilde{\epsilon}_{-}+\lambda_{1} \alpha_{-}\right)} u_{0}(z)+u_{1}(z)\right) \frac{\lambda_{1} e^{-i \theta}|\uparrow\rangle+|\downarrow\rangle}{\sqrt{2}} .
$$

As funções envelope $\psi_{1}$ e $\psi_{2}$ ganham os índices $\lambda_{1}$ e $\lambda_{2}$. Como a parte dependente em $z$ está isolada, defini a função

$$
f_{\lambda_{1} \lambda_{2}}(z):=\frac{\eta_{01}\left(k_{\| 1}-k_{0}\right)}{\lambda_{2} \Omega_{\lambda_{1}}\left(k_{\text {॥l }}\right)+\left(\widetilde{\epsilon}_{-}+\lambda_{1} \alpha_{-}\right)} u_{0}(z)+u_{1}(z),
$$

『Subentenda $| \pm 1\rangle=| \pm\rangle$. 
e assim

$$
\begin{aligned}
& \psi_{1 \lambda_{1} \lambda_{2}}(z)=\frac{\lambda_{1} e^{-i \theta}}{\sqrt{2}} f_{\lambda_{1} \lambda_{2}}(z) \\
& \psi_{2 \lambda_{1} \lambda_{2}}(z)=\frac{1}{\sqrt{2}} f_{\lambda_{1} \lambda_{2}}(z)
\end{aligned}
$$

Para mais detalhes sobre esta identificação, sugiro a leitura do apêndice D.

Existe uma escolha claramente arbitrária em 3.6.7, criada com os autovetores da equação D.1 do acoplamento spin-órbita. Devido a uma particular escolha, apenas $\phi_{1 \lambda_{1} \lambda_{2}}$ é complexo,

$$
\phi_{1 \lambda_{1} \lambda_{2}}(z)=\frac{\lambda_{1}}{k \sqrt{2}}\left(k_{x}-i k_{y}\right) f_{\lambda_{1} \lambda_{2}}(z)
$$

e $\phi_{2 \lambda_{1} \lambda_{2}}$ é puramente real.

Por fim, vou reescrever os autoestados da banda de condução de uma forma mais compacta,

$$
\left|\lambda_{1} \lambda_{2}\right\rangle=A_{\lambda_{1} \lambda_{2}}^{\boldsymbol{k}_{\|}}\left(A_{\lambda_{1} \lambda_{2}}^{\boldsymbol{k}_{\|}}\left|u_{0}\right\rangle+\left|u_{1}\right\rangle\right) \otimes\left|\lambda_{1}\right\rangle
$$

em que defino implicitamente

$$
B_{\lambda_{1} \lambda_{2}}^{\boldsymbol{k}_{\|}}=\frac{\eta_{01}\left(k-k_{0}\right)}{\lambda_{2} \Omega_{\lambda_{1}}(k)+\left(\widetilde{\epsilon}_{-}+\lambda_{1} \alpha_{-}\right)} .
$$

e a normalização $A_{\lambda_{1} \lambda_{2}}^{\boldsymbol{k}_{\|}}=\left\{1+\left(B_{\lambda_{1} \lambda_{2}}^{\boldsymbol{k}_{\|}}\right)^{2}\right\}^{-1 / 2}$. Esta forma será muito utilizada no capítulo 5 por causa do tamanho de algumas expressões.

\subsection{Caso com uma sub-banda}

Finalizando esta discussão, vou me restringir rapidamente à existência de apenas uma sub-banda. Este é o caso famoso em que emerge apenas o acoplamento de Rashba. Suponha que o poço quântico é construído de forma que caiba apenas um único estado ligado - ou seja, uma única sub-banda pode ser alocada neste poço. Utilizarei exatamente a mesma notação deste capítulo.

Neste caso, usamos apenas uma das duas soluções autoconsistentes: $u_{0}$. Neste caso, construí a base $\left\{\left|u_{0}+\right\rangle,\left|u_{0}-\right\rangle\right\} \|$. A hamiltoniana efetiva torna-se

$$
H_{\mathrm{eff}}=\left[\frac{\hbar^{2} k^{2}}{2 m}+h_{6}+V+\alpha_{D} k^{2}\left(\delta_{6} h_{w}+V\right)+\alpha_{s}\left(k_{z} h_{w}\right) \cdot k_{z}\right] \mathbb{1}_{2 x 2}-\alpha k_{\|} \sigma_{z},
$$

\footnotetext{
"Não há porquê utilizarmos a banda antiga de spin, se já podemos diagonalizar o acoplamento spin-órbita
} diretamente. 
em que $\alpha=\left\langle u_{0}|\eta| u_{0}\right\rangle=\eta_{00}$ é a constante de acoplamento de Rashba**. Todos os outros termos podem ser tratados exatamente como feito na seção 3.4, restringindo os cálculos a apenas uma sub-banda. As energias ficam

$$
E\left(\boldsymbol{k}_{\|}, \lambda_{1}\right)=\widetilde{\epsilon}-\lambda_{1} \alpha k_{\|}
$$

em que reutilizei a definição

$$
\widetilde{\epsilon}=\epsilon_{0}+H_{d e}^{00}+H_{s}^{00}
$$

Os autoestados podem ser calculados simplesmente resolvendo o sistema linear $H_{\text {eff }}\left|\lambda_{1}\right\rangle=$ $E\left(\boldsymbol{k}_{\|}, \lambda_{1}\right)\left|\lambda_{1}\right\rangle$. O resultado é bem trivial:

$$
\left|\lambda_{1}\right\rangle=\left|u_{0}\right\rangle \otimes\left|\lambda_{1}\right\rangle
$$

Note que isto é uma consequência direta da forma já diagonal apresentada pela hamiltoniana da equação 3.7.1. Se $\alpha_{D}=\alpha_{s}=0$, caímos no caso usualmente tratado na literatura.

\subsection{Hamiltoniana $4 \times 4$ e os campos magnéticos internos}

Para finalizar este capítulo, vamos rapidamente discutir um assunto que chamou muita atenção nos últimos anos, principalmente por constituir uma forma de medir os acoplamentos spin-órbita: os campos magnéticos internos. De volta por um momento à base de $\sigma_{z}$, a

\footnotetext{
** Note que $\alpha$ é equivalente a $\eta_{00}$ no caso de múltiplas sub-bandas.
}
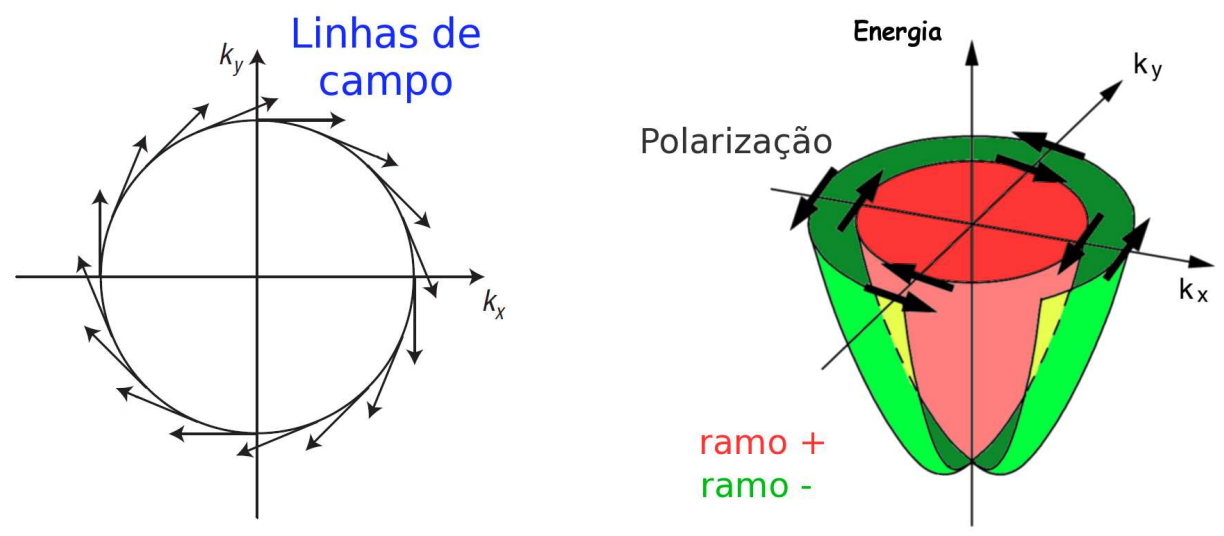

Figura 3.7 - As linhas de campo de 3.8.2, recentemente medidas por Meier et al ${ }^{29}$. A medida indireta, via campo interno, de $\alpha$ coincide até primeira casa decimal com nossos cálculos de $\eta_{00}$. À direita, os vetores polarização para o ramo $\lambda=1$ (ramo + na figura) e $\lambda=-1$ (na figura). 
hamiltoniana de Rashba pode ser escrita como

$$
H_{r}:=\alpha\left(k_{x} \sigma_{x}-k_{x} \sigma_{y}\right)=g \mu_{B} \boldsymbol{B} \cdot \boldsymbol{\sigma}
$$

em que defino o que podemos chamar de campo magnético interno

$$
\boldsymbol{B}:=\frac{\alpha}{g \mu_{B}}\left(\begin{array}{c}
k_{y} \\
-k_{x} \\
0
\end{array}\right) .
$$

As constantes $g$, fator giromagnético, e $\mu_{B}$, magneton de Bohr, apenas servem para deixar mais clara a analogia entre a hamiltoniana de Rashba e o efeito Zeeman. Note que os níveis são abertos linearmente em $k_{\|}$, assim como os níveis atômicos são abertos linearmente em função da amplitude do campo mangético. Mais ainda, só há componentes deste campo interno no plano. Se calcularmos a polarização $\langle\boldsymbol{S}\rangle$, com $\boldsymbol{S}=(\hbar / 2) \boldsymbol{\sigma}$, resultante deste campo obtemos

$$
\left\langle\lambda_{1}|\boldsymbol{S}| \lambda_{1}\right\rangle=-\frac{\lambda_{1} \hbar}{2 k_{\|}}\left(\begin{array}{c}
k_{y} \\
-k_{x} \\
0
\end{array}\right)
$$

Note que para cada ramo de energia (os diferentes valores de $\lambda_{1}$ ), a polarização aponta em um sentido. A figura 3.8 ilustra as linhas de campo de $\boldsymbol{B}$ e os vetores de polarização para cada ramo. Notemos por fim que $\boldsymbol{B}=0$ no caso simétrico, ou seja, a assimetria do potencial estrutural é importante para observarmos este campo interno.

Recentemente, esse campo foi medido por Meier et al ${ }^{29}$, usando rotação de Faraday de resolução temporal e o efeito Kerr. Como este campo interno está diretamente relacionado com a constante de acoplamento, este é um método para medição indireta do acoplamento Rashba. Comparei estas medições para o acoplamento Rashba com nossos cálculos do acoplamento intrasub-bandas com sucesso.

A seguir mostrarei como fazer algo análogo no caso de duas sub-bandas. Em vez de termos uma hamiltoniana $2 \times 2$, temos uma hamiltoniana $4 \times 4$, o que dificulta um pouco a analogia. No entanto, se um campo magnético qualquer depende da posição, por exemplo, então este campo pode ser representado por uma matriz. No caso, $\eta(z)$ pode ser encarado, a menos de fatores numéricos, como um campo magnético dependente de $z$. O acoplamento spin-órbita pode ser escrito como

$$
H_{s o}=\eta \otimes \hat{z} \cdot \boldsymbol{k}_{\|} \times \boldsymbol{\sigma}
$$


em que defino a representação matricial de $\eta(z)$

$$
\eta:=\left(\begin{array}{ll}
\eta_{00} & \eta_{01} \\
\eta_{01} & \eta_{11}
\end{array}\right)
$$

O termo $\eta \otimes \hat{z} \cdot \boldsymbol{k}_{\|} \times \boldsymbol{\sigma}$ pode ser reescrito na forma

$$
\eta \otimes \hat{z} \cdot \boldsymbol{k}_{\|} \times \boldsymbol{\sigma}=\eta \otimes \hat{z} \times \boldsymbol{k}_{\|} \cdot \boldsymbol{\sigma}
$$

Em analogia com o termo Zeeman, podemos associar $\eta \otimes \hat{z} \times k_{\|}$a um campo magnético efetivo $^{\dagger \dagger}$. Matricialmente, este campo pode ser escrito como

$$
\mathcal{B}:=\frac{1}{g \mu_{B}}\left(\begin{array}{cc}
\eta_{00} & \eta_{01} \\
\eta_{01} & \eta_{11}
\end{array}\right)\left[-k_{y} \hat{x}+k_{x} \hat{y}\right]
$$

Usando os autoestados, calculei os valores esperados deste campo magnético:

$$
\left\langle\lambda_{1} \lambda_{2}|\mathcal{B}| \lambda_{1} \lambda_{2}\right\rangle=\left(A_{\lambda_{1} \lambda_{2}}^{\boldsymbol{k}_{\|}}\right)^{2}\left(\left(B_{\lambda_{1} \lambda_{2}}^{\boldsymbol{k}_{\|}}\right)^{2} \eta_{00}+2 B_{\lambda_{1} \lambda_{2}}^{\boldsymbol{k}_{\|}} \eta_{01}+\eta_{11}\right)\left(-k_{y} \hat{x}+k_{x} \hat{y}\right) .
$$

À primeira vista salta ao olho um fato interessante: há campo magnético mesmo no caso simétrico! No caso simétrico, $\eta_{00}$ e $\eta_{11}$ se anulam, mas $\eta_{01}$ não. A equação 3.8.8 indica a existência de um campo magnético mesmo no caso simétrico, caso o segundo estado do poço quântico esteja populado. Trata-se de um campo estranho, mas podemos ver na figura 3.8 suas linhas de campo. A simetria esférica é mantida, muito embora a intensidade do campo modifique-se ligeiramente. Utilizei a notação introduzida pela equação 3.6.9.

Assim como no caso de uma sub-banda, calculei a polarização $\langle\boldsymbol{S}\rangle$ usando os estados estacionários do poço quântico:

$$
\left\langle\lambda_{1} \lambda_{2}|\boldsymbol{S}| \lambda_{1} \lambda_{2}\right\rangle=-\frac{\lambda_{1} \hbar}{2 k_{\|}}\left(\begin{array}{c}
k_{y} \\
-k_{x} \\
0
\end{array}\right)
$$

Em outras palavras, comparando à equação 3.8.3, para cada sub-banda separadamente ocorre o mesmo efeito que com uma sub-banda. Ou seja, a ideia da figura 3.8 repete-se para cada sub-banda independentemente.

Uma questão interessante é a evolução temporal dos elementos de matriz da polarização para elétrons injetados no poço quântico a partir de contatos ferromagnéticos. $O$ estado destes elétrons será uma combinação dos autoestados e talvez novos efeitos (como um campo

${ }^{\dagger \dagger}$ Embora numa estrutura $U(2) \otimes S U(2)$ em vez de $U(2) \otimes S U(1)$. 


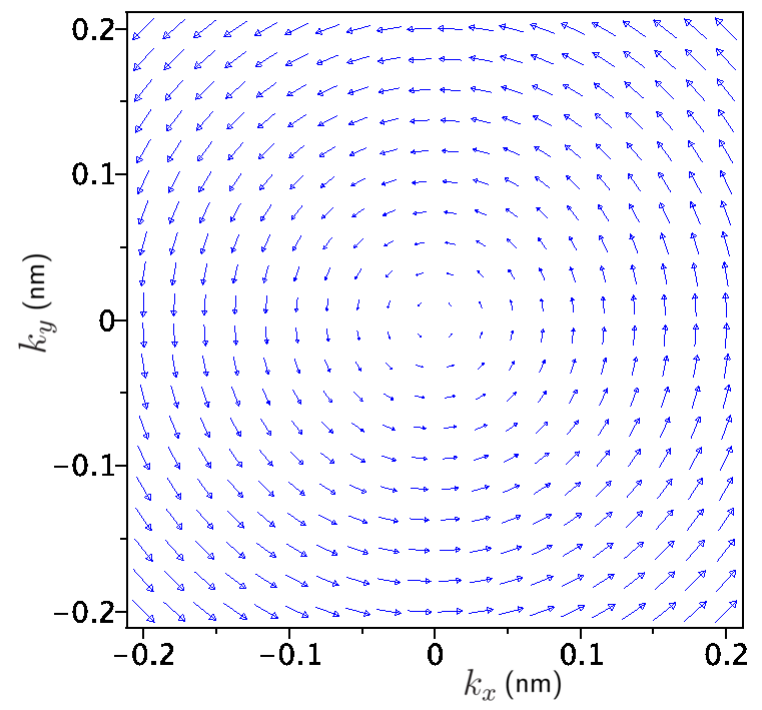

Figura 3.8 - Linhas de campo do campo magnético interno 3.8.8 calculadas para o poço quântico $A l I n S b / I n S b$ sem a aplicação de gates, i.e., no caso simétrico. Vemos novamente a simetria esférica que ocorre no caso unidimensional, muito embora a intensidade do campo modifique-se.

que gere um zitterbewegung para estes elétrons) possam ocorrer. Além disso, a adição de campos magnéticos externos pode gerar efeitos interessantes, uma vez que há diferenças entre os efeitos de campos magnéticos externos e o campo magnético interno, como observado por Ying-Tao Zhang et al ${ }^{83}$. 


\subsection{Revisão}

Adicionando o termo de Darwin e o acoplamento momento-campo elétrico, a relação de dispersão que obtemos pode ser escrita como

$$
E\left(\boldsymbol{k}_{\|}, \lambda_{1}, \lambda_{2}\right)=\widetilde{\epsilon}_{+}-\lambda_{1} \alpha_{+} k_{\|}+\lambda_{2} \Omega_{\lambda_{1}}\left(k_{\|}\right),
$$

em que defini

$$
\begin{gathered}
\alpha_{ \pm}=\frac{\eta_{11 \pm \eta_{00}}}{2}, k_{0}=\frac{H_{d e}^{01}+H_{M E C}^{01}}{\eta}, \widetilde{\epsilon}_{j}=\epsilon_{j}+H_{d e}^{j j}+H_{M E C}^{j j}, \widetilde{\epsilon}_{ \pm}=\frac{\widetilde{\epsilon}_{1} \pm \widetilde{\epsilon}_{0}}{2} \\
\Omega_{\lambda_{1}}^{2}(k)=\left(2 \widetilde{\epsilon}_{-}+\lambda_{1} \alpha_{-} k_{\|}\right)^{2}+\eta_{01}^{2}\left(k_{\|}-k_{0}\right)^{2} .
\end{gathered}
$$

Termo de Darwin estrutural:

$H_{d e}^{n m}=-\delta_{6}\left[\phi_{n}\left(a_{0}\right) \frac{d \phi_{m}}{d z}\left(a_{0}\right)+\phi_{m}\left(a_{0}\right) \frac{d \phi_{n}}{d z}\left(a_{0}\right)-\phi_{n}\left(-a_{0}\right) \frac{d \phi_{m}}{d z}\left(-a_{0}\right)-\phi_{m}\left(-a_{0}\right) \frac{d \phi_{n}}{d z}\left(-a_{0}\right)\right]$.

Acoplamento momento-campo elétrico:

$$
H_{M E C}^{n m}=-\alpha_{M} \delta_{\mu, \nu}\left[u_{n}\left(a_{0}\right) \frac{d u_{m}}{d z}\left(a_{0}\right)-u_{n}\left(-a_{0}\right) \frac{d u_{m}}{d z}\left(-a_{0}\right)\right]
$$

Os autoestados ficam

$$
\begin{gathered}
\left|\lambda_{1} \lambda_{2}\right\rangle=A_{\lambda_{1} \lambda_{2}}^{\boldsymbol{k}_{\|}}\left(A_{\lambda_{1} \lambda_{2}}^{\boldsymbol{k}_{\|}}\left|u_{0}\right\rangle+\left|u_{1}\right\rangle\right) \otimes\left|\lambda_{1}\right\rangle, \\
B_{\lambda_{1} \lambda_{2}}^{\boldsymbol{k}_{\|}}=\frac{\eta_{01}\left(k-k_{0}\right)}{\lambda_{2} \Omega_{\lambda_{1}}(k)+\left(\widetilde{\epsilon}_{-}+\lambda_{1} \alpha_{-}\right)} \quad \text { e } \quad A_{\lambda_{1} \lambda_{2}}^{\boldsymbol{k}_{\|}}=\left\{1+\left(B_{\lambda_{1} \lambda_{2}}^{\boldsymbol{k}_{\|}}\right)^{2}\right\}^{-1 / 2} .
\end{gathered}
$$

Calculei também o campo magnético interno,

$$
\mathcal{B}:=\frac{1}{g \mu_{B}}\left(\begin{array}{cc}
\eta_{00} & \eta_{01} \\
\eta_{01} & \eta_{11}
\end{array}\right)\left[-k_{y} \hat{x}+k_{x} \hat{y}\right]
$$

e seu valor médio

$$
\left\langle\lambda_{1} \lambda_{2}|\mathcal{B}| \lambda_{1} \lambda_{2}\right\rangle=\left(A_{\lambda_{1} \lambda_{2}}^{\boldsymbol{k}_{\|}}\right)^{2}\left(\left(B_{\lambda_{1} \lambda_{2}}^{\boldsymbol{k}_{\|}}\right)^{2} \eta_{00}+2 B_{\lambda_{1} \lambda_{2}}^{\boldsymbol{k}_{\|}} \eta_{01}+\eta_{11}\right)\left(-k_{y} \hat{x}+k_{x} \hat{y}\right) .
$$

A polarização média pode ser escrita como

$$
\left\langle\lambda_{1} \lambda_{2}|\boldsymbol{S}| \lambda_{1} \lambda_{2}\right\rangle=-\frac{\lambda_{1} \hbar}{2 k_{\|}}\left(\begin{array}{c}
k_{y} \\
-k_{x} \\
0
\end{array}\right) .
$$

Note que estas médias valem para todos os estados. 


\section{CAPÍTULO 4}

\section{Modelagem da Interação Elétron-Fóton}

I hear you, brave young jables, you are hungry for the rock But to learn the anciant method, secret doors you must unlock Dio, em Tenacious D - Kickapoo, 2007.

Temos em mãos um modelo efetivo de duas bandas (contando com spin) que vale, a priori, na ausência de campos eletromagnéticos. Para modelar transições ópticas na banda de condução, é necessário introduzir campos eletromagnéticos desde a equação de Schrödinger (com as correções relativísticas) e refazer tanto o modelo de Kane, como a aproximação das funções envelope. Seria então possível um modelo efetivo com interação elétron-fóton? Seria possível escrever interações efetivas elétron-fóton? De fato, mostrarei que podemos fazer isso e que há interações elétron-fóton que são induzidas pela banda de valência.

Neste capítulo, incluirei campos eletromagnéticos quantizados no procedimento desenvolvido no capítulo 2. Quantizarei o campo eletromagnético. Introduzirei o espaço de Fock* e os operadores de criação e destruição de fótons. Derivarei, por fim, os acoplamentos elétron-fóton até segunda ordem em $1 / E_{g}$.

\subsection{Hamiltoniana com interação elétron-fóton}

Do capítulo 2 até esta presente seção, trabalhamos com a hamiltoniana 2.1.1 que dividiu-se em duas contribuições, dadas pela equação 2.1.6, ao utilizarmos o teorema de Bloch (equação 2.1.2). Para inserirmos fótons no sistema, temos que incluir na hamiltoniana 2.1 .1 campos eletromagnéticos e quantizá-los em seus modos normais de vibração. E a forma de incluir

\footnotetext{
*Conhecido muitas vezes como "formalismo do número de ocupação". Veja a seção 4.2.2.
} 
estes campos na hamiltoniana é realizando a substituição mínima ${ }^{84-86}$,

$$
\boldsymbol{P} \rightarrow \boldsymbol{P}+\frac{e}{c} \boldsymbol{A}
$$

em que $\boldsymbol{A}$ é o potencial vetor e $e<0$, a carga eletrônica. Sob certas condições, conhecer $\boldsymbol{A}$ é conhecer os campos elétrico e magnético. Por hora, vamos ver as consequências da substituição mínima e, na seção 4.2, apresentarei mais detalhes sobre $\boldsymbol{A}$. Desta forma, a hamiltoniana 2.1.1 torna-se ${ }^{\dagger}$

$$
H=\frac{1}{2 m}\left(\boldsymbol{P}+\frac{e}{c} \boldsymbol{A}\right)^{2}+V(\boldsymbol{R})+\alpha \boldsymbol{\sigma} \times \nabla V(\boldsymbol{R}) \cdot\left(\boldsymbol{P}+\frac{e}{c} \boldsymbol{A}\right),
$$

com $\alpha=\hbar^{2} / 4 m^{2} c^{2}$. Como temos que calcular $(\boldsymbol{P}-\boldsymbol{A} e / c)^{2}$, será importante conhecer o comutador entre $\boldsymbol{P}$ e $\boldsymbol{A}$. Mostra-se que ${ }^{84}$

$$
[\boldsymbol{P}, \boldsymbol{A}]=\boldsymbol{P} \cdot \boldsymbol{A}-\boldsymbol{A} \cdot \boldsymbol{P} \propto \boldsymbol{\nabla} \cdot \boldsymbol{A}
$$

escolhi trabalhar no Calibre de Coulomb ${ }^{84,87}$, em que $\boldsymbol{\nabla} \cdot \boldsymbol{A}=0$. É importante deixar claro que as componentes do momento linear não comutam com as componentes do potencial vetor. De fato, o que a identidade 4.1.3, unida ao calibre de Coulomb, garante é que $\boldsymbol{P} \cdot \boldsymbol{A}=\boldsymbol{A} \cdot \boldsymbol{P}$. Portanto,

$$
\frac{1}{2 m}\left(\boldsymbol{P}-\frac{e}{c} \boldsymbol{A}\right)^{2}=\frac{P^{2}}{2 m}+\frac{e^{2}}{2 m c^{2}} A^{2}+\frac{e}{m c} \boldsymbol{P} \cdot \boldsymbol{A} .
$$

Assim, a equação 4.1 .2 pode ser reescrita na forma

$$
H=\frac{P^{2}}{2 m}+\frac{e^{2}}{2 m c^{2}} A^{2}+\frac{e}{m c} \boldsymbol{P} \cdot \boldsymbol{A}+V(\boldsymbol{R})+\alpha \boldsymbol{\sigma} \times \nabla V(\boldsymbol{R}) \cdot\left(\boldsymbol{P}+\frac{e}{c} \boldsymbol{A}\right) .
$$

Esta é a hamiltoniana com que passarei a trabalhar. No entanto, não podemos conectar ainda nenhum dos resultados obtidos no capítulo 2 até que encontremos a hamiltoniana efetiva que atua nas funções de Bloch, tal como fizemos na seção 2.1. Sem isso, os cálculos tornariam-se incoerentes.

\subsubsection{Acoplamento elétron-fóton: uma visão de $\mathrm{k} \cdot \mathrm{p}$}

O método $\mathbf{k} \cdot \mathbf{p}$ baseia-se no teorema de Bloch: já que as funções de onda, dentro do contexto apropriado $\ddagger$, podem ser reescritas como o produto $e^{i \boldsymbol{k} \cdot \boldsymbol{r}} u_{\boldsymbol{k}}(\boldsymbol{r})$, vamos então utilizar $u_{\boldsymbol{k}}(\boldsymbol{r})$ como base! A ideia é obter uma hamiltoniana análoga à da equação 2.1.1, mas com a presença do potencial vetor $\boldsymbol{A}$. Note que, na seção 2.1, descobrimos como $H$ atua sobre

†'Deixei de fora a energia do campo elétromagnético, pois nos interessa compreender puramente os estados eletrônicos. Todos os termos na equação 4.1 .5 são os que operam sobre o subespaço dos fótons.

$\ddagger$ Nos referimos à necessidade de o potencial ter alguma simetria translacional. 
funções de bloch. Fomos capazes de fatorar $e^{i k \cdot r}$ em ambos os lados da equação. Para reutilizarmos o Folding Down, precisamos fazer o mesmo com os termos que envolvem o potencial vetor.

Separando os termos que envolvem exclusivamente $\boldsymbol{A}$, a aplicação da hamiltoniana 4.1.5 sobre as funções de Bloch:

$$
\begin{aligned}
{\left[\frac{e^{2}}{2 m c^{2}} A^{2}+\frac{e}{m c} \boldsymbol{A} \cdot \boldsymbol{P}+\frac{e \alpha}{c} \boldsymbol{\sigma} \times \boldsymbol{\nabla} V(\boldsymbol{R}) \cdot \boldsymbol{A}\right] e^{i \boldsymbol{k} \cdot \boldsymbol{r}} u_{\boldsymbol{k}}(\boldsymbol{r})=} \\
\quad e^{i \boldsymbol{k} \cdot \boldsymbol{r}}\left[\frac{e^{2}}{2 m c^{2}} A^{2}+\frac{e}{m c} \boldsymbol{A} \cdot(\boldsymbol{P}+\hbar \boldsymbol{k})+\frac{e \alpha}{c} \boldsymbol{\sigma} \times \boldsymbol{\nabla V}(\boldsymbol{R}) \cdot \boldsymbol{A}\right] u_{\boldsymbol{k}}(\boldsymbol{r}) .
\end{aligned}
$$

Como é possível fatorar $e^{i k \cdot r}$ também nestes termos novos, então podemos afirmar que a hamiltoniana incluindo interação elétron-fóton pode ser reescrita na forma

$$
H^{\prime}=H_{k p}+W_{k p}(\boldsymbol{k})+H_{e f}(\boldsymbol{k}),
$$

em que $H_{k p}$ é dada por 2.1.7, $W_{k p}(\boldsymbol{k})$ é dada por 2.1.8 e surge, substituindo $\alpha$,

$$
H_{e f}(\boldsymbol{k})=\frac{e^{2}}{2 m c^{2}} A^{2}+\frac{e}{m c} \boldsymbol{A} \cdot\left\{\boldsymbol{P}+\frac{\hbar}{3 m c^{2}} \boldsymbol{\sigma} \times \boldsymbol{\nabla} V(\boldsymbol{R})+\hbar \boldsymbol{k}\right\},
$$

que representa o acoplamento elétron-fóton. A equação 4.1.8 estabelece uma hamiltoniana $\mathrm{k} \cdot \mathrm{p}$ que contém dois termos diferentes do usual $\boldsymbol{P} \cdot \boldsymbol{A}$. O termo proporcioanl a $\boldsymbol{k} \cdot \boldsymbol{A}$ decorre simplesmente do procedimento $\mathbf{k} \cdot \mathbf{p}$. Já o último termo, proporcional ao produto triplo $\sigma \times \nabla V(\boldsymbol{R})$, é conhecido na literatura como acoplamento assistido por $\operatorname{spin}^{88}$. O acoplamento assistido por spin deve-se à correção do momento canônico pela presença do potencial vetor em um termo de segunda ordem em $1 / c^{2}$ comparado com $\boldsymbol{P} \cdot \boldsymbol{A}$. Por este motivo, não incluirei este acoplamento em nossas contas seguintes, mas veremos um termo análogo surgir na equação efetiva para a banda de condução.

A seguir, realizarei um procedimento para entender melhor a natureza do potencial vetor: a segunda quantização. Gostaria de ambientar, nesta seção seguinte, nossa discussão em uma base forte de eletromagnetismo.

\subsection{Quantização do campo eletromagnético}

Utilizarei o sistema de unidades gaussiano, simplesmente por ser o mais frequentemente utilizado nas referências sobre eletromagnetismo. Da definição do potencial vetor, $\boldsymbol{B}(\boldsymbol{r}, t)=$ 
$\nabla \times \boldsymbol{A}(\boldsymbol{r}, t)$. Da lei de Ampère ${ }^{89}$,

$$
\nabla \times \boldsymbol{E}(\boldsymbol{r}, t)=-\frac{1}{c} \frac{\partial \boldsymbol{B}}{\partial t}(\boldsymbol{r}, t)=-\frac{1}{c} \frac{\partial}{\partial t} \nabla \times \boldsymbol{A}(\boldsymbol{r}, t) .
$$

Portanto, reescreverei na forma

$$
\nabla \times\left[\boldsymbol{E}(\boldsymbol{r}, t)+\frac{1}{c} \frac{\partial \boldsymbol{A}}{\partial t}(\boldsymbol{r}, t)\right]=0
$$

para tornar explícito que o vetor $\boldsymbol{E}(\boldsymbol{r}, t)+1 / c \partial \boldsymbol{A} / \partial t(\boldsymbol{r}, t)$ tem rotacional, de fato, nulo (em contrapartida com a eletroestática, em que $\nabla \times \boldsymbol{E}=0)$. Esta equação implica na existência de uma função potencial $\varphi$ que satisfaz

$$
\boldsymbol{E}(\boldsymbol{r}, t)+\frac{1}{c} \frac{\partial \boldsymbol{A}}{\partial t}(\boldsymbol{r}, t)=-\nabla \varphi(\boldsymbol{r}, t)
$$

Além disso, passarei a supor que a região em que gostaríamos de resolver os campos eletromagnéticos não há cargas (ao menos não cargas livres), o que significa $\varphi(\boldsymbol{r}, t)=0$. Com isso e com o calibre de Coulomb, o potencial vetor pode ser completamente determinado segundo a equação ${ }^{89}$

$$
\nabla^{2} \boldsymbol{A}(\boldsymbol{r}, t)-\frac{1}{c^{2}} \frac{\partial^{2} \boldsymbol{A}}{\partial t^{2}}(\boldsymbol{r}, t)=0 .
$$

A primeira quantização que faremos sobre o campo eletromagnético segue da condição de contorno,

$$
\boldsymbol{A}\left(\boldsymbol{r}+L_{j} \hat{e}_{j}, t\right)=\boldsymbol{A}(\boldsymbol{r}, t),
$$

em que $j \in\{x, y, z\}$. As quantidades $L_{j}$ definem a frequência de oscilação dos campos (por meio do comprimento de onda) ${ }^{84}$. Esta condição define por completo o problema e a solução será única quando fornecermos $\boldsymbol{A}(\boldsymbol{r}, 0)$ (condição inicial). A equação 4.2 .4 é, na verdade, um sistema de três equações diferenciais desacopladas e separáveis no tempo, o que sugere a separação

$$
\boldsymbol{A}(\boldsymbol{r}, t)=p(t) \mathcal{A}(\boldsymbol{r})
$$

Para a parte espacial, vale

$$
\nabla^{2} \mathcal{A}(\boldsymbol{r}, t)-\frac{\omega_{\boldsymbol{q}}^{2}}{c^{2}} \mathcal{A}(\boldsymbol{r}, t)=0
$$

em que $\omega_{\boldsymbol{q}}^{2}$ é a constante de separação. O vetor $\boldsymbol{q}$, implicitamente introduzido na última equação, etiqueta todos os possíveis valores para esta constante de separação. Da equação 4.2.7 fica claro que $\omega_{q}^{2}$ é a frequência de oscilação de um modo normal do campo.

Por fim, a solução da equação 4.2.4, dadas as condições 4.2.5, pode ser escrita na 
forma $84,87,89$

$$
\boldsymbol{A}(\boldsymbol{r}, t)=\sum_{\boldsymbol{q}, j}\left\{p_{\boldsymbol{q} j} \boldsymbol{\pi}_{\boldsymbol{q} j} e^{i\left(\boldsymbol{q} \cdot \boldsymbol{r}-\omega_{\boldsymbol{q} t}\right)}+c . c .\right\},
$$

em que $p_{\boldsymbol{q} j}$ depende da condição inicial e c.c. significa "complexo conjugado". Do calibre de Coulomb, segue a necessidade de $\boldsymbol{q} \cdot \boldsymbol{\pi}_{\boldsymbol{q} j}=0$ valer. O vetor $\boldsymbol{q}$ é o vetor de propagação da onda - fornece direção e sentido da propagação da onda. O vetor $\pi$, que sempre é perpendicular a $\boldsymbol{q}$, é conhecido como vetor polarização do campo eletromagnético. Como há dois possíveis vetores linearmente independentes e perpendiculares a um dado $\boldsymbol{q}$, então há duas possíveis polarizações para cada vetor de onda $\boldsymbol{q}$, i.e., $j \in\{1,2\}$. Além disso, da quantização 4.2.5, obtemos

$$
q_{j} L_{j}=2 \pi n_{j}
$$

Portanto, os $L_{j}$ definem não apenas a direção de propagação, como também o comprimento de onda e a frequência de oscilação do campo.

A energia do campo eletromagnético pode ser escrita como

$$
H_{f}=\frac{1}{4 \pi} \int d^{3} r E^{2}=\sum_{\boldsymbol{q} j} \omega_{\boldsymbol{q}} p_{\boldsymbol{q} j} p_{\boldsymbol{q} j}^{*} .
$$

E neste momento quantizaremos o campo pela segunda vez ${ }^{\S}$ podemos reescrever $p_{\boldsymbol{q} j}$ e $p_{\boldsymbol{q} j}^{*}$ como duas variáveis

$$
\begin{aligned}
Q_{\boldsymbol{q} j} & =p_{\boldsymbol{q} j}+p_{\boldsymbol{q} j}^{*}, \\
P_{\boldsymbol{q} j} & =-i \omega_{\boldsymbol{q}}\left(p_{\boldsymbol{q} j}-p_{\boldsymbol{q} j}^{*}\right) .
\end{aligned}
$$

Note que $P_{\boldsymbol{q} j}=d Q_{\boldsymbol{q} j} / d t$. Com estas novas definições, a energia do campo torna-se

$$
H=\sum_{\boldsymbol{q} j} \omega_{\boldsymbol{q}} p_{\boldsymbol{q} j} p_{\boldsymbol{q} j}^{*}=\sum_{\boldsymbol{q} j}\left(P_{\boldsymbol{q} j}^{2}-\omega_{\boldsymbol{q}} Q_{\boldsymbol{q} j}^{2}\right)
$$

Podemos impor a regra $\left[Q_{\boldsymbol{q} j}, P_{\boldsymbol{q}^{\prime} j^{\prime}}\right]=i \hbar \delta_{\boldsymbol{q}, \boldsymbol{q}^{\prime}} \delta_{j, j^{\prime}}$, o que leva necessariamente a $\left[p_{\boldsymbol{q} j}, p_{\boldsymbol{q}^{\prime} j^{\prime}}^{\dagger}\right]=$ $\frac{\hbar}{2 \omega_{\boldsymbol{q} j}} \delta_{\boldsymbol{q}, \boldsymbol{q}^{\prime}} \delta_{j, j^{\prime}}$. Para me livrar de fatores numéricos, redefini

$$
\begin{aligned}
& a_{\boldsymbol{q} j}=\left(\frac{\hbar}{2 \omega_{\boldsymbol{q} j}}\right)^{1 / 2} p_{\boldsymbol{q} j}, \\
& a_{\boldsymbol{q} j}^{\dagger}=\left(\frac{\hbar}{2 \omega_{\boldsymbol{q} j}}\right)^{1 / 2} p_{\boldsymbol{q} j}^{\dagger},
\end{aligned}
$$

\footnotetext{
§Não há nenhuma ligação com segunda quantização (que utilizaremos na seção 4.2.2).
} 
então podemos passar a utilizar os operadores $a_{\boldsymbol{q} j}$ e $a_{\boldsymbol{q} j}^{\dagger}$, cuja regra de comutação é

$$
\left[a_{\boldsymbol{q} j}, a_{\boldsymbol{q}^{\prime} j^{\prime}}^{\dagger}\right]=\delta_{\boldsymbol{q}, \boldsymbol{q}^{\prime}} \delta_{j, j^{\prime}}
$$

Nestas novas variáveis, a energia fica

$$
H=\sum_{\boldsymbol{q} j}\left(a_{\boldsymbol{q} j} a_{\boldsymbol{q} j}^{\dagger}+\frac{1}{2}\right) \hbar \omega_{\boldsymbol{q}}
$$

O operador $a_{\boldsymbol{q} j} a_{\boldsymbol{q} j}^{\dagger}$ é conhecido como o operador número de fótons, analogamente ao que se utiliza como oscilador harmônico quântico ${ }^{7}$. Por fim, o vetor potencial torna-se

$$
\boldsymbol{A}(\boldsymbol{r}, t)=\sum_{\boldsymbol{q} j} \sqrt{\frac{2 \pi \hbar c^{2}}{\omega_{\boldsymbol{q}} V}} \boldsymbol{\pi}_{\boldsymbol{q} \boldsymbol{j}}\left(a_{\boldsymbol{q} j} e^{i \boldsymbol{q} \cdot \boldsymbol{r}} e^{-i \omega_{\boldsymbol{q}} t}+a_{\boldsymbol{q} j}^{\dagger} e^{-i \boldsymbol{q} \cdot \boldsymbol{r}} e^{i \omega_{\boldsymbol{q}} t}\right)
$$

com $V$ sendo o volume em que a onda se espalha. Os operadores $a_{\boldsymbol{q} j}$ e $a_{\boldsymbol{q} j}^{\dagger}$ são operadores de criação e destruição de fótons, i.e., são operadores que alteram o número de fótons presentes no meio. Em breve, na seção 4.2.2, definiremos apropriadamente o espaço em que estes operadores atuam. Para mais detalhes, veja referências em Mecânica Quântica e/ou Teoria de Representação de Grupos ${ }^{7,84,90}$.

\subsubsection{Os diferentes acoplamentos e ordens de grandezas}

Determinado $\boldsymbol{A}$ na equação 4.2.16, escrevo $H_{e f}$ da equação 4.1 .8 com todos os operadores explícitos:

$$
\begin{array}{r}
H_{e f}(\boldsymbol{k})=\frac{e^{2}}{2 m c^{2}} A^{2}+\sqrt{\frac{2 \pi \hbar e^{2}}{V m^{2}}} \sum_{\boldsymbol{q} j} \frac{1}{\sqrt{\omega_{\boldsymbol{q}}}}\left(a_{\boldsymbol{q} j} e^{i \boldsymbol{q} \cdot \boldsymbol{r}} e^{-i \omega_{\boldsymbol{q}} t}+a_{\boldsymbol{q} j}^{\dagger} e^{-i \boldsymbol{q} \cdot \boldsymbol{r}} e^{i \omega_{\boldsymbol{q}} t}\right) \\
\boldsymbol{\pi}_{\boldsymbol{q} j} \cdot\left\{\boldsymbol{P}+\frac{\hbar}{3 m c^{2}} \boldsymbol{\sigma} \times \boldsymbol{\nabla V}(\boldsymbol{R})+\hbar \boldsymbol{k}\right\},
\end{array}
$$

Há três contribuições distintas nesta expressão: o acoplamento usual $(\boldsymbol{\pi} \cdot \boldsymbol{P})$, um acoplamento $a$ la $\mathrm{k} \cdot \mathbf{p}(\boldsymbol{\pi} \cdot \boldsymbol{k})$ e o acoplamento assistido por spin $(\boldsymbol{\pi} \cdot \boldsymbol{\sigma} \times \boldsymbol{\nabla} V(\boldsymbol{R}))^{88}$. Espera-se, naturalmente, que o acoplamento assistido por spin seja o menor, porque sua origem, o acoplamento spinórbita, é uma contribuição de em segunda ordem de $1 / c$ em relação ao termo cinético (ordem zero). Na tabela 4.1 podemos ver as ordens de grandezas de cada um destes termos, muito embora não seja possível compará-los desde já por terem unidades completamente diferentes.

Como $H_{e f}$ depende do tempo, na seção 4.8 apresentarei um procedimento usual para tratar perturbações dependentes do tempo. Primeiramente, precisamos saber quais são, exatamente, as perturbações. Para tal, realizarei o Folding Down na hamiltoniana com acoplamento $\boldsymbol{P} \cdot \boldsymbol{A}$. 
Tabela 4.1 - Tabela com os valores numéricos coeficientes e as unidades de cada um dos acoplamentos dados em $H_{e f}$ (equação 4.2.17). Estão presentes o acoplamento usual $(\boldsymbol{\pi} \cdot \boldsymbol{P})$, um acoplamento a la $\mathbf{k} \cdot \mathbf{p}(\boldsymbol{\pi} \cdot \boldsymbol{k})$ e o acoplamento assistido por spin $(\boldsymbol{\pi} \cdot \boldsymbol{\sigma} \times \nabla V(\boldsymbol{R}))$.

\begin{tabular}{ccc}
\hline Fonte & Coeficiente & Unidade \\
\hline $\boldsymbol{\pi} \cdot \boldsymbol{P}$ & $1.812 \times 10^{-15}$ & $\mathrm{Cm}^{2} \mathrm{~s}^{-3 / 2} \mathrm{eV}^{-1 / 2}$ \\
$\boldsymbol{\pi} \cdot \boldsymbol{k}$ & $1.193 \times 10^{-30}$ & $\mathrm{Cm}^{3} \mathrm{eV}^{1 / 2} \mathrm{~s}^{-1 / 2}$ \\
$\boldsymbol{\pi} \cdot \boldsymbol{\sigma} \times \boldsymbol{\nabla} V(\boldsymbol{R})$ & $3.755 \times 10^{-26}$ & $\mathrm{Cm}^{2} \mathrm{~s}^{-1} \mathrm{eV}^{-1 / 2}$ \\
\hline
\end{tabular}

\subsubsection{Base com número de fótons}

Com a segunda quantização, é possível utilizar uma base especial para os estados de fóton: o número de ocupação. Denotarei por $\left|n_{\boldsymbol{q}, \lambda}\right\rangle$ o estado em que há $n$ fótons, todos com vetor de onda $\boldsymbol{q}$ e polarização $\pi_{\boldsymbol{q} \lambda}$ 『. Uma extensão natural desta notação é o uso de produtos diretos destes estados para gerar um "ambiente de fótons". O estado

$$
\left|n_{\boldsymbol{q}, \lambda}, m_{\boldsymbol{q}^{\prime}, \lambda^{\prime}}, \ldots\right\rangle:=\left|n_{\boldsymbol{q}, \lambda}\right\rangle \otimes\left|m_{\boldsymbol{q}^{\prime}, \lambda^{\prime}}\right\rangle \otimes \ldots
$$

signfica que há $n$ fótons no estado $\left|n_{\boldsymbol{q}, \lambda}\right\rangle, m$ no estado $\left|m_{\boldsymbol{q}^{\prime}, \lambda^{\prime}}\right\rangle$, etc. Costuma-se chamar de vácuo o estado em que não há nenhum fóton, o que denotarei por $|0\rangle$. O espaço gerado por todos estes estados é um tipo especial de espaço vetorial: Espaço de Fock, uma extensão do espaço de Hilbert usualmente construído para trabalhar com muitas partículas.

A ideia por trás desta base está na forma como os operadores de criação e destruição atuam nela,

$$
\begin{aligned}
a_{\boldsymbol{q} j}\left|n_{\boldsymbol{q} j}\right\rangle & =\sqrt{n}\left|(n-1)_{\boldsymbol{q} j}\right\rangle \\
a_{\boldsymbol{q}^{j}}^{\dagger}\left|n_{\boldsymbol{q} j}\right\rangle & =\sqrt{n+1}\left|(n+1)_{\boldsymbol{q} j}\right\rangle \\
a_{\boldsymbol{q}^{\prime} j^{\prime}}\left|n_{\boldsymbol{q} j}\right\rangle & =0 \\
a_{\boldsymbol{q}^{\prime} j^{\prime}}^{\dagger}\left|n_{\boldsymbol{q} j}\right\rangle & =\sqrt{1}\left|n_{\boldsymbol{q} j}, 1_{\boldsymbol{q}^{\prime} j^{\prime}}\right\rangle .
\end{aligned}
$$

Note que o vácuo $|0\rangle$ é autovetor de todos os operadores de destruição. Estes são os únicos operadores que atuam sobre os estados de fótons. Todos os operadores com que trabalhamos até o momento não alteram o estado do campo eletromagnético (e, consequentemente, dos fótons).

Este espaço que construímos descreve os estados possíveis dos fótons, mas nosso sistema

TVale lembrar que fótons são bósons, e por isso não restrição sobre $n$. 
é constituído de elétrons e fótons. O estado do sistema será o produto direto com o estado de fótons e os estados eletrônicos. Portanto, para me referir ao sistema com um elétron de condução com estado $\left|\lambda_{1} \lambda_{2}\right\rangle$ e um feixe de fótons no estado $\left|n_{\boldsymbol{q}, \lambda}, m_{\boldsymbol{q}^{\prime}, \lambda^{\prime}}, \ldots\right\rangle$, utilizarei a notação

$$
\left|\lambda_{1} \lambda_{2} ; n_{\boldsymbol{q} j}, m_{\boldsymbol{q}^{\prime} j^{\prime}}, \ldots\right\rangle:=\left|\lambda_{1} \lambda_{2}\right\rangle \otimes\left|n_{\boldsymbol{q} j}, m_{\boldsymbol{q}^{\prime} j^{\prime}}, \ldots\right\rangle
$$

Na equação 4.2.16 há operadores mistos, i.e., operadores que atuam tanto em estados de fóton como em estados eletrônicos. Os elementos de matriz para operadores mistos $H=H_{e} H_{f}$, em que $H_{e}$ atua na parte eletrônica e $H_{f}$ atua nos estados de fótons, são dados por

$$
\begin{aligned}
\left\langle\lambda_{1} \lambda_{2} ; n_{\boldsymbol{q} j}, m_{\boldsymbol{q}^{\prime} j^{\prime}}, \ldots\right| H & \left|\lambda_{1}^{\prime} \lambda_{2}^{\prime} ; n_{\boldsymbol{q}_{j}}^{\prime}, m_{\boldsymbol{q}^{\prime} j^{\prime}}^{\prime}, \ldots\right\rangle= \\
& \left\langle\lambda_{1} \lambda_{2}\left|H_{e}\right| \lambda_{1}^{\prime} \lambda_{2}^{\prime}\right\rangle\left\langle n_{\boldsymbol{q} j}, m_{\boldsymbol{q}^{\prime} j^{\prime}}, \ldots\left|H_{f}\right| n_{\boldsymbol{q} j}^{\prime}, m_{\boldsymbol{q}^{\prime} j^{\prime}}^{\prime}, \ldots\right\rangle,
\end{aligned}
$$

Por exemplo, os processos de absorção e emissão serão tratados usando $H_{f} \propto a_{\boldsymbol{q} \lambda}$ e $H_{f} \propto a_{\boldsymbol{q} \lambda}^{\dagger}$, respectivamente.

Os estados de fótons dependem da polarização $\pi$ do campo de radiação. Dado um vetor de onda $\boldsymbol{q}$, há duas possíveis polarizações admissíveis, que formam um plano perpedicular a $\boldsymbol{q}$. Mostrarei, no capítulo 5, que a polarização contribui de duas formas distintas: a polarização in plane (em plano), perpendicular ao eixo de crescimento do poço quântico, e a polarização perpendicular, que é paralela ao eixo de crescimento. A figura 4.1 ilustra a polarização em plano e perpendicular geometricamente. Esta diferenciação só ocorre por causa da quebra de simetria translacional, no caso de um ponto quântico teríamos contribuições análogas induzidas por todas as direções de polarização. Por isso, podemos afirmar que esta diferenciação decorre

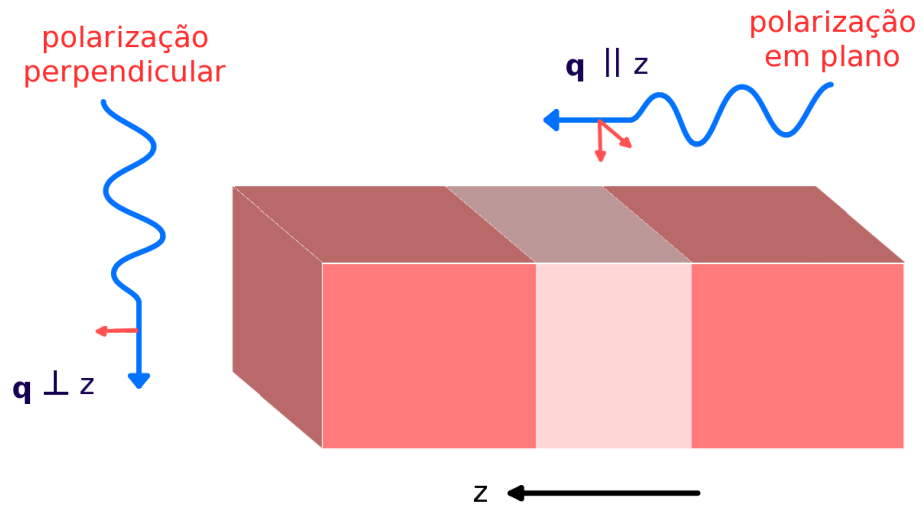

Figura 4.1 - A polarização (setas em vermelho), sempre perpendicular ao vetor de onda $\boldsymbol{q}$, induz transições ópticas de duas formas distintas: parte advém da componente paralela ao gás bidimensional confinado no poço quântico, outra contribuição vem da componente paralela ao eixo de crescimento do poço quântico. No capítulo 5 ficará clara essa distinção, mas podemos adiantar que isso reflete diretamente a quebra de simetria na direção de crescimento. 


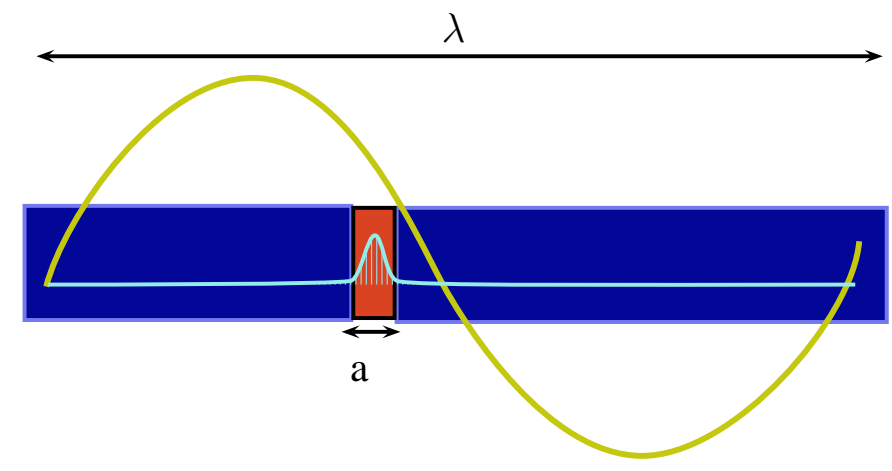

Figura 4.2 - O poço quântico confina os elétrons da banda de condução numa região de largura de alguns nanometros. Esse confinamento confina o elétron na região central do poço quântico. Se o comprimento de onda for muito grande comparado à largura do poço, então a onda eletromagnética é praticamente uma constante na região de confinamento. Portanto, num dado instante de tempo, o elétron quase não experimenta a oscilação espacial do campo eletromagnético.

diretamente da quebra de simetria no eixo de crescimento.

Notemos por fim que $A^{2}$ é proporcional a produtos de operadores de criação e destruição. Portanto, ocorrem dois processos (duas absorções, duas emissões ou tanto uma emissão quanto uma absorção). Estes processos são conhecidos como processos de dois fótons.

\subsection{Aproximação de dipolo elétrico}

Na maioria das ligas binárias de poços quânticos da família $I I I-V$, os elétrons nas subbandas de condução estão confinados em regiões nanométricas. Se estivermos interessados em feixes laser com comprimento de onda da ordem de $100 \mathrm{~nm}$ (menos da metade do comprimento de onda do ultravioleta, $350 \mathrm{~nm} \rightarrow 450 \mathrm{~nm}$ ), então o comprimento de onda será cem vezes maior que o espaço em que o elétron se confina. Como o espaçamento dos níveis energéticos das sub-bandas é da ordem de algumas dezenas de meV, deve-se esperar que lasers com frequências próximas ao do vermelho ou infra-vermelho excitem transições eletrônicas. Estas frequências estão associadas a comprimentos de onda tipicamente seis ou sete vezes maiores que $100 \mathrm{~nm}$, o que significa que o espaço em que o elétron pode se mover representa uma porção menor ainda do comprimento de onda. Podemos visualizar isso pictoricamente na figura 4.2 .

Sendo $\lambda$ o comprimento de onda desse feixe, $q=2 \pi / \lambda$ é o módulo do vetor de onda. A parte dependente do tempo na expressão 4.2.16 para o potencial vetor depende de $e^{ \pm i q \cdot \boldsymbol{r}}$. Como todos os acoplamentos dependem do potencial vetor, esta dependência temporal é 
comum a todas as diferentes contribuições para o acoplamento elétron-fóton. O argumento desta exponencial pode ser escrito como

$$
\boldsymbol{q} \cdot \boldsymbol{r}=q r \cos (\theta)=\frac{2 \pi r}{\lambda} \cos (\theta)
$$

Seguindo o viés que essas considerações anteriores sugerem, é natural esperar que $r / \lambda \ll 1$, e por isso podemos propor a aproximação

$$
e^{i \boldsymbol{q} \cdot \boldsymbol{r}}=1+\mathcal{O}(q)
$$

Esta aproximação é conhecida como aproximação de dipolo elétrico ${ }^{86}$.

\subsection{Aproximação das Funções Envelope com Campo de Radiação}

Temos uma nova equação de Schrödinger, com hamiltoniana dada na equação 4.1.7, e gostaríamos de tratá-la com as aproximações das funções envelope. Há termos que dependem do potencial vetor além de operadores que atuam sobre os estados eletrônicos, como é o caso de $\boldsymbol{P} \cdot \boldsymbol{A}$. Refarei os passos da seção 2.3.2, usando esta nova hamiltoniana. Isolarei o efeito do campo de radiação e buscarei uma equação para as funções envelope seguindo a mesma linha de raciocínio da seção 2.3.2.

É importante ressaltar que diversos grupos preferem trabalhar ainda no esquema de oito bandas, como Yang et $a^{91}{ }^{91}$ e Burt ${ }^{92}$. Isso exige um esforço computacional muito maior. Apesar de serem conceitualmente diferentes, ambas as abordagens fornecem os mesmos resultados ainda mais porque efetivamente ambas são como irmãs gêmeas.

Recomeçarei, enfim, o tratamento da seção 2.3 .2 com a hamiltoniana

$$
H|\Psi\rangle=\left[H_{0}+\phi(\boldsymbol{R})+\frac{e}{m c} \boldsymbol{P} \cdot \boldsymbol{A}+\frac{e^{2}}{2 m c^{2}} A^{2}\right]|\Psi\rangle=E|\Psi\rangle,
$$

em que $\phi(\boldsymbol{R})$ fará o papel do potencial que varia muito lentamente e uma base $\{|j\rangle, j \in \mathbb{N}\}$ tal que

$$
H_{0}|j\rangle=\epsilon_{j}|j\rangle
$$

Como ansatz, proponho o estado

$$
|\Psi\rangle=\sum_{n} F_{n}(\boldsymbol{R})|n\rangle
$$

Exatamente como feito na seção 2.3.2, gostaria de determinar uma equação efetiva para as 
funções envelope $F_{n}(\boldsymbol{R})$. Usando $4.4 .3 \mathrm{em} 4.4 .1$, vale

$$
\sum_{n}\left[H_{0}+\frac{e}{m c} \boldsymbol{P} \cdot \boldsymbol{A}\right] F_{n}(\boldsymbol{R})|n\rangle=\left(E-\phi(\boldsymbol{R})-\frac{e^{2}}{2 m c^{2}} A^{2}\right) \sum_{n} F_{n}(\boldsymbol{R})|n\rangle .
$$

Multiplicando a equação 4.4 .4 pela esquerda por $\langle j| F_{j}^{\dagger}(\boldsymbol{r})$,

$$
\begin{aligned}
\left\langle j\left|F_{j}^{\dagger}(\boldsymbol{r}) H\right| \Psi\right\rangle & =\sum_{n}\left\langle j\left|F_{j}^{\dagger}(\boldsymbol{r})\left[H_{0}+\frac{e}{m c} \boldsymbol{P} \cdot \boldsymbol{A}\right] F_{n}(\boldsymbol{R})\right| n\right\rangle= \\
& \sum_{n}\left[\left\langle j\left|F_{j}^{\dagger}(\boldsymbol{r}) H_{0} F_{n}(\boldsymbol{R})\right| n\right\rangle+\frac{e}{m c}\left\langle j\left|F_{j}^{\dagger}(\boldsymbol{r}) \boldsymbol{P} \cdot \boldsymbol{A} F_{n}(\boldsymbol{R})\right| n\right\rangle\right] .
\end{aligned}
$$

O termo que surge do acoplamento elétron-fóton pode ser simplifcado como

$$
\begin{aligned}
\left\langle j\left|F_{j}^{\dagger}(\boldsymbol{r}) \boldsymbol{P} \cdot \boldsymbol{A} F_{n}(\boldsymbol{R})\right| n\right\rangle=\int d^{3} r u_{j}^{*}(\boldsymbol{r}) u_{n}(\boldsymbol{r}) f_{j}^{*}(\boldsymbol{r}) \boldsymbol{A} \cdot\left\{-i \hbar \nabla f_{n}(\boldsymbol{r})\right\}+ \\
\qquad d^{3} r f_{j}^{*}(\boldsymbol{r}) f_{n}(\boldsymbol{r}) u_{j}^{*}(\boldsymbol{r}) \boldsymbol{A} \cdot\left\{-i \hbar \nabla u_{n}(\boldsymbol{r})\right\} .
\end{aligned}
$$

O potencial vetor está sob aproximação de dipolo elétrico, em que simplesmente não há dependência espacial alguma. Portanto, para todos os efeitos o potencial vetor é como uma funçãoll que varia lentamente, mas com três componentes. Desta forma, podemos usufruir da separação das integrais na a equação 4.4.6, de que obtemos

$$
\begin{aligned}
\left\langle j\left|F_{j}^{\dagger}(\boldsymbol{r}) \boldsymbol{P} \cdot \boldsymbol{A} F_{n}(\boldsymbol{R})\right| n\right\rangle=-i \hbar \delta_{j, n} \int d^{3} r f_{j}^{*}(\boldsymbol{r}) \boldsymbol{A} \cdot \nabla f_{n}(\boldsymbol{r})+ \\
\qquad d^{3} r f_{j}^{*}(\boldsymbol{r}) f_{n}(\boldsymbol{r}) \boldsymbol{A} \cdot\langle j|\boldsymbol{P}| n\rangle .
\end{aligned}
$$

Os outros termos ficam da mesma forma como desenvolvemos na seção 2.3.2: primeiramente faço a comutação $H_{0}$ com as funções envelope e reescrevo na forma,

$$
\begin{array}{r}
\left\langle j\left|F_{j}^{\dagger}(\boldsymbol{r}) H_{0} F_{n}(\boldsymbol{R})\right| n\right\rangle=\int \frac{d^{3}}{\Omega} \delta_{j, n}\left\{\epsilon_{n} f_{j}^{*}(\boldsymbol{r}) f_{n}(\boldsymbol{r})-\frac{\hbar^{2}}{2 m} f_{j}^{*}(\boldsymbol{r}) \nabla^{2} f_{n}(\boldsymbol{r})\right\}- \\
i \frac{\hbar}{m} \int \frac{d^{3}}{\Omega} f_{j}^{*}(\boldsymbol{r}) \nabla f_{n}(\boldsymbol{r}) \cdot\langle j|\boldsymbol{P}| n\rangle .
\end{array}
$$

Note como os termos da equação 4.4 .7 se parecem com a equação 4.4.8. Para reuni-los, os reagrupei da seguinte forma:

$$
\begin{array}{r}
\left\langle j\left|F_{j}^{\dagger}(\boldsymbol{r}) H_{0}+\frac{e}{m c} \boldsymbol{A} \cdot \boldsymbol{P} F_{n}(\boldsymbol{R})\right| n\right\rangle=\int \frac{d^{3}}{\Omega} \delta_{j, n}\left\{\epsilon_{n} f_{j}^{\dagger}(\boldsymbol{r}) f_{n}(\boldsymbol{r})-\frac{\hbar^{2}}{2 m} f_{j}^{*}(\boldsymbol{r}) \nabla^{2} f_{n}(r)-\right. \\
\left.i \frac{\hbar e}{m c} f_{j}^{*}(\boldsymbol{r}) \boldsymbol{A} \cdot \nabla f_{n}(\boldsymbol{r})\right\}+\int \frac{d^{3}}{\Omega} f_{j}^{*}(\boldsymbol{r})\left[-i \frac{\hbar}{m} \nabla+\frac{e}{m c} \boldsymbol{A}\right] f_{n}(\boldsymbol{r}) \cdot\langle j|\boldsymbol{P}| n\rangle \cdot \quad \text { (4.4.9) }
\end{array}
$$

॥Ou camop vetorial, para ser mais específico. 
Usando esta última igualdade na equação 4.4.4, obtive

$$
\begin{gathered}
\sum_{n} \int \frac{d^{3}}{\Omega} \delta_{j, n}\left\{\epsilon_{n} f_{j}^{*}(\boldsymbol{r}) f_{n}(\boldsymbol{r})-\frac{\hbar^{2}}{2 m} f_{j}^{*}(\boldsymbol{r}) \nabla^{2} f_{n}(R)-i \frac{\hbar e}{m c} f_{j}^{*}(\boldsymbol{r}) \boldsymbol{A} \cdot \nabla f_{n}(\boldsymbol{r})\right\}+ \\
\sum_{n} \int \frac{d^{3}}{\Omega} f_{j}^{*}(\boldsymbol{r})\left[-i \frac{\hbar}{m} \nabla+\frac{e}{m c} \boldsymbol{A}\right] F_{n}(\boldsymbol{r}) \cdot\langle j|\boldsymbol{P}| n\rangle= \\
\sum_{n} \int \frac{d^{3}}{\Omega} f_{j}^{*}(\boldsymbol{r})\left(E-\phi(\boldsymbol{R})-\frac{e^{2}}{2 m c^{2}} A^{2}\right) f_{n}(\boldsymbol{r}) .
\end{gathered}
$$

Para completar quadrados uni o termo proporcional a $A^{2}$ do lado direito com os termos cinético e $\boldsymbol{P} \cdot \boldsymbol{A}$,

$$
\begin{aligned}
\sum_{n} \int \frac{d^{3}}{\Omega}\{- & \frac{\hbar^{2}}{2 m} f_{j}^{*}(\boldsymbol{r}) \nabla^{2} f_{n}(R)- \\
\left.i \frac{\hbar e}{m c} f_{j}^{*}(\boldsymbol{r}) \boldsymbol{A} \cdot \nabla f_{n}(\boldsymbol{r})+\frac{e^{2}}{2 m c^{2}} A^{2}\right\} & =\frac{1}{2 m} \sum_{n} \int \frac{d^{3}}{\Omega} f_{j}^{*}(\boldsymbol{r}) \Pi^{2} f_{n},
\end{aligned}
$$

em que obtemos novamente o momento canônico $\Pi=\boldsymbol{P}+\frac{e}{c} \boldsymbol{A}$. Note que o mesmo momento $\Pi$ está presente na expressão entre colchetes da equação 4.4.10. Com isso, fecho novamente a equação para as funções envelope apenas usando o momento canônico,

$$
\begin{aligned}
\int \frac{d^{3}}{\Omega} \delta_{j, n}\left\{\left(\epsilon_{n}-E+\phi(r)\right) f_{j}^{*}(\boldsymbol{r}) f_{n}(\boldsymbol{R})-\frac{1}{2 m} f_{j}^{*}(\boldsymbol{r}) \Pi^{2} f_{n}(\boldsymbol{r})\right\}+ & \\
& \frac{1}{m} F_{j}^{\dagger}(\boldsymbol{r}) \Pi F_{n}(R) \cdot\langle j|\boldsymbol{P}| n\rangle .
\end{aligned}
$$

Esta última equação é exatamente igual à 2.3.22 quando $\boldsymbol{A}=\mathbf{0}$. Naturalmente, pode também ser mapeada na equação 2.1 .11 do método $\mathbf{k} \cdot \mathbf{p}$ se identificado $k \rightarrow-i \nabla$.

Podemos concluir, portanto, que adicionar um campo de radiação implica na substituição mínima na equação das funções envelope. E como deve então ficar a equação efetiva para a banda de condução? Mostrarei a seguir o que a substituição mínima implica na equação efetiva da banda de condução.

\subsection{Equação efetiva para banda de condução com campo de radiação}

Na seção anterior, mostrei que, na presença de um campo magnético, a única diferença na hamiltoniana de Kane (equação 2.4.5) é a substituição mínima. Portanto, na presença de um campo magnético, trabalharei com um modelo de Kane com Campo de Radiação, cuja 
hamiltoniana é dada por

$$
H=\left(\begin{array}{ccc}
E_{6} \mathbb{1}_{2 \times 2} & \sqrt{\frac{2}{3}} \mathcal{P} \boldsymbol{T} \cdot\left(\boldsymbol{k}+\frac{e}{\hbar c} \boldsymbol{A}\right) & -\frac{\mathcal{P}}{\sqrt{3}} \boldsymbol{\sigma} \cdot\left(\boldsymbol{k}+\frac{e}{\hbar c} \boldsymbol{A}\right) \\
\sqrt{\frac{2}{3}} \mathcal{P} \boldsymbol{T}^{\dagger} \cdot\left(\boldsymbol{k}+\frac{e}{\hbar c} \boldsymbol{A}\right) & E_{8} \mathbb{1}_{4 \times 4} & 0 \\
-\frac{\mathcal{P}}{\sqrt{3}} \boldsymbol{\sigma} \cdot\left(\boldsymbol{k}+\frac{e}{\hbar c} \boldsymbol{A}\right) & 0 & E_{7} \mathbb{1}_{2 \times 2}
\end{array}\right)
$$

em que as matrizes $\boldsymbol{T}$ já são nossas bem conhecidas. Compensa relembrar que as diagonais,

$$
\begin{gathered}
E_{6}=V+h_{6}+\frac{\hbar^{2}}{2 m}\left(\boldsymbol{k}+\frac{e}{\hbar c} \boldsymbol{A}\right)^{2}, \\
E_{8}=V-h_{8}-E_{g}+\frac{\hbar^{2}}{2 m}\left(\boldsymbol{k}+\frac{e}{\hbar c} \boldsymbol{A}\right)^{2}, \\
E_{7}=V-h_{7}-E_{g}-\Delta_{g}+\frac{\hbar^{2}}{2 m}\left(\boldsymbol{k}+\frac{e}{\hbar c} \boldsymbol{A}\right)^{2},
\end{gathered}
$$

formam o perfil estrutural e que, desta vez, dependem do potencial vetor.

É importante deixar claro que a base com que estou trabalhando não foi alterada: continuam as oito funções envelope. Estas oito dividem-se em duas para banda de condução, denotadas por $\psi$; quatro para buracos leves e pesados, denotados por $\phi$; e duas para buracos de split-off, denotados por $\chi$. As equações para as bandas de valência tornam-se, na presença do campo de radiação,

$$
\begin{gathered}
\phi=\frac{1}{\epsilon-E_{8}} \sqrt{\frac{2}{3}} \mathcal{P} \boldsymbol{T}^{\dagger} \cdot\left(\boldsymbol{k}+\frac{e}{\hbar c} \boldsymbol{A}\right) \psi, \\
\chi=\frac{-1}{\epsilon-E_{7}} \frac{\mathcal{P}}{\sqrt{3}} \boldsymbol{\sigma} \cdot\left(\boldsymbol{k}+\frac{e}{\hbar c} \boldsymbol{A}\right) \psi .
\end{gathered}
$$

Similarmente, a equação para a banda de condução torna-se

$$
\begin{aligned}
\frac{\mathcal{P}^{2}}{3}\left[\boldsymbol{T} \cdot\left(\boldsymbol{k}+\frac{e}{\hbar c} \boldsymbol{A}\right) \frac{2}{\epsilon-E_{8}}\right. & \boldsymbol{T}^{\dagger} \cdot\left(\boldsymbol{k}+\frac{e}{\hbar c} \boldsymbol{A}\right)+ \\
& \left.\boldsymbol{\sigma} \cdot\left(\boldsymbol{k}+\frac{e}{\hbar c} \boldsymbol{A}\right) \frac{1}{\epsilon-E_{7}} \boldsymbol{\sigma} \cdot\left(\boldsymbol{k}+\frac{e}{\hbar c} \boldsymbol{A}\right)\right] \psi=\left(\epsilon-E_{6}\right) \psi .
\end{aligned}
$$

Dividirei a hamiltoniana efetiva da equação 4.5.4 em duas partes: termos que não contêm qualquer informação do campo de radiação, digamos $\mathcal{H}_{0}$, e termos que contém o potencial vetor $\boldsymbol{A}$, digamos $\mathcal{H}_{a}$, ou seja,

$$
\mathcal{H} \psi=\left(\mathcal{H}_{0}+\frac{\mathcal{P}^{2}}{3} \mathcal{H}_{a}\right) \psi=\left(\epsilon-E_{6}\right) \psi,
$$

em que o fator comum $\mathcal{P}^{2} / 3$ foi separado simplesmente para facilitar as expressões que estão por vir. Ao refazer os passos das seções 2.7, 2.7 .1 e 2.7 .2 com $\mathcal{H}$, então $\mathcal{H}_{0}$ se tornará exatamente a hamiltoniana 2.7.27. Consequentemente, $\left(\mathcal{P}^{2} / 3\right) \mathcal{H}_{a}$ será a interação efetiva entre o elétron e os fótons. No entanto adianto ao leitor que, como resultado, as interações 
elétrons de condução-fótons não vêm da substituição mínima direto na equação efetiva para elétrons de condução, como se esperaria. Um descuido como esse levaria a termos que nem ao menos hermitianos são ${ }^{81,93}$. Há, no entanto, um pequeno detalhe que, a tempo, esclarecerei.

Simplificarei primeiramente o lado esquerdo da equação 4.5.4. Os produtos podem ser expandidos associativamente, ou seja,

$$
\begin{aligned}
\boldsymbol{T} \cdot\left(\boldsymbol{k}+\frac{e}{\hbar c} \boldsymbol{A}\right) \frac{1}{\epsilon-E_{8}} \boldsymbol{T}^{\dagger} \cdot\left(\boldsymbol{k}+\frac{e}{\hbar c} \boldsymbol{A}\right)=\boldsymbol{T} \cdot \boldsymbol{k} \frac{1}{\epsilon-E_{8}} \boldsymbol{T}^{\dagger} \cdot \boldsymbol{k}+ \\
\quad \frac{e}{\hbar c}\left\{\boldsymbol{T} \cdot \boldsymbol{A} \frac{1}{\epsilon-E_{8}} \boldsymbol{T}^{\dagger} \cdot \boldsymbol{k}+\boldsymbol{T} \cdot \boldsymbol{k} \frac{1}{\epsilon-E_{8}} \boldsymbol{T}^{\dagger} \cdot \boldsymbol{A}\right\}+\left(\frac{e}{\hbar c}\right)^{2} \boldsymbol{T} \cdot \boldsymbol{A} \frac{1}{\epsilon-E_{8}} \boldsymbol{T}^{\dagger} \cdot \boldsymbol{A} .
\end{aligned}
$$

O primeiro termo do lado direito desta última equação é simplesmente parte da equação efetiva que já tinhamos, i.e., será mantido em $\mathcal{H}_{0}$; os outros termos vão para $\mathcal{H}_{a}$. A troca de $\boldsymbol{T}$ por $\boldsymbol{\sigma}$ e $E_{8}$ por $E_{7}$ leva à expansão para os operadores da segunda linha da equação 4.5.4. Explicitamente,

$$
\begin{aligned}
\boldsymbol{\sigma} \cdot\left(\boldsymbol{k}+\frac{e}{\hbar c} \boldsymbol{A}\right) \frac{1}{\epsilon-E_{7}} \boldsymbol{\sigma} \cdot\left(\boldsymbol{k}+\frac{e}{\hbar c} \boldsymbol{A}\right)=\boldsymbol{\sigma} \cdot \boldsymbol{k} \frac{1}{\epsilon-E_{7}} \boldsymbol{\sigma} \cdot \boldsymbol{k}+ \\
\frac{e}{\hbar c}\left\{\boldsymbol{\sigma} \cdot \boldsymbol{A} \frac{1}{\epsilon-E_{7}} \boldsymbol{\sigma} \cdot \boldsymbol{k}+\boldsymbol{\sigma} \cdot \boldsymbol{k} \frac{1}{\epsilon-E_{7}} \boldsymbol{\sigma} \cdot \boldsymbol{A}\right\}+\left(\frac{e}{\hbar c}\right)^{2} \boldsymbol{\sigma} \cdot \boldsymbol{A} \frac{1}{\epsilon-E_{7}} \boldsymbol{\sigma} \cdot \boldsymbol{A} .
\end{aligned}
$$

Novamente o primeiro termo do lado direito vai para $\mathcal{H}_{0}$ e os outros, para $\mathcal{H}_{a}$.

Além destes termos explícitos, existe uma fonte de acoplamento elétron-fóton que pode ser extraído do lado direito da equação 4.5 .4 , pois $E_{6}$ contém termos com $\boldsymbol{A}$ (veja equação 4.5.2). Escolhi separá-la da seguinte forma:

$$
\begin{aligned}
&\left(\epsilon-E_{6}\right) \psi=\left[\epsilon-V-h_{6}-\frac{\hbar^{2}}{2 m}\left(\boldsymbol{k}+\frac{e}{\hbar c} \boldsymbol{A}\right)^{2}\right] \psi= \\
&\left(\epsilon-V-h_{6}-\frac{\hbar^{2}}{2 m} k^{2}\right) \psi-\left(\frac{\hbar e}{m c} \boldsymbol{A} \cdot \boldsymbol{k}+\frac{e^{2}}{2 m c^{2}} A^{2}\right) \psi
\end{aligned}
$$

Estão presentes nesta última equação os acoplamentos usuais $\boldsymbol{P} \cdot \boldsymbol{A}$ e $A^{2}$. Algo extremamente interessante é que estes acoplamentos são os únicos oriundos da própria banda de condução. Todos os outros acoplamentos que obterei advém do acoplamento entre as bandas de valência e condução. A massa que aparece neste acoplamento é a massa eletrônica (que vem diretamente da equação de Pauli). As correções da massa efetiva surgirão naturalmente, i.e., alguns termos similares aos da equação 4.5.8 farão o papel de "correção a massa efetiva eletrônica". Essa correção da massa também é induzida pelas bandas de valência 
Reunindo todos os termos que dependem do potencial vetor,

$$
\begin{aligned}
\mathcal{H}_{a}=\frac{e}{m c} \boldsymbol{P} \cdot \boldsymbol{A}+\frac{e^{2}}{2 m c^{2}} A^{2}+ & \\
\frac{e}{\hbar c}\left\{\boldsymbol{T} \cdot \boldsymbol{A} \frac{2}{\epsilon-E_{8}} \boldsymbol{T}^{\dagger} \cdot \boldsymbol{k}+\right. & \left.\boldsymbol{T} \cdot \boldsymbol{k} \frac{2}{\epsilon-E_{8}} \boldsymbol{T}^{\dagger} \cdot \boldsymbol{A}+\boldsymbol{\sigma} \cdot \boldsymbol{A} \frac{1}{\epsilon-E_{7}} \boldsymbol{\sigma} \cdot \boldsymbol{k}+\boldsymbol{\sigma} \cdot \boldsymbol{k} \frac{1}{\epsilon-E_{7}} \boldsymbol{\sigma} \cdot \boldsymbol{A}\right\} \\
& \quad\left(\frac{e}{\hbar c}\right)^{2}\left\{\boldsymbol{T} \cdot \boldsymbol{A} \frac{2}{\epsilon-E_{8}} \boldsymbol{T}^{\dagger} \cdot \boldsymbol{A}+\boldsymbol{\sigma} \cdot \boldsymbol{A} \frac{1}{\epsilon-E_{7}} \boldsymbol{\sigma} \cdot \boldsymbol{A}\right\} .
\end{aligned}
$$

Esta é a parte da hamiltoniana efetiva que acopla os elétrons de condução com o campo de radiação. Há uma visível possibilidade do surgimento de termos bem diferentes dos usuais $\boldsymbol{P} \cdot \boldsymbol{A}$ e $A^{2}$, e isso é uma forte motivação para investigarmos a fundo todos estes termos até segunda ordem em $1 / E_{g}$.

Para tornar nosso estudo mais organizado, vamos separar em ordens de $1 / E_{g}$ a hamiltoniana de interação, i.e.,

$$
\mathcal{H}_{a}=\mathcal{H}_{a}^{0}+\mathcal{H}_{a}^{1}+\mathcal{H}_{a}^{2}+\mathcal{O}\left(\frac{1}{E_{g}^{3}}\right),
$$

em que $\mathcal{H}_{a}^{j}$ contém exclusivamente as correções apenas da ordem de $1 / E_{g}^{j}$. Apresentarei em seções distintas cada uma das contribuições que estas diferentes ordens fornecem. Inquestionavelmente,

$$
\mathcal{H}_{a}^{0}=\frac{\hbar e}{m c} \boldsymbol{k} \cdot \boldsymbol{A}+\frac{e^{2}}{2 m c^{2}} A^{2}
$$

é a contribuição em ordem zero de $1 / E_{g}$. Ou seja, a contribuição de ordem zero é o único acoplamento que surge na própria banda de condução.

\subsubsection{Ação dos operadores com campo de radiação}

Na seção 2.5.1, estudamos a ação de operadores como $\boldsymbol{T} \cdot \boldsymbol{k} g \boldsymbol{T}^{\dagger} \cdot \boldsymbol{k}$. Desta vez o interesse maior recai sobre operadores como

$$
\boldsymbol{T} \cdot\left(\boldsymbol{k}+\frac{e}{\hbar c} \boldsymbol{A}\right) g \boldsymbol{T}^{\dagger} \cdot\left(\boldsymbol{k}+\frac{e}{\hbar c} \boldsymbol{A}\right)
$$

utilizando a regra 2.5 .5 , a saber

$$
\boldsymbol{T} \cdot \boldsymbol{B} \boldsymbol{T}^{\dagger} \cdot \boldsymbol{C}=\mathbb{1}_{2 \times 2} \boldsymbol{B} \cdot \boldsymbol{C}-\frac{i}{2} \boldsymbol{\sigma} \cdot \boldsymbol{B} \times \boldsymbol{C} .
$$

Expandindo os produtos,

$$
\begin{aligned}
\boldsymbol{T} \cdot\left(\boldsymbol{k}+\frac{e}{\hbar c} \boldsymbol{A}\right) & g \boldsymbol{T}^{\dagger} \cdot\left(\boldsymbol{k}+\frac{e}{\hbar c} \boldsymbol{A}\right)= \\
& \boldsymbol{T} \cdot \boldsymbol{k} g \boldsymbol{T}^{\dagger} \cdot \boldsymbol{k}+\frac{e}{\hbar c} \boldsymbol{T} \cdot \boldsymbol{k} g \boldsymbol{T}^{\dagger} \cdot \boldsymbol{A}+g \frac{e}{\hbar c} \boldsymbol{T} \cdot \boldsymbol{A} \boldsymbol{T}^{\dagger} \cdot \boldsymbol{k}+\left(\frac{e}{\hbar c}\right)^{2} A^{2} g
\end{aligned}
$$


Posso ainda simplificar o termo $\boldsymbol{T} \cdot \boldsymbol{k} g \boldsymbol{T}^{\dagger} \cdot \boldsymbol{A}$ usando a regra do produto,

$$
\boldsymbol{T} \cdot \boldsymbol{k} g \boldsymbol{T}^{\dagger} \cdot \boldsymbol{A}=\boldsymbol{T} \cdot(\boldsymbol{k} g) \boldsymbol{T}^{\dagger} \cdot \boldsymbol{A}+g \boldsymbol{T} \cdot \boldsymbol{k} \boldsymbol{T}^{\dagger} \cdot \boldsymbol{A},
$$

Trivialmente, da identidade 2.5 .5 vale

$$
\boldsymbol{T} \cdot \boldsymbol{k} \boldsymbol{T}^{\dagger} \cdot \boldsymbol{A}=\boldsymbol{A} \cdot \boldsymbol{k}-\frac{i}{2} \boldsymbol{\sigma} \cdot \boldsymbol{k} \times \boldsymbol{A}
$$

Vou definir um campo magnético,

$$
\mathcal{B}:=i \boldsymbol{k} \times \boldsymbol{A}
$$

que mostrou-se na identidade 4.5.14. Ainda com 2.5.5, posso simplificar o outro termo:

$$
\boldsymbol{T} \cdot(\boldsymbol{k} g) \boldsymbol{T}^{\dagger} \cdot \boldsymbol{A}=(\boldsymbol{k} g) \cdot \boldsymbol{A}-\frac{i}{2} \boldsymbol{\sigma} \cdot(\boldsymbol{k} g) \times \boldsymbol{A} .
$$

As equações 4.5.16 e 4.5.14 simplificam 4.5.12 e nos levam ao resultado

$$
\begin{aligned}
& \boldsymbol{T} \cdot\left(\boldsymbol{k}+\frac{e}{\hbar c} \boldsymbol{A}\right) g \boldsymbol{T}^{\dagger} \cdot\left(\boldsymbol{k}+\frac{e}{\hbar c} \boldsymbol{A}\right)= \\
& \boldsymbol{T} \cdot \boldsymbol{k} g \boldsymbol{T}^{\dagger} \cdot \boldsymbol{k}+\frac{e}{\hbar c} \boldsymbol{T} \cdot(\boldsymbol{k} g) \boldsymbol{T}^{\dagger} \cdot \boldsymbol{A}+\left(\frac{e}{\hbar c}\right)^{2} A^{2} g+g \frac{2 e}{\hbar c} \boldsymbol{A} \cdot \boldsymbol{k}-\frac{i e}{2 \hbar c} g \boldsymbol{\sigma} \cdot \boldsymbol{k} \times \boldsymbol{A} .
\end{aligned}
$$

O primeiro termo desta equação pode ser simplificado usando as mesmas técnicas que apresentei ao longo do capítulo2. A equação 4.5.17 é uma extensão da identidade 2.5.13, deduzida no capítulo 2 sem campo eletromagnético. Na equação 4.5.17 há um termo com um campo magnético 4.5.15. Vou mantê-lo e mais adiante o analisarei com cuidado, mas não posso deixar de alertar que $\boldsymbol{\sigma} \cdot \mathcal{B}$ assemelha-se ao termo Zeeman.

\subsubsection{Termos quadráticos no potencial vetor}

Os termos quadráticos em $\boldsymbol{A}$ na equação 4.5 .9 , i.e.,

$$
\left(\frac{e}{\hbar c}\right)^{2}\left\{\boldsymbol{T} \cdot \boldsymbol{A} \frac{2}{\epsilon-E_{8}} \boldsymbol{T}^{\dagger} \cdot \boldsymbol{A}+\boldsymbol{\sigma} \cdot \boldsymbol{A} \frac{1}{\epsilon-E_{7}} \boldsymbol{\sigma} \cdot \boldsymbol{A}\right\},
$$

podem ser simplificados sem quaisquer aproximações, uma vez que não há operadores diferenciais. Usando a regra 2.5.5, vale

$$
\boldsymbol{T} \cdot \boldsymbol{A} \frac{2}{\epsilon-E_{8}} \boldsymbol{T}^{\dagger} \cdot \boldsymbol{A}=\frac{2}{\epsilon-E_{8}} A^{2},
$$

uma vez que $\boldsymbol{A} \times \boldsymbol{A}=0$. Similarmente,

$$
\boldsymbol{\sigma} \cdot \boldsymbol{A} \frac{1}{\epsilon-E_{7}} \boldsymbol{\sigma} \cdot \boldsymbol{A}=\frac{1}{\epsilon-E_{7}} A^{2} .
$$


Assim, o acoplamento com as bandas de valência gera, de forma exata (em todas as ordens de $\left.1 / E_{g}\right)$, o seguinte acoplamento quadrático em $\boldsymbol{A}$ entre os elétrons de condução e fótons

$$
\mathcal{H}_{a}^{A^{2}}=\left(\frac{e}{\hbar c}\right)^{2}\left(\frac{2}{\epsilon-E_{8}}+\frac{1}{\epsilon-E_{7}}\right) A^{2} .
$$

Os próximos cálculos evolvem aproximações nestes denominadores de energia e, portanto, não faz sentido mantermos $\mathcal{H}_{a}^{A^{2}}$ desta forma. Aproximando em segunda ordem de $1 / E_{g}$,

$$
\mathcal{H}_{a}^{A^{2}}=\left(\frac{e}{\hbar c}\right)^{2}\left(\gamma_{1}+\gamma_{2} V+\gamma_{2}^{\prime} h_{w}+\gamma_{2} \epsilon\right) A^{2}
$$

Esta dependência na energia desaparecerá quando acertarmos a normalização, tal como ocorre com a hamiltoniana efetiva da banda de condução sem campo de radiação.

\subsubsection{Normalização}

Trocarei o spinor $\psi$ por outro $\psi_{S}$, também de duas componentes, mas que leve em conta as bandas de valência em sua normalização. Desta forma, não precisaremos mais das bandas de valência e obteremos com um modelo efetivo mesmo na presença de campos eletromagnéticos. Até segunda ordem em $1 / E_{g}$, temos, das equações $4.5 .3 \mathrm{a}$ e $4.5 .3 \mathrm{~b}$,

$$
\begin{gathered}
\phi=P \sqrt{\frac{2}{3}}\left(1-\frac{\epsilon-V+h_{8}}{E_{g}}+\frac{\hbar^{2}}{2 m E_{g}}\left(\boldsymbol{k}+\frac{e}{\hbar c} \boldsymbol{A}\right)^{2}\right) \boldsymbol{T}^{\dagger} \cdot\left(\boldsymbol{k}+\frac{e}{\hbar c} \boldsymbol{A}\right) \psi \quad \mathrm{e} \\
\chi=\frac{P}{\sqrt{3}}\left(1-\frac{\epsilon-V+h_{7}}{E_{g}+\Delta_{g}}+\frac{\hbar^{2}}{2 m\left(E_{g}+\Delta_{g}\right)}\left(\boldsymbol{k}+\frac{e}{\hbar c} \boldsymbol{A}\right)^{2}\right) \boldsymbol{\sigma} \cdot\left(\boldsymbol{k}+\frac{e}{\hbar c} \boldsymbol{A}\right) \psi
\end{gathered}
$$

Assim, a densidade de probabilidade torna-se

$$
\begin{aligned}
|\Psi|^{2}=|\psi|^{2}+\frac{2 P^{2}}{3 E_{g}^{2}}\left|\boldsymbol{T}^{\dagger} \cdot\left(\boldsymbol{k}+\frac{e}{\hbar c} \boldsymbol{A}\right) \psi\right|^{2}+ & \\
& \frac{P^{2}}{3\left(E_{g}+\Delta_{g}\right)^{2}}\left|\boldsymbol{\sigma} \cdot\left(\boldsymbol{k}+\frac{e}{\hbar c} \boldsymbol{A}\right) \psi\right|^{2}+\mathcal{O}\left(\frac{1}{E_{g}^{4}}\right) .
\end{aligned}
$$

A menos da substituição mínima, a equação 4.5 .23 é idêntica à 2.7.13, que é a densidade de probabilidade na ausência de campo. Portanto, seguirei os mesmos argumentos usados na seção 2.7.1 para derivar este novo spinor $\psi_{S}$. Tomando o resultado da seção e usando a substituição mínima, obtemos

$$
\psi=\left[1-\frac{P^{2}}{6} \gamma_{2}\left(\boldsymbol{k}+\frac{e}{\hbar c} \boldsymbol{A}\right)^{2}\right] \psi_{S}+\mathcal{O}\left(\frac{1}{E_{g}^{2}}\right) .
$$

Os cálculos são todos exatamente iguais, e não há grandes dificuldades para mostrar essa identidade. 


\subsection{Interação elétrons-fóton em primeira ordem}

Aplicarei as aproximações nos denominadores de energia na hamiltoniana de interação da equação 4.5 .9 até primeira ordem em $1 / E_{g}$. Usando as aproximações 2.6 .1 e 2.6.2, obtive

$$
\begin{aligned}
\mathcal{H}_{a}^{1}= & \frac{e}{\hbar c}\left\{\frac{2}{E_{g}} \boldsymbol{T} \cdot \boldsymbol{A} \boldsymbol{T}^{\dagger} \cdot \boldsymbol{k}+\frac{2}{E_{g}} \boldsymbol{T} \cdot \boldsymbol{k} \boldsymbol{T}^{\dagger} \cdot \boldsymbol{A}+\frac{1}{E_{g}+\Delta_{g}} \boldsymbol{\sigma} \cdot \boldsymbol{A} \boldsymbol{\sigma} \cdot \boldsymbol{k}+\right. \\
& \left.\frac{1}{E_{g}+\Delta_{g}} \boldsymbol{\sigma} \cdot \boldsymbol{k} \boldsymbol{\sigma} \cdot \boldsymbol{A}\right\}+\left(\frac{e}{\hbar c}\right)^{2}\left\{\frac{2}{E_{g}} \boldsymbol{T} \cdot \boldsymbol{A} \boldsymbol{T}^{\dagger} \cdot \boldsymbol{A}+\frac{1}{E_{g}+\Delta_{g}} \boldsymbol{\sigma} \cdot \boldsymbol{A} \boldsymbol{\sigma} \cdot \boldsymbol{A}\right\} .
\end{aligned}
$$

Até primeira ordem em $1 / E_{g}$, a equação 2.7 .13 garante que $\psi=\psi_{S}$ e, portanto, não há necessidade de corrigir a normalização.

Separadamente, tratarei os termos que envolvem as matrizes $\boldsymbol{T}$ e $\boldsymbol{\sigma}$. Com a regra 2.5.5, é fácil de mostrar que

$$
\boldsymbol{T} \cdot \boldsymbol{A} \boldsymbol{T}^{\dagger} \cdot \boldsymbol{k}+\boldsymbol{T} \cdot \boldsymbol{k} \boldsymbol{T}^{\dagger} \cdot \boldsymbol{A}=\boldsymbol{A} \cdot \boldsymbol{k}-\frac{i}{2} \boldsymbol{\sigma} \cdot \boldsymbol{A} \times \boldsymbol{k}+\boldsymbol{k} \cdot \boldsymbol{A}-\frac{i}{2} \boldsymbol{\sigma} \cdot \boldsymbol{k} \times \boldsymbol{A} .
$$

Como $\nabla \cdot \boldsymbol{A}=0^{* *}$, então

$$
\boldsymbol{k} \cdot \boldsymbol{A}=k_{x} A_{x}+k_{y} A_{y}+k_{z} A_{z}=A_{x} k_{x}+A_{y} k_{y}+A_{z} k_{z}=\boldsymbol{A} \cdot \boldsymbol{k} .
$$

No entanto,

$$
\boldsymbol{k} \times \boldsymbol{A}=-\boldsymbol{A} \times \boldsymbol{k} .
$$

Usando as identidades 4.6 .3 e 4.6.4 na equação 4.6.2, chegamos por fim em

$$
\frac{2}{E_{g}}\left(\boldsymbol{T} \cdot \boldsymbol{A} \boldsymbol{T}^{\dagger} \cdot \boldsymbol{k}+\boldsymbol{T} \cdot \boldsymbol{k} \boldsymbol{T}^{\dagger} \cdot \boldsymbol{A}\right)=\frac{4}{E_{g}} \boldsymbol{A} \cdot \boldsymbol{k} .
$$

Voltando à equação 4.6.1, os cálculos com os termos que envolvem as matrizes de Pauli $\boldsymbol{\sigma}$ são similares, e como resultado temos

$$
\frac{2}{E_{g}+\Delta_{g}}(\boldsymbol{\sigma} \cdot \boldsymbol{A} \boldsymbol{\sigma} \cdot \boldsymbol{k}+\boldsymbol{\sigma} \cdot \boldsymbol{k} \boldsymbol{\sigma} \cdot \boldsymbol{A})=\frac{2}{E_{g}+\Delta_{g}} \boldsymbol{A} \cdot \boldsymbol{k}
$$

Reunindo, por fim, os termos 4.6.5 e 4.6.6, adicionando o termo de primeira ordem da equação 4.5.21,

$$
\mathcal{H}_{a}^{0}+\mathcal{H}_{a}^{1}=\frac{2 e}{\hbar c}\left(\frac{\hbar^{2}}{2 m}+\frac{\mathcal{P}^{2}}{3} \gamma_{1}\right) \boldsymbol{k} \cdot \boldsymbol{A}+\frac{e^{2}}{\hbar^{2} c^{2}}\left(\frac{\hbar^{2}}{2 m}+\frac{\mathcal{P}^{2}}{3} \gamma_{1}\right) A^{2}-\frac{\mathcal{P}^{2}}{3} \gamma_{1} \boldsymbol{\sigma} \cdot \mathcal{B}
$$

Estes acoplamentos apresentam os usuais $\boldsymbol{P} \cdot \boldsymbol{A}$ e $A^{2}$ com uma correção de massa efetiva de primeira ordem em $1 / E_{g}$ (compare com a equação 2.7.28). Além disso, vemos uma correção

**É importante deixar claro que isso não significa que $\left[k_{j}, A_{p}\right]=0$. 
Zeeman efetiva. Da mesma forma como decorre da equação de Dirac, na presença de campos eletromagnéticos, o termo Zeeman, obtemos aqui um termo Zeeman efetivo para a banda de condução. Podemos definir um fator giromagnético efetivo,

$$
g_{1}:=\frac{\mathcal{P}^{2}}{3 \mu_{B}} \gamma_{1},
$$

em que $\mu_{B}$ é o magneton de Bohr. Desta forma, a interação Zeeman efetiva que obtemos pode ser escrita como $-\mu_{B} g_{1} \sigma \cdot \mathcal{B}$. O subescrito 1 denota primeira ordem em $1 / E_{g}$. Mostrarei na seção 4.7 que, ao contrário do que ocorre com a aproximação relativística de segunda ordem em $1 / c$ na equação de Dirac, obteremos uma "segunda correção Zeeman" da ordem de $1 / E_{g}^{2}$.

\subsection{Interação elétrons-fóton até segunda ordem}

Vamos mais fundo: mostrarei quais contribuições ocorrem em segunda ordem de $1 / E_{g}$. Desta vez teremos que tratar de operadores diferenciais agindo em funções como o potencial de interação $(V)$ e os potenciais estruturais $\left(h_{6}, h_{7}\right.$ e $\left.h_{8}\right)$. Poderemos ver o surgimento de operadores mais interessantes - e que possam depender de spin. Os termos exclusivamente de segunda ordem em $1 / E_{g}$ são

$$
\begin{gathered}
\mathcal{H}_{a}^{2}=\frac{2 e}{\hbar c E_{g}^{2}}\left\{\boldsymbol{T} \cdot \boldsymbol{A}\left(V-\epsilon-h_{8}+\frac{\hbar^{2} \tilde{k}^{2}}{2 m}\right) \boldsymbol{T}^{\dagger} \cdot \boldsymbol{k}+\boldsymbol{T} \cdot \boldsymbol{k}\left(V-\epsilon-h_{8}+\frac{\hbar^{2} \tilde{k}^{2}}{2 m}\right) \boldsymbol{T}^{\dagger} \cdot \boldsymbol{A}\right\}+ \\
\frac{e}{\hbar c\left(E_{g}+\Delta_{g}\right)^{2}}\left\{\boldsymbol{\sigma} \cdot \boldsymbol{A}\left(V-\epsilon-h_{7}+\frac{\hbar^{2} \tilde{k}^{2}}{2 m}\right) \boldsymbol{\sigma} \cdot \boldsymbol{k}+\boldsymbol{\sigma} \cdot \boldsymbol{k}\left(V-\epsilon-h_{7}+\frac{\hbar^{2} \tilde{k}^{2}}{2 m}\right) \boldsymbol{\sigma} \cdot \boldsymbol{A}\right\}+ \\
\left(\frac{e}{\hbar c}\right)^{2}\left(V-\epsilon-h_{8}+\frac{\hbar^{2} \tilde{k}^{2}}{2 m}\right)\left\{\frac{2}{E_{g}^{2}} \boldsymbol{T} \cdot \boldsymbol{A} \boldsymbol{T}^{\dagger} \cdot \boldsymbol{A}+\frac{1}{\left(E_{g}+\Delta_{g}\right)^{2}} \boldsymbol{\sigma} \cdot \boldsymbol{A} \boldsymbol{\sigma} \cdot \boldsymbol{A}\right\}, \quad(4.7 .1)
\end{gathered}
$$

em que defini implicitamente

$$
\tilde{\boldsymbol{k}}:=\boldsymbol{k}+\frac{e}{\hbar c} \boldsymbol{A}
$$

para poupar algum espaço e facilitar a leitura de 4.7.1. Apesar de serem muitos termos, são todas contas muito similares às da seção 2.7. A dependência quadrática em $\boldsymbol{A}$ pode ser rapidamente simplificada como 4.5.21. Por simplicidade, vamos adicionar este termo apenas no final dos cálculos. 
Vou desenvolver primeiramente os produtos com o termo cinético, i.e.,

$$
\begin{array}{r}
\boldsymbol{T} \cdot \boldsymbol{A} \tilde{k}^{2} \boldsymbol{T}^{\dagger} \cdot \boldsymbol{k}=\boldsymbol{T} \cdot \boldsymbol{A}\left(\boldsymbol{k}+\frac{2 e}{\hbar c} \boldsymbol{A}\right)^{2} \boldsymbol{T}^{\dagger} \cdot \boldsymbol{k}=\boldsymbol{T} \cdot \boldsymbol{A}\left(k^{2}+\frac{e}{\hbar c} \boldsymbol{k} \cdot \boldsymbol{A}+\frac{e}{\hbar c} A^{2}\right) \boldsymbol{T}^{\dagger} \cdot \boldsymbol{k} \\
=\left(k^{2}+\frac{2 e}{\hbar c} \boldsymbol{k} \cdot \boldsymbol{A}+\frac{e}{\hbar c} A^{2}\right)\left\{\boldsymbol{A} \cdot \boldsymbol{k}-\frac{i}{2} \boldsymbol{\sigma} \cdot \boldsymbol{A} \times \boldsymbol{k}\right\}
\end{array}
$$

Similarmente, vale

$$
\boldsymbol{\sigma} \cdot \boldsymbol{A} \tilde{k}^{2} \boldsymbol{\sigma} \cdot \boldsymbol{k}=\left(k^{2}+\frac{2 e}{\hbar c} \boldsymbol{k} \cdot \boldsymbol{A}+\frac{e}{\hbar c} A^{2}\right)\{\boldsymbol{A} \cdot \boldsymbol{k}+i \boldsymbol{\sigma} \cdot \boldsymbol{A} \times \boldsymbol{k}\}
$$

Além destes dois termos, vemos na equação 4.7 .1 outros dois em que $\boldsymbol{A}$ e $\boldsymbol{k}$ trocam de posições. Desenvolvendo-os de forma similar, obtemos resultados similares aos das equações 4.7.3 e 4.7.4, mas com sinais opostos nos termos com spin. Isso significa que os produtos vetoriais são cancelados. Reunindo todos os termos e utilizando as condições 4.6 .3 e 4.6 .4 novamente, obtive

$$
\begin{aligned}
\frac{2 e}{\hbar c} \frac{\hbar^{2}}{2 m}\left\{\boldsymbol{T} \cdot \boldsymbol{A} \frac{\tilde{k}^{2}}{E_{g}^{2}} \boldsymbol{T}^{\dagger} \cdot \boldsymbol{k}+\boldsymbol{T} \cdot \boldsymbol{k} \frac{\tilde{k}^{2}}{E_{g}^{2}} \boldsymbol{T}^{\dagger} \cdot \boldsymbol{A}+\boldsymbol{\sigma} \cdot \boldsymbol{A} \frac{\tilde{k}^{2}}{\left(E_{g}+\Delta_{g}\right)^{2}} \boldsymbol{\sigma} \cdot \boldsymbol{k}+\right. \\
\left.\boldsymbol{\sigma} \cdot \boldsymbol{k} \frac{\tilde{k}^{2}}{\left(E_{g}+\Delta_{g}\right)^{2}} \boldsymbol{\sigma} \cdot \boldsymbol{A}\right\}=\frac{2 \hbar e}{m c} \gamma_{2} \tilde{k}^{2} \boldsymbol{A} \cdot \boldsymbol{k} .
\end{aligned}
$$

A constante $\gamma_{2}^{\prime}$ foi definida na equação 2.7.10. A priori, este termo representa mais uma contribuição para o acoplamento do tipo $\boldsymbol{P} \cdot \boldsymbol{A}$. Mais tarde voltarei à equação 4.7 .5 e veremos que esta contribuição também será cancelada via normalização.

Tratarei agora os termos que envolvem a energia $\epsilon$, que é apenas um número. Simplificando todos os termos, sobra

$$
-\epsilon \gamma_{2} \boldsymbol{A} \cdot \boldsymbol{k}
$$

Esta contribuição desaparecerá quando eu adicionar a renormalização e voltar a ter operadores independentes da energia.

Simplificarei a seguir os termos da equação 4.7 .1 que começam com $\boldsymbol{T} \cdot \boldsymbol{A}$ ou $\boldsymbol{\sigma} \cdot \boldsymbol{A}$. Por $\boldsymbol{A}$ não ser um operador diferencial, simplificar estes termos é uma tarefa mais simples. $\mathrm{O}$ primeiro termo pode ser simplificado sem mistério algum usando a regra 2.5.5,

$$
\boldsymbol{T} \cdot \boldsymbol{A}\left(V-h_{8}\right) \boldsymbol{T}^{\dagger} \cdot \boldsymbol{k}=\left(V-h_{8}\right)\left\{\boldsymbol{A} \cdot \boldsymbol{k}-\frac{i}{2} \boldsymbol{\sigma} \cdot \boldsymbol{A} \times \boldsymbol{k}\right\} .
$$

Similarmente, o terceiro termo da equação 4.7.1 fica

$$
\boldsymbol{\sigma} \cdot \boldsymbol{A}\left(V-h_{7}\right) \boldsymbol{\sigma} \cdot \boldsymbol{k}=\left(V-h_{7}\right)\{\boldsymbol{A} \cdot \boldsymbol{k}+i \boldsymbol{\sigma} \cdot \boldsymbol{A} \times \boldsymbol{k}\} .
$$


Revisitaremos estas duas últimas igualdades para reunirmos os termos. De imediato, note algo no mínimo curioso: o surgimento de operadores não hermitianos. É fácil verificar que os termos com $\sigma$, do lado direito das equações 4.7 .7 e 4.7.8, não são hermitianos ${ }^{\dagger \dagger}$. Cada ordem de uma expansão em Taylor de um operador hermitiano é, necessariamente, hermitiana (veja o apêndice A.3 para uma breve discussão sobre isso).

Os termos restantes na equação 4.7.1 envolvem o desenvolvimento de expressões como na equação 4.5.16, em que o papel de $g$ é desempenhado pelos potenciais $V, h_{7}$ e $h_{8}$. O desenvolvimento com as matrizes de pauli é bem similar. Como resultado destes cálculos, temos

$$
\begin{array}{r}
\frac{2}{E_{g}^{2}} \boldsymbol{T} \cdot \boldsymbol{k}\left(V-h_{8}\right) \boldsymbol{T}^{\dagger} \cdot \boldsymbol{A}+\frac{1}{\left(E_{g}+\Delta_{g}\right)^{2}} \boldsymbol{\sigma} \cdot \boldsymbol{k}\left(V-h_{7}\right) \boldsymbol{\sigma} \cdot \boldsymbol{A}= \\
\left(\gamma_{2} V+\gamma_{2}^{\prime} h_{w}\right)\{\boldsymbol{k} \cdot \boldsymbol{A}+i \boldsymbol{\sigma} \cdot \boldsymbol{k} \times \boldsymbol{A}\}-\left(\eta_{H} V+\gamma_{2}^{\prime} h_{w}\right) \boldsymbol{\sigma} \cdot \mathcal{B}+ \\
\gamma_{2} \boldsymbol{k} V \cdot \boldsymbol{A}+\gamma_{2}^{\prime} \boldsymbol{k} h_{w} \cdot \boldsymbol{A}-i \boldsymbol{\sigma} \cdot\left(\boldsymbol{k} h_{w}+\boldsymbol{k} V\right) \times \boldsymbol{A} .
\end{array}
$$

Somando 4.7 .9 com 4.7.7 e 4.7.8 e relembrando a equação 4.6.4, observe o cancelamento de quase todos os operadores não hermitianos. Note o surgimento, já anunciado na seção passada, de uma correção Zeeman de segunda ordem.

Reunindo todos os termos que coletamos até o momento - listados nas equações 4.7.54.7.9, temos

$$
\begin{array}{r}
\mathcal{H}_{a}^{2}=\gamma_{2} V \boldsymbol{k} \cdot \boldsymbol{A}+\gamma_{2}^{\prime} h_{w} \boldsymbol{k} \cdot \boldsymbol{A}+\gamma_{2} \boldsymbol{k} V \cdot \boldsymbol{A}+\gamma_{2}^{\prime} \boldsymbol{k} h_{w} \cdot \boldsymbol{A}-i \boldsymbol{\sigma} \cdot\left(\boldsymbol{k} h_{w}+\boldsymbol{k} V\right) \times \boldsymbol{A}+ \\
\frac{2 \hbar e}{m c} \gamma_{2} \boldsymbol{A} \cdot \boldsymbol{k}-\epsilon \gamma_{2}\{\boldsymbol{A} \cdot \boldsymbol{k}+i \boldsymbol{\sigma} \cdot \boldsymbol{A} \times \boldsymbol{k}\}+\left(\gamma_{2} V+\gamma_{2}^{\prime} h_{w}+\gamma_{2} \epsilon\right) A^{2} .
\end{array}
$$

É importante ressaltar que temos operadores que dependem de spin. Portanto, esta interação elétron-fóton pode causar uma mistura nos estados de spin, o que significa indução de transição entre estados com spin $|+\rangle$ e $|-\rangle$.

Antes de continuarmos, corrigirei a normalização do spinor da banda de condução. E esta correção deve ser realizada usando o mesmo procedimento que usamos na seção 2.7.2: multiplicando, pela esquerda e pela direita, a hamiltoniana efetiva pelo operador

$$
\mathcal{L}:=\left[1-\frac{P^{2}}{6} \gamma_{2}\left(\boldsymbol{k}+\frac{e}{\hbar c} \boldsymbol{A}\right)^{2}\right] .
$$

Isso garante a consistência do que estamos calculando agora com o que deduzimos no capítulo

${ }^{\dagger \dagger}$ Verifique primeiro que $\boldsymbol{A}, \boldsymbol{k}$ e $\boldsymbol{\sigma}$ são hermitianos. Depois verifique se o operador $i \boldsymbol{\sigma} \cdot \boldsymbol{A} \times \boldsymbol{k}$ é hermitiano. A primeira verificação nega a segunda (ou vice-versa). Logo mais estes termos serão todos cancelados. 
2. Neste caso, $\mathcal{H}_{0}$, a parte de $\mathcal{H}$ que independe do potencial vetor, se tornará exatamente a hamiltoniana dada por 2.7.27, cujos estados e energias são conhecidas. Temos então

$$
\left(\mathcal{H}_{0}+\frac{\mathcal{P}^{2}}{3}\left[\mathcal{H}_{a}^{0}+\mathcal{H}_{a}^{1}+\mathcal{H}_{a}^{2}\right]\right) \psi=\left(\epsilon-V-h_{6}-\frac{\hbar^{2} k^{2}}{2 m}\right) \psi .
$$

Vale lembrar que $\mathcal{H}_{a}^{0}$ é a parte de $E_{6}$ que depende do potencial vetor, e por isso na equação 4.7.12 colocamos explicitamente a parte cinética dependendo apenas de $k^{2}$. Trocando $\psi$ por $\psi_{S}$ e multiplicando pela esquerda por $\mathcal{L}$, temos

$$
\mathcal{L}\left(\mathcal{H}_{0}+\mathcal{H}_{a}^{0}+\mathcal{H}_{a}^{1}+\mathcal{H}_{a}^{2}\right) \mathcal{L} \psi_{S}=\mathcal{L}\left(\epsilon-V-h_{6}-\frac{\hbar^{2} k^{2}}{2 m}\right) \mathcal{L} \psi_{S} .
$$

É fácil ver que os termos de segunda ordem ficam inalterados, pois $\mathcal{L}$ também é de segunda ordem (o que gera, no mínimo, termos de terceira ordem) ${ }^{\ddagger \ddagger}$. Desta forma,

$$
\mathcal{L}\left(\mathcal{H}_{a}^{0}+\mathcal{H}_{a}^{1}+\mathcal{H}_{a}^{2}\right) \mathcal{L}=\mathcal{L} \mathcal{H}_{a}^{0} \mathcal{L}+\mathcal{H}_{a}^{1}+\mathcal{H}_{a}^{2}+\mathcal{O}\left(\frac{1}{E_{g}^{3}}\right)
$$

Portanto, os produtos do lado esquerdo de 4.7 .13 são bem simples, e até segunda ordem em $1 / E_{g}$ ficamos com

$$
\mathcal{L} \mathcal{H}_{a}^{0} \mathcal{L}=\frac{2 e}{\hbar c}\left(\frac{\hbar^{2}}{2 m}-\frac{\mathcal{P}^{2} \hbar^{2}}{6 m} \gamma_{2} k^{2}\right) \boldsymbol{k} \cdot \boldsymbol{A}+\frac{e^{2}}{\hbar^{2} c^{2}}\left(\frac{\hbar^{2}}{6 m}-\frac{\mathcal{P}^{2} \hbar^{2}}{6 m} \gamma_{2} k^{2}\right) A^{2} .
$$

No lado direito de 4.7.13, temos

$$
\begin{aligned}
\mathcal{L}(\epsilon-V & \left.-h_{6}-\frac{\hbar^{2} k^{2}}{2 m}\right) \mathcal{L}=\epsilon-V-h_{6}-\frac{\hbar^{2} k^{2}}{2 m}-\epsilon \frac{\mathcal{P}^{2}}{3} \gamma_{2}\left(k^{2}+\frac{e^{2}}{\hbar^{2} c^{2}} A^{2}+\frac{e}{\hbar c} \boldsymbol{k} \cdot \boldsymbol{A}\right) \\
& +\frac{\mathcal{P}^{2} e}{3 \hbar c} \gamma_{2} \frac{\left(V+h_{6}\right) \boldsymbol{k} \cdot \boldsymbol{A}+2 \boldsymbol{k}\left(V+h_{6}\right) \cdot \boldsymbol{A}}{2}+2 \frac{\mathcal{P}^{2} e^{2}}{3 \hbar^{2} c^{2}} A^{2} \frac{V+h_{6}}{2} \\
+ & \frac{\mathcal{P}^{2}}{3} \gamma_{2} \frac{\left(k^{2} V+k^{2} h_{6}\right)+2\left(V+h_{6}\right) k^{2}+2 \boldsymbol{k}\left(V+h_{6}\right) \cdot \boldsymbol{k}+\left(V+h_{6}\right) k^{2}}{2}
\end{aligned}
$$

Os termos independentes do potencial vetor não interferem nestes cálculos (veja a terceira linha): a união de todos os termos independentes do potencial vetor reconstroem a hamiltoniana 2.7.27, que passarei a chamar de $\mathcal{H}_{0}$ (reveja a equação 4.5.5). Esta é a parte puramente eletrônica da hamiltoniana $\mathcal{H}$.

Comparando 4.7.16 e 4.5.21 fica evidente que todos os termos com energia se cancelam. Finalizando, basta substituir 4.7.16 e 4.7.15 na equação 4.7.13 para obter uma equação efetiva para a banda de condução na presença de campos eletromagnéticos:

$$
\left[H_{\mathrm{eff}}+W_{\mathrm{eff}}-\mu_{B}\left(g_{1}+g_{2}\right) \boldsymbol{\sigma} \cdot \mathcal{B}\right] \psi_{S}=\left(\epsilon-V-h_{6}\right) \psi_{S}
$$

$\ddagger \ddagger$ Ocorre o mesmo na equação 2.7 .24 . 
em que $H_{\text {eff }}$ é exatamente igual à 2.7 .27 e

$$
W_{\mathrm{eff}}=\frac{\hbar}{2 M} \boldsymbol{k} \cdot \boldsymbol{A}+\frac{\alpha_{M} e}{\hbar c} \boldsymbol{k} h_{w} \cdot \boldsymbol{A}-\frac{i e}{\hbar c} \boldsymbol{\sigma} \cdot \boldsymbol{k} \tilde{\eta} \times \boldsymbol{A}+\frac{e^{2}}{2 M c^{2}} A^{2}
$$

são as interações elétron de condução - fóton até segunda ordem em $1 / E_{g}$ com normalização já corrigida. Utilizei a mesma notação para as contantes que no capítulo 3, i.e.,

$$
\begin{aligned}
\frac{\hbar^{2}}{2 M} & :=\frac{\hbar^{2}}{2 m}+\frac{2}{E_{g}}+\frac{1}{E_{g}+\Delta_{g}}+\left[\frac{2 \delta_{8}}{E_{g}^{2}}+\frac{\delta_{7}}{\left(E_{g}+\Delta_{g}\right)^{2}}+\gamma_{2} \delta_{6}\right] h_{w} \\
\alpha_{M} & :=-\frac{\mathcal{P}^{2}}{3}\left[\gamma_{2}^{\prime}+\gamma_{2} \delta_{6}\right]
\end{aligned}
$$

Além disso, defini implicitamente a função

$$
\tilde{\eta}:=\gamma_{H} V+\gamma_{e} h_{w}
$$

A função $\eta$ da equação 2.7.38, que determina os acoplamentos spin-órbita $\eta_{i j}$, pode ser escrita como $i \boldsymbol{k} \tilde{\eta}$. Conscientemente, mantive a interação Zeeman separada da interação elétronfóton $^{\S \S}$, e defini

$$
g_{2}=\frac{\mathcal{P}^{2}}{3 \mu_{B}} \tilde{\eta}
$$

como fator giromagnético efetivo da correção de segunda ordem. Esta correção dependente da posição e muda abruptamente com a mudança de material. Note que as correções Zeeman são bem parecidas com a correção da massa efetiva. Apesar de obtermos uma correção Zeeman induzida pelas bandas de valência, é necessário lembrar que na hamiltoniana $\mathbf{k} \cdot \mathbf{p}$ inicial, dada na equação 4.1.7, não incluímos o termo Zeeman. Portanto, pode haver outras contribuições a serem somadas a este efeito Zeeman. Ainda assim, os termos com campo magnético do proceedings de Winkler ${ }^{41}$ concorda por completo com nossa expressão.

\subsection{Interação dependente do tempo}

Um fóton é uma partícula associada às oscilações dos campos eletromagnéticos. Como ambos os campos oscilam, o vetor potencial $\boldsymbol{A}$, já quantizado na equação 4.2 .16 , também oscila no tempo. Um detalhe que deixei até o momento de lado, mas que representa uma grande dificuldade (talvez a maior) na solução deste problema, é a dependência temporal nos acoplamentos 4.7.18. A seguir, mostrarei como tratar esta interação dependente do tempo, utilizando a mesma abordagem no curso de Madelung ${ }^{86}$.

\footnotetext{
$\S \S \mathrm{Na}$ maioria das referências ${ }^{7,84,85}$, estes acoplamentos são tratados de forma separada.
} 
A equação 4.7.17 divide a hamiltoniana em duas contribuições,

$$
\mathcal{H}=H_{\text {eff }}+W_{\text {eff }}
$$

intrinsecamente diferentes: $W_{\text {eff }}$ é a única contribuição dependente do tempo. Sua dependência no tempo decorre exclusivamente do potencial vetor $\boldsymbol{A}$. Conhecemos as soluções (estados estacionários) da porção independente do tempo, i.e., as soluções de

$$
H_{\mathrm{eff}}\left|\Psi_{n \boldsymbol{k}}\right\rangle=\left(E_{n \boldsymbol{k}}-V-h_{6}\right)\left|\Psi_{n \boldsymbol{k}}\right\rangle
$$

em que $n$ etiquetam as diversas soluções. Estas soluções são dadas na seção 3.6 com $n=$ $\left(\lambda_{1}, \lambda_{2}\right)$. Como estes são os estados estacionários, uma solução geral ${ }^{\text {đq }}$ desta hamiltoniana independente do tempo pode ser escrita como

$$
|\Omega(t)\rangle=\sum_{n, \boldsymbol{k}} a_{n \boldsymbol{k}} \exp \left(-\frac{i E_{n \boldsymbol{k}}}{\hbar} t\right)\left|\Psi_{n \boldsymbol{k}}\right\rangle .
$$

Para a parte dependente, podemos aplicar um procedimento bem parecido com o método conhecido com variação de parâmetros. Trata-se de propor como ansatz para a hamiltoniana completa a solução

$$
|\Omega(t)\rangle=\sum_{n, \boldsymbol{k}} a_{n \boldsymbol{k}}(t) \exp \left(-\frac{i E_{n \boldsymbol{k}}}{\hbar} t\right)\left|\Psi_{n \boldsymbol{k}}\right\rangle,
$$

em que uma dependência adicional é atribuída aos coeficientes $a_{n \boldsymbol{k}}$. É fácil de ver que se a parte dependente do tempo for nula (por algum motivo), então a solução torna-se imediatamente 4.8.3. A condição incial torna-se conhecer todos os $a_{n k}(0)$, i.e.,

$$
|\Omega(0)\rangle=\sum_{n, \boldsymbol{k}} a_{n \boldsymbol{k}}(0)\left|\Psi_{n \boldsymbol{k}}\right\rangle
$$

O estado $\left|\Omega_{\boldsymbol{k}}(t)\right\rangle$ fica completamente determinado se descobrirmos cada um dos coeficientes $a_{n \boldsymbol{k}}(t)$. Neste sentido, a equação de Schrödinger dependente do tempo,

$$
i \hbar \frac{d}{d t}|\Omega(t)\rangle=H^{\prime}|\Omega(t)\rangle
$$

pode ser encarada como uma equação de evolução para os coeficientes $a_{n \boldsymbol{k}}(t)$, pois seu lado esquerdo representa

$$
\left\langle\Psi_{j \boldsymbol{k}^{\prime}}\left|\exp \left(\frac{i E_{j \boldsymbol{k}^{\prime}}}{\hbar} t\right) i \hbar \frac{d}{d t}\right| \Omega(t)\right\rangle=i \hbar \frac{d a_{j \boldsymbol{k}^{\prime}}}{d t}(t)+a_{j \boldsymbol{k}^{\prime}}(t) E_{j \boldsymbol{k}^{\prime}}
$$

T⿻ Por solução geral entenda a solução da equação de Schrödinger independente do tempo. Os estados estacionários são soluções em que a hamiltoniana é separável no tempo. 
Igualando com o lado direito obtemos uma equação diferencial ordinária para os coeficientes $a_{n \boldsymbol{k}}(t)$ com condições iniciais já estabelecidas na equação 4.8.5. Multliplicando por $\left\langle\Psi_{j \boldsymbol{k}^{\prime}}\right| \exp \left(\frac{i E_{j \boldsymbol{k}^{\prime}}}{\hbar} t\right)$ o lado direito da equação 4.8.6, obtemos

$$
\left\langle\Psi_{j \boldsymbol{k}^{\prime}}\left|\exp \left(\frac{i E_{j \boldsymbol{k}^{\prime}}}{\hbar} t\right) H^{\prime}\right| \Omega_{\boldsymbol{k}}(t)\right\rangle=\sum_{n, \boldsymbol{k}} a_{n \boldsymbol{k}}(t) e^{i \frac{E_{j \boldsymbol{k}^{\prime}}-E_{n \boldsymbol{k}}}{\hbar} t}\left\langle\Psi_{j \boldsymbol{k}^{\prime}}\left|H^{\prime}\right| \Psi_{n \boldsymbol{k}}\right\rangle .
$$

Por simplicidade e pela frequência com que usaremos a definição a seguir,

$$
\omega_{j \boldsymbol{k}^{\prime} ; n \boldsymbol{k}}:=\frac{E_{j \boldsymbol{k}^{\prime}}-E_{n \boldsymbol{k}}}{\hbar} .
$$

Substituindo $H^{\prime}=H_{k p}+W_{k p}(\boldsymbol{k})+H_{e f}(\boldsymbol{k})$ na equação 4.8 .8 eliminamos diversos termos e obtemos

$$
\begin{aligned}
& \left\langle\Psi_{j \boldsymbol{k}^{\prime}}\left|\exp \left(\frac{i E_{j \boldsymbol{k}^{\prime}}}{\hbar} t\right) H^{\prime}\right| \Omega_{\boldsymbol{k}}(t)\right\rangle= \\
& \sum_{n, \boldsymbol{k}} a_{n \boldsymbol{k}}(t) e^{i \frac{E_{j \boldsymbol{k}^{\prime}-E_{n \boldsymbol{k}}}}{\hbar} t}\left\langle\Psi_{j \boldsymbol{k}^{\prime}}\left|H_{k p}+W_{k p}(\boldsymbol{k})+H_{e f}(\boldsymbol{k})\right| \Psi_{n \boldsymbol{k}}\right\rangle \\
& \quad=a_{j \boldsymbol{k}^{\prime}}(t) E_{j \boldsymbol{k}^{\prime}}+\sum_{n, \boldsymbol{k}} a_{n \boldsymbol{k}}(t) e^{i \frac{E_{j \boldsymbol{k}^{\prime}}-E_{n \boldsymbol{k}}}{\hbar} t}\left\langle\Psi_{j \boldsymbol{k}^{\prime}}\left|H_{e f}(\boldsymbol{k})\right| \Psi_{n \boldsymbol{k}}\right\rangle,
\end{aligned}
$$

de acordo com a definição

$$
\omega_{j \boldsymbol{k}^{\prime} ; n \boldsymbol{k}}:=\frac{E_{j \boldsymbol{k}^{\prime}}-E_{n \boldsymbol{k}}}{\hbar}
$$

As equações 4.8 .7 e 4.8.10 fornecem um sistema de equações diferenciais acopladas para os coeficientes $a_{n \boldsymbol{k}}(t)$,

$$
\frac{d a_{j \boldsymbol{k}^{\prime}}}{d t}(t)+\frac{i}{\hbar} \sum_{n, \boldsymbol{k}} a_{n \boldsymbol{k}}(t) e^{i \omega_{j \boldsymbol{k}^{\prime} ; n \boldsymbol{k}} t}\left\langle\Psi_{j \boldsymbol{k}^{\prime}}\left|H^{\prime}\right| \Psi_{n \boldsymbol{k}}\right\rangle=0 .
$$

Esta equação diferencial representa a variação dos coeficientes $a_{n \boldsymbol{k}}(t)$, quase como uma equação mestra***. Esta analogia então propõe as taxas de transições (da equação mestra) como sendo proporcionais aos diversos elementos de matriz $\left\langle\Psi_{j \boldsymbol{k}^{\prime}}\left|H^{\prime}\right| \Psi_{n \boldsymbol{k}}\right\rangle$. Mais adiante veremos como estas taxas podem ser escritas em termos destes elementos de matriz. Como está tudo bem definido, a solução da equação 4.8.12 (ou sua forma integral) é

$$
a_{j \boldsymbol{k}^{\prime}}(\tau)=a_{j \boldsymbol{k}^{\prime}}(0)-\frac{i}{\hbar} \sum_{n, \boldsymbol{k}}\left\langle\Psi_{j \boldsymbol{k}^{\prime}}\left|H^{\prime}\right| \Psi_{n \boldsymbol{k}}\right\rangle \int_{0}^{\tau} d t a_{n \boldsymbol{k}}(t) e^{i \omega_{j \boldsymbol{k}^{\prime} ; n \boldsymbol{k}} t}
$$

Esta equação integral é resolvida exatamente para alguns casos particulares, como sistemas

\footnotetext{
***A noção de probabilidade quântica e sistemas dinâmicos quânticos é um pouco diferente das definições
} usuais de probabilidade. Por isso, a analogia não é completamente direta. 
de dois níveis (o maser de amônia é o exemplo mais conhecido, discutido no terceiro volume da referência do famoso The Feynman Lectures ${ }^{94}$ ).

\subsubsection{Tratamento específico para as transições ópticas}

Uma das maiores diferenças entre a teoria semiclássica baseada no modelo de Bohr e a Mecânica Quântica está na impossibilidade de predizer a intensidade das linhas espectrais. Uma linha espectral representa uma transição, e sua intensidade é tão forte quanto mais frequente ocorrer esta transição. Como a Mecânica Quântica consegue estabelecer uma dinâmica para os processos de transição, é possível quantificar quão provável é cada transição. E isto é exatamente o que mostrarei nesta seção: em algum sentido, a intensidade das linhas espectrais de elétrons confinados num poço quântico.

Imaginando um elétron confinado no poço quântico, há quatro possíveis estados a serem ocupados para cada $\boldsymbol{k}_{\|}$. Antes de interagir com a luz, o sistema é regido pela hamiltoniana $H_{\text {eff }}$ e, portanto, esse elétron pode ser descrito por alguma combinação dos autoestados de $H_{\text {eff. }}$ Por simplicidade, considerarei que o elétron está no estado $\left|\boldsymbol{k} \lambda_{1} \lambda_{2}\right\rangle$. Em $t=0$ um feixe de fótons incide sobre o sistema e interage com este elétron. Como temos a hamiltoniana efetiva, que leva em conta efeitos da banda de condução, o sistema passa a ser constituído por um fóton e um elétron na banda de condução. Assim, o estado do sistema passa a ser

$$
\left|\boldsymbol{k}_{\| \lambda_{1}}^{\lambda_{2}} ; 1_{\boldsymbol{q} \boldsymbol{\pi}}\right\rangle=\left|\boldsymbol{k}_{\| \lambda_{1}}^{\lambda_{2}}\right\rangle \otimes\left|1_{\boldsymbol{q} \boldsymbol{\pi}}\right\rangle
$$

tal como defini na seção 4.2.2. Os operadores de criação e destruição só atuam nos estados de fótons, i.e., no estado $\left|1_{q \pi}\right\rangle$. Com a absorção deste fóton pelo elétron (confinado), o estado de fótons passa a ser o vácuo, $|0\rangle$; já o estado eletrônico passa a ser uma combinação linear dos outros autoestados de $H_{\text {eff }}$. Esta combinação pode ser determinada resolvendo uma equação como 4.8.13 se for possível o cálculo dos elementos de matriz.

Podemos escrever estas considerações propondo o seguinte ansatz:

$$
|\Omega(t)\rangle=\sum_{\varrho_{1}, \varrho_{2}, \boldsymbol{k}_{\|}} a_{\varrho_{1} \varrho_{2} \boldsymbol{k}_{\|}}(0)\left|\boldsymbol{k}_{\| \varrho_{1}}^{\varrho_{2}} ; 0\right\rangle .
$$

Note que $\boldsymbol{k}_{\|}$será mantido constante para todo tempo, i.e., só estamos investigando transições diretas $^{\dagger \dagger}$. Supomos que a absorção ocorre no tempo $t=0$. Isso significa que a condição

$\dagger^{\dagger} \dagger$ Não é difícil estender os cálculos para incluir transições indiretas, mas, como apresentaremos na seção 4.8.3, não fará muito sentido a menos que se investiguem aproximações que vão além da de dipolo elétrico (e.g., dipolo magnético). 
inicial 4.8 .5 é

$$
|\Omega(0)\rangle=\left|\boldsymbol{k}_{\| \lambda_{1}}^{\lambda_{2}} ; 1_{\boldsymbol{q} \pi}\right\rangle
$$

Comparando as equações 4.8.16 e 4.8.5, determinamos imediatamente a condição inicial da equação diferencial 4.8.12,

$$
a_{\varrho_{1} \varrho_{2} \boldsymbol{k}_{\| 1}^{\prime}}(0)=\delta_{\varrho_{1}, \lambda_{1}} \delta_{\varrho_{2}, \lambda_{2}} .
$$

Desta forma, a solução da equação 4.8 .12 pode ser colocado na forma integral

$$
a_{\varrho_{1} \varrho_{2} \boldsymbol{k}_{\|}}(\tau)=\delta_{\lambda_{1}^{\prime}, \lambda_{1}} \delta_{\lambda_{2}^{\prime}, \lambda_{2}}-\frac{i}{\hbar} \sum_{\lambda_{1} \lambda_{2} \boldsymbol{k}_{\|}} \int_{0}^{\tau} d t a_{\lambda_{1} \lambda_{2} \boldsymbol{k}_{\|}}(t) e^{i \omega_{j \boldsymbol{k}^{\prime} ; n \boldsymbol{k}} t}\left\langle\boldsymbol{k}_{\| \varrho_{1}}^{\varrho_{2}}\left|H^{\prime}\right| \boldsymbol{k}_{\| \lambda_{1}}^{\lambda_{2}}\right\rangle .
$$

Por fim, para quaisquer $\varrho_{1} \neq \lambda_{1}$ e $\varrho_{2} \neq \lambda_{2}$, obtemos

$$
a_{\varrho_{1} \varrho_{2} \boldsymbol{k}_{\|}}(\tau)=-\frac{i}{\hbar} \sum_{\varrho_{1} \varrho_{2} \boldsymbol{k}_{\|}} \int_{0}^{\tau} d t a_{\varrho_{1} \varrho_{2} \boldsymbol{k}_{\|}}(t) e_{\lambda_{1} \lambda_{2}}^{i \omega^{\varrho_{1} \varrho_{2}} t} \mathcal{M}_{\lambda_{1} \lambda_{2}}^{\varrho_{1} \varrho_{w}}(t),
$$

em que defini, para poupar um pouco de espaço,

$$
\mathcal{M}_{\lambda_{1} \lambda_{2}}^{\varrho_{1} \varrho_{w}}(t):=e^{-i \omega_{q} t} \sqrt{\frac{h c^{3}}{V \omega_{q}}}\left\langle\boldsymbol{k}_{\| \varrho_{1}}^{\varrho_{2}}\left|\frac{\hbar}{2 M} \boldsymbol{k} \cdot \boldsymbol{\pi}+\frac{\alpha_{M} e}{\hbar c} \boldsymbol{k} h_{w} \cdot \boldsymbol{\pi}-i \boldsymbol{\sigma} \cdot \boldsymbol{k} \tilde{\eta} \times \boldsymbol{\pi}\right| \boldsymbol{k}_{\| \lambda_{1}}^{\lambda_{2}}\right\rangle .
$$

Note que esta equação tem uma recorrência em $a_{\varrho_{1} \varrho_{2} \boldsymbol{k}_{\| 1}}(\tau)$ que torna o problema nada trivial. Uma primeira aproximação para esta equação é ${ }^{7}$

$$
a_{\varrho_{1} \varrho_{2} \boldsymbol{k}_{\|}}(\tau)=-\frac{i}{\hbar} \sum_{n, \boldsymbol{k}} \int_{0}^{\tau} d t e^{i \omega_{\lambda_{1} \lambda_{2}}^{\varrho_{1} \varrho_{2}} t} \mathcal{M}_{\lambda_{1} \lambda_{2}}^{\varrho_{1} \varrho_{w}}(t) .
$$

A parte temporal pode ser facilmente integrada, e continuarei a expor este assunto na seção 4.9.

Para maiores ordens, colocamos diferentes potências de $a_{\varrho_{1} \varrho_{2} \boldsymbol{k}_{11}}(\tau)^{79}$. Estas ordens geram termos que envolvem processos de múltimos fótons, absorções seguidas de reabsorções. Constitui uma notação especialmente útil para estas ordens mais altas os diagramas de Feynman.

\subsubsection{Diagramas de Feynman: interações de um fóton}

Em primeira ordem, os termos proporcionais a $\boldsymbol{A}$ envolvem operadores de criação e destruição, enquanto que $A^{2}$ envolve produto deles. Isso significa que os únicos eventos que poderemos contemplar com $\boldsymbol{A}$, nesta ordem, são de emissão ou absorção. Estes eventos são descritos pelos seguintes diagramas: 

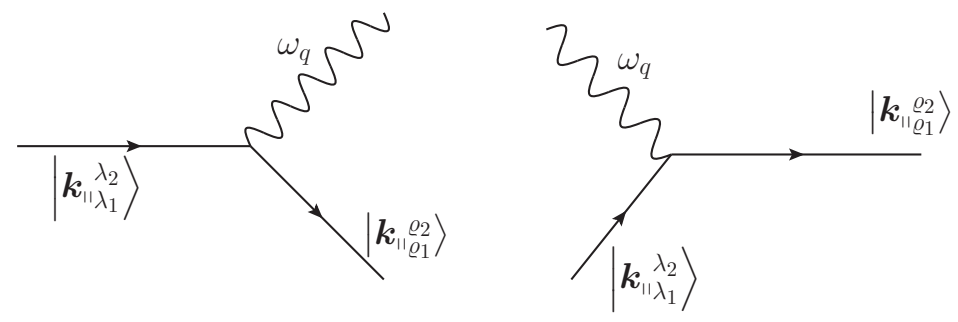

Estes são os processos mais simples e, em geral, mais fortes. Outras ordens costumam ser correções importantes, mas menos fortes (e.g., espectroscopia Raman).

O termo com $A^{2}$ está relacionado, mesmo em primeira ordem, com processos de dois fótons. São exemplos destes processos os seguintes diagramas:
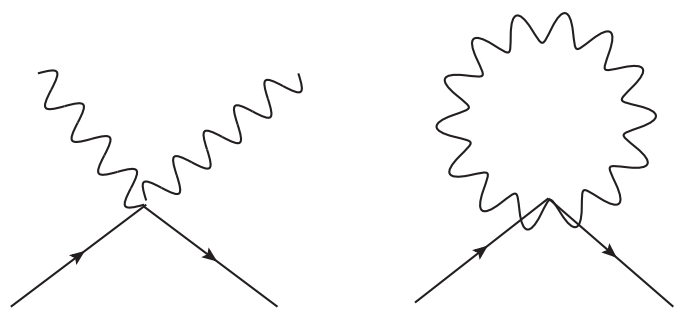

Um dos processos mostrados acima não envolve exatamente dois fótons, mas a absorção imediatamente após a emissão de um mesmo fóton.

Passarei a focar nossa discussão nos processos de um fóton. Por este motivo, deixaremos de lado, no capítulo seguinte, o termo proporcional a $A^{2}$. Trabalhos futuros interessantíssimos envolvem somar diversos diagramas para ordens mais altas.

\subsubsection{Aproximação de dipolo elétrico e transições indiretas}

Para um gás de elétrons, em alguma dimensão (seja um ponto, fio, poço ou até mesmo um volume quântico), então parte da função de onda pode ser descrita como a de elétrons livres. Se $x$ for uma coordenada em que o elétron está livre, a função de onda pode ser separada como $\psi(\boldsymbol{r})=\psi_{0}\left(\boldsymbol{r}^{\prime}\right) \phi(x)$, em que $\boldsymbol{r}^{\prime}$ não depende de $x$ mas apenas das outras variáveis. Neste caso, $\phi(x)=e^{i k x}$, em que o $k$ assume valores de acordo com as condições de contorno aplicadas. Verificamos isto na prática durante nossas discussões do capítulo 3, quando verificamos que a equação efetiva 3.1.1 era separável em $x$ e $y$. Ao final do capítulo, apresentamos a solução numa forma similar a

$$
\left\langle\boldsymbol{r} \mid \boldsymbol{k}_{\| \lambda_{1}}^{\lambda_{2}}\right\rangle=e^{i \boldsymbol{k}_{\|} \cdot \boldsymbol{r}} f(z)\left|\lambda_{1}\right\rangle
$$

Este tipo de solução recorre em fios quânticos, mas para apenas uma das direções. 


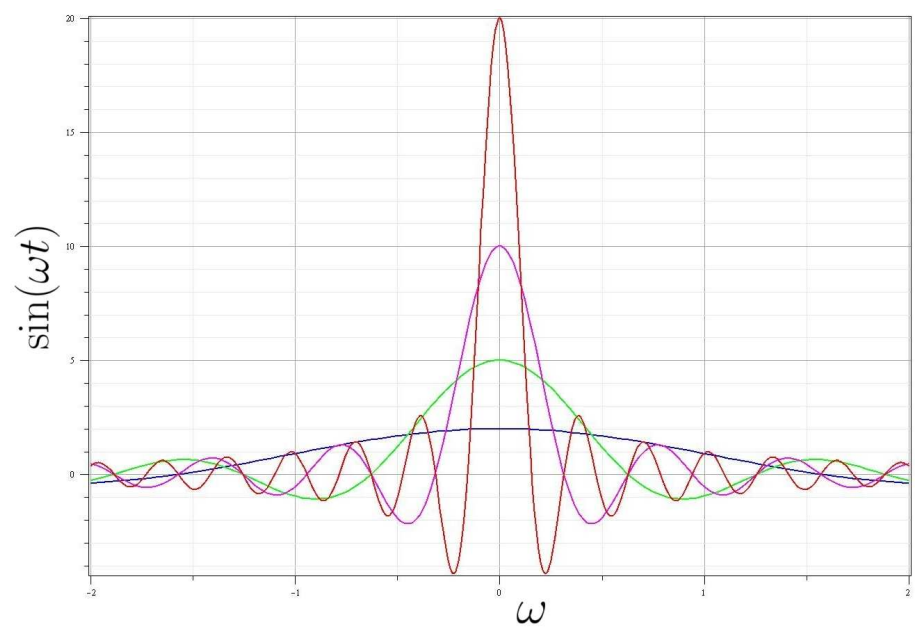

Figura 4.3 - A taxa de transição que calculamos representa assintoticamente uma distribuição de Dirac para a diferença $\omega_{q}-\omega_{\lambda_{1} \lambda_{2}}^{\varrho_{1}}$. Na figura, vemos o gráfico da dependência nesta diferença de energias para diferentes tempos. Conforme $t$ cresce, a altura cresce linearmente e a largura decresce por um fator $\pi / t$.

Quando calculamos elementos de matriz entre estados eletrônicos com diferentes vetores de onda, digamos, $\boldsymbol{k}_{\|}$e $\boldsymbol{k}_{\|}^{\prime}$, acabamos com integrais como

$$
\int d x e^{i k x} \frac{\partial}{\partial x} e^{i k^{\prime} x}=k^{\prime} \int d x e^{i k x} e^{i k^{\prime} x}=0, \quad k \neq k^{\prime}
$$

Isso vale apenas para a aproximação de dipolo elétrico. Assim, não há a possibilidade de troca de momento via transições ópticas. Este fato vincula fortemente a aproximação de dipolo elétrico com o que chamamos de transições diretas, i.e., transições em que o momento do elétron no gás não se altera.

\subsection{Transições Ópticas usando Regra de Ouro}

Para tempos muito longos, é possível derivar uma fórmula mais compacta e que o significado físico é bem claro. Para tal, teremos que calcular o valor assintótico da integral no tempo da equação 4.8.21. Resolver esta integral não é complicado, o que resulta em

$$
a_{\varrho_{1} \varrho_{2} \boldsymbol{k}_{\|}}(\tau)=\frac{\mathcal{M}(0)}{i \hbar} \frac{e^{i\left(\omega_{q}-\omega_{\lambda_{1} \lambda_{2}}^{\varrho_{1} \varrho_{2}}\right) \tau}+1}{i\left(\omega_{q}-\omega_{\lambda_{1} \lambda_{2}}^{\varrho_{1} \varrho_{2}}\right)}
$$


A probabilidade de o elétron transicionar para o estado $\left|\boldsymbol{k}_{\| \varrho_{1}}^{\varrho_{2}}\right\rangle$ é simplesmente $\left|a_{\varrho_{1} \varrho_{2} \boldsymbol{k}_{\|}}(\tau)\right|^{2}$. A taxa com que essa transição ocorre pode ser calculada como

$$
\mathcal{W}(\tau):=\frac{d}{d \tau}\left|a_{\varrho_{1} \varrho_{2} \boldsymbol{k}_{\|}}(\tau)\right|^{2}=\frac{2|\mathcal{M}(0)|^{2}}{\hbar^{2}} \frac{\sin \left(\frac{\omega_{q}-\omega_{\lambda_{1} \lambda_{2}}^{\varrho_{1}}}{2} \tau\right)}{\frac{\omega_{q}-\omega_{\lambda_{1} \lambda_{2}}^{\varrho_{1} \varrho_{2}}}{2}} .
$$

A dependência temporal é uma das representações integrais da distribuição de Dirac se fizemos $\tau \rightarrow \infty$ (veja a figura 4.3). Portanto, a taxa de transição tem o seguinte comportamento assintótico:

$$
\mathcal{W} \sim \frac{2 \pi}{\hbar^{2}}|\mathcal{M}(0)|^{2} \delta\left(\omega_{q}-\omega_{\lambda_{1} \lambda_{2}}^{\varrho_{1} \varrho_{2}}\right)
$$

Esta última expressão é usualmente conhecida como regra de ouro de Fermi ${ }^{84-86}$. Usaremos esta expressão para quantificar a intensidade de cada uma das transições. Muitas vezes, $1 / \mathcal{W}$ representa o tempo característico para ocorrência da transição. Note que este mesmo argumento pode ser utilizado para um vasto conjunto de perturbações, que mantêm em comum apenas a dependência temporal do tipo $e^{ \pm i \omega t}$ ( $\omega$ como uma constante específica).

Segundo a expressão 4.9.3 para as taxas de transições, apenas as transições ressonantes (i.e., em que a energia cedida ao sistema é exatamente a diferença de energia entre dois quaisquer estados do sistema) são observadas a tempos longos. As transições não ressonantes devem desaparecer logo por dissipação da energia. Por isso, $\delta\left(\omega_{q}-\omega_{\lambda_{1} \lambda_{2}}^{\varrho_{1} \varrho_{2}}\right)$ representa a conservação de energia

$$
\hbar \omega_{q}=\left|E_{\varrho_{1} \varrho_{2}}-E_{\lambda_{1} \lambda_{2}}\right| .
$$

O sinal da diferença $E_{\varrho_{1} \varrho_{2}}-E_{\lambda_{1} \lambda_{2}}$ indica apenas se o processo é de absorção ou de emissão. Essas diferenças de energia ficam sempre no infravermelho (principalmente, no infravermelho próximo) para a maioria dos materiais.

\subsubsection{Matriz óptica}

Na aproximação de dipolo elétrico, usualmente (em sistemas atômicos, por exemplo) a perturbação que age sobre o sistema fica proporcional ao vetor $\boldsymbol{P}$. Assim, para uma transição de um estado $|A\rangle$ a um estado $|B\rangle$, a regra de ouro fornece como taxa

$$
\mathcal{W} \propto|\langle B|\boldsymbol{P} \cdot \boldsymbol{\pi}| A\rangle|^{2}
$$

Como em geral esta aproximação vale muito bem para frequências dentro do visível, é comum encontrar referências ao operador $\boldsymbol{P} \cdot \boldsymbol{\pi}$ como matriz óptica. O resultado da interação $\boldsymbol{P} \cdot \boldsymbol{\pi}$ para a banda de condução foi uma interação $\mathcal{W}_{\text {eff }}$. Podemos então chamar 4.8.20 de matriz 
óptica efetiva. No capítulo seguinte, concentrarei nossa atenção em calcular os elementos desta matriz óptica efetiva e calcular as taxas de transições entre estados do poço quântico.

Em outras referências, como o trabalho de Yang et al ${ }^{91}$, o procedimento difere substancialmente do que estou empregando aqui. Em vez de obter uma interação elétron-fóton efetiva para a banda de condução, as bandas de valência voltam para o jogo e eles calculam os elementos de matriz de $\boldsymbol{P} \cdot \boldsymbol{\pi}$ usando vetores de oito componentes. Eles não observam troca de spin por tratarem as componentes da banda de condução como independentes, e assim o acoplamento spin-órbita desaparece. Fora isso, ambos os procedimentos são equivalentes. Apresentei ${ }^{81,93,95}$ estas diferenças como tema de painéis para discutir possíveis modelagens para a interação elétron-fóton na banda de condução de poços quânticos. 


\subsection{Revisão}

Demonstrei que é lícito realizar o acoplamento mínimo na hamiltoniana efetiva que obtivemos no capítulo 2. Incluindo campo de radiação, a equação efetiva para a banda de condução fica

$$
\left[H_{\mathrm{eff}}+W_{\mathrm{eff}}-\mu_{B}\left(g_{1}+g_{2}\right) \boldsymbol{\sigma} \cdot \mathcal{B}\right] \psi_{S}=\left(\epsilon-V-h_{6}\right) \psi_{S}
$$

em que

$$
H_{\mathrm{eff}}=\frac{\hbar^{2}}{2 M} k^{2}+h_{6}+V+V_{\text {so }}+V_{d}
$$

e

$$
W_{\mathrm{eff}}=\frac{\hbar}{2 M} \boldsymbol{k} \cdot \boldsymbol{A}+\frac{\alpha_{M} e}{\hbar c} \boldsymbol{k} h_{w} \cdot \boldsymbol{A}-\frac{i e}{\hbar c} \boldsymbol{\sigma} \cdot \boldsymbol{k} \tilde{\eta} \times \boldsymbol{A}+\frac{e^{2}}{2 M c^{2}} A^{2},
$$

em que definimos

$$
\tilde{\eta}=\gamma_{H} V+\gamma_{e} h_{w}
$$

A constante giromagnética, induzida pelas bandas de valência, é

$$
g_{1}+g_{2}=\frac{\mathcal{P}^{2}}{3 \mu_{B}}\left[\gamma_{1}+\tilde{\eta}\right] .
$$

Reutilizamos as definições e constantes a seguir:

$$
\begin{aligned}
\frac{\hbar^{2}}{2 M} & =\frac{\hbar^{2}}{2 m}+\frac{\mathcal{P}^{2}}{3} \gamma_{1}+\frac{\mathcal{P}^{2}}{3}\left[\gamma_{2}^{\prime}+\gamma_{2} \delta_{6}\right] h_{w} \\
V_{d} & =-\frac{\mathcal{P}^{2}}{6} \gamma_{2} k^{2}\left(h_{6}+V\right)-\frac{\mathcal{P}^{2}}{3}\left[\gamma_{2}^{\prime}+\gamma_{2} \delta_{6}\right]\left(\boldsymbol{k} h_{w}\right) \cdot \boldsymbol{k} \\
V_{s o} & =-\frac{\mathcal{P}^{2}}{3} i \boldsymbol{\sigma} \cdot\left\{\eta_{H}(\boldsymbol{k} V)-\eta_{e}\left(\boldsymbol{k} h_{w}\right)\right\} \times \boldsymbol{k} \\
\gamma_{1} & =\frac{2}{E_{g}}+\frac{1}{E_{g}+\Delta_{g}} \\
\gamma_{2} & =\frac{2}{E_{g}^{2}}+\frac{1}{\left(E_{g}+\Delta_{g}\right)^{2}} \\
\gamma_{2}^{\prime} & =\frac{2 \delta_{8}}{E_{g}^{2}}+\frac{\delta_{7}}{\left(E_{g}+\Delta_{g}\right)^{2}} \\
\eta_{H} & =\frac{1}{E_{g}^{2}}-\frac{1}{\left(E_{g}+\Delta_{g}\right)^{2}} \\
\eta_{e} & =\frac{\delta_{8}}{E_{g}^{2}}-\frac{\delta_{7}}{\left(E_{g}+\Delta_{g}\right)^{2}}
\end{aligned}
$$




\section{CAPÍTULO 5}

\section{Taxas de Transições}

In the city of fallen angels, where the ocean meets the sand

You will form a strong alliance and the world's most awesome band

Dio, em Tenacious D - Kickapoo, 2007.

Calculo neste capítulo os elementos de matriz dos acoplamentos elétron-fóton usando a aproximação de dipolo elétrico. Vou focar o assunto no acoplamento assistido por spin. Mostrarei que ele possibilita o spin flip dentro da banda de condução. Mostrarei também um efeito de saturação neste acoplamento que não ocorre no usual $\boldsymbol{P} \cdot \boldsymbol{A}$.

\subsection{Frequências características}

Ao final do capítulo anterior, vimos que as transições que sobrevivem a tempos longos são aquelas cuja diferença de energias entre o estado final e inicial iguala-se à energia do fóton que provocou tal transição. Este é o caso de "transições ressonantes", as mais importantes e mais fortes. Isso porque o estado final é um estado estacionário, e o sistema tende a permanecer neste estado - ao menos até que flutuações (por exemplo, do vácuo) o façam voltar a um estado de menor energia.

Entre os quatro estados eletrônicos disponíveis, podemos enumerar seis transições (absorções ou emissões). As frequências correspondentes a estas transições são tipicamente na região conhecida como o infravermelho próximo*. Na figura 5.1(a), mostro a dependência em $k_{\|}$para simulações feitas com $\mathrm{Al}_{0.2} \operatorname{In}_{0.8} S b / \operatorname{In} S b$. Todas elas estão na ordem de $T H z$, como comentei anteriormente.

*Do inglês, Near Infrared (NIR). 


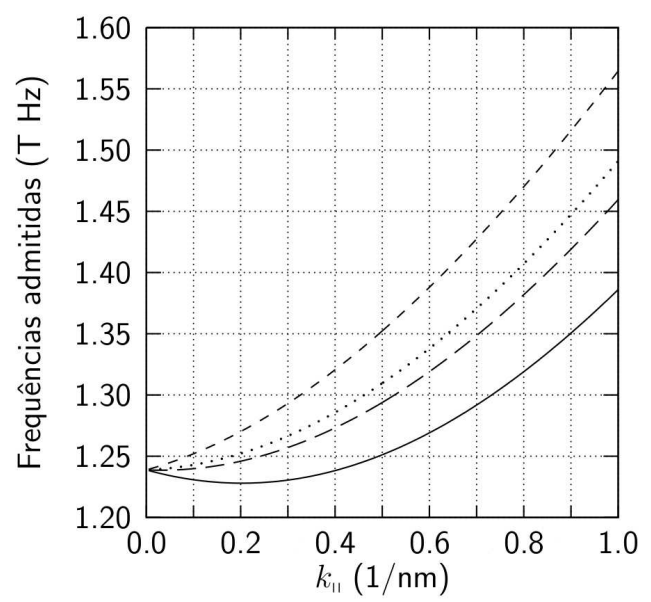

(a) $A l_{0.2} \operatorname{In}{ }_{0.8} S b / \operatorname{InSb}$

\section{$\mid 1,1>$}

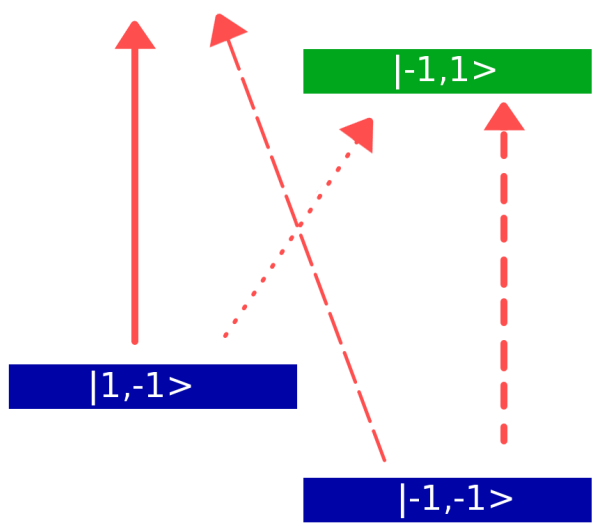

(b) Transições pictóricas

Figura 5.1 - Frequências admitidas para quatro possíveis transições ressonantes em função de $k_{\|}$. $\mathrm{Na}$ representação pictórica (b) das transições entre os estados, a forma das flechas coincidem com a forma das curvas no gráfico. Estes dados foram tirados para o poço $A l_{0.2} I_{0.8} S b / I n S b$ com um pequeno gate aplicado para quebrar degenerescência de spin.

\subsection{Elementos da matriz óptica}

Das interações 4.7.18, vamos usar os acoplamentos

$$
W_{\mathrm{eff}}^{\prime}=\frac{\hbar}{2 M} \boldsymbol{k} \cdot \boldsymbol{A}+\frac{\alpha_{M} e}{\hbar c} \boldsymbol{k} h_{w} \cdot \boldsymbol{A}-\frac{i e}{\hbar c} \boldsymbol{\sigma} \cdot \boldsymbol{k} \tilde{\eta} \times \boldsymbol{A},
$$

em que deixei de lado o acoplamento $\left(e^{2} / 2 M c^{2}\right) A^{2}$ intensionalmente por estarmos interessados apenas em processos de um fóton e em primeira ordem de perturbação (veja a seção 4.8.2). Como discuti amplamente na seção 4.9, a taxa de ocorrência de uma transição entre dois dados estados depende do elemento de matriz de $W_{\text {eff }}^{\prime}$. Como cada termo da equação 5.2.1 tem suas próprias características, vou apresentá-los separadamente.

Os elementos de matriz são calculados usando os autovetores apresentados na seção 3.6. Podemos usar as soluções do caso assimétrico (mais geral), e fazermos $\eta_{j j}=0$ no momento em que formos estudar especificamente o caso simétrico. Podemos escrever estes autoestados na forma

$$
\left|\boldsymbol{k}_{\| \lambda_{1}}^{\lambda_{2}}\right\rangle=e^{i \boldsymbol{k}_{\|} \cdot \boldsymbol{r}} A_{\lambda_{1} \lambda_{2}}^{\boldsymbol{k}_{\|}}\left(B_{\lambda_{1} \lambda_{2}}^{\boldsymbol{k}_{\|}}\left|u_{0}\right\rangle+\left|u_{1}\right\rangle\right)\left|\lambda_{1}\right\rangle
$$

em que

$$
B_{\lambda_{1} \lambda_{2}}^{\boldsymbol{k}_{\|}}:=\frac{\eta_{01}\left(k_{\|}-k_{0}\right)}{\lambda_{2} \Omega_{\lambda_{1}}(k)+\left(\widetilde{\epsilon}_{-}+\lambda_{1} \alpha_{-}\right)}
$$


e as demais definições da seção 3.6.2. A grandeza

$$
A_{\lambda_{1} \lambda_{2}}^{\boldsymbol{k}_{\text {un }}}:=\frac{1}{\sqrt{\left(B_{\lambda_{1} \lambda_{2}}^{\boldsymbol{k}_{\|}}\right)^{2}+1}}
$$

apenas certifica a normalização de $\left|\boldsymbol{k}_{\|} \lambda_{1} \lambda_{2}\right\rangle$. A relação de dispersão 3.5 .6 destes estados será usada para determinar corretamente as frequências de ressonância que incitam as transições que estudaremos.

Como vimos que os processos de absorção e emissão têm mesma probabilidade de ocorrer, vou restringir mais ainda esta discussão: calcularei os elementos de matriz da absorção de um fóton de frequência $\omega_{q}$ e polarização $\boldsymbol{\pi}$. Formularei um método geral para o cálculo dos elementos de matriz usando a transição

$$
\left|\boldsymbol{k}_{\| \lambda_{1}}^{\lambda_{2}} ; 1_{\boldsymbol{q} \boldsymbol{\pi}}\right\rangle \rightarrow\left|\boldsymbol{k}_{\| \varrho_{1}}^{\varrho_{2}} ; 0\right\rangle
$$

Isto significa o "desaparecimento" de um fóton e a mudança do estado eletrônico $\left|\boldsymbol{k}_{\|}{ }_{\lambda_{1}}^{\lambda_{2}}\right\rangle \rightarrow$ $\left|\boldsymbol{k}_{\|} \varrho_{1}\right\rangle$. Vale lembrar que o vetor $\boldsymbol{k}_{\|}$não se altera na transição por estarmos trabalhando na aproximação de dipolo elétrico (veja seção 4.8.3). Das interações da equação 5.2.1, os únicos operadores que atuam nos estados de fótons vêm do potencial vetor. Para qualquer uma das interações, o elemento de matriz separa-se em duas partes: a parte referente ao orbital eletrônico e a parte referente ao estado de fóton. Ao efetuar estes cálculos, vemos que

$$
\left\langle 0|\boldsymbol{A}| 1_{\boldsymbol{q} \boldsymbol{\pi}}\right\rangle=\sqrt{\frac{2 \pi \hbar c^{3}}{V}} \sum_{\boldsymbol{q} \lambda} \frac{\boldsymbol{\pi}}{\sqrt{\omega_{q}}} e^{-i \omega_{\boldsymbol{q}} t} .
$$

Este fator será presente em todos os elementos e será responsável pela validade da regra de ouro de Fermi.

Com a quebra de simetria translacional criada pelo poço quântico, a direção $\hat{z}$ será tratada em separado. Podemos separar a polarização da mesma forma: $\boldsymbol{\pi}=\boldsymbol{\pi}_{\|}+\pi_{z} \hat{z}$, em que $\boldsymbol{\pi}_{\|}$é a componente do vetor polarização no gás bidimensional confinado no poço.

\subsubsection{Acoplamento usual}

Como este é o acoplamento mais forte, começarei os cálculos com este acoplamento,

$$
W_{p \cdot a}=\frac{\hbar}{2 M} \boldsymbol{k} \cdot \boldsymbol{A},
$$


em que $M$ é a massa efetiva 4.7.19a. Usando a expressão quantizada de $\boldsymbol{A}$, dada na equação 4.2.16, já na aproximação de dipolo elétrico, temos

$$
W_{p \cdot a}=\sum_{\boldsymbol{q} \lambda} \frac{\mathcal{C}}{\sqrt{\omega_{q}}} \boldsymbol{k} \cdot \boldsymbol{\pi}\left(a_{\boldsymbol{q} \lambda} e^{-i \omega_{\boldsymbol{q}} t}+a_{\boldsymbol{q} \lambda}^{\dagger} e^{i \omega_{\boldsymbol{q}} t}\right),
$$

em que defini

$$
\mathcal{C}:=\frac{\hbar}{2 M} \sqrt{\frac{2 \pi \hbar c^{3}}{V}}
$$

O primeiro termo em parênteses da equação 5.2 .6 representa processos de absorção; o segundo, emissão.

Calculando o elemento de matriz para absorção,

$$
\left\langle\boldsymbol{k}_{\| \varrho_{1}}^{\varrho_{2}} ; 0\left|W_{p \cdot a}\right| \boldsymbol{k}_{\| \lambda_{1}}^{\lambda_{2}} ; 1_{q \pi}\right\rangle=\frac{\mathcal{C}}{\sqrt{\omega_{q}}} \boldsymbol{\pi}_{\boldsymbol{q} \boldsymbol{\lambda}} \cdot\left\langle\boldsymbol{k}_{\| \varrho_{1}}^{\varrho_{2}}|\boldsymbol{k}| \boldsymbol{k}_{\| \lambda_{1}}^{\lambda_{2}}\right\rangle
$$

Portanto, precisamos apenas calcular o vetor $\left\langle\boldsymbol{k}_{\| \varrho_{1}}^{\varrho_{2}}|\boldsymbol{k}| \boldsymbol{k}_{\| \lambda_{1}}^{\lambda_{2}}\right\rangle$. Separei este elemento de matriz em duas partes,

$$
\boldsymbol{\pi} \cdot\left\langle\boldsymbol{k}_{\| \varrho_{1}}^{\varrho_{2}}|\boldsymbol{k}| \boldsymbol{k}_{\| \lambda_{1}}^{\lambda_{2}}\right\rangle=\boldsymbol{\pi}_{\|} \cdot\left\langle\boldsymbol{k}_{\varrho_{1}}^{\varrho_{2}}\left|\boldsymbol{k}_{\|}\right| \boldsymbol{k}_{\| \lambda_{1}}^{\lambda_{2}}\right\rangle+\pi_{z}\left\langle\boldsymbol{k}_{\| \varrho_{1}}^{\varrho_{2}}\left|k_{z}\right| \boldsymbol{k}_{\| \lambda_{1}}^{\lambda_{2}}\right\rangle
$$

Quanto à polarização no plano, vale lembrar que o produto $\boldsymbol{\pi}_{\|} \cdot \boldsymbol{k}=\pi_{x} k_{z}+p_{y} k_{y}$, já que $\boldsymbol{\pi}_{\|}$ não tem componente em $\hat{z}$. Assim, usando a forma 5.2.2 para os autoestados,

$$
\left\langle\boldsymbol{k}_{\| \varrho_{1}}^{\varrho_{2}}\left|\boldsymbol{k}_{\|}\right| \boldsymbol{k}_{\| \lambda_{1}}^{\lambda_{2}}\right\rangle=\boldsymbol{k}_{\|}\left\langle\boldsymbol{k}_{\| \varrho_{1}}^{\varrho_{2}} \mid \boldsymbol{k}_{\| \lambda_{1}}^{\lambda_{2}}\right\rangle .
$$

No entanto, os autoestados $\left|\boldsymbol{k}_{\| \varrho_{1}}^{\varrho_{2}}\right\rangle$ e $\left|\boldsymbol{k}_{\| \lambda_{1}}^{\lambda_{2}}\right\rangle$ são ortogonais. Portanto, não há contribuições de $\boldsymbol{\pi}_{\|}$para este acoplamento. Quanto à parte fora do plano, ficamos com

$$
\begin{aligned}
& \left\langle\boldsymbol{k}_{\| \varrho}^{\varrho_{2}}\left|k_{z}\right| \boldsymbol{k}_{\| \lambda_{1}}^{\lambda_{2}}\right\rangle=\pi_{z} \delta_{\varrho_{1}, \lambda_{1}} A_{\lambda_{1} \lambda_{2}}^{\boldsymbol{k}_{\|}} A_{\varrho 1 \varrho_{2}}^{\boldsymbol{k}_{\|}}\left(B_{\varrho 1 \varrho_{2}}^{\boldsymbol{k}_{\|}} B_{\lambda_{1} \lambda_{2}}^{\boldsymbol{k}_{\|}}\left\langle u_{0}\left|k_{z}\right| u_{0}\right\rangle+\right. \\
& \left.B_{\varrho_{1} \varrho_{2}}^{\boldsymbol{k}_{\varrho_{1}}}\left\langle u_{0}\left|k_{z}\right| u_{1}\right\rangle+B_{\lambda_{1} \lambda_{2}}^{\boldsymbol{k}_{n_{1}}}\left\langle u_{1}\left|k_{z}\right| u_{0}\right\rangle+\left\langle u_{1}\left|k_{z}\right| u_{1}\right\rangle\right) .
\end{aligned}
$$

Ainda assim não há troca de spin nestas transições. Além disso, minhas simulações numéricas mostram que os elementos de matriz $\left\langle u_{j}\left|k_{z}\right| u_{\ell}\right\rangle$ são todos praticamente nulos. Isto significa apenas que para os poços que estamos estudando, e para as primeiras sub-bandas, a contribuição $\boldsymbol{P} \cdot \boldsymbol{A}$ não contribui (significativamente) para as transições ópticas. Em outros poços (e.g., de tipo II ou $I I I$ ) pode ocorrer, no entanto, resultados bem diferentes.

Em diversos momentos da literatura, modelos de uma banda ${ }^{\dagger}$ mostram que transições

${ }^{\dagger}$ O modelo que usamos durante todo o trabalho foca-se apenas uma banda (a de condução, desdobrada em duas com spin). No entanto, um modelo de uma banda refere-se a desprezar completamente o acoplamento desta com as outras bandas. 
intersub-bandas só ocorrem com polarização na direção do eixo de crescimento, ou seja, $\boldsymbol{\pi}=$ $\pi \hat{z}$. Na equação 5.2.12, vemos uma confirmação para esta afirmação. Modelos multibandas colocaram este resultado a prova ${ }^{55,96}$ e mostraram que na verdade há sim a possibilidade de haver transição mesmo com $\pi_{z}=0{ }^{95}$. Como o acoplamento $\boldsymbol{k} \cdot \boldsymbol{A}$ vem primordialmente da própria banda de condução (a menos da renormalização da massa), este é exatamente o único tipo de interação que ocorre em um modelo de uma banda. Na seção 5.2.3, mostrarei ${ }^{81}$ um acoplamento que é originado das bandas de valência e mostraremos que de fato a polarização in plane também induz transições ópticas. Além disso, ao final, veremos que apenas esta polarização é capaz de induzir spin flip.

\subsubsection{Caso simétrico}

Quando o potencial estrutural é simétrico, então os acoplamentos spin-órbita intersubbandas (Rashba) são nulos. Além disso, $\left|u_{0}\right\rangle$ é par e $\left|u_{1}\right\rangle$, impar. Note que isso não significa que $\left|\boldsymbol{k}_{\| \lambda_{1}}^{\lambda_{2}}\right\rangle$ tenha paridade definida.

Pelas paridades definidas dos $\left|u_{0}\right\rangle$ e $\left|u_{1}\right\rangle$, donde decorre

$$
\left\langle u_{j}\left|k_{z}\right| u_{j}\right\rangle=0 \quad \text { e } \quad\left\langle u_{0}\left|k_{z}\right| u_{1}\right\rangle \neq 0
$$

Assim, a equação 5.2 .12 pode ser simplificada em

$$
\left\langle\boldsymbol{k}_{\| \varrho_{1}}^{\varrho_{2}}\left|k_{z}\right| \boldsymbol{k}_{\| \lambda_{1}}^{\lambda_{2}}\right\rangle=\delta_{\varrho_{1}, \lambda_{1}}\left(B_{\varrho_{1} \varrho_{2}}^{\boldsymbol{k}_{\|}}\left\langle u_{0}\left|k_{z}\right| u_{1}\right\rangle+B_{\lambda_{1} \lambda_{2}}^{\boldsymbol{k}_{\|}}\left\langle u_{1}\left|k_{z}\right| u_{0}\right\rangle\right) .
$$

\subsubsection{Acoplamento radiação-momento-campo elétrico}

Vamos analisar brevemente como são os elementos de matriz do acoplamento

$$
\begin{gathered}
W_{s}:=\frac{\alpha_{M} e}{\hbar c} \boldsymbol{k} h_{w} \cdot \boldsymbol{A}= \\
-i\left[\delta\left(z-a_{0}\right)-\delta\left(z+a_{0}\right)\right] \sum_{\boldsymbol{q} \lambda} \frac{\mathcal{C}_{s}}{\sqrt{\omega_{q}}} \hat{z} \cdot \boldsymbol{\pi}_{\boldsymbol{q} \lambda}\left(e^{-i \omega_{\boldsymbol{q}} t}+a_{\boldsymbol{q} \lambda}^{\dagger} e^{i \omega_{\boldsymbol{q}} t}\right),
\end{gathered}
$$

em que usei a definição

$$
\mathcal{C}_{s}:=\frac{e \alpha_{M}}{\hbar c} \sqrt{\frac{2 \pi \hbar c^{3}}{V}}
$$

A única parte que dificulta o cálculo dos elementos de matriz são as deltas de Dirac,

$$
\delta\left(z-a_{0}\right)-\delta\left(z+a_{0}\right)
$$


Mesmo assim, é fácil ver que

$$
\begin{aligned}
& \left\langle\boldsymbol{k}_{\| \varrho_{1}}^{\varrho_{2}}\left|\delta\left(z-a_{0}\right)-\delta\left(z+a_{0}\right)\right| \boldsymbol{k}_{\| \lambda_{1}}^{\lambda_{2}}\right\rangle= \\
& A_{\lambda_{1} \lambda_{2}}^{\boldsymbol{k}_{\|}} A_{\lambda_{1} \lambda_{2}}^{\boldsymbol{k}_{\mathrm{N}^{\prime}}}\left[B_{\varrho 1 \varrho_{2}}^{\boldsymbol{k}_{\|}} B_{\lambda_{1} \lambda_{2}}^{\boldsymbol{k}_{\|}} u_{0}^{2}\left(a_{0}\right)+u_{1}^{2}\left(a_{0}\right)+u_{0}\left(a_{0}\right) u_{1}\left(a_{0}\right)\left(B_{\varrho 1 \varrho_{2}}^{\boldsymbol{k}_{\|}}+B_{\lambda_{1} \lambda_{2}}^{\boldsymbol{k}_{\|}}\right)-\right. \\
& \left.B_{\varrho_{1} \varrho_{2}}^{\boldsymbol{k}_{\varrho_{1}}} B_{\lambda_{1} \lambda_{2}}^{\boldsymbol{k}_{\boldsymbol{k}^{\prime}}} u_{0}^{2}\left(-a_{0}\right)-u_{1}^{2}\left(-a_{0}\right)-u_{0}\left(-a_{0}\right) u_{1}\left(-a_{0}\right)\left(B_{\varrho_{1} \varrho_{2}}^{\boldsymbol{k}_{\boldsymbol{N}^{\prime}}}+B_{\lambda_{1} \lambda_{2}}^{\boldsymbol{k}_{\|}}\right)\right] .
\end{aligned}
$$

A única dependência em $\boldsymbol{k}_{\|}$está nos termos com $B_{\lambda_{1} \lambda_{2}}^{\boldsymbol{k}_{\|}}$e $B_{\varrho_{1} \varrho_{2}}^{\boldsymbol{k}_{\|}}$. No caso simétrico, podemos ainda simplificar um pouco o resultado anterior:

$$
\left\langle\boldsymbol{k}_{\| \varrho_{1}}^{\varrho_{2}}\left|\delta\left(z-a_{0}\right)-\delta\left(z+a_{0}\right)\right| \boldsymbol{k}_{\| \lambda_{1}}^{\lambda_{2}}\right\rangle=2 u_{1}\left(a_{0}\right) u_{0}\left(a_{0}\right)\left(B_{\varrho_{1} \varrho_{2}}^{\boldsymbol{k}_{\|}}+B_{\lambda_{1} \lambda_{2}}^{\boldsymbol{k}_{\|}}\right) .
$$

Note que este acoplamento não fornece spin flip e só depende de $\pi_{z}$ (tanto no caso simétrico como no caso assimétrico). Portanto, podemos certamente afirmar que não há contribuição para spin flip com polarização paralela à direção de crescimento. A única forma de induzir spin flip é utilizando o acoplamento assistido por spin, que depende apenas de $\boldsymbol{k}_{\|}$.

\subsubsection{Acoplamento assistido por spin}

Escreverei este acoplamento na forma

$$
W_{\sigma}=-i \boldsymbol{\sigma} \cdot \boldsymbol{k} \tilde{\eta} \times \boldsymbol{A}=-\sum_{\boldsymbol{q} \lambda} \frac{\mathcal{C}_{\sigma}}{\sqrt{\omega_{q}}} \boldsymbol{\sigma} \cdot(\eta \hat{z}) \times \boldsymbol{\pi}_{\boldsymbol{q} \lambda}\left(a_{\boldsymbol{q} \lambda} e^{-i \omega_{\boldsymbol{q}} t}+a_{\boldsymbol{q} \lambda}^{\dagger} e^{i \omega_{\boldsymbol{q}} t}\right)
$$

em que já usei, por comodidade, a definição $\eta=i \boldsymbol{k} \tilde{\eta}$. Assim como o acoplamento spinórbita que estudamos no capítulo 2 , este acoplamento origina-se da banda de valência. Defini também

$$
\mathcal{C}_{\sigma}:=\sqrt{\frac{2 \pi \hbar c^{3}}{V}}
$$

Simplificando o produto vetorial, temos

$$
\boldsymbol{\sigma} \cdot \hat{z} \times \boldsymbol{\pi}=\pi_{x} \sigma_{y}-\pi_{y} \sigma_{x}
$$

o que lembra muito o acoplamento spin-órbita se trocarmos $\pi_{j}$ por $k_{j}$ (reveja a seção 3.3). As soluções $\left|\boldsymbol{k}_{\| \lambda_{1}}^{\lambda_{2}}\right\rangle$ usam a base de spin $\{|+\rangle,|-\rangle\}$. Escreverei este operador também nesta base usando a matriz $\mathcal{U}$ (equação 3.2.2) - a mesma matriz que diagonaliza o acoplamento spin-órbita na seção 3.2 ,

$$
\mathcal{U} \boldsymbol{\sigma} \cdot \hat{z} \times \boldsymbol{\pi} \mathcal{U}^{-1}=\left(\begin{array}{cc}
\pi_{x} \cos (\theta)+\pi_{y} \sin (\theta) & i \pi_{x} \sin (\theta)-i \pi_{y} \cos (\theta) \\
-\pi_{x} \sin (\theta)+i \pi_{y} \cos (\theta) & -\pi_{x} \cos (\theta)-\pi_{y} \sin (\theta)
\end{array}\right)
$$


em que

$$
\cos (\theta)=\frac{k_{x}}{k_{\| 1}} \quad \text { e } \quad \sin (\theta)=\frac{k_{y}}{k_{\|}}
$$

Com isso não precisamos voltar à antiga base de spin. A ação deste operador nos vetores $| \pm\rangle$ pode ser calculada, usando a equação 5.2.22, como

$$
\boldsymbol{\sigma} \cdot \hat{z} \times \boldsymbol{\pi}| \pm\rangle= \pm \boldsymbol{\pi}_{\|} \cdot \boldsymbol{k}_{\| 1}| \pm\rangle \pm \hat{z} \cdot \boldsymbol{k}_{\| 1} \times \boldsymbol{\pi}_{\|}|\mp\rangle
$$

Desta forma, os elementos de matriz podem ser facilmente calculados

$$
\begin{aligned}
& \left\langle\boldsymbol{k}_{\| \varrho_{1}}^{\varrho_{2}}\left|\eta\left(\pi_{x} \sigma_{y}-\pi_{y} \sigma_{x}\right)\right| \boldsymbol{k}_{\| \lambda_{1}}^{\lambda_{2}}\right\rangle=\left[B_{\varrho_{1} \varrho_{2}}^{\boldsymbol{k}_{\|}} B_{\lambda_{1} \lambda_{2}}^{\boldsymbol{k}_{\|}}\left\langle u_{0}|\eta| u_{0}\right\rangle+\right. \\
& \left.\left\langle u_{1}|\eta| u_{1}\right\rangle+\left\langle u_{0}|\eta| u_{1}\right\rangle\left(B_{\varrho_{1} \varrho_{2}}^{\boldsymbol{k}_{\|}}+B_{\lambda_{1} \lambda_{2}}^{\boldsymbol{k}_{\|}}\right)\right]\left\langle\varrho_{1}\left|\pi_{x} \sigma_{y}-\pi_{y} \sigma_{x}\right| \lambda_{1}\right\rangle .
\end{aligned}
$$

Usando as definições dos acoplamentos spin-órbita 3.3.4 e a equação 5.2.24,

$$
\begin{gathered}
\left\langle\boldsymbol{k}_{\| \varrho_{1}}^{\varrho_{2}}\left|\eta\left(\pi_{x} \sigma_{y}-\pi_{y} \sigma_{x}\right)\right| \boldsymbol{k}_{\| \lambda_{1}}^{\lambda_{2}}\right\rangle= \\
\quad\left[B_{\varrho_{1} \varrho_{2}}^{\boldsymbol{k}_{\|}} B_{\lambda_{1} \lambda_{2}}^{\boldsymbol{k}_{\|}} \eta_{00}+\eta_{11}+\eta_{01}\left(B_{\varrho_{1} \varrho_{2}}^{\boldsymbol{k}_{\|}}+B_{\lambda_{1} \lambda_{2}}^{\boldsymbol{k}_{\|}}\right)\right] \times \\
\lambda_{1}\left(\boldsymbol{\pi}_{\|} \cdot \boldsymbol{k}_{\|}\left\langle\varrho_{1} \mid \lambda_{1}\right\rangle+\hat{z} \cdot \boldsymbol{k}_{\|} \times \boldsymbol{\pi}_{\|}\left\langle\varrho_{1} \mid-\lambda_{1}\right\rangle\right) .
\end{gathered}
$$

O termo $\left\langle\varrho_{1} \mid-\lambda_{1}\right\rangle$ representa a possibilidade de inversão de spin. Além disso, ficam explícitas as dependências nos acoplamentos spin-órbita inter e intrasub-bandas. No caso simétrico, $\eta_{j j}=0$ e, portanto, só existe troca de spin opticamente induzido por causa do acoplamento intersub-bandas.

Antes de analisar mais aprofundadamente este resultado, vamos voltar a uma discussão que levantamos na seção 5.2.1. Modelos de uma banda não podem prever transições intersubbandas dependentes de $\pi_{\|}$nem de spin-flips. O acoplamento spin-órbita é obra exclusiva da banda de valência, e assim também é o acoplamento assistido por spin. Note que este acoplamento não só depende apenas de $\boldsymbol{\pi}_{\|}$, como também induz spin-flip.

Grafiquei o quadrado do módulo para uma região do espaço recíproco na figura 5.2(a) para o caso simétrico de $\operatorname{InSb} / \operatorname{AlInSb} \operatorname{com} \varrho_{1}=-\lambda_{1}$ e $\boldsymbol{\pi}=(1 / \sqrt{2}, 1 / \sqrt{2})$. Há uma linha em que $\left|\left\langle\boldsymbol{k}_{\| \varrho_{1}}^{\varrho_{2}}\left|\eta\left(\pi_{x} \sigma_{y}-\pi_{y} \sigma_{x}\right)\right| \boldsymbol{k}_{\| \lambda_{1}}^{\lambda_{2}}\right\rangle\right|^{2}$ se anula por uma razão muito simples: é a solução de

$$
\pi_{x} k_{y}=\pi_{y} k_{x}
$$

É fácil verificar que, para $\varrho_{1}=-\lambda_{1}$, a expressão 5.2 .26 se anulam nesta reta. Já na figura 5.2(b) vemos algumas curvas para diferentes caminhos na rede recíproca. A curva vermelha 


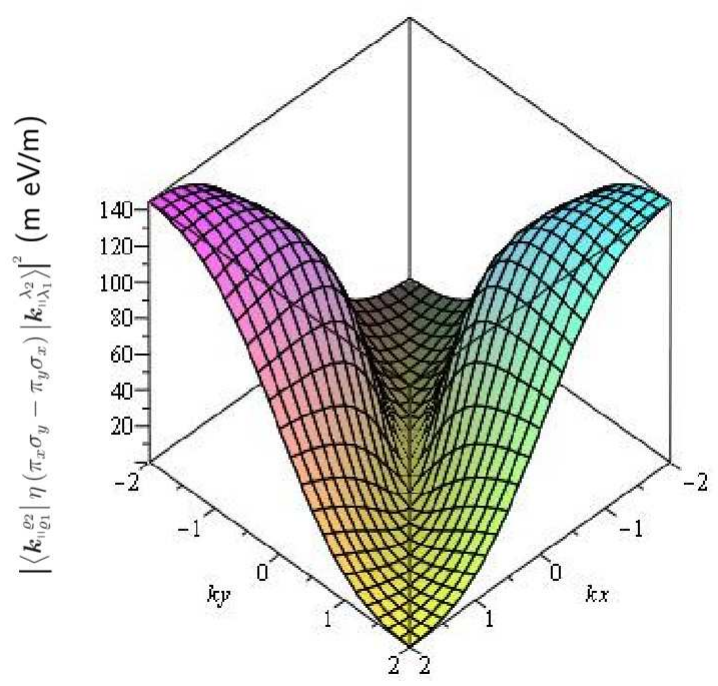

(a) Superfície do elemento de matriz

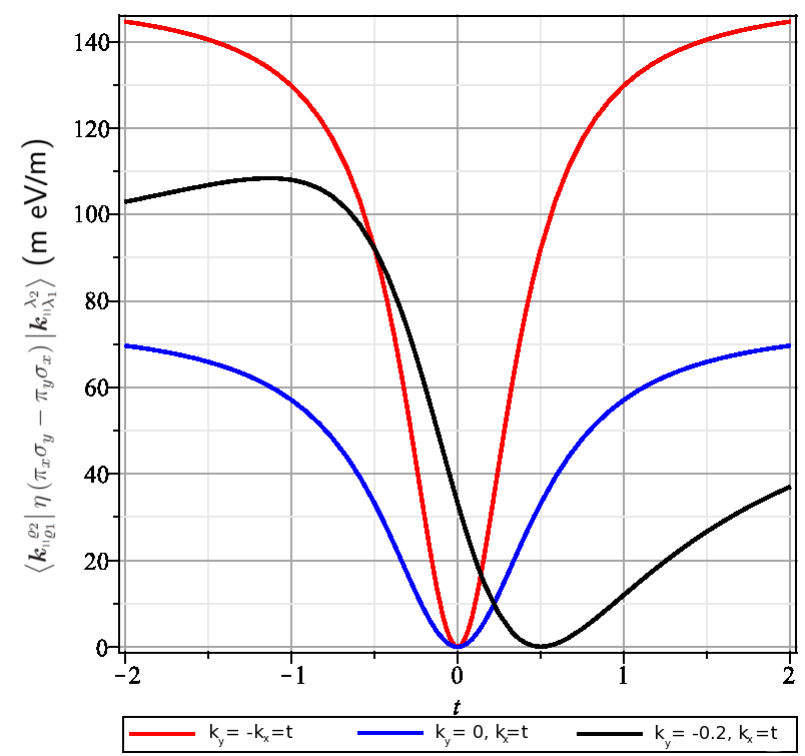

(b) Assimetria nas direções da rede recíproca

Figura 5.2 - Gráficos do quadrado do módulo do elemento de matriz do acoplamento assistido por spin para uma região da rede recíproca. Nesta transição, $\lambda_{j}=-\varrho_{j}$, ou seja, há troca de spin e de sub-banda. Podemos ver claramente uma região em que o elemento se anula. Esta região corresponde à reta $\pi_{x} k_{y}=\pi_{y} k_{y}$. Estes resultados são para $\operatorname{In} S b / A l_{0.2} \operatorname{In} n_{0.8} S b$ a $0.3 \mathrm{~K}$. Mantive o potencial químico fixo em todas estas simulações $(100 \mathrm{meV})$, com a densidade de doadores de $3 \times 10^{18} \mathrm{~cm}^{-2}$. A região dos doadores tem largura fixa de $4 \mathrm{~nm}$.

mostra o caminho em que o $\left|\left\langle\boldsymbol{k}_{\| \varrho_{1}}^{\varrho_{2}}\left|\eta\left(\pi_{x} \sigma_{y}-\pi_{y} \sigma_{x}\right)\right| \boldsymbol{k}_{\| \lambda_{1}}^{\lambda_{2}}\right\rangle\right|^{2}$ é máximo, isto é,

$$
\pi_{x} k_{y}=-\pi_{y} k_{x}
$$

Como usamos $\pi_{x}=\pi_{y}$, então o caminho que minimiza e maximiza o elemento de matriz são as diagonais, i.e., os caminhos [110] e [110], respectivamente. As curvas preta e azul da figura 5.2(b) representam casos diferentes, em que $k_{y}$ fixa-se em $0.2 \mathrm{~nm}$ e $0 \mathrm{~nm}$, respectivamente.

Nas figuras 5.3(a) e 5.3(b) fiz exatamente o mesmo, mas usando $\varrho_{1}=\lambda_{1}$, ou seja, sem troca de spin. Continua existindo uma linha em que o elemento é máximo, $\pi_{x} k_{x}=-\pi_{y} k_{x}$, e uma em que o elemento é nulo, $\pi_{x} k_{x}=\pi_{y} k_{x}$.

Outro fato interessante com este acoplamento é que, no caso simétrico, a taxa é limitada na zona de Brillouin, i.e., existe uma saturação quando há spin flip e mudança de sub-banda Parametrizarei por $(t, \theta)^{\S}$ o caminho $\boldsymbol{k}_{\Perp}(t, \theta):=(t \cos (\theta), t \sin (\theta))$ na zona de Brillouin. Então,

\footnotetext{
¥Usando a notação que adotamos desde o começo do capítulo, fixamos $\varrho_{1}=-\lambda_{1}$ e $\varrho_{2}=-\lambda_{2}$.

$\S$ Parametrização com coordenadas polares.
} 


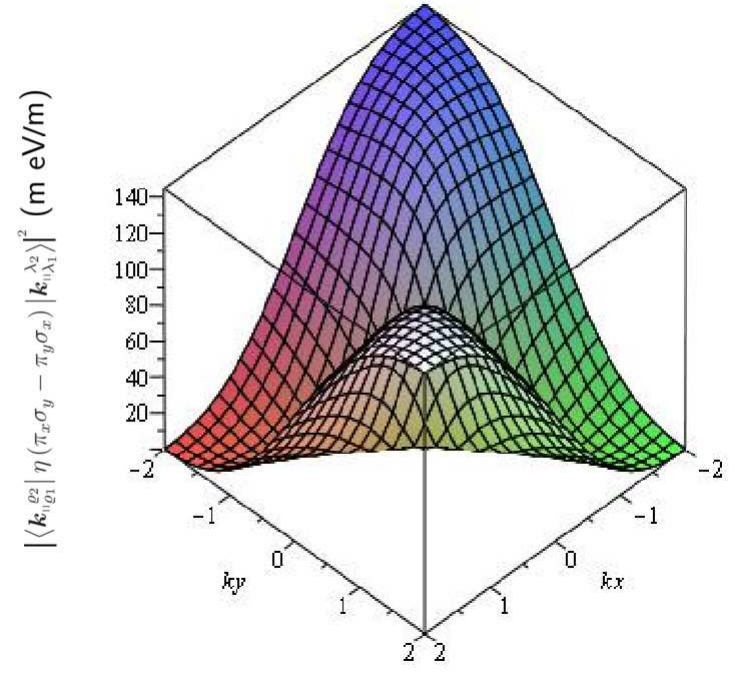

(a) Superfície do elemento de matriz

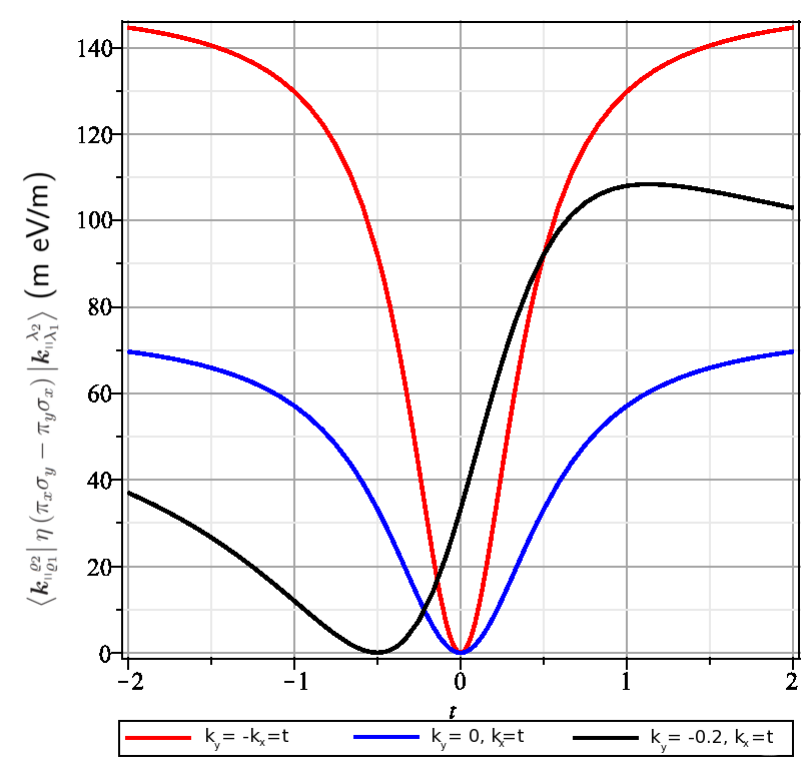

(b) Assimetria nas direções da rede recíproca

Figura 5.3 - Mesmo que na figura 5.2(a), mas com $\pi_{x}=-\pi_{y}$. Note que a linha de transição nula agora tem seu ângulo modificado por $\pi / 2$. Também mostro o análogo da figura 5.2(b), em que podemos ver caminhos específicos da zona de Brillouin. Estes resultados são para $\mathrm{InSb} / \mathrm{Al}_{0.2} \mathrm{In}_{0.8} S b$ a $0.3 \mathrm{~K}$. Mantive o potencial químico fixo em todas estas simulações $(100 \mathrm{meV})$, com a densidade de doadores de $3 \times 10^{18} \mathrm{~cm}^{-2}$. A região dos doadores tem largura fixa de $4 \mathrm{~nm}$.

calculei exatamente a distribuição dos valores assintóticos em função de $\theta$ :

$$
\lim _{t \rightarrow \infty}\left\langle\boldsymbol{k}_{\|}(t, \theta)_{ \pm \lambda_{1}}^{-\lambda_{2}}\left|\eta\left(\pi_{x} \sigma_{y}-\pi_{y} \sigma_{x}\right)\right| \boldsymbol{k}_{\|}(t, \theta)_{\lambda_{1}}^{\lambda_{2}}\right\rangle=-\frac{2 \widetilde{\epsilon}_{-}}{5}\left[\cos (\theta) \pi_{x}+\lambda_{1} \varrho_{1} \sin (\theta) \pi_{y}\right],
$$

ou seja, depende apenas da diferença dos espaçamentos dos níveis das sub-bandas ${ }^{\pi}$. Nos exemplos acima, $\pi_{j}=1 / \sqrt{2}$ para $j=x, y, \varrho_{1}=-\lambda_{1}$ e a linha em que o elemento de matriz se anulava equivale a $\theta=\pi / 4$. Calculando, é fácil verificar que este é um zero da distribuição. Equivalentemente, podemos calcular o valor máximo da saturação:

$$
\theta=\frac{3 \pi}{4} \rightarrow \lim _{t \rightarrow \infty}\left\langle\boldsymbol{k}_{\|}(t, \theta)_{-\lambda_{1}}^{-\lambda_{2}}\left|\eta\left(\pi_{x} \sigma_{y}-\pi_{y} \sigma_{x}\right)\right| \boldsymbol{k}_{\|}(t, \theta)_{\lambda_{1}}^{\lambda_{2}}\right\rangle=0
$$

Também podemos ver que se $\varrho_{1}=\lambda_{1}$, a distribuição tem um máximo e um zero, mas as raízes mudam. Em geral, essas retas, em que os elementos de matriz se anulam, são dadas por

$$
\operatorname{cotan}(\theta)=-\lambda_{1} \varrho_{1} \frac{\pi_{y}}{\pi_{x}}
$$

\footnotetext{
TTalvez compense relembrar a definição dada na equação 3.5.4, a saber, $2 \widetilde{\epsilon}_{-}=\widetilde{\epsilon}_{1}-\widetilde{\epsilon}_{0}$.
} 


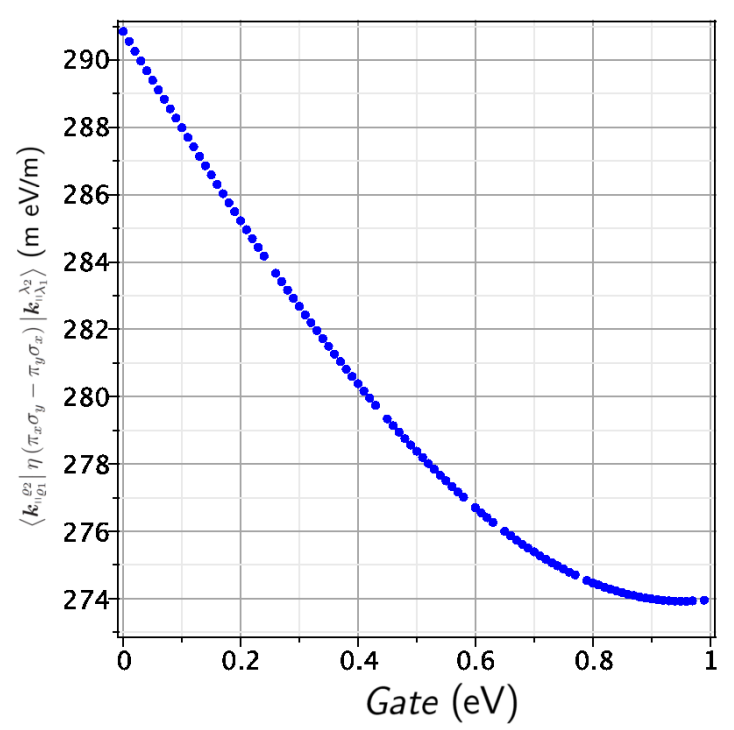

(a) Variação com gates

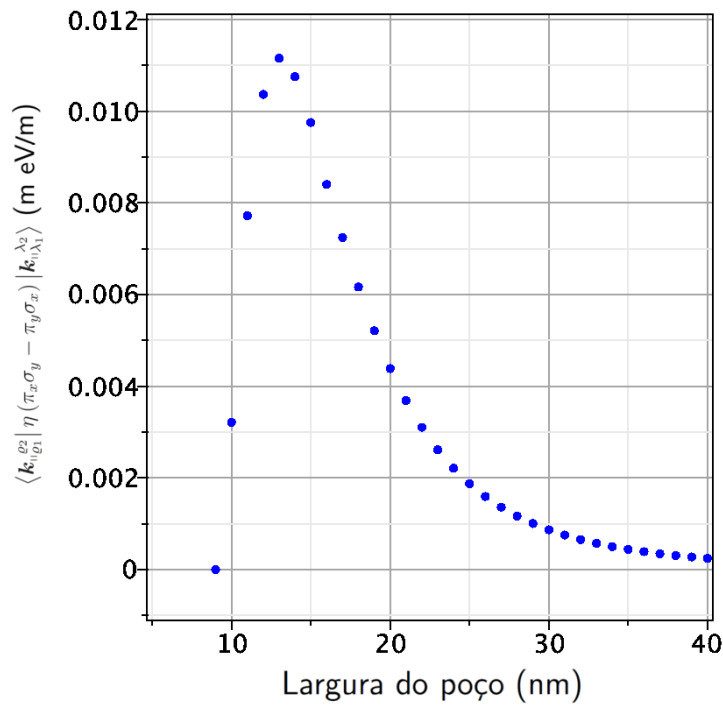

(b) Variação com a largura

Figura 5.4 - Influência assintótica da variação de gates e largura do poço quântico nas taxas do acoplamento assistido por spin. Vemos que o máximo ocorre no caso simétrico (gates nulos). Estes resultados são para $\operatorname{InSb} / \mathrm{Al}_{0.2} \mathrm{In} n_{0.8} S b$ a $0.3 \mathrm{~K}$. Mantive o potencial químico fixo em todas estas simulações $(100 \mathrm{meV})$, com a densidade de doadores de $3 \times 10^{18} \mathrm{~cm}^{-2}$. A região dos doadores tem largura fixa de $4 \mathrm{~nm}$.

Já para as retas em que os elementos são máximos,

$$
\tan (\theta)=\lambda_{1} \varrho_{1} \frac{\pi_{y}}{\pi_{x}}
$$

No caso assimétrico, podemos mostrar que os elementos de matriz sempre divergem para $t \rightarrow \infty$, independentemente da escolha de $\theta$. Ainda assim, há a observação de linhas no espaço recíproco de máximos e mínimos.

Esses comportamentos assintóticos não são tão intuitivos, em geral as expressões divergem para grandes $k_{\|}$. Esta divergência, já observada no acoplamento $\boldsymbol{P} \cdot \boldsymbol{A}$, também ocorre nas transições sem troca de sub-banda e no acoplamento estranho. Portanto, a observação deste perfil saturado seria uma assinatura não apenas do acoplamento assistido por spin, como também de transições intersub-bandas.

\subsubsection{Aplicação de gates e largura do poço}

A aplicação de gates quebra a simetria de inversão do potencial e alguns termos adicionais são invocados nos elementos de matriz. A figura 5.4(a) mostra como a taxa varia, assintoticamente, com o campos elétricos externos (gates). Mostro apenas o comportamento 
assintotico porque, quando há quebra de simetria, então não há mais a saturação que comentamos anteriormente. Podemos notar imediatamente que a taxa é máxima quando o gate é nulo (caso simétrico). A varição é bem razoável assintoticamente, e por isso há a possibilidade do controle destas taxas via campos externos.

Como o caso simétrico apresenta a maior taxa, anularemos os gates para o próximo experimento. Variei a largura do poço e verifiquei uma dependência interessante nos elementos de matriz, mostrada na figura 5.4(b).

Existe um valor da largura do poço, dada por $13 \mathrm{~nm}$, que maximiza a transição por um fator de $10^{2}$. Para poços menores que $9 \mathrm{~nm}$, os elétrons não são confinados. Infelizmente, como $\eta_{01}$ e $\widetilde{\epsilon}_{-}$dependem da largura do poço de alguma forma não analítica, não podemos explicar analiticamente estes resultados. 


\subsection{Revisão}

As frequências características aceitas por poços quânticos de materiais $I I I-V$ estão na faixa do infravermelho próximo. Elementos de matriz óptica:

1. Acoplamento $\boldsymbol{P} \cdot \boldsymbol{A}$ :

$$
\begin{array}{r}
\left\langle\boldsymbol{k}_{\| \varrho_{1}}^{\varrho_{2}} ; 0\left|\frac{\hbar}{2 M} \boldsymbol{k} \cdot \boldsymbol{A}\right| \boldsymbol{k}_{\| \lambda_{1}}^{\lambda_{2}} ; 1_{\boldsymbol{q} \boldsymbol{\pi}}\right\rangle=\frac{\mathcal{C}}{\sqrt{\omega_{q}}} \pi_{z} \delta_{\varrho_{1}, \lambda_{1}} A_{\lambda_{1} \lambda_{2}}^{\boldsymbol{k}_{\|}} A_{\varrho_{1} \varrho_{2}}^{\boldsymbol{k}_{\|}}\left(B_{\varrho_{1} \varrho_{2}}^{\boldsymbol{k}_{\|}} B_{\lambda_{1} \lambda_{2}}^{\boldsymbol{k}_{\|}}\left\langle u_{0}\left|k_{z}\right| u_{0}\right\rangle+\right. \\
\left.B_{\varrho_{1} \varrho_{2}}^{\boldsymbol{k}_{\boldsymbol{N}_{1}}}\left\langle u_{0}\left|k_{z}\right| u_{1}\right\rangle+B_{\lambda_{1} \lambda_{2}}^{\boldsymbol{k}_{\|}}\left\langle u_{1}\left|k_{z}\right| u_{0}\right\rangle+\left\langle u_{1}\left|k_{z}\right| u_{1}\right\rangle\right) .
\end{array}
$$

2. Acoplamento radiação-momento-campo elétrico:

$$
\begin{aligned}
& \left\langle\boldsymbol{k}_{\| \varrho_{1}}^{\varrho_{2}} ; 0\left|\frac{\alpha_{M} e}{\hbar c} \boldsymbol{k} h_{w} \cdot \boldsymbol{A}\right| \boldsymbol{k}_{\| \lambda_{1}}^{\lambda_{2}} ; 1_{\boldsymbol{q} \boldsymbol{\pi}}\right\rangle=\frac{e \alpha_{M}}{\hbar c} \sqrt{\frac{2 \pi \hbar c^{3}}{V}} \pi_{z} \\
& A_{\lambda_{1} \lambda_{2}}^{\boldsymbol{k}_{\|}} A_{\lambda_{1} \lambda_{2}}^{\boldsymbol{k}_{\|}}\left[B_{\varrho_{1} \varrho_{2}}^{\boldsymbol{k}_{\lambda_{1}}} B_{\lambda_{1} \lambda_{2}}^{\boldsymbol{k}_{\|}} u_{0}^{2}\left(a_{0}\right)+u_{1}^{2}\left(a_{0}\right)+u_{0}\left(a_{0}\right) u_{1}\left(a_{0}\right)\left(B_{\varrho_{1} \varrho_{2}}^{\boldsymbol{k}_{\boldsymbol{k}^{\prime}}}+B_{\lambda_{1} \lambda_{2}}^{\boldsymbol{k}_{\|}}\right)-\right. \\
& \left.B_{\varrho_{1} \varrho_{2}}^{\boldsymbol{k}_{\|}} B_{\lambda_{1} \lambda_{2}}^{\boldsymbol{k}_{\|}} u_{0}^{2}\left(-a_{0}\right)-u_{1}^{2}\left(-a_{0}\right)-u_{0}\left(-a_{0}\right) u_{1}\left(-a_{0}\right)\left(B_{\varrho_{1} \varrho_{2}}^{\boldsymbol{k}_{\|}}+B_{\lambda_{1} \lambda_{2}}^{\boldsymbol{k}_{\|}}\right)\right] .
\end{aligned}
$$

3. Acoplamento assistido por spin:

$$
\begin{aligned}
\left\langle\boldsymbol{k}_{\| !}^{\varrho_{2}} ; 0\left|-\frac{i e}{\hbar c} \boldsymbol{\sigma} \cdot \boldsymbol{k} \tilde{\eta} \times \boldsymbol{A}\right| \boldsymbol{k}_{\| \lambda_{1}}^{\lambda_{2}} ; 1_{\boldsymbol{q} \boldsymbol{\pi}}\right\rangle= & {\left[B_{\varrho_{1} \varrho_{2}}^{\boldsymbol{k}_{\|}} B_{\lambda_{1} \lambda_{2}}^{\boldsymbol{k}_{\|}} \eta_{00}+\eta_{11}+\eta_{01}\left(B_{\varrho_{1} \varrho_{2}}^{\boldsymbol{k}_{\|}}+B_{\lambda_{1} \lambda_{2}}^{\boldsymbol{k}_{\|}}\right)\right] \times } \\
& \lambda_{1}\left(\boldsymbol{\pi}_{\|} \cdot \boldsymbol{k}_{\|}\left\langle\varrho_{1} \mid \lambda_{1}\right\rangle+\hat{z} \cdot \boldsymbol{k}_{\|} \times \boldsymbol{\pi}_{\|}\left\langle\varrho_{1} \mid-\lambda_{1}\right\rangle\right) .
\end{aligned}
$$

Usamos as definições

$$
\begin{aligned}
\alpha_{D} & =-\frac{\mathcal{P}^{2}}{6}\left[\frac{2}{E_{g}^{2}}+\frac{1}{\left(E_{g}+\Delta_{g}\right)^{2}}\right], \\
\alpha_{M} & =-\frac{\mathcal{P}^{2}}{3}\left[\gamma_{2}^{\prime}+\gamma_{2} \delta_{6}\right] .
\end{aligned}
$$

O acoplamento assistido por spin é o único que fornece a possbilidade de (i) spin flip; (ii) transição óptica induzida por polarização no plano. Observamos também uma saturação em $k_{11}$, e a distribuição dos valores assintóticos segue

$$
\lim _{t \rightarrow \infty}\left\langle\boldsymbol{k}_{\|}(t, \theta)_{ \pm \lambda_{1}}^{-\lambda_{2}}\left|\eta\left(\pi_{x} \sigma_{y}-\pi_{y} \sigma_{x}\right)\right| \boldsymbol{k}_{\Perp}(t, \theta)_{\lambda_{1}}^{\lambda_{2}}\right\rangle=-\frac{2 \widetilde{\epsilon}_{-}}{5}\left[\cos (\theta) \pi_{x}+\lambda_{1} \varrho_{1} \sin (\theta) \pi_{y}\right] .
$$


As soluções estão descritas em termos de

$$
\begin{gathered}
\left|\lambda_{1} \lambda_{2}\right\rangle=A_{\lambda_{1} \lambda_{2}}^{\boldsymbol{k}_{\|}}\left(A_{\lambda_{1} \lambda_{2}}^{\boldsymbol{k}_{\|}}\left|u_{0}\right\rangle+\left|u_{1}\right\rangle\right) \otimes\left|\lambda_{1}\right\rangle . \\
B_{\lambda_{1} \lambda_{2}}^{\boldsymbol{k}_{\|}}=\frac{\eta_{01}\left(k-k_{0}\right)}{\lambda_{2} \Omega_{\lambda_{1}}(k)+\left(\widetilde{\epsilon}_{-}+\lambda_{1} \alpha_{-}\right)} \text {e } \quad A_{\lambda_{1} \lambda_{2}}^{\boldsymbol{k}_{\|}}=\left\{1+\left(B_{\lambda_{1} \lambda_{2}}^{\boldsymbol{k}_{\|}}\right)^{2}\right\}^{-1 / 2} . \\
\alpha_{ \pm}=\frac{\eta_{11 \pm \eta_{00}}}{2}, k_{0}=\frac{H_{d e}^{01}+H_{M E C}^{01}}{\eta}, \widetilde{\epsilon}_{j}=\epsilon_{j}+H_{d e}^{j j}+H_{M E C}^{j j}, \widetilde{\epsilon}_{ \pm}=\frac{\widetilde{\epsilon}_{1} \pm \widetilde{\epsilon}_{0}}{2} \\
\Omega_{\lambda_{1}}^{2}(k)=\left(2 \widetilde{\epsilon}_{-}+\lambda_{1} \alpha_{-} k_{\|}\right)^{2}+\eta_{01}^{2}\left(k_{\|}-k_{0}\right)^{2} .
\end{gathered}
$$

Além disso, utilizei as seguintes definições:

$$
\begin{gathered}
\tilde{\eta}=\gamma_{H} V+\gamma_{e} h_{w} \quad \text { e } \eta=\gamma_{H} \frac{d V}{d z}+\gamma_{e} \frac{d h_{w}}{d z} . \\
\eta_{i j}=\left\langle u_{i}|\eta| u_{j}\right\rangle \\
\eta_{H}=\frac{1}{E_{g}^{2}}-\frac{1}{\left(E_{g}+\Delta_{g}\right)^{2}}, \\
\eta_{e}=\frac{\delta_{8}}{E_{g}^{2}}-\frac{\delta_{7}}{\left(E_{g}+\Delta_{g}\right)^{2}} .
\end{gathered}
$$

Termo de Darwin estrutural:

$H_{d e}^{n m}=-\delta_{6}\left[\phi_{n}\left(a_{0}\right) \frac{d \phi_{m}}{d z}\left(a_{0}\right)+\phi_{m}\left(a_{0}\right) \frac{d \phi_{n}}{d z}\left(a_{0}\right)-\phi_{n}\left(-a_{0}\right) \frac{d \phi_{m}}{d z}\left(-a_{0}\right)-\phi_{m}\left(-a_{0}\right) \frac{d \phi_{n}}{d z}\left(-a_{0}\right)\right]$.

Acoplamento momento-campo elétrico:

$$
H_{M E C}^{n m}=-\alpha_{M} \delta_{\mu, \nu}\left[u_{n}\left(a_{0}\right) \frac{d u_{m}}{d z}\left(a_{0}\right)-u_{n}\left(-a_{0}\right) \frac{d u_{m}}{d z}\left(-a_{0}\right)\right] .
$$




\section{CAPÍTULO 6}

\section{Conclusões do Trabalho}

To find your fame and fortune trhough the valley you must walk

You will face your inner deamons

Now go, my son, and rock!

Dio, em Tenacious D - Kickapoo, 2007.

A partir da equação de Dirac, é possível deduzir, por meio de expansões em potências de $1 / c$, equações que lembram a equação de Schrödinger por terem duas componentes (spin). Este método é conhecido como Folding Down, por eliminar duas (das quatro) componentes da equação de Dirac. Em primeira ordem, temos a equação de Pauli; em segunda ordem, vemos termos inesperados (acoplamento spin-órbita, termo de Darwin e correção da massa). Ao longo deste trabalho, principalmente no capítulo 2, realizei um procedimento em analogia ao folding down, em que eliminamos das contas a necessidade das funções de onda das bandas de valência. Além de a correção relativística da massa não ter aparecido na hamiltoniana efetiva, surgiu um termo sem análogo, que nomei de o acoplamento momento-campo elétrico. Se realizarmos o folding down na equação de Dirac considerando potenciais diferentes para as partículas e as antipartículas, obteremos em segunda ordem de $1 / c^{2}$ um termo bem parecido com este acoplamento momento campo-elétrico (realizo este cálculo no apêndice B).

O ponto central do Folding Down está na redução do espaço vetorial. Durante o Folding Down, redefini a função de onda eletrônica para trabalharmos com um spinor de apenas duas componentes, mas que contivesse a informação de todas as oito componentes no que se refere à distribuição de probabilidades. Podemos dizer que este novo spinor dá à banda de condução uma interpretação probabilística - ou dá caráter de função de onda, muito embora este novo spinor não seja a função de onda orignal: trata-se de um novo objeto cujo quadrado do módulo é sempre igual à densidade de probabilidade do spinor original de oito componentes. 
A praticidade Folding Down que desenvolvi deve-se ao desenvolvimento das identidades algébricas demonstradas na seção 2.5. Com estas identidades, somos capazes de escrever em séries de potências de $1 / E_{g}$ (gap do material central) a equação efetiva para a banda de condução. Mostramos explicitamente a equação efetiva resultante até segunda ordem em $1 / E_{g}$. Esta equação efetiva vale para poços, fios e pontos quânticos, dada a arbitrariedade que mantemos nos potenciais $E_{6}, E_{7}$ e $E_{8}$ ao longo dos cálculos. Este propcedimento pode ser estendido a ordens mais altas de $1 / E_{g}$ trivialmente. Além disso, o procedimento não se restringe ao tipo de poço quântico com que trabalhamos, podendo ser aplicado a poços de tipo $I, I I$ e $I I I$.

Muitos modelos incluem o acoplamento elétron-fóton na hamiltoniana de Kane $(8 \times 8)$, mesmo que o interesse seja apenas a banda de condução. Assim como somos capazes de fazer eletrodinâmica na aproximação relativística da equação de Dirac, incluí no capítulo 4 campos eletromagnéticos na equação efetiva para a banda de condução. Desta forma, não precisamos carregar as seis componentes da banda de valência ao calcular os elementos de matriz que fornecem taxas de transições ópticas. Para tal resultado, usamos o fato de o poço ser muito estreito se comparado ao comprimento de onda admitido para excitações ressonantes (as frequências admitidas para transições ópticas estão na faixa do infra-vermelho próximo seção 4.3). Assim, o potencial vetor pode ser encarado como uma função (campo vetorial, neste caso) que varia lentamente, e adicionado à aproximação das funções envelope de forma análoga ao potencial estrutural $(\phi(\boldsymbol{r})$ na seção 2.3). Deduzi expressões analíticas para os acoplamentos elétron-fóton até segunda ordem de $1 / E_{g}$, e possíveis extensões desta ordem podem ser implementadas de forma quase trivial. Mostrei que estes acoplamentos seguem em estrita analogia com os acoplamentos elétron-fóton gerados pela equação de Dirac.

Aplicamos nossa hamiltoniana efetiva resultante para o caso em que o poço quântico comporta dois estados ligados. Estes estados desdobram-se em quatro por causa do spin. Abordamos esta solução de forma autoconsistente em ordem zero de $1 / E_{g}$. Todos os termos de segunda ordem em $1 / E_{g}$ foram tratados utilizando como base as soluções autoconsistentes de ordem zero. Mostrei como diagonalizar estes termos de segunda ordem usando uma abordagem similar à feita na dissertação de Calsaverini ${ }^{1}$ e apresentei as soluções completas e suas relações de dispersão. Podemos trivialmente incluir barreiras ou mais poços (veja a seção 3.1.3). Verifiquei o desdobramento dos níveis causado pelo acoplamento intrasub-bandas na seção 3.5.2. Por fim, reescrevendo a hamiltoniana efetiva numa forma que lembra a interação Zeeman, mostrei na seção 3.8 uma pequena generalização para o conceito de campos magnéticos internos quando há mais de uma sub-banda. 
Neste caso em que temos duas sub-bandas, mostramos em detalhes como são os elementos de matriz das interações elétron-fóton em ordem mais baixa em perturbação dependente do tempo. Verifiquei que o único acoplamento elétron-fóton que induz transições ópticas com polarização em plano é o acoplamento assistido por spin. Este acoplamento também é o único que fornece a possibilidade de transições com spin flip. Também mostrei algumas peculiaridades do acoplamento assistido por spin, como a saturação nas taxas de transições intersub-bandas ou como as retas na zona de Brillouin em que estas taxas se anulam por completo.

Com este estudos, espero abrir possibilidades para estudar em maiores detalhes transições ópticas e expandir nossos resultados para outras estruturas. Há alguns projetos para um futuro quase imediato:

1. adicionar à autoconsistência os termos de segunda ordem em $1 / E_{g}$, o que pode ser implementado resolvendo a equação diferencial 3.1.1, desacoplada pela nova base de spin introduzida na seção 3.2;

2. estimar o termo de Darwin e o acoplamento momento-campo elétrico em diversas outras estruturas

3. analisar as taxas de transições para uma variedade de poços quânticos, incluindo poços formados por GaAs (Arseneto de Gálio), InAs (Arseneto de Índio), GaSb (Antimoneto de Gálio), etc;

4. investigar as aproximações de dipolo magnético e dipolo elétrico - possível surgimento de novas regras de seleção de spin;

5. realizar o Folding Down para estruturas de rede Wurtzita e obter uma hamiltoniana efetiva na presença de campos, determinando assim os acoplamentos elétron-fóton. 


\section{REFERÊNCIAS}

1 CALSAVERINI, R. S. P. A. Acoplamento spin-órbita inter-subbanda em heteroestruturas semicondutoras. 2007. 97 p. Dissertação (Mestrado em Física) - Instituto de Física de São Carlos - USP, 2007.

2 HACHIYA, M. A. O. Efeito Hall de spin em poços quânticos com acoplamento spin-órbita inter-subbanda. 2009. 93 p. Dissertação (Mestrado em Física) - Instituto de Física de São Carlos - USP, 2009.

3 BERNARDES, E.; SCHLIEMANN, J.; LEE, M.; EGUES, J. C.; LOSS, D. Spin-orbit interaction in symmetric wells with two subbands. Physical Review Letters, Woodbury, v. 99, n. 7, p. 076603-1-076603-4, 2007.

4 CALSAVERINI, R. S.; BERNARDES, E.; EGUES, J. C.; LOSS, D. Intersubband-induced spin-orbit interaction in quantum wells. Physical Review B, v. 78, n. 15, p. 155313-1155313-16, 2008.

5 LEE, M.; HACHIYA, M. O.; BERNARDES, E.; EGUES, J. C.; LOSS, D. Spin Hall effect due to intersubband-induced spin-orbit interaction in symmetric quantum wells. Physical Review B, v. 80, n. 15, p. 155314-1-155314-7, 2009.

6 SWART, J. W. Semiconductores: fundamentos, técnicas e aplicações. Campinas: Unicamp, 2008.

7 COHEN-TANnOUDJI, C.; DIU, B. L. F. Quantum mechanics. New York: John Wiley, 1977.

8 LOSS, D.; DIVINCENZO, D. P. Quantum computation with quantum dots. Physical Review A, Woodbury, v. 57, n. 1, p. 120-126, 1998.

9 MOKKAPATI, S.; JAGADISH, C. III-V compound SC for optoelectronic devices. Materials Today, v. 12, n. 4, p. 22-32, 2009. 
10 ANIKEEVA, P. O.; HALPERT, J. E.; BAWENDI, M. G.; BULOVI, V. Quantum dot light-emitting devices with electroluminescence tunable over the entire visible spectrum. Nano Letters, v. 9, n. 7, p. 2532-2536, 2009.

11 DE FARIA JUNIOR, P.; SIPAHI, G. M. Polytypic nanowhiskers: electronic properties in the vicinity of the band edges. Disponível em: <http://arxiv.org/abs/1012.0227v1>. Acesso em: Dezembro de 2010.

12 LIU, R.-B.; YAO, W.; SHAM, L. J. Quantum computing by optical control of electron spins. Advances in Physics, London, v. 59, n. 5, p. 703-802, 2010.

13 RECHER, P.; TRAUZETTEL, B. Quantum dots and spin qubits in graphene. Nanotechnology, v. 21, n. 30, p. 302001-1-302001-13, 2010.

14 MARK, J.; SILSBEE, R. H. Interfacial charge-spin coupling: injection and detection of spin magnetization in metals. Physical Review Letters, Woodbury, v. 55, n. 17, p. 1790-1793, 1985.

15 BAIBICH, M. N.; BROTO, J. M.; FERT, A.; DAU, F. N. V.; PETROFF, F.; ETIENNE, P.; CREUZET, G.; FRIEDERICH, A.; CHAZELAS, J. Giant magnetoresistance of (001) $\mathrm{Fe} /(001) \mathrm{Cr}$ magnetic superlattices. Physical Review Letters, Woodbury, v. 61, n. 21, p. $2472-2475,1988$.

16 BINASCH, G.; GRÜNBERG, P.; SAURENBACH, F.; ZINN, W. Enhanced magnetoresistance in layered magnetic structures with antiferromagnetic interlayer exchange. Physical Review B, v. 39, n. 7, p. 4828-4830, 1989.

17 FABIAN, J.; MATOS-ABIAGUE, A.; ERTLER, C.; STANO, P.; ŽUTIĆ, I. Semiconductors spintronics. Acta Physica Slovaca, v. 57, n. 4-5, p. 1-907, 2007.

18 DE ANDRADA E SILVA; G. C. LA ROCCA; F. BASSANI, E. A. Spin-orbit splitting of electronic states in semiconductor asymmetric quantum wells. Physical Review B, v. 55, n. 24, p. 16293-16299, 1997.

19 DE ANDRADA E SILVA, E. A. Conduction-subband anisotropic spin splitting in III-V semiconductor heterojunctions. Physical Review B, v. 46, n. 3, p. 1921-1924, 1992.

20 DRESSELHAUS, G. Spin-orbit coupling effects in zinc blende structures. Physical Review, v. 100, n. 2 , p. $580-586,1955$.

21 DRESSELHAUS, G.; KIP, A. F.; KITTEL, C. Cyclotron resonance of electrons and holes in silicon and germanium crystals. Physical Review, v. 98, n. 2, p. 368-384, 1955.

22 BERESTETSKII, V. P.; PITAEVSKII, L. P.; LIFSHITZ, E. M. Quantum electrodynamics. 2nd ed. New York: Pergamon Press, 1982. 
23 BJORKEN, J. D.; DRELL, S. D. Relativistic quantum mechanics. New York: McGraw-Hill, 1964.

24 BAGNATO, V. S. Introdução a física atômica e molecular. São Carlos: IFSC, 2008.

25 BOHM, A. Quantum mechanics. New York: Springer-Verlag, 1993.

26 FAZZIO, A.; WATARI, K. Introdução a teoria de grupos aplicada em moléculas e sólidos. Santa Maria: UFSM, 2009.

27 CRACKNELL, A. P.; BRADLEY, C. J. The mathematical theory of symmetry in solids. Oxford: Clarendon Press, 1972.

28 BYCHKOV, Y. A.; RASHBA, E. I. Oscillatory effects and the magnetic susceptibility of carriers in inversion layers. Journal of Physics C: solid state physics, v. 17, n. 33, p. 6039, 1984.

29 MEIER, L.; SALIS, G.; SHORUBALKO, I.; GINI, E.; SCHOEN, S.; ENSSLIN, K. Measurement of Rashba and Dresselhaus spin-orbit magnetic fields. Nature Physics, v. 3, n. 9, p. 650-654, 2007.

30 ZHANG, X. C.; SCOTT, G. D.; JIANG, H. W. NMR probing of spin excitations in the ring structure of a two-subband system. Physical Review Letters, Woodbury, v. 98, n. 24, p. 246802-1-246802-4, 2007.

31 LI, M.; ZHANG, R.; ZHANG, Z.; YAN, W.; LIU, B.; FU, D.; ZHAO, C.; XIE, Z.; XIU, X.; ZHENG, Y. Rashba spin splitting for the first two subbands in heterostructures. Superlattices and Microstructures, v. 47, n. 4, p. $522-529,2010$.

32 SIMMONDS, P. J.; HOLMES, S. N.; BEERE, H. E.; RITCHIE, D. A. Spin-orbit coupling in an $I_{0.52} G_{0.48} A s$ quantum well with two populated subbands. Journal of Applied Physics, Woodbury, v. 103, n. 12, p. 124506-1-124506-7, 2008.

33 FERREIRA, G. J.; FREIRE, H. J. P.; EGUES, J. C. Many-body effects on the $\rho_{x x}$ ringlike structures in two-subband wells. Physical Review Letters, Woodbury, v. 104, n. 6, p. 066803$1-066803-4,2010$.

34 FU, J. Y.; PENTEADO, P. H.; EGUES, J. C. Spin-orbit coupling in InAs-based Wurtzite quantum well. In: SÃO PAULO SCHOOL OF ADVANCED SCIENCE: spintronics and quantum computation, 1., 2010, São Carlos. Resumos... São Carlos: IFSC, c2010. p. 77.

35 BERNARDES, E.; CALSAVERINI, R.; EGUES, J. C.; LOSS, D. Resonance in the intersubband-induced spin-orbit interaction in double quantum wells. In: INTERNATIONAL CONFERENCE ON PHYSICS AND APPLICATIONS OF SPIN-RELATED PHENOMENA IN SEMICONDUCTORS, 5., 2008, Foz do Iguaçu. Resumos... São Carlos: IFSC, c2008. p. 99. 
36 GREINER, W. Relativistic quantum mechanics: wave mechanics. Berlin: Springer-Verlag, 1994. (Theoretical Physics, v. 3).

37 SCHLIEMANN, J.; LOSS, D.; WESTERVELT, R. M. Zitterbewegung of electrons and holes in III-V semiconductor quantum wells. Physical Review B, v. 73, n. 8, p. 085323-1085323-9, 2006.

38 SCHLIEMANN, J.; LOSS, D.; WESTERVELT, R. Zitterbewegung of electronic wave packets in III-V zinc-blende semiconductor quantum wells. Physical Review B, v. 94, n. 20, p. 206801-1-206801-4, 2005.

39 RUSIN, T. M.; ZAWADZKI, W. Transient zitterbewegung of charge carriers in monoand bilayer graphene, and carbon nanotubes. Physical Review B, v. 76, n. 19, p. 195439-1195439-7, 2007.

40 MAKSIMOVA, G. M.; DEMIKHOVSKII, V. Y.; FROLOVA, E. V. Wave packet dynamics in a monolayer graphene. Physical Review B, v. 78, n. 23, p. 235321-1-235321-7, 2008.

41 WINKLER, R. Spin-orbit coupling effects in two-dimensional electron and hole systems. Berlin: Springer, 2003. (Springer tracts in modern physics, v. 191).

42 DATTA, S.; DAS, B. Electronic analog of the electro-optic modulator. Applied Physics Letters, Woodbury, v. 56, n. 7, p. 665-667, 1990.

43 KOO, H. C.; KWON, J. H.; EOM, J.; CHANG, J.; HAN, S. H.; JOHNSON, M. Control of spin precession in a spin-injected field effect transistor. Science, Washington, v. 325, n. 5947, p. 1515-1518, 2009.

44 WU, M. W.; JIANG, J. H.; WENG, M. Q. Spin dynamics in semiconductors. Physics Reports-review section of physics letters, v. 493, n. 2-4, p. 61-236, 2010.

45 LIU, MING-HAO; CHANG, C.-R. Datta-das transistor: significance of channel direction, size dependence of source contacts, and boundary effects. Physical Review B, v. 73, n. 20, p. 205301-1-205301-5, 2006.

46 SHELYKH, I. A.; JOHNE, R.; SOLNYSHKOV, D. D.; MALPUECH, G. Optically and electrically controlled polariton spin transistor. Physical Review B, v. 82, n. 15, p. 153303$1-153303-4,2010$.

47 LIU, D.-Y.; XIA, J.-B. Rashba electron's ballistic transport in two-dimensional quantum waveguide. Journal of Applied Physics, v. 108, p. 053717-1-053717-6, 2010.

48 KORN, T. Time-resolved studies of electron and hole spin dynamics in modulation-doped $\mathrm{GaAs} / \mathrm{AlGaAs}$ quantum wells. Physics Reports-review section of physics letters, v. 494, n. 5, p. $415-445,2010$. 
49 KOGA, T.; NITTA, J.; VAN VEENHUIZEN, M. Ballistic spin interferometer using the rashba effect. Physical Review B, v. 70, n. 16, p. 161302-1-161302-4, 2004.

50 KOGA, T.; SEKINE, Y.; NITTA, J. Experimental realization of a ballistic spin interferometer based on the rashba effect using a nanolithographically defined square loop array. Physical Review B, v. 74, n. 4, p. 041302-1-041302-4, 2006.

51 KOGA, T.; NITTA, J.; TAKAYANAGI, H.; DATTA, S. Spin-filter device based on the rashba effect using a nonmagnetic resonant tunneling diode. Physical Review Letters, Woodbury, v. 88, n. 12, p. 126601-1-126601-4, 2002.

52 WOLF, S. A.; AWSCHALOM, D. D.; BUHRMAN, R. A.; DAUGHTON, J. M.; VON MOLNáR, S.; ROUKES, M. L.; CHTCHELKANOVA, A. Y.; TREGER, D. M. Spintronics: a spin-based electronics vision for the future. Science, Washington, v. 294, n. 5546, p. 1488-1495, 2001.

53 PARKIN, S. S. P.; HAYASHI, M.; THOMAS, L. Magnetic domain-wall racetrack memory. Science, Washington, v. 320, p. 190-194, 2008.

54 YUAN, Z.; CHEN, Z.; CUI, D.; MA, J.; HU, Q.; ZHOU, J.; ZHOU, Y. Normal incidence photoresponse in GaAs/AIGaAs quantum well infrared photodetector. Applied Physics Letters, Woodbury, v. 67, n. 7, p. 930-931, 1995.

55 LI, H. S.; KARUNASIRI, R. P. G.; CHEN, Y. W.; WANG, K. L. Electron intersubband normal incidence absorption in InGaAs/GaAs quantum wells. In: MOLECULAR-BEAM EPITAXY WORKSHOP, 6., 1993, Ottawa. Resumos... Chico-CA: AVS, c1993. p. 922-925.

56 ROSENCHER, E.; VINTER, B.; LEVINE, B. F. Intersubband transitions in quantum wells. New York: Plenum, 1992.

57 WEST, L. C.; EGLASH, S. J. First observation of an extremely large-dipole infrared transition within the conduction band of a GaAs quantum well. Applied Physics Letters, Woodbury, v. 46, n. 12, p. 1156-1158, 1985.

58 BERGER, V.; DUPONT, E.; DELACOURT, D.; VINTER, B.; VODJDANI, N.; PAPUCHON, M. Triple quantum well electron transfer infrared modulator. Applied Physics Letters, Woodbury, v. 61, n. 17, p. 2072-2074, 1992.

59 AS, D. J. Recent developments on non-polar cubic group III-nitrides for optoelectronic applications. In: CONFERENCE ON QUANTUM SENSING AND NANOPHOTONIC DEVICES, 7., 2010, Bellingham. Resumos... Los Angeles: SPIE, c2010. p. 76080G.

60 NAGASE, M.; AKIMOTO, R.; SIMOYAMA, T.; GUANGWEI, C.; MOZUME, T.; HASAMA, T.; ISHIKAWA, H. Enhancement of all-optical cross phase modulation in InGaAs/A- 
IAsSb coupled quantum wells using InAIAs coupling barriers. IEEE of Photonics Technology Letters, v. 20, n. 24, p. $2183-2185,2008$.

61 VOGEL, M.; VAGOV, A.; AXT, V. M.; SEILMEIER, A.; KUHN, T. Spin-sensitive intersubband dynamics of optically generated carriers in semiconductor quantum wells. Physical Review B, v. 80, n. 15, p. 155310-1-155310-7, 2009.

62 ALTMAN, S. L. Band theory of solids: an introduction from the point of view of symmetry. Oxford: Clarendon Press, 1991.

63 SANDS, D. E. Crystal systems and geometry, introduction to crystallography. Mineola, New York: Dover Publications, 1993.

64 KITTEL, C. Introduction to solid state physics. New York: Wiley, 1986.

65 YU, P.; CARDONA, M. Fundamentals of semiconductors: physics and materials properties. 3rd ed. New York: Springer, 2005.

66 HARRISON, W. O. Electronic structure and the properties of solids. San Francisco: Freeman, 1980.

67 LASSNIG, R. $\vec{k} \cdot \vec{p}$ theory, effective-mass approach, and spin splitting for two-dimensional electrons in GaAs-GaAlAs heterostructures. Physical Review B, v. 31, n. 12, p. 8076-8086, 1985.

68 ENDERLEIN, R.; HORING, N. M. Fundamentals of semiconductors and devices. New Jersey: World Scientific, 1997.

69 KANE, E. O. Band structure of indium antimonide. Journal of Physics and Chemistry of Solids, v. 1, n. 4, p. 249-261, 1957.

70 VURGAFTMAN, I.; MEYER, J. R.; RAM-MOHAN, L. R. Band parameters for III-V compound semiconductors and their alloys. Journal of Applied Physics, Woodbury, v. 89, n. 11 , p. 5815-5875, 2001.

71 IOFFE PHYSICO-TECHNICAL INSTITUTE. NSM archive - physical properties of semiconductors. Disponível em: <http://www.ioffe.ru/SVA/NSM//Semicond/>. Acesso em: Dezembro 2010.

72 DARNHOFER, T.; RÖSSLER, U. Effects of band structure and spin in quantum dots. Physical Review B, v. 47, n. 23, p. 16020-16023, 1993.

73 ZAWADZKI, W.; PFEFFER, P. Average forces in bound and resonant quantum states. Physical Review B, v. 64, n. 23, p. 235313-1-235313-5, 2001. 
74 ZAWADZKI, W.; PFEFFER, P. Spin splitting of subband energies due to inversion asymmetry in semiconductor heterostructures. Semiconductor Science and Technology, v. 19, n. 1, p. R1-R17, 2004.

75 HAIRER, E.; NØRSETT, S. P.; WANNER, G. Solving ordinary differential equations $i$ : nonstiff problems. New York: Springer-Verlag, 1993.

76 BERNARDES, E. O método numérico Numerov via computação algébrica. Disponível em: $<$ http://www.lia.if.sc.usp.br>. Acesso em: Janeiro 2009.

77 BERNARDES, E. A Numerov sixth order numerical scheme to accurately solve the 1d Poisson equation. In: INTERNATIONAL CONFERENCE ON PHYSICS AND APPLICATIONS OF SPIN-RELATED PHENOMENA IN SEMICONDUCTORS, 5., 2008, Foz do Iguaçu. Resumos... São Carlos: IFSC, c2008. p. 95.

78 BERNARDES, E. A direct Numerov sixth-order numerical scheme to accurately solve the unidimensional Poisson equation with Dirichlet boundary conditions Journal of Superconductivity and Novel Magnetism, v. 23, n. 1, p. 167-169, 2010.

79 MERZBACHER, E. Quantum mechanics. New York: John Wiley, 1970.

80 BOYKIN, T. B. Derivatives of the dirac delta function by explicit construction of sequences. American Journal of Physics, Woodbury, v. 71, n. 5, p. 462-468, 2003.

81 MOSQUEIRO, T. S.; BERNARDES, E.; EGUES, J. C. Spin-assisted optical transitions in zincblende quantum wells with two subbands. In: SÃO PAULO SCHOOL OF ADVANCED SCIENCE: SPINTRONICS AND QUANTUM COMPUTATION, 1., 2010, São Carlos. Resumos... São Carlos: IFSC, c2010. p. 21.

82 MOSQUEIRO, T. S.; BERNARDES, E.; EGUES, J. C. Full effective hamiltonians for one electron in the conduction subband of semiconducting heterostructues. In: LATIN AMERICAN SYMPOSIUM ON SOLID STATE PHYSICS, 20., 2011, Maragogi. Resumos... São Carlos: IFSC, c2011. p. 65.

83 ZHANG, Y. T.; SONG, Z. F.; LI, Y. C. Electron spin precession in two-dimensional electron gas with rashba spin-orbit coupling. Physics Letters A, Amsterdam, v. 373, n. 1, p. 144-147, 2008.

84 BARTOLO, B. Optical interactions in solids. New York: John Wiley et Sons, Inc, 1968.

85 BASTARD, G. Wave mechanics applied to semiconductor heterostructures. Les Ulis Cedex: Halsted Press, 1988.

86 MADELUNG, O. Introduction to solid-state theory. Berlin Heidelberg: Springer, 2008. 
87 WANGSNESS, R. Electromagnetic fields. 2nd ed. New York: Wiley, 2010.

88 WANG, Q.; STEDMAN, G. E. Spin-assisted matter-field coupling and lanthanide transition intensities. Journal of Physics B: atomic, molecular and optical physics, v. 26, n. 8, p. 1415, 1993.

89 GRIFFITHS, D. J. Introduction to electrodynamics. 3rd ed. Upper Saddle River: Benjamin Cummings, 1999.

90 SCHWABL, F. Advanced quantum mechanics. 4th ed. Berlin: Springer, 2008.

91 YANG, R. Q.; XU, J. M.; SWEENY, M. Selection rules of intersubband transitions in conduction-band quantum wells. Physical Review B, v. 50, n. 11, p. 7474-7482, 1994.

92 BURT, M. G. The evaluation of the matrix element for interband optical transitions in quantum wells using envelope functions. Journal of Physics: condensed matter, Bristol, v. 5, n. 24, p. 4091-4098, 1993.

93 MOSQUEIRO, T. S.; BERNARDES, E.; EGUES, J. C. Spin-assisted electron-photon couplings in zincblende quantum wells with two subbands. In: LATIN AMERICAN SYMPOSIUM ON SOLID STATE PHYSICS, 20., 2011, Maragogi. Resumos... São Carlos: IFSC, c2011. p. 64.

94 FEYNMAN, R. P.; LEIGHTON, R. B.; SANDS, M. The feynman lectures on physics. 2nd ed. Reading, Mass.: Addison-Wesley, 2005. (The Feynman Lectures on Physics, v. 3).

95 MOSQUEIRO, T. S.; BERNARDES, E.; EGUES, J. C. Transições ópticas em poços quânticos com interação spin-órbita. In: WORKSHOP DA PÓS GRADUAÇÃO DO IFSC, 13., 2009, São Carlos. Resumos... São Carlos: IFSC, c2009. p. 145.

96 WARBURTON, R. J.; GAUER, C.; WIXFORTH, A.; KOTTHAUS, J. P.; BRAR, B.; KROEMER, H. Intersubband resonances in InAs/AISb quantum wells: Selection rules, matrix elements, and the depolarization field. Physical Review B, v. 53, n. 12, p. 7903-7910, 1996. 


\section{APÊNDICE $A$}

\section{Cálculos auxiliares com matrizes $\mathbf{T}$}

Neste apêndice auxiliarei alguns cálculos da seção 2.7. Separei desta forma por acreditar que estas manipulações algébricas pudessem ofuscar um pouco a linha principal de raciocínio que segue o capítulo 2.

\section{A.1 Desenvolvimento de algumas expressões}

Desenvolverei algumas contas podem criar algum tipo de dúvida. Alguns poucos passos algébricos simples ficam a encargo do leitor. Defini os potenciais estruturais como $h_{j}(z)=$ $\delta_{j} h_{w}(z)$, em que $h_{w}(z)$ é um perfil de poço com altura unitária. As constantes $\delta_{j}$ são as alturas dos perfis de potencial (reveja a figura 2.4). Para semicondutores do tipo $I$, valem as desigualdades

$$
\begin{aligned}
& \delta_{6}>0 \\
& \delta_{8}<0 \\
& \delta_{7}<0
\end{aligned}
$$

Com a equação 2.5 .13 , é fácil ver que

$$
\boldsymbol{T} \cdot \boldsymbol{k} h_{8} \boldsymbol{T}^{\dagger} \cdot \boldsymbol{k}=\boldsymbol{T} \cdot \boldsymbol{k} g \boldsymbol{T}^{\dagger} \cdot \boldsymbol{k}=\delta_{8}\left[h_{w} k^{2}+\left(\boldsymbol{k} h_{w}\right) \cdot \boldsymbol{k}-\frac{i}{2} \boldsymbol{\sigma} \cdot\left(\boldsymbol{k} h_{w}\right) \times \boldsymbol{k}\right] .
$$

Similarmente,

$$
\boldsymbol{\sigma} \cdot \boldsymbol{k} h_{7} \boldsymbol{\sigma}^{\dagger} \cdot \boldsymbol{k}=\boldsymbol{T} \cdot \boldsymbol{k} g \boldsymbol{T}^{\dagger} \cdot \boldsymbol{k}=\delta_{7}\left[h_{w} k^{2}+\left(\boldsymbol{k} h_{w}\right) \cdot \boldsymbol{k}+i \boldsymbol{\sigma} \cdot\left(\boldsymbol{k} h_{w}\right) \times \boldsymbol{k}\right] .
$$


Substituindo estas igualdades e complementando com os coeficientes,

$$
\begin{aligned}
\frac{2 \mathcal{P}^{2}}{3 E_{g}^{2}} \boldsymbol{T} \cdot \boldsymbol{k} h_{8} \boldsymbol{T}^{\dagger} \cdot \boldsymbol{k}+\frac{\mathcal{P}^{2}}{3\left(E_{g}+\Delta_{g}\right)^{2}} \boldsymbol{\sigma} \cdot \boldsymbol{k} h_{7} \boldsymbol{\sigma} \cdot \boldsymbol{k}= \\
\frac{\mathcal{P}^{2}}{3}\left[\gamma_{2}^{\prime} h_{w} k^{2}+\gamma_{2}^{\prime}\left(\boldsymbol{k} h_{w}\right) \cdot \boldsymbol{k}-i \eta_{e} \boldsymbol{\sigma} \cdot\left(\boldsymbol{k} h_{w}\right) \times \boldsymbol{k}\right],
\end{aligned}
$$

em que é possível ver que

$$
\begin{aligned}
& \gamma_{2}^{\prime}=\frac{2 \delta_{8}}{E_{g}^{2}}+\frac{\delta_{7}}{\left(E_{g}+\Delta_{g}\right)^{2}} \\
& \eta_{e}=\frac{\delta_{8}}{E_{g}^{2}}-\frac{\delta_{7}}{\left(E_{g}+\Delta_{g}\right)^{2}}
\end{aligned}
$$

Estas constantes têm papéis importantes na hamiltoniana efetiva final: $\eta_{e}$ é parte do acoplamento spin-órbita e $\gamma_{2}^{\prime}$ é uma das correções para a massa efetiva. Vale comentar que Calsaverini et al obtêm este mesmo coeficiente para o acoplamento spin-órbita em sua dissertação ${ }^{1}$, mas a massa efetiva não seja calculada até segunda ordem.

\section{A.2 Expressão para a massa efetiva}

Em segunda ordem, a correção para a massa efetiva pode ser escrita como $\gamma_{2}^{\prime}+\gamma_{2} \delta_{6}$. Analisando a figura 2.4 , o leitor pode constatar

$$
\begin{aligned}
\delta_{8} & =E_{w}-E_{g}-\delta_{6}, \\
\delta_{7} & =\left(E_{w}+\Delta_{w}\right)-\left(E_{g}+\Delta_{g}\right)-\delta_{6} .
\end{aligned}
$$

Usando essas definições, posso expressar esta correção em uma forma mais direta:

$$
\gamma_{2}^{\prime}+\gamma_{2} \delta_{6}=2 \frac{E_{w}-E_{g}}{E_{g}^{2}}+\frac{E_{w}-E_{g}+\Delta_{g}-\Delta_{w}}{\left(E_{g}+\Delta_{g}\right)^{2}} .
$$

Eliminamos assim a dependência em $\delta_{6}$. Note que $E_{w}-E_{g}$ é a diferença de gaps entre as estruturas (fundo do poço e espaçadores).

\section{A.3 Hermitianicidade de uma expansão de Taylor}

Como não encontrei nenhuma referência realmente acessível sobre o tema e este é um assunto simples de ser tratado, segue um pequeno texto sobre essa questão. Ela é importante para garantir que, durante as expansões, teremos sempre a certeza de obter operadores que representem grandezas mensuráveis. 
Suponha que $\operatorname{adj}(\mathcal{A}):=\mathcal{A}^{\dagger}$ seja uma função que calcula o adjunto de um operador qualquer segundo o produto escalar que usamos costumeiramente em quântica (integral com duas funções de onda), i.e.,

$$
\langle v \mid \mathcal{A} n\rangle=\langle\operatorname{adj}(\mathcal{A}) v \mid n\rangle=\left\langle\mathcal{A}^{\dagger} v \mid n\right\rangle
$$

Implicitamente, utilizei uma notação que clareia quem atua em quem:

$$
|\mathcal{A} n\rangle:=\mathcal{A}|n\rangle
$$

Suponha agora que $\mathcal{A}^{\prime}=\mathcal{A}+\mathcal{B}$. Então, temos

$$
\left\langle v \mid \mathcal{A}^{\prime} n\right\rangle=\langle v \mid(\mathcal{A}+\mathcal{B}) n\rangle
$$

Como $(\mathcal{A}+\mathcal{B})|n\rangle=\mathcal{A}|n\rangle+\mathcal{B}|n\rangle$, então

$$
\left\langle v \mid \mathcal{A}^{\prime} n\right\rangle=\langle v \mid \mathcal{A} n\rangle+\langle v \mid \mathcal{B} n\rangle=\left\langle\mathcal{A}^{\dagger} v \mid n\right\rangle+\left\langle\mathcal{B}^{\dagger} v \mid n\right\rangle=\left\langle\mathcal{A}^{\prime \dagger} v \mid n\right\rangle .
$$

Além disso, para qualquer real $\alpha$, vale

$$
\langle v \mid \alpha \mathcal{A} n\rangle=\alpha\langle v \mid \mathcal{A} n\rangle=\alpha\left\langle\mathcal{A}^{\dagger} v \mid n\right\rangle=\alpha\left\langle\mathcal{A}^{\dagger} v \mid n\right\rangle,
$$

ou, reescrevendo de uma forma mais explícita, $\operatorname{adj}(\alpha \mathcal{A})=\alpha \operatorname{adj}(\mathcal{A})$. Acabamos de mostrar que adj é linear, i.e.,

$$
\operatorname{adj}(\alpha \mathcal{A}+\beta \mathcal{B})=\alpha \operatorname{adj}(\mathcal{A})+\beta \operatorname{adj}(\mathcal{B}) \quad \forall \alpha, \beta \in \mathbb{R}
$$

Os operadores hermitianos são, na verdade, "pontos fixos" desta função, ou seja, um operador pode ser chamado de hermitiano se, e só se,

$$
\operatorname{adj}(\mathcal{A})=\mathcal{A}
$$

Se tivermos uma soma de operadores, a condição de hermitianicidade torna-se

$$
\operatorname{adj}(\mathcal{A}+\mathcal{B})=\mathcal{A}+\mathcal{B}
$$

Note que, se $\mathcal{A}+\mathcal{B}$ é hermitiano, não é necessário que $\mathcal{A}$ e $\mathcal{B}$ sejam hermitianos individualmente. Portanto, a hermitianicidade de uma soma não necessariamente significa que seus fatores também sejam hermitianos. Vale comentar que a volta é verdadeira: a soma de operadores hermitianos é, obviamente, hermitiana.

No entanto, existe um tipo especial de soma, que nos interessa muito, para a qual podemos 
mostrar que cada um de seus termos é hermitiano se a soma toda o for. Imagine um operador $V(\lambda)$, em que $\lambda$ é um parâmetro real. Podemos expandi-lo em Taylor*,

$$
V(\lambda)=\sum_{j=0}^{\infty} \lambda^{j} \frac{d^{j} V}{d \lambda^{j}}(0)
$$

A expansão de Taylor é um polinômio, e neste caso um polinômio em $\lambda$. Se $V(\lambda)$ for hermitiano para um conjunto de parâmetros $\lambda$, então

$$
\operatorname{adj}(V(\lambda))=V(\lambda)
$$

Usando a expansão A.3.9,

$$
\operatorname{adj}(V(\lambda))=\operatorname{adj}\left(\sum_{j=0}^{\infty} \lambda^{j} \frac{d^{j} V}{d \lambda^{j}}(0)\right)=\sum_{j=0}^{\infty} \lambda^{j} \operatorname{adj}\left(\frac{d^{j} V}{d \lambda^{j}}(0)\right)
$$

em que usei implicitamente a linearidade. Voltando à condição de hermitianicidade A.3.10, chegamos à equação

$$
\sum_{j=0}^{\infty} \lambda^{j} \operatorname{adj}\left(\frac{d^{j} V}{d \lambda^{j}}(0)\right)=\sum_{j=0}^{\infty} \lambda^{j} \frac{d^{j} V}{d \lambda^{j}}(0) .
$$

A solução é bem conhecida, uma vez que os termos $\lambda^{j}$ representam uma base no espaço dos polinômios, e vou expressá-la na forma

$$
\operatorname{adj}\left(\frac{d^{j} V}{d \lambda^{j}}(0)\right)=\frac{d^{j} V}{d \lambda^{j}}(0)
$$

Esta última equação nos diz que a cada termo da expansão (ou seja, a cada inteiro $j$ diferente), os operadores são hermitianos. Este resultado representa um tipo de consistência que esperamos observar em nossos cálculos e que pode assegurar que estamos trabalhando algebricamente da forma correta.

O assunto desta seção leva em conta a sesquilinearidade do produto interno que usamos em mecânica quântica. Se mudarmos o produto interno, estas conclusões podem não mais serem verdadeiras. O trabalho com operadores não hermitianos, ou com produtos internos diferentes, geram possibilidades ainda mais interessantes.

\footnotetext{
* Ou ao menos nos restringimos ao caso em que podemos expandi-lo em Taylor. Este representa a grande maioria dos casos de interesse.
} 


\section{APÊNDICE $B$}

\section{Expansão não relativística da equação de Dirac}

Gostaria de expor brevemente a aproximação não relativística da equação de Dirac. Este procedimento foi realizado pela primeira vez por Berestetskii e Landau*(1949 apud BERESTETSKII; PITAEVSKII; LIFSHITZ, 1986, p. $124^{22}$ ).

\section{B.1 Equação efetiva de partículas}

Meu intuito é traçar uma comparação com meu procedimento, ao qual dedica-se o capítulo 2. No entanto, para tornar a comparação ainda mais clara, vou tornar este apêndice um pouquinho mais interessante usando os potenciais para partículas e antipartículas diferentes (usando a nomenclatura de Winkler ${ }^{41}$ ). A equação de Dirac pode ser escrita na forma ${ }^{22,23,36}$

$$
\left(\begin{array}{cc}
\mathbb{1}_{2 \times 2}\left(V_{p}+m c^{2}\right) & c \boldsymbol{\sigma} \cdot \boldsymbol{P} \\
c \boldsymbol{\sigma} \cdot \boldsymbol{P} & \mathbb{1}_{2 \times 2}\left(V_{a}-m c^{2}\right)
\end{array}\right) \Psi=\epsilon \Psi
$$

em que $\Psi$ é um quadrivetor ${ }^{\dagger}$ e os potenciais $V_{p}$ e $V_{a}$ são os potenciais para partículas e antipartículas, em ordem, que comentamos anteriormente. Não temos notícias de casos em que ocorre tal desigualdade, por isso em geral vale $V_{p}=V_{a}$. Utilizaremos esta desigualdade apenas para fortalecer nossa comparação. Os vetores $\boldsymbol{\sigma}$ e $\boldsymbol{P}$ são, respectivamente, o operador de spin (matrizes de Pauli) e momento linear.

*BERESTETSKII, V. B.; LANDAU, L. D. O vzaimodeistvii mezhdu elektronom i pozitronom. Zhurnal Eksperimentalnoi i Teoreticheskoi Fiziki, v. 19, n. 8, p. 673-679, 1949.

†Vetor de quatro componentes, nada relacionado a espaço-tempo. Também podemos formular a equação para um bispinor, um tensor de rank $2^{22}$. 
Separarei $\Psi$ da seguinte maneira:

$$
\Psi=\left(\begin{array}{l}
\phi \\
\chi
\end{array}\right)
$$

em que $\phi$ e $\chi$ são spinores de duas componentes. Esta separação decorre naturalmente da separação entre os blocos $2 \times 2$ na equação B.1.1. Da equação B.1.1, substitui $\Psi$ pelo spinor de B.1.2 para obtermos equações acopladas para $\phi$ e $\chi$,

$$
\begin{gathered}
\chi=\frac{c}{\epsilon-V+2 m c^{2}} \boldsymbol{\sigma} \cdot \boldsymbol{P} \phi \\
c^{2} \boldsymbol{\sigma} \cdot \boldsymbol{P} \frac{1}{\epsilon-V+2 m c^{2}} \boldsymbol{\sigma} \cdot \boldsymbol{P} \phi=(\epsilon-V) \phi .
\end{gathered}
$$

A componente $\phi$, privilegiada quase naturalmente $\ddagger$, não só descreve $\chi$, como também tem uma equação desacoplada que a determina.

\section{B.2 Correção da normalização e equação efetiva para partículas}

Ainda há um vínculo entre $\phi$ e $\chi$ : a densidade de probabilidade, que pode ser escrita como $\Psi^{\dagger} \Psi=\phi^{\dagger} \phi+\chi^{\dagger} \chi$. Usando argumentos similares ${ }^{\S}$ aos da seção 2.7.1, é possível obter um spinor (duas componentes) $\psi_{S}$ tal que, para qualquer intervalo $\Omega \subset \mathbb{R}^{3}$,

$$
\int_{\Omega} d^{3} r \psi_{S}^{\dagger} \psi_{S}=\int_{\Omega} d^{3} r \Psi^{\dagger} \Psi
$$

Note que isso significa que $\Psi$ e $\psi_{S}$ fornecem a mesma distribuição de probabilidades, muito embora sejam "funções de onda" diferentes. Resolver a equação B.2.1 para $\psi_{S}$ em função de $\phi$ não é uma tarefa simples, mas podemos fazer isso expandindo a equação B.2.1 em série de potências de $1 / c$. Como resultado, temos

$$
\psi_{S}=\left(1+\frac{P^{2}}{8 m^{2} c^{2}}\right) \phi+\mathcal{O}\left(1 / c^{3}\right)=\mathcal{L} \phi+\mathcal{O}\left(1 / c^{3}\right)
$$

Como já conhecemos a equação para $\phi$, podemos inverter a relação B.2.2. Substituindo $\mathcal{L}^{-1} \psi_{S}$ na equação B.1.3 e expandindo os denominadores de energia até segunda ordem em

\footnotetext{
†Para obter as equações B.1.3 e B.1.3 escolhi escrever $\chi$ em função de $\phi$. No entanto, pode-se mostrar, $a$ posteriori, que apenas $\chi$ se anula na aproximação não relativística.

$\S$ Na verdade, a seção 2.7.1 inspirou-se dos cálculos de Berestetskii e Landau.
} 
$1 / c$, obtive

$$
\begin{aligned}
{\left[\frac{P^{2}}{2 m}-\frac{P^{4}}{8 m c^{2}}+\frac{\hbar}{4 m^{2} c^{2}} \boldsymbol{\sigma} \cdot \nabla V_{a} \times \boldsymbol{P}\right.} & +\frac{\hbar^{2}}{8 m^{2} c^{2}} \nabla^{2} V_{p} \\
& \left.+\frac{1}{4 m^{2} c^{2}} \boldsymbol{P}\left(V_{a}-V_{p}\right) \cdot \boldsymbol{P}\right] \psi_{S}=\left(\epsilon-V_{p}\right) \psi_{S} .
\end{aligned}
$$

Para cairmos na equação "usualmente" conhecida como aproximação não relativística da equação de Dirac, basta impor $V_{p}=V_{a}$. Note que o termo proporcional a $\boldsymbol{P}\left(V_{a}-V_{p}\right) \cdot \boldsymbol{P}$ anula-se. Os outros termos são correções amplamente estudadas em sistemas atômicos e cristalinos. O termo proporcional a $P^{4}$ é usualmente conhecido como correção da massa relativística, por ser uma espécie de correção da energia devido a variações relativísticas da massa. O termo proporcional a $\boldsymbol{\sigma} \cdot \nabla V \times \boldsymbol{P}$ é o famoso acoplamento spin-órbita, e que geralmente abre a degenerescência de spin. Por fim, o termo $\nabla^{2} V$ é conhecido como correção de Darwin, e introduz não-localidade à interação elétron-núcleo ${ }^{24}$.

Voltando à equação B.2.3, vemos que o termo de Darwin é uma contribuição exclusivamente oriunda do potencial de partículas. O acoplamento spin-órbita, por outro lado, é exclusivamente gerado pelas antipartículas. Por fim, o acoplamento $\boldsymbol{P}\left(V_{a}-V_{p}\right) \cdot \boldsymbol{P}$, que em geral não aparece por valer $V_{a}=V_{p}$, é híbrido: advém tanto das partículas, como das antipartículas. 


\section{APÊNDICE $C$}

\section{Comparação com Winkler}

Winkler apresenta a equação efetiva para a banda de condução como ${ }^{41}$

$$
\begin{aligned}
& H_{W}= \frac{\mathcal{P}^{2}}{3}\left[\frac{2}{E_{g}}+\frac{1}{E_{g}+\Delta_{g}}\right] k^{2}+V_{c}+ \\
& \frac{\mathcal{P}^{2}}{6}\left[\frac{2}{E_{g}^{2}}+\frac{1}{\left(E_{g}+\Delta_{g}\right)^{2}}\right] k^{2} V_{v}-\frac{\mathcal{P}^{2}}{3} i \boldsymbol{\sigma} \cdot\left[\frac{1}{E_{g}^{2}}-\frac{1}{\left(E_{g}+\Delta_{g}\right)^{2}}\right] \boldsymbol{k} V_{v} \times \boldsymbol{k}+ \\
& \frac{\mathcal{P}^{2}}{3}\left[\frac{2}{E_{g}^{2}}+\frac{1}{\left(E_{g}+\Delta_{g}\right)^{2}}\right]\left\{V_{c}-V_{v}, k^{2}\right\} .
\end{aligned}
$$

Ao transcrevermos a equação obtida por Winkler, anulamos o campo magnético. Os potenciais $V_{c}$ e $V_{v}$ representam, respectivamente, os potenciais da banda de condução $-E_{6}$ em nossos cálculos - e das bandas de valência $-E_{7}$ e $E_{8}$. Como os cálculos de Winkler valem apenas no caso particular em que $\delta_{7}=\delta_{8}$, vou me restringir a este caso específico, i.e., as alturas das barreiras formadas pelas bandas de valência são iguais.

Desenvolvendo o anticomutador da equação C.0.1,

$$
\left\{V_{c}-V_{v}, k^{2}\right\} f=2\left(V_{c}-V_{v}\right) k^{2} f+f k^{2}\left(V_{c}-V_{v}\right)+\boldsymbol{k}\left(V_{c}-V_{v}\right) \cdot \boldsymbol{k} f .
$$

Substituindo C.0.2 na C.0.1 obtemos

$$
\begin{gathered}
H_{W}=\frac{\mathcal{P}^{2}}{3}\left[\frac{2}{E_{g}}+\frac{1}{E_{g}+\Delta_{g}}+\left\{\frac{2}{E_{g}^{2}}+\frac{1}{\left(E_{g}+\Delta_{g}\right)^{2}}\right\}\left(V_{c}-V_{v}\right)\right] k^{2}+V_{c}+ \\
\frac{\mathcal{P}^{2}}{6}\left[\frac{2}{E_{g}^{2}}+\frac{1}{\left(E_{g}+\Delta_{g}\right)^{2}}\right] k^{2}\left(V_{v}-2 V_{c}\right)-\frac{\mathcal{P}^{2}}{3} i \boldsymbol{\sigma} \cdot\left[\frac{1}{E_{g}^{2}}-\frac{1}{\left(E_{g}+\Delta_{g}\right)^{2}}\right] \boldsymbol{k} V_{v} \times \boldsymbol{k}+ \\
\frac{\mathcal{P}^{2}}{3}\left[\frac{2}{E_{g}^{2}}+\frac{1}{\left(E_{g}+\Delta_{g}\right)^{2}}\right] \boldsymbol{k}\left(V_{c}-V_{v}\right) \cdot \boldsymbol{k}
\end{gathered}
$$

Podemos ver, comparando C.0.3 e 2.7.27, que estão presentes na equação C.0.3 a correção de segunda ordem da massa (dependente da posição!), o acoplamento spin-órbita, o acoplamento 
momento-campo elétrico e o termo de Darwin.

A única diferença está no termo de Darwin, que depende do potencial da banda de valência e da banda de condução. Segundo meus cálculos, o potencial de Darwin deve depender apenas do potencial da banda de condução. O único termo "híbrido", quanto à sua origem, é o acoplamento momento-campo elétrico. Segundo Winkler ${ }^{41}$, "If we assume that we have different potentials for the particles and antiparticles, [...] the Darwin term for the particles depend only on the potentials for the antiparticles". No entanto, no apêndice B eu mostro que a correção de Darwin depende do "potencial de partículas", e não de antipartículas como sugerido. Isso reforça nossa convicção de que o termo de Darwin, na equação efetiva para a banda de condução, deve depender apenas do potencial da banda de condução.

Notemos por fim que se o denominador do termo de Darwin, da equação C.0.1, fosse 3 em vez de 6 , o resultado acabaria coincidindo. Isso sugere, singelamente, uma possível forma de concordância entre os dois resultados. 


\section{APÊNDICE $D$}

\section{Detalhes sobre a diagonalização}

Neste apêndice, gostaria de comentar alguns detalhes sobre as soluções para a banda de condução. Sugiro examinarmos exatamente a mesma hamiltoniana que no capítulo 3, mas vendo o problema por outro ponto de vista que pode ajudar a esclarecer o procedimento utilizado na seção 3.6. Por simplicidade, vamos nos restringir ao caso simétrico, em que os gates não quebram a simetria do potencial estrutural e as funções de onda do poço sem spin-órbita têm paridade definida.

Para que o leitor não tenha que ficar procurando muitas equações em capítulos diversos, algumas das equações a seguir são copiadas de outros capítulos com suas respectivas numerações mantidas.

\section{D.1 Hamiltoniana efetiva e a base de estados}

No capítulo 2, deduzi a hamiltoniana 2.7.40 efetiva para a banda de condução na base $\{|\uparrow\rangle,|\downarrow\rangle\}$ (portanto, é uma matriz $2 \times 2$ ). No capítulo 3, reescrevi esta hamiltoniana numa forma mais apresentável na equação 3.1.1, a saber,

$$
H_{2}=\left[\frac{\hbar^{2}}{2 m} \nabla^{2}+h_{6}+V+\alpha_{D} \frac{d^{2}}{d z^{2}}\left(\delta_{6} h_{w}+V\right)+\alpha_{M} \frac{d h_{w}}{d z} \frac{d}{d z}\right] \mathbb{1}_{2 \times 2}+\eta(z) s_{y},
$$

em que defini

$$
s_{y}:=k_{x} \sigma_{y}-k_{y} \sigma_{x}=\left(\begin{array}{cc}
0 & -i k_{-} \\
i k_{+} & 0
\end{array}\right) .
$$

A única parte não diagonal é a matriz $s_{y}$, que pode ser diagonalizada pelos vetores

$$
| \pm\rangle=\frac{\mp i e^{-i \theta}|\uparrow\rangle+|\downarrow\rangle}{\sqrt{2}}
$$


associados aos autovalores $\pm k_{\|}$. Na base $\{|+\rangle,|-\rangle\}, s_{y}=k_{\| \prime} \sigma_{z}{ }^{*}$ e podemos reescrever 3.1.1 como

$$
H_{2}=H_{0} \mathbb{1}_{2 \times 2}+\eta(z) k_{\|} \sigma_{x}=\left(\begin{array}{cc}
H_{0}+\eta(z) k & 0 \\
0 & H_{0}-\eta(z) k
\end{array}\right),
$$

em que defino o operador diferencial (não atua em spin, mas apenas nas coordenadas $(x, y, z)$ espaciais)

$$
H_{0}:=\frac{\hbar^{2}}{2 m} \nabla^{2}+h_{6}+V-\alpha_{D} \frac{d^{2}}{d z^{2}}\left(\delta_{6} h_{w}+V\right)+\alpha_{s} \frac{d h_{w}}{d z} \frac{d}{d z}
$$

Suponha que existam duas funções $\phi_{-1}$ e $\phi_{1}$, reais e ortonormais ${ }^{\dagger}$ entre si, tais que

$$
H_{0} \phi_{j}(z)=\epsilon_{j} \phi_{j}(z)
$$

e, além disso,

$$
\phi_{j}(z)=j \phi_{j}(-z)
$$

ou seja, uma é par $(j=1)$ e outra é impar $(j=-1)$. Se $H_{0}$ só admitir dois estados ligados, ocorre que $\left\{\phi_{0}, \phi_{1}\right\}$ é uma base para as soluções de $H_{0}$. Para continuar, vou deixar explícita uma hipótese quase que natural, mas que pode esclarecer algumas possíveis dúvidas.

Hipótese 1 A base de funções de $H_{0} \mathbb{1}_{2 \times 2}$ é base de $H_{2}$.

Esta hipótese é razoável do ponto de vista físico. O potencial estrutural (que vem em ordem zero em $1 / E_{g}$ ) é o potencial confinador do elétron. A montagem da heteroestrutura determina a largura e profundidade deste poço. Portanto, a informação física mais forte, em algum sentido, está em $H_{0}$ e o acoplamento spin-órbita (ou qualquer outro termo menos intenso ou de ordens mais elevadas em $\left.1 / E_{g}\right)$ não deve alterar a "física do problema". Portanto, é razoável que os estados admitidos por $H_{0}$, com a adição de spin, formem base para os estados admitidos por $\mathrm{H}_{2}$.

Desta hipótese, segue que podemos construir uma base para $H_{2}$ a partir destas duas funções $\phi_{j}$. Por clareza, defini

$$
|+\rangle:=|+1\rangle=\left(\begin{array}{l}
1 \\
0
\end{array}\right) \quad \text { e } \quad|-\rangle:=|-1\rangle=\left(\begin{array}{l}
0 \\
1
\end{array}\right) .
$$

De fato, ocorre

$$
H_{0} \mathbb{1}_{2 \times 2} \phi_{j}(z)|\mu\rangle=H_{0} \phi_{j} \mathbb{1}_{2 \times 2}|\mu\rangle=\epsilon_{j} \phi_{j}(z)|\mu\rangle
$$

* Matriz de Pauli

$\dagger$ Via produto interno usual. 
e portanto $\left\{\phi_{1}|+\rangle, \phi_{-1}|+\rangle, \phi_{1}|-\rangle, \phi_{-1}|-\rangle\right\}$ é base para $H_{0} \mathbb{1}_{2 \times 2}$; ainda, da hipótese que propus, também é base para $\mathrm{H}_{2}$.

\section{D.2 Revisitando Álgebra Linear}

Decorre desta hipótese e da montagem de nossa base que $H_{2} \phi_{j}|\mu\rangle$ é por si só um vetor descrito por esta base, logo

$$
H_{2} \phi_{j}(z)|\mu\rangle=\sum_{\nu, p \in\{-1,1\}} \alpha_{j p}^{\mu \nu} \phi_{p}(z)|\nu\rangle .
$$

Os coeficientes $\alpha_{j p}^{\mu \nu}$ indicam a projeção do vetor $H_{2} \phi_{j}|\mu\rangle$ nos vetores $\phi_{p}|\nu\rangle$. Para obtê-los, usei a ortonormalidade

$$
\alpha_{j p}^{\mu \nu}=\left(\langle\nu| \phi_{p}\right)\left(H_{2} \phi_{j}|\mu\rangle\right)
$$

Como conhecemos tanto $H_{0}$ como $\eta(z)$, é possível calcular o produto escalar da equação D.2.2. De fato, estes valores são os elementos de matriz da hamiltoniana $4 \times 4$ tratada no capítulo 3.

Calcularei a seguir explicitamente estes coeficientes. Usando a equação D.1.2, temos

$$
H_{2} \phi_{j}(z)|\mu\rangle=\epsilon_{j} \phi_{j}(z)|\mu\rangle+\eta(z) \phi_{j}(z) \sigma_{x}|\mu\rangle \text {. }
$$

A parte matricial do termo envolvendo o acoplamento spin-órbita é bem simples,

$$
\sigma_{x}|\mu\rangle=\mu|\mu\rangle
$$

Portanto a parte de spin mantem-se diagonal e, analogamente à equação D.2.1,

$$
\eta(z) \phi_{j}(z) \sigma_{x}|\mu\rangle=\mu|\mu\rangle \sum_{p \in\{-1,1\}} \beta_{j p}^{\mu \mu} \phi_{p}(z)
$$

em que, analogamente à equação D.2.2,

$$
\beta_{j p}^{\mu \mu}=\left(\langle\mu| \phi_{p}\right)\left(\eta \phi_{j}|\mu\rangle\right)=\left(\phi_{p}, \eta \phi_{j}\right)
$$

Se o leitor voltar à seção 3.3, note que $\beta_{j p}^{\mu \mu}=\eta_{j p}$, com a diferença de que as etiquetas das funções de base mudaram de 0,1 para $1,-1$. Como nos restringimos ao caso simétrico, sabemos que $\eta_{11}=\eta_{-1-1}=0$ e que $\eta_{1,-1}$ não se anula por argumentos de paridade. Desta forma, terminamos com a igualdade

$$
\eta(z) \phi_{j}(z) \sigma_{x}|\mu\rangle=\mu \phi_{-j}(z)|\mu\rangle
$$


Note que agora sabemos como $H_{2}$ age sobre os vetores de base.

\section{D.3 Solução para a banda de condução}

Fixando $\mu$, então

$$
\begin{aligned}
H_{2} \phi_{1}(z)|\mu\rangle & =\left[\epsilon_{1} \phi_{j}(z)+\mu \phi_{-1}(z)\right]|\mu\rangle \\
H_{2} \phi_{-1}(z)|\mu\rangle & =\left[\epsilon_{-1} \phi_{j}(z)+\mu \phi_{1}(z)\right]|\mu\rangle
\end{aligned}
$$

As duas equações D.3.1 representam um sistema de equações a serem resolvidas simultaneamente. A solução é uma combinação $a \phi_{1}+b \phi_{-1}$ linear de $\phi_{1}$ e $\phi_{-1}$ tal que

$$
H_{2}\left[a \phi_{1}(z)+b \phi_{-1}(z)\right]|\mu\rangle=\epsilon\left[a \phi_{1}(z)+b \phi_{-1}(z)\right]|\mu\rangle
$$

Reescrevendo esta equação na forma matricial,

$$
\left(\begin{array}{cc}
\epsilon_{1} & \eta_{1,-1} k_{\|} \\
\eta_{1,-1} k_{\|} & \epsilon_{-1}
\end{array}\right)\left(\begin{array}{l}
a \\
b
\end{array}\right)=\epsilon\left(\begin{array}{l}
a \\
b
\end{array}\right)
$$

A solução deste sistema linear já conhecemos: é exatamente o que resolvi na seção 3.6, e que pode ser escrita, usando a notação deste apêndice, como

$$
\psi_{\lambda}|\mu\rangle=\left[\frac{\mu \lambda \eta_{1,-1} \lambda}{\Omega(k)+\lambda \epsilon_{-}} \phi_{1}(z)+\phi_{-1}(z)\right]|\mu\rangle,
$$

em que $\psi=a \phi_{1}+b \phi_{-1}$ é a solução desejada, $\lambda$ etiqueta as duas soluções de $\epsilon \mathrm{e}$

$$
\epsilon_{-}=\frac{\epsilon_{-1}-\epsilon_{1}}{2} .
$$

